\title{
Summations of large logarithms by parton showers
}

\author{
Zoltán Nagy ${ }^{*}$ \\ DESY, Notkestrasse 85, 22607 Hamburg, Germany \\ Davison E. Soper $\oplus^{\dagger}$ \\ Institute for Fundamental Science, University of Oregon, Eugene, Oregon 97403-5203, USA
}

(Received 13 November 2020; accepted 23 July 2021; published 29 September 2021)

\begin{abstract}
We propose a method to examine how a parton shower sums large logarithms. In this method, one works with an appropriate integral transform of the distribution for the observable of interest. Then, one reformulates the parton shower so as to obtain the transformed distribution as an exponential for which one can compute the terms in the perturbative expansion of the exponent. We apply this general program to the thrust distribution in electron-positron annihilation, using several shower algorithms. Of the approaches that we use, the most generally applicable is to compute some of the perturbative coefficients in the exponent by numerical integration and to test whether they are consistent with next-to-leading-log summation of the thrust logarithms.
\end{abstract}

DOI: 10.1103/PhysRevD.104.054049

\section{INTRODUCTION}

Parton shower event generators provide a way to approximately sum large logarithms in QCD. Consider an infrared safe observable labeled by $J$ in hadron-hadron, lepton-hadron, or lepton-lepton collisions at a large energy scale $\mu_{\mathrm{H}}$. Suppose that one is interested in a cross section $\hat{\sigma}_{J}(\boldsymbol{v})$ for the observable to take the value $\boldsymbol{v}$. The observable is characterized by a scale $\hat{Q}_{J}^{2}(\boldsymbol{v})$, such that the $\hat{\sigma}_{J}(\boldsymbol{v})$ is not sensitive to parton splittings at a scale smaller than $\hat{Q}_{J}^{2}(v)$. For instance, one might be interested in the $\boldsymbol{k}_{\perp}$ distribution in the Drell-Yan process in hadron-hadron collisions. Then $\boldsymbol{v}=\boldsymbol{k}_{\perp}$ and $\hat{Q}_{J}^{2}(\boldsymbol{v}) \sim \boldsymbol{k}_{\perp}^{2}$. If $\hat{Q}_{J}^{2}(\boldsymbol{v}) \sim \mu_{\mathrm{H}}^{2}$, one can use straightforward QCD perturbation theory to calculate $\hat{\sigma}_{J}(\boldsymbol{v})$. However, if $\hat{Q}_{J}^{2}(\boldsymbol{v}) \ll \mu_{\mathrm{H}}^{2}$, the perturbative expansion for $\hat{\sigma}_{J}(\boldsymbol{v})$ will contain large logarithms, $\log \left(\mu_{\mathrm{H}}^{2} / \hat{Q}_{J}^{2}(\boldsymbol{v})\right)$.

Often, one can analyze these logarithms by taking an appropriate integral transform of $\hat{\sigma}_{J}(\boldsymbol{v})$. Then one calculates a cross section $\sigma_{J}(\boldsymbol{r})$ depending on a variable or variables $\boldsymbol{r}$. The cross section $\sigma_{J}(\boldsymbol{r})$ contains logarithms $L(\boldsymbol{r})$ that are large when $\boldsymbol{r}$ approaches a limit. For instance, one might take the Fourier transform, with transverse position $\boldsymbol{b}$, of the Drell-Yan $\boldsymbol{k}_{\perp}$ distribution. In this example, $\boldsymbol{r}$ stands for $\boldsymbol{b}$,

\footnotetext{
ZZoltan.Nagy@desy.de

†soper@uoregon.edu
}

Published by the American Physical Society under the terms of the Creative Commons Attribution 4.0 International license. Further distribution of this work must maintain attribution to the author(s) and the published article's title, journal citation, and DOI. Funded by SCOAP ${ }^{3}$. the limit is $\boldsymbol{b}^{2} \rightarrow \infty$, and the logarithm is $L=\log \left(\boldsymbol{b}^{2} \mu_{\mathrm{H}}^{2}\right)$. Typically the cross section then has the form

$$
\sigma_{J}(\boldsymbol{r})=c_{0}\left\{1+\sum_{n=1}^{\infty} \sum_{j=0}^{2 n} c(n, j) \alpha_{\mathrm{s}}^{n}\left(\mu_{\mathrm{H}}^{2}\right) L^{j}(\boldsymbol{r})\right\}
$$

The logarithms $L^{j}(\boldsymbol{r})$ arise in QCD from the soft and collinear singularities of the theory. These same soft and collinear singularities are contained in the splitting functions of a parton shower algorithm. Thus running a parton shower event generator to calculate $\sigma_{J}(\boldsymbol{r})$ will produce an approximation to the series in Eq. (1). That is, the parton shower approximately sums the large logarithms. The object of this paper is to investigate the form of the result of this summation. ${ }^{1}$

To exhibit the summation of logarithms, we rearrange the parton shower algorithm so that it is specialized to calculate just $\sigma_{J}(\boldsymbol{r})$ and so that it expresses $\sigma_{J}(\boldsymbol{r})$ directly in terms of an exponential

$$
\mathbb{e} \exp \left(\int_{\mu_{f}^{2}}^{\mu_{\mathrm{H}}^{2}} \frac{d \mu^{2}}{\mu^{2}} \mathcal{S}_{\mathcal{Y}}\left(\mu^{2} ; \boldsymbol{r}\right)\right)
$$

where $\mathbb{T}$ indicates ordering in $\mu^{2}$. The integral of $\mathcal{S}_{\mathcal{Y}}\left(\mu^{2} ; \boldsymbol{r}\right)$ in the exponent has an expansion

\footnotetext{
${ }^{1}$ The analysis applies not just when $\sigma_{J}(\boldsymbol{r})$ represents an integral transform of some other distribution, but also whenever the operator $\mathcal{O}_{J}(\boldsymbol{r})$ that we use to measure $\sigma_{J}(\boldsymbol{r})$ after the shower has an inverse. That is, $\mathcal{O}_{J}(\boldsymbol{r})$ must have no eigenvalues equal to zero.
} 


$$
\int_{\mu_{f}^{2}}^{\mu_{\mathrm{H}}^{2}} \frac{d \mu^{2}}{\mu^{2}} \mathcal{S}_{\mathcal{Y}}\left(\mu^{2} ; \boldsymbol{r}\right)=\sum_{n=1}^{\infty} \alpha_{s}^{n}\left(\mu_{\mathrm{H}}^{2}\right) \sum_{j=0}^{2 n} e(n, j) L^{j}(\boldsymbol{r}) .
$$

The operator $\mathcal{S}_{\mathcal{Y}}\left(\mu^{2} ; \boldsymbol{r}\right)$ is determined by the parton splitting operator $\mathcal{S}\left(\mu^{2}\right)$ in the original shower. This gives one direct access to the coefficients $e(n, j)$. With this representation, one has the potential to prove that $e(n, j)=0$ for $j>n+1$. The terms with $j=n+1$ are called leading$\log$ (LL) terms and the terms with $j=n$ are called next-toleading-log (NLL) terms. One also has the potential to prove that $e(n, j)$ for $j=n+1$ and for $j=n$ are what is expected in full QCD if a full QCD result is known.

There is a long history of investigations of how well parton shower event generators reproduce the expectation from full QCD for the large logarithm expansion of Eq. (3). We can provide some examples. For instance, Marchesini and Webber argued that a parton shower event generator based on angular ordering would better sum large logarithms than alternative formulations [1]. Ref. [2] found that the event generator [1] of Marchesini and Webber, a precursor to HeRwIG [3,4], agreed with the analytic QCD summation of double logarithms [5] for the energy-energy correlation function in $e^{+} e^{-}$annihilation better than an alternative event generator due to Gottschalk [6]. In 1991, Catani, Webber, and Marchesini compared QCD theory and the structure of parton shower algorithms for several observables that involve large logarithms and concluded that a small adjustment in the parton shower algorithm could improve the summation of the large logarithms [7]. The connection between parton showers and large nonglobal logarithms was investigated in [8]. One paper [9] argued that a dipole parton shower might not properly sum the logarithms that are encoded in the Dokshitzer-Gribov-Lipatov-Altarelli-Parisi (DGLAP) evolution equation for the distribution of hadrons in a final state parton. However, two investigations $[10,11]$ showed that this argument was not correct. A similar investigation showed analytically that a virtuality ordered dipole shower correctly sums the double logs that appear in the Drell-Yan transverse momentum distribution [12]. Some issues in large logarithm summations in dipole parton showers were investigated in [13]. The logarithmic accuracy of final state parton showers was investigated at order $\alpha_{s}^{2}$ in [14,15]. A more powerful analysis for $e^{+} e^{-}$annihilation was undertaken in [16]. A very recent paper [17] addresses corrections to the leading color approximation in a dipole parton shower in order to maintain proper color factors for leading logarithm terms.

Our plan for Secs. II through X of this paper is to develop the general theory behind the representation (2) along the lines of Ref. [18]. In this exposition, we also present the main steps of the construction of Ref. [18] in a form that, in our opinion, makes these steps more transparent. Then, starting in Sec. XI, we apply the representation (2) to an important example, the thrust distribution in electronpositron annihilation. We consider just the thrust distribution and not other distributions involving large logarithms. However, we look in some detail at how the exact form of the shower algorithm affects the results.

Since the construction presented in this paper is simpler for $e^{+} e^{-}$annihilation than for hadron-hadron or electronhadron collisions and since Secs. XI through XXVIII are quite self-contained, some readers may prefer to jump to the later sections before reading the more general analysis in the earlier sections.

\section{PARTON SHOWER FROM PERTURBATION THEORY}

The starting point for the general analysis is the perturbative cross section for an infrared safe observable in hadron-hadron collisions. This starting point also applies with some simplifications also to electron-hadron and electron-positron collisions. We describe this structure briefly here. A more detailed explanation can be found in Ref. [18].

The parton shower is described as in our parton shower event generator DEDUCTOR [19-26] using operators on a vector space, the "statistical space," that describes the momenta, flavors, colors, and spins for all of the partons created in a shower as the shower develops. The colors and spins are quantum variables and are described using a density matrix. With $m$ final state partons plus two initial state partons with labels "a" and "b," the partons carry labels a, b, $1,2, \ldots, m$. The partons have momenta $\{p\}_{m}=$ $\left\{p_{\mathrm{a}}, p_{\mathrm{b}}, p_{1}, \ldots, p_{m}\right\}$ and flavors $\{f\}_{m}$. We take the partons to be massless: $p_{i}^{2}=0$. For color, there are ket color basis states $\left|\{c\}_{m}\right\rangle$ and bra color basis states $\left\langle\left\{c^{\prime}\right\}_{m}\right|$. We use the trace basis, as described in Ref. [19]. For spin, there are ket basis states $\left|\{s\}_{m}\right\rangle$ and bra basis states $\left\langle\left\{s^{\prime}\right\}_{m}\right|$. Then the $m$-parton basis states for the statistical space are denoted by $\left.\mid\left\{p, f, c, c^{\prime}, s, s^{\prime}\right\}_{m}\right)$. A vector $\left.\mid \rho\right)$ in the statistical space is a linear combination of the basis states. The statistical space is introduced in some detail in Secs. 2 and 3 of Ref. [19]. These sections also show how shower evolution is expressed using evolution operators that act on the statistical space. (However, in the present paper the names of the operators follow Ref. [18] rather than Ref. [19].) The spin basis is described in Sec. 5 of Ref. [19] and the color basis is described in Sec. 7 of Ref. [19].

In Secs. II through X of this paper, we maintain a general framework with full color and spin. Practical parton shower programs $[4,27,28]$ typically average over spins, so that no spin quantum numbers appear in the shower equations. One can then carry out the analysis of the summation of large logarithms using the spin averaged shower, as we do starting in Sec. XI. For color, parton shower programs often use the leading color (LC) approximation, which provides the leading term in an expansion in powers of $1 / N_{c}^{2}=1 / 9$. With this approach, the color states $\left\{c, c^{\prime}\right\}_{m}$ do not affect 
the splitting probabilities, which are simply proportional to a factor $C_{\mathrm{F}}$ or $C_{\mathrm{A}}$. Our program, DEDUCTOR, uses what is called the LC+ approximation [22]. Thus we mostly use full color in this paper with the understanding that one could approximate to the LC+ or LC level if desired. We discuss this further and relate the discussion to Ref. [17] in Sec. XXV.

\section{A. Perturbative cross section}

If the QCD matrix element is calculated up to a given order, $\alpha_{s}^{K}$, the cross section is

$\sigma_{J}(\boldsymbol{r})=\left(1\left|\mathcal{O}_{J}(\boldsymbol{r}) \mathcal{F}_{0}\right| \rho\left(\mu_{\mathrm{R}}^{2}\right)\right)+\mathcal{O}\left(\alpha_{s}^{K+1}\right)+\mathcal{O}\left(\Lambda_{\mathrm{QCD}}^{2} / Q_{J}^{2}(\boldsymbol{r})\right)$.

Here the renormalized perturbative QCD density operator is represented by a vector in the statistical space $\left.\mid \rho\left(\mu_{\mathrm{R}}^{2}\right)\right)$. It is based on the exact matrix elements and contains all the possible partonic final states at order $K$. The density operator is already renormalized, typically in the modified minimal subtraction ( $\overline{\mathrm{MS}}$ ) scheme, thus it is independent of the renormalization scale, $\mu_{\mathrm{R}}^{2}$, up to the desired order

$$
\left.\mu_{\mathrm{R}}^{2} \frac{d}{d \mu_{\mathrm{R}}^{2}} \mid \rho\left(\mu_{\mathrm{R}}^{2}\right)\right)=\mathcal{O}\left(\alpha_{s}^{K+1}\right) .
$$

The next factor in Eq. (4) is the operator of the bare parton distribution functions (PDFs),

$$
\mathcal{F}_{0}=\left[\mathcal{F}_{\mathrm{R}}\left(\mu_{\mathrm{R}}^{2}\right) \circ \mathcal{K}\left(\mu_{\mathrm{R}}^{2}\right) \circ \mathcal{Z}_{F}\left(\mu_{\mathrm{R}}^{2}\right)\right]
$$

Here the circles, $a \circ b$, represent convolutions in the momentum fraction variables. The renormalized PDF operator for the hadron-hadron initial state is $\mathcal{F}_{\mathrm{R}}\left(\mu_{\mathrm{R}}^{2}\right)$. The corresponding $\overline{\mathrm{MS}}$ subtraction of initial state singularities is done by the $\mathcal{Z}_{F}\left(\mu_{\mathrm{R}}^{2}\right)$ operator, which contains factors $1 / \epsilon^{n}$ in dimensional regularization. As described in Ref. [18], one should typically use something other than the $\overline{\mathrm{MS}}$ scheme to define the parton distribution functions used internally in the shower. The factor $\mathcal{K}\left(\mu_{\mathrm{R}}^{2}\right)$ transforms to the shower scheme for the parton distribution functions $\mathcal{F}_{\mathrm{R}}\left(\mu_{\mathrm{R}}^{2}\right)$. The bare PDF is scale independent,

$$
\mu_{\mathrm{R}}^{2} \frac{d}{d \mu_{\mathrm{R}}^{2}}\left[\mathcal{F}_{\mathrm{R}}\left(\mu_{\mathrm{R}}^{2}\right) \circ \mathcal{K}\left(\mu_{\mathrm{R}}^{2}\right) \circ \mathcal{Z}_{F}\left(\mu_{\mathrm{R}}^{2}\right)\right]=\mathcal{O}\left(\alpha_{s}^{K+1}\right) .
$$

This equation leads to the proper evolution equation of the renormalized PDFs.

The next factor in Eq. (4) is the operator $\mathcal{O}_{J}(\boldsymbol{r})$ representing an infrared (IR) safe measurement, characterized by a set of parameters $\boldsymbol{r}$.

After applying these operators, we have a sum and integral over basis states $\left.\mid\left\{p, f, c, c^{\prime}, s, s^{\prime}\right\}_{m}\right)$. Finally, we multiply by the statistical bra vector (1) and obtain a cross section after performing the integrations using

$$
\left(1 \mid\left\{p, f, c, c^{\prime}, s, s^{\prime}\right\}_{m}\right)=\left\langle\left\{c^{\prime}\right\}_{m} \mid\{c\}_{m}\right\rangle\left\langle\left\{s^{\prime}\right\}_{m} \mid\{s\}_{m}\right\rangle .
$$

(The spin states $\left|\{s\}_{m}\right\rangle$ are orthogonal and normalized, but the color states $\left|\{c\}_{m}\right\rangle$ in the trace basis that we use are not orthogonal and some of them are not normalized exactly to 1 [19]. The statistical bra vector $(1 \mid$ is defined in Sec. 3.5 of Ref. [19].)

If the calculation includes perturbative contributions up to $\alpha_{s}^{K}$, then there is an error term $\mathcal{O}\left(\alpha_{s}^{K+1}\right)$ in Eq. (4). The formula is based on standard QCD factorization for infrared safe observables. This has power suppressed corrections of order $\left[\Lambda_{\mathrm{QCD}}^{2} / Q_{J}^{2}(\boldsymbol{r})\right]^{p}$ where $Q_{J}^{2}(\boldsymbol{r})$ is the lowest scale that the measurement operator $\mathcal{O}_{J}(\boldsymbol{r})$ can resolve and $p>0$. In the rest of this paper, we mostly omit explicit mention of these error terms.

The expression in Eq. (4) simplifies substantially in electron-positron annihilation. In this case, we can replace the operator $\mathcal{F}_{0}$ by 1 .

We point out that Eq. (4) is valid only in $d=4-2 \epsilon$ dimensions. It is not directly useful for practical calculations.

\section{B. IR singular operator}

To define a good subtraction scheme for a fixed order calculation one can use the IR singular operator $\mathcal{D}\left(\mu_{\mathrm{R}}^{2}, \mu_{\mathrm{S}}^{2}\right)$ [18]. This operator has a perturbative expansion

$$
\mathcal{D}\left(\mu_{\mathrm{R}}^{2}, \mu_{\mathrm{S}}^{2}\right)=1+\sum_{n \geq 1}\left[\frac{\alpha_{s}\left(\mu_{\mathrm{R}}^{2}\right)}{2 \pi}\right]^{n} \mathcal{D}^{(n)}\left(\mu_{\mathrm{R}}^{2}, \mu_{\mathrm{S}}^{2}\right) .
$$

The operators $\mathcal{D}^{(n)}\left(\mu_{\mathrm{R}}^{2}, \mu_{\mathrm{S}}^{2}\right)$ are key to defining a parton shower algorithm in a general framework. For a first order shower, one uses only $\mathcal{D}^{(1)}\left(\mu_{\mathrm{R}}^{2}, \mu_{\mathrm{S}}^{2}\right)$, but in a general framework we consider $\mathcal{D}^{(n)}\left(\mu_{\mathrm{R}}^{2}, \mu_{\mathrm{S}}^{2}\right)$ for any $n$. This operator describes the IR singularity structure of partonic states $\left.\mid \rho\left(\mu_{\mathrm{R}}^{2}\right)\right)$. When $\mathcal{D}^{(n)}\left(\mu_{\mathrm{R}}^{2}, \mu_{\mathrm{S}}^{2}\right)$ acts on a state $\mid\{p, f, c$, $\left.\left.c^{\prime}, s, s^{\prime}\right\}_{m}\right)$ it produces new states $\left.\mid\left\{\hat{p}, \hat{f}, \hat{c}, \hat{c}^{\prime}, \hat{s}, \hat{s}^{\prime}\right\}_{\hat{m}}\right)$ with $m \leq \hat{m} \leq m+n$ such that the IR singularities of

$$
\left(\left\{\hat{p}, \hat{f}, \hat{c}, \hat{c}^{\prime}, \hat{s}, \hat{s}^{\prime}\right\}_{\hat{m}}\left|\mathcal{D}^{(n)}\left(\mu_{\mathrm{R}}^{2}, \mu_{\mathrm{S}}^{2}\right)\right|\left\{p, f, c, c^{\prime}, s, s^{\prime}\right\}_{m}\right)
$$

match the singularities of $n$th order QCD Feynman diagrams that connect these two states. Here the singularities include the factors $1 / \epsilon$ from virtual loop diagrams and they include the singular behavior of the diagrams when any two or more momenta $\hat{p}$ become collinear or some of the $\hat{p}$ become soft. A toy model with operators $\mathcal{D}^{(n)}\left(\mu_{\mathrm{R}}^{2}, \mu_{\mathrm{S}}^{2}\right)$ beyond $n=1$ is presented in Appendix A of Ref. [18].

The operator $\mathcal{D}^{(n)}\left(\mu_{\mathrm{R}}^{2}, \mu_{\mathrm{S}}^{2}\right)$ depends on two scales, the standard renormalization scale $\mu_{\mathrm{R}}^{2}$ and the shower scale $\mu_{\mathrm{S}}^{2}$. 
The shower scale acts as an ultraviolet (UV) cutoff that separates the IR and UV regions associated with $\mathcal{D}^{(n)}\left(\mu_{\mathrm{R}}^{2}, \mu_{\mathrm{S}}^{2}\right)$. All IR singularities are included, but only regions near these singularities with a scale, specified by a parameter $k^{2}$, satisfying $k^{2}<\mu_{\mathrm{s}}^{2}$ are included. There is, of course, some freedom in choosing how the UV cutoff is defined. Different prescriptions lead to differences in the shower ordering prescription in the parton shower algorithm produced by $\mathcal{D}^{(n)}\left(\mu_{\mathrm{R}}^{2}, \mu_{\mathrm{S}}^{2}\right)$.

The singular operator is based on the $\overline{\mathrm{MS}}$ renormalized matrix elements and is independent of the renormalization scale. Thus we have

$$
\mu_{\mathrm{R}}^{2} \frac{\partial}{\partial \mu_{\mathrm{R}}^{2}} \mathcal{D}\left(\mu_{\mathrm{R}}^{2}, \mu_{\mathrm{S}}^{2}\right)=\mathcal{O}\left(\alpha_{s}^{K+1}\right) .
$$

This allows us to choose the renormalization scale conveniently.

In order to avoid large logarithms of $\mu_{\mathrm{R}}^{2} / \mu_{\mathrm{S}}^{2}$, it is useful to relate the renormalization scale to the shower scale. We define

$$
\mu_{\mathrm{R}}^{2}=\kappa_{\mathrm{R}} \mu_{\mathrm{S}}^{2} .
$$

Then we can avoid large $\log \left(\kappa_{\mathrm{R}}\right)$ factors by choosing $\kappa_{\mathrm{R}}$ of order 1.

The singular operator is perturbative and we can always define its perturbative inverse operator,

$$
\mathcal{D}\left(\mu_{\mathrm{R}}^{2}, \mu_{\mathrm{S}}^{2}\right) \mathcal{D}^{-1}\left(\mu_{\mathrm{R}}^{2}, \mu_{\mathrm{S}}^{2}\right)=1,
$$

by working order by order in the perturbative expansion of Eq. (12).

\section{Fixed order cross section}

We can make Eq. (4) more useful by inserting 1 in the form $\mathcal{D} \mathcal{D}^{-1}$,

$\sigma_{J}(\boldsymbol{r})=\left(1\left|\mathcal{O}_{J}(\boldsymbol{r}) \mathcal{F}_{0} \mathcal{D}\left(\mu_{\mathrm{R}}^{2}, \mu_{\mathrm{S}}^{2}\right) \mathcal{D}^{-1}\left(\mu_{\mathrm{R}}^{2}, \mu_{\mathrm{S}}^{2}\right)\right| \rho\left(\mu_{\mathrm{R}}^{2}\right)\right)$.

We notice that the expression $\left.\mathcal{D}^{-1}\left(\mu_{\mathrm{R}}^{2}, \mu_{\mathrm{S}}^{2}\right) \mid \rho\left(\mu_{\mathrm{R}}^{2}\right)\right)$ is well defined in $d=4$ dimensions since the inverse of the singular operator removes all the IR singularities of $\left.\mid \rho\left(\mu_{\mathrm{R}}^{2}\right)\right)$. Accordingly, we define the subtracted hard matrix element by

$$
\left.\left.\mid \rho_{\mathrm{H}}\left(\mu_{\mathrm{R}}^{2}, \mu_{\mathrm{S}}^{2}\right)\right)=\lim _{\epsilon \rightarrow 0} \mathcal{D}^{-1}\left(\mu_{\mathrm{R}}^{2}, \mu_{\mathrm{S}}^{2}\right) \mid \rho\left(\mu_{\mathrm{R}}^{2}\right)\right) .
$$

This gives us

$$
\sigma_{J}(\boldsymbol{r})=\left(1\left|\mathcal{O}_{J}(\boldsymbol{r}) \mathcal{F}_{0} \mathcal{D}\left(\mu_{\mathrm{R}}^{2}, \mu_{\mathrm{S}}^{2}\right)\right| \rho_{\mathrm{H}}\left(\mu_{\mathrm{R}}^{2}, \mu_{\mathrm{S}}^{2}\right)\right) .
$$

We will use Eq. (15) to explore parton showers. First, however, suppose that we are interested only in the fixed order cross section. Then we can choose the scale $\mu_{\mathrm{S}}^{2}$ small enough that the measurement operator $\mathcal{O}_{J}(\boldsymbol{r})$ does not resolve parton momentum scales of order $\mu_{\mathrm{S}}^{2}$. Then $\mathcal{O}_{J}(\boldsymbol{r})$ commutes with $\mathcal{F}_{0} \mathcal{D}\left(\mu_{\mathrm{R}}^{2}, \mu_{\mathrm{S}}^{2}\right)$, giving us

$$
\sigma_{J}(\boldsymbol{r})=\left(1\left|\mathcal{F}_{0} \mathcal{D}\left(\mu_{\mathrm{R}}^{2}, \mu_{\mathrm{S}}^{2}\right) \mathcal{O}_{J}(\boldsymbol{r})\right| \rho_{\mathrm{H}}\left(\mu_{\mathrm{R}}^{2}, \mu_{\mathrm{S}}^{2}\right)\right) .
$$

One can calculate $\left(1 \mid \mathcal{F}_{0} \mathcal{D}\left(\mu_{\mathrm{R}}^{2}, \mu_{\mathrm{S}}^{2}\right)\right.$ in $d=4-2 \epsilon$ dimensions. The operator $\mathcal{D}\left(\mu_{\mathrm{R}}^{2}, \mu_{\mathrm{S}}^{2}\right)$ creates singularities, but the initial state singularities are removed by the operator $\mathcal{Z}_{F}\left(\mu_{\mathrm{R}}^{2}\right)$ in $\mathcal{F}_{0}$ and the final state singularities cancel after we multiply by $(1 \mid$ and integrate over the parton variables. Thus we obtain a finite result in the $\epsilon \rightarrow 0$ limit.

\section{Operators $\mathcal{V}$ and $\mathcal{X}_{1}$}

The operators $\mathcal{D}\left(\mu_{\mathrm{R}}^{2}, \mu_{\mathrm{S}}^{2}\right)$ and $\mathcal{F}_{0}$ are defined only in $d=4-2 \epsilon$ dimensions and are singular as $\epsilon \rightarrow 0$ and as parton momenta become soft or collinear. However, we have noted that $\left(1 \mid \mathcal{F}_{0} \mathcal{D}\left(\mu_{\mathrm{R}}^{2}, \mu_{\mathrm{S}}^{2}\right)\right.$ is finite in $d=4$ dimensions. It will prove useful to introduce an operator, $\mathcal{V}\left(\mu_{\mathrm{R}}^{2}, \mu_{\mathrm{S}}^{2}\right)$, that is finite in four dimensions, does not change the number of partons, leaves the parton momenta and flavors $\{p, f\}_{m}$ unchanged, and satisfies

$$
\left(1 \mid \mathcal{V}\left(\mu_{\mathrm{R}}^{2}, \mu_{\mathrm{S}}^{2}\right)=\lim _{\epsilon \rightarrow 0}\left(1 \mid \mathcal{F}_{0} \mathcal{D}\left(\mu_{\mathrm{R}}^{2}, \mu_{\mathrm{S}}^{2}\right) \mathcal{F}_{\mathrm{R}}^{-1}\left(\mu_{\mathrm{R}}^{2}\right) .\right.\right.
$$

The operator $\mathcal{V}\left(\mu_{\mathrm{R}}^{2}, \mu_{\mathrm{S}}^{2}\right)$ leaves $\{p, f\}_{m}$ unchanged, but it can act nontrivially on the color and spin space. Eq. (17) does not fully define the color and spin content of $\mathcal{V}\left(\mu_{\mathrm{R}}^{2}, \mu_{\mathrm{S}}^{2}\right)$. We discuss the definition further in Sec. IV, but for now, we need only Eq. (17).

Using $\mathcal{V}\left(\mu_{\mathrm{R}}^{2}, \mu_{\mathrm{S}}^{2}\right)$ we define a singular operator $\mathcal{X}_{1}\left(\mu_{\mathrm{R}}^{2}, \mu_{\mathrm{S}}^{2}\right)$ as

$$
\mathcal{X}_{1}\left(\mu_{\mathrm{R}}^{2}, \mu_{\mathrm{S}}^{2}\right)=\mathcal{F}_{0} \mathcal{D}\left(\mu_{\mathrm{R}}^{2}, \mu_{\mathrm{S}}^{2}\right) \mathcal{F}_{\mathrm{R}}^{-1}\left(\mu_{\mathrm{R}}^{2}\right) \mathcal{V}^{-1}\left(\mu_{\mathrm{R}}^{2}, \mu_{\mathrm{S}}^{2}\right),
$$

so that

$$
\left(1 \mid \mathcal{X}_{1}\left(\mu_{\mathrm{R}}^{2}, \mu_{\mathrm{S}}^{2}\right)=(1 \mid .\right.
$$

The " 1 " subscript distinguishes the operator $\mathcal{X}_{1}$ from the operator $\mathcal{X}$ used in Ref. [18] and suggests the normalization condition (19).

With $\mathcal{V}$ and $\mathcal{X}_{1}$, the cross section in Eq. (15) can be written as

$$
\sigma_{J}(\boldsymbol{r})=\left(1\left|\mathcal{O}_{J}(\boldsymbol{r}) \mathcal{X}_{1}\left(\mu_{\mathrm{R}}^{2}, \mu_{\mathrm{S}}^{2}\right) \mathcal{V}\left(\mu_{\mathrm{R}}^{2}, \mu_{\mathrm{S}}^{2}\right) \mathcal{F}_{\mathrm{R}}\left(\mu_{\mathrm{R}}^{2}\right)\right| \rho_{\mathrm{H}}\left(\mu_{\mathrm{R}}^{2}, \mu_{\mathrm{S}}^{2}\right)\right) .
$$

This form will be useful to help us define a parton shower.

Before we continue with the discussion of the parton shower cross section we introduce a more compact notation for operators with renormalization scale dependence. 
According to Eq. (11) the renormalization scale is always related to the shower scale; thus we can define

$$
\begin{aligned}
\mathcal{D}\left(\mu^{2}\right) & \equiv \mathcal{D}\left(\kappa_{\mathrm{R}} \mu^{2}, \mu^{2}\right), \\
\mathcal{X}_{1}\left(\mu^{2}\right) & \equiv \mathcal{X}_{1}\left(\kappa_{\mathrm{R}} \mu^{2}, \mu^{2}\right), \\
\mathcal{V}\left(\mu^{2}\right) & \equiv \mathcal{V}\left(\kappa_{\mathrm{R}} \mu^{2}, \mu^{2}\right), \\
\left.\mid \rho_{\mathrm{H}}\left(\mu^{2}\right)\right) & \left.\equiv \mid \rho_{\mathrm{H}}\left(\kappa_{\mathrm{R}} \mu^{2}, \mu^{2}\right)\right) .
\end{aligned}
$$

The PDF operator depends only on the renormalization scale and in this case the convention is a little different,

$$
\mathcal{F}\left(\mu^{2}\right)=\mathcal{F}_{\mathrm{R}}\left(\kappa_{\mathrm{R}} \mu^{2}\right) .
$$

The functions specified above then depend on $\kappa_{\mathrm{R}}$, but we do not display this dependence. With this more compact notation, Eq. (20) is written as

$$
\sigma_{J}(\boldsymbol{r})=\left(1\left|\mathcal{O}_{J}(\boldsymbol{r}) \mathcal{X}_{1}\left(\mu^{2}\right) \mathcal{V}\left(\mu^{2}\right) \mathcal{F}\left(\mu^{2}\right)\right| \rho_{\mathrm{H}}\left(\mu^{2}\right)\right) .
$$

\section{E. Operator $\mathcal{U}$ and parton shower}

The formula for the cross section $\sigma_{J}$ given in Eq. (23) is of limited usefulness if the scale $Q_{J}^{2}(\boldsymbol{r})$, representing the lowest scale that the measurement operator $\mathcal{O}_{J}(\boldsymbol{r})$ can resolve, is much smaller than the scale $\mu_{\mathrm{H}}^{2}$ of the hardest momentum transfer in $\left.\mid \rho_{\mathrm{H}}\left(\mu^{2}\right)\right)$. When that happens, $\sigma_{J}$ will contain logarithms $\log \left(\mu_{\mathrm{H}}^{2} / Q_{J}^{2}(\boldsymbol{r})\right)$ that need to be summed by looking for the most important terms at all orders of perturbation theory. To that end, one can use a parton shower algorithm.

To provide a parton shower, first set the scale $\mu^{2}$ in Eq. (23) to $\mu_{\mathrm{H}}^{2}$. Then define a scale $\mu_{f}^{2}$ that is certainly smaller than $Q_{J}^{2}(\boldsymbol{r})$. Typically, one chooses $\mu_{f}^{2}$ on the order of $1 \mathrm{GeV}^{2}$. Finally, insert $\left.1=\mathcal{X}_{1}\left(\mu_{f}^{2}\right) \mathcal{X}_{1}^{-1} \mu_{f}^{2}\right)$ into Eq. (23), giving

$$
\begin{aligned}
\sigma_{J}(\boldsymbol{r})= & \left(1 \mid \mathcal{O}_{J}(\boldsymbol{r}) \mathcal{X}_{1}\left(\mu_{f}^{2}\right) \mathcal{X}_{1}^{-1}\left(\mu_{f}^{2}\right) \mathcal{X}_{1}\left(\mu_{\mathrm{H}}^{2}\right)\right. \\
& \left.\times \mathcal{V}\left(\mu_{\mathrm{H}}^{2}\right) \mathcal{F}\left(\mu_{\mathrm{H}}^{2}\right) \mid \rho_{\mathrm{H}}\left(\mu_{\mathrm{H}}^{2}\right)\right) .
\end{aligned}
$$

Since $\mu_{f}^{2}<Q_{J}^{2}(\boldsymbol{r})$, the operator $\mathcal{O}_{J}(\boldsymbol{r})$ does not resolve partons at the scale $\mu_{f}^{2}$. Thus $\mathcal{O}_{J}(\boldsymbol{r})$ commutes with $\mathcal{X}_{1}\left(\mu_{f}^{2}\right)$, giving us

$$
\begin{aligned}
\sigma_{J}(\boldsymbol{r})= & \left(1 \mid \mathcal{X}_{1}\left(\mu_{f}^{2}\right) \mathcal{O}_{J}(\boldsymbol{r}) \mathcal{X}_{1}^{-1}\left(\mu_{f}^{2}\right) \mathcal{X}_{1}\left(\mu_{\mathrm{H}}^{2}\right)\right. \\
& \left.\times \mathcal{V}\left(\mu_{\mathrm{H}}^{2}\right) \mathcal{F}\left(\mu_{\mathrm{H}}^{2}\right) \mid \rho_{\mathrm{H}}\left(\mu_{\mathrm{H}}^{2}\right)\right)
\end{aligned}
$$

With the use of Eq. (19), this is

$$
\sigma_{J}(\boldsymbol{r})=\left(1\left|\mathcal{O}_{J}(\boldsymbol{r}) \mathcal{X}_{1}^{-1}\left(\mu_{f}^{2}\right) \mathcal{X}_{1}\left(\mu_{\mathrm{H}}^{2}\right) \mathcal{V}\left(\mu_{\mathrm{H}}^{2}\right) \mathcal{F}\left(\mu_{\mathrm{H}}^{2}\right)\right| \rho_{\mathrm{H}}\left(\mu_{\mathrm{H}}^{2}\right)\right) .
$$

The operator $\mathcal{X}_{1}^{-1}\left(\mu_{f}^{2}\right) \mathcal{X}_{1}\left(\mu_{\mathrm{H}}^{2}\right)$ is of special importance. We give it the name

$$
\mathcal{U}\left(\mu_{f}^{2}, \mu_{\mathrm{H}}^{2}\right)=\lim _{\epsilon \rightarrow 0} \mathcal{X}_{1}^{-1}\left(\mu_{f}^{2}\right) \mathcal{X}_{1}\left(\mu_{\mathrm{H}}^{2}\right) .
$$

This is the shower operator. It generates a parton shower starting at the scale $\mu_{\mathrm{H}}^{2}$ and ending at the scale $\mu_{f}^{2}$. Because of Eq. (19), the shower operator is probability preserving

$$
\left(1 \mid \mathcal{U}\left(\mu_{\mathrm{f}}^{2}, \mu_{\mathrm{H}}^{2}\right)=(1 \mid .\right.
$$

Using the notation $\mathcal{U}\left(\mu_{f}^{2}, \mu_{\mathrm{H}}^{2}\right)$, the cross section is

$$
\sigma_{J}(\boldsymbol{r})=\left(1\left|\mathcal{O}_{J}(\boldsymbol{r}) \mathcal{U}\left(\mu_{f}^{2}, \mu_{\mathrm{H}}^{2}\right) \mathcal{V}\left(\mu_{\mathrm{H}}^{2}\right) \mathcal{F}\left(\mu_{\mathrm{H}}^{2}\right)\right| \rho_{\mathrm{H}}\left(\mu_{\mathrm{H}}^{2}\right)\right) .
$$

We have perturbatively calculated matrix elements with their IR divergences subtracted in $\left.\mid \rho_{\mathrm{H}}\left(\mu_{\mathrm{H}}^{2}\right)\right)$. Then the operator $\mathcal{F}\left(\mu_{\mathrm{H}}^{2}\right)$ supplies parton distribution functions. The factor $\mathcal{V}\left(\mu_{\mathrm{H}}^{2}\right)$ serves to sum threshold logarithms $[18,26]$. An approximation to this factor is contained in DEDUCTOR although it is lacking in other current parton shower event generators. Next, the operator $\mathcal{U}\left(\mu_{f}^{2}, \mu_{\mathrm{H}}^{2}\right)$ generates the parton shower and the operator $\mathcal{O}_{J}(\boldsymbol{r})$ measures the desired observable in the multiparton state created by the shower. Finally, we multiply by (1| and integrate to get the desired cross section. We discuss $\mathcal{U}\left(\mu_{\mathrm{f}}^{2}, \mu_{\mathrm{H}}^{2}\right)$ and $\mathcal{V}\left(\mu_{\mathrm{H}}^{2}\right)$ in more detail in Secs. V and VI.

\section{OBSERVABLE DEPENDENT SHOWER EVOLUTION}

The operator $\mathcal{O}_{J}(\boldsymbol{r})$ in Eq. (29) could represent any infrared safe observable. In this paper, we have a particular sort of operator in mind. Consider, for example, the transverse momentum distribution of a $\mathrm{Z}$ boson produced in the Drell-Yan process. The operator that measures the transverse momentum $\boldsymbol{k}_{\perp}$ of the $\mathrm{Z}$ boson is defined as

$$
\begin{aligned}
& \left.\hat{\mathcal{O}}_{\mathrm{Z}}\left(\boldsymbol{k}_{\perp}\right) \mid\left\{p, f, c, c^{\prime}, s, s^{\prime}\right\}_{m}\right) \\
& \left.\quad=(2 \pi)^{2} \delta^{(2)}\left(\boldsymbol{k}_{\perp}-\boldsymbol{k}_{\mathrm{Z}}\left(\{p\}_{m}\right)\right) \mid\left\{p, f, c, c^{\prime}, s, s^{\prime}\right\}_{m}\right),
\end{aligned}
$$

where $\boldsymbol{k}_{\mathrm{Z}}\left(\{p\}_{m}\right)$ is the transverse momentum of the observed $\mathrm{Z}$ boson. The standard method for summing logarithms of $\boldsymbol{k}_{\perp}^{2} / M_{\mathrm{Z}}^{2}$ is to start with the Fourier transform of the $\boldsymbol{k}_{\perp}$ distribution. To measure this with a parton shower event generator, we can use the measurement operator

$$
\begin{aligned}
\left.\mathcal{O}_{\mathrm{Z}}(\boldsymbol{b}) \mid\left\{p, f, c, c^{\prime}, s, s^{\prime}\right\}_{m}\right) \\
=\int \frac{d \boldsymbol{k}_{\perp}}{(2 \pi)^{2}} e^{\mathrm{i} \boldsymbol{b} \cdot \boldsymbol{k}_{\perp}(2 \pi)^{2} \delta^{(2)}\left(\boldsymbol{k}_{\perp}-\boldsymbol{k}_{\mathrm{Z}}\left(\{p\}_{m}\right)\right)} \\
\left.\quad \times \mid\left\{p, f, c, c^{\prime}, s, s^{\prime}\right\}_{m}\right) \\
\left.=e^{\mathrm{i} \boldsymbol{b} \cdot \boldsymbol{k}_{\mathrm{Z}}\left(\{p\}_{m}\right)} \mid\left\{p, f, c, c^{\prime}, s, s^{\prime}\right\}_{m}\right) .
\end{aligned}
$$


We let $\mathcal{O}_{\mathrm{Z}}(\boldsymbol{b})$ serve as an example of the observable $\mathcal{O}_{J}(\boldsymbol{r})$ that we consider in this paper. There are many other similar examples. We will need one property of the observable $\mathcal{O}_{J}(\boldsymbol{r})$ beyond infrared safety: we assume that the operator $\mathcal{O}_{J}(\boldsymbol{r})$ has an inverse $\mathcal{O}_{J}^{-1}(\boldsymbol{r})$.

To analyze the cross section $\sigma_{J}(\boldsymbol{r})$, we start with the representation (23) with $\mu^{2}=\mu_{\mathrm{H}}^{2}$,

$$
\sigma_{J}(\boldsymbol{r})=\left(1\left|\mathcal{O}_{J}(\boldsymbol{r}) \mathcal{X}_{1}\left(\mu_{\mathrm{H}}^{2}\right) \mathcal{V}\left(\mu_{\mathrm{H}}^{2}\right) \mathcal{F}\left(\mu_{\mathrm{H}}^{2}\right)\right| \rho_{\mathrm{H}}\left(\mu_{\mathrm{H}}^{2}\right)\right) .
$$

Define an operator $\mathcal{Y}\left(\mu^{2} ; \boldsymbol{r}\right)$ that is finite in $d=4$ dimensions, leaves the number of partons and their momenta and flavors unchanged, and is related to $\mathcal{X}_{1}$ by

$$
\left(1 \mid \mathcal{Y}\left(\mu^{2} ; \boldsymbol{r}\right)=\left(1 \mid \mathcal{O}_{J}(\boldsymbol{r}) \mathcal{X}_{1}\left(\mu^{2}\right) \mathcal{O}_{J}^{-1}(\boldsymbol{r}) .\right.\right.
$$

Then define a new version of $\mathcal{X}_{1}$ that depends on the measurement parameters $\boldsymbol{r}$ by

$$
\mathcal{X}_{1}\left(\mu^{2} ; \boldsymbol{r}\right)=\mathcal{O}_{J}(\boldsymbol{r}) \mathcal{X}_{1}\left(\mu^{2}\right) \mathcal{O}_{J}^{-1}(\boldsymbol{r}) \mathcal{Y}^{-1}\left(\mu^{2} ; \boldsymbol{r}\right) .
$$

This gives us

$$
\left(1 \mid \mathcal{X}_{1}\left(\mu^{2}, \boldsymbol{r}\right)=(1 \mid\right.
$$

and

$$
\mathcal{O}_{J}(\boldsymbol{r}) \mathcal{X}_{1}\left(\mu^{2}\right)=\mathcal{X}_{1}\left(\mu^{2}, \boldsymbol{r}\right) \mathcal{Y}\left(\mu^{2} ; \boldsymbol{r}\right) \mathcal{O}_{J}(\boldsymbol{r})
$$

Then our cross section is

$\sigma_{J}(\boldsymbol{r})=\left(1\left|\mathcal{X}_{1}\left(\mu_{\mathrm{H}}^{2}, \boldsymbol{r}\right) \mathcal{Y}\left(\mu_{\mathrm{H}}^{2} ; \boldsymbol{r}\right) \mathcal{O}_{J}(\boldsymbol{r}) \mathcal{V}\left(\mu_{\mathrm{H}}^{2}\right) \mathcal{F}\left(\mu_{\mathrm{H}}^{2}\right)\right| \rho_{\mathrm{H}}\left(\mu_{\mathrm{H}}^{2}\right)\right)$.

With the use of Eq. (35), and commuting $\mathcal{O}_{J}(\boldsymbol{r})$ past $\mathcal{V}\left(\mu_{\mathrm{H}}^{2}\right)$ and $\mathcal{F}\left(\mu_{\mathrm{H}}^{2}\right)$, which do not change the partonic state, this becomes

$$
\sigma_{J}(\boldsymbol{r})=\left(1\left|\mathcal{Y}\left(\mu_{\mathrm{H}}^{2} ; \boldsymbol{r}\right) \mathcal{V}\left(\mu_{\mathrm{H}}^{2}\right) \mathcal{F}\left(\mu_{\mathrm{H}}^{2}\right) \mathcal{O}_{J}(\boldsymbol{r})\right| \rho_{\mathrm{H}}\left(\mu_{\mathrm{H}}^{2}\right)\right) .
$$

Here we measure $\mathcal{O}_{J}(\boldsymbol{r})$ at the hard state $\left.\mid \rho_{\mathrm{H}}\left(\mu_{\mathrm{H}}^{2}\right)\right)$, obtaining typically a very simple result. Then we measure $\mathcal{O}_{J}(\boldsymbol{r})$ inside the operator $\mathcal{Y}\left(\mu_{\mathrm{H}}^{2} ; \boldsymbol{r}\right)$. This operator has the potential to sum large logarithms.

We can also relate $\mathcal{Y}\left(\mu^{2} ; \boldsymbol{r}\right)$ to the shower operator $\mathcal{U}\left(\mu_{f}^{2}, \mu^{2}\right)$ with a small final scale $\mu_{f}^{2}$. From Eq. (33), we have

$$
\left(1 \mid \mathcal{Y}\left(\mu^{2} ; \boldsymbol{r}\right) \mathcal{O}_{J}(\boldsymbol{r})=\left(1 \mid \mathcal{O}_{J}(\boldsymbol{r}) \mathcal{X}_{1}\left(\mu^{2}\right) .\right.\right.
$$

Insert $1=\mathcal{X}_{1}\left(\mu_{f}^{2}\right) \mathcal{X}_{1}^{-1}\left(\mu_{f}^{2}\right)$ and use $\mathcal{X}_{1}^{-1}\left(\mu_{f}^{2}\right) \mathcal{X}_{1}\left(\mu^{2}\right)=$ $\mathcal{U}\left(\mu_{f}^{2}, \mu^{2}\right)$ from Eq. (27):

$$
\left(1 \mid \mathcal{Y}\left(\mu^{2} ; \boldsymbol{r}\right) \mathcal{O}_{J}(\boldsymbol{r})=\left(1 \mid \mathcal{O}_{J}(\boldsymbol{r}) \mathcal{X}_{1}\left(\mu_{f}^{2}\right) \mathcal{U}\left(\mu_{f}^{2}, \mu^{2}\right) .\right.\right.
$$

Since $\mu_{f}^{2}<Q_{J}^{2}(\boldsymbol{r})$, the operator $\mathcal{O}_{J}(\boldsymbol{r})$ does not resolve partons at the scale $\mu_{f}^{2}$. Thus $\mathcal{O}_{J}(\boldsymbol{r})$ commutes with $\mathcal{X}_{1}\left(\mu_{f}^{2}\right)$, giving us

$$
\left(1 \mid \mathcal{Y}\left(\mu^{2} ; \boldsymbol{r}\right) \mathcal{O}_{J}(\boldsymbol{r})=\left(1 \mid \mathcal{X}_{1}\left(\mu_{f}^{2}\right) \mathcal{O}_{J}(\boldsymbol{r}) \mathcal{U}\left(\mu_{f}^{2}, \mu^{2}\right) .\right.\right.
$$

Recall from Eq. (19) that $\left(1 \mid \mathcal{X}_{1}\left(\mu^{2}\right)=(1 \mid\right.$. This gives us

$$
\left(1 \mid \mathcal{Y}\left(\mu^{2} ; \boldsymbol{r}\right) \mathcal{O}_{J}(\boldsymbol{r})=\left(1 \mid \mathcal{O}_{J}(\boldsymbol{r}) \mathcal{U}\left(\mu_{f}^{2}, \mu^{2}\right) .\right.\right.
$$

That is, we compare two calculations. In the first calculation, we generate a parton shower down to a very small scale starting with any statistical state at a scale $\mu^{2}$. Then we measure $\mathcal{O}_{J}(\boldsymbol{r})$ inclusively using $\left(1 \mid \mathcal{O}_{J}(\boldsymbol{r})\right.$. In the second calculation, we first operate with $\mathcal{O}_{J}(\boldsymbol{r})$ on the state at scale $\mu^{2}$ then measure $\mathcal{Y}\left(\mu^{2} ; \boldsymbol{r}\right)$ inclusively using $\left(1 \mid \mathcal{Y}\left(\mu^{2} ; \boldsymbol{r}\right)\right.$. These two calculations give the same result.

\section{THE OPERATOR MAPPING $\mathbb{P}$}

In Sec. II D we defined an operator $\mathcal{V}\left(\mu^{2}\right)$ which is to obey Eq. (17), $\left(1 \mid \mathcal{V}\left(\mu^{2}\right)=\left(1 \mid \mathcal{F}_{0} \mathcal{D}\left(\mu^{2}\right) \mathcal{F}^{-1}\left(\mu^{2}\right)\right.\right.$. In Sec. III, we defined an operator $\mathcal{Y}\left(\mu^{2} ; \boldsymbol{r}\right)$ in the same way. In each case, we start with a singular operator $\mathcal{A}$ and we want to define a second, nonsingular, operator $\mathcal{B}$ with the property

$$
(1 \mid \mathcal{B}=(1 \mid \mathcal{A}
$$

When the operator $\mathcal{B}$ acts on an $m$-parton basis state $\left.\mid\left\{p, f, c, c^{\prime}, s, s^{\prime}\right\}_{m}\right)$, it is to leave the number of partons, their momenta, and their flavors unchanged. It may, however, act nontrivially on the colors and spins.

These requirements do not fully specify $\mathcal{B}$. We can be somewhat more definite by requiring that there be a linear mapping $\mathcal{A} \rightarrow \mathcal{B}$, which we write in the form

$$
\mathcal{B}=[\mathcal{A}]_{\mathbb{P}}
$$

This mapping must satisfy

$$
\left(1 \mid[\mathcal{A}]_{\mathbb{P}}=(1 \mid \mathcal{A}\right.
$$

and $[\mathcal{A}]_{\mathbb{P}}$ must leave $m$ and $\{p, f\}_{m}$ unchanged,

$$
\begin{aligned}
{\left.[\mathcal{A}]_{\mathbb{P}} \mid\left\{p, f, c, c^{\prime}, s, s^{\prime}\right\}_{m}\right)=} & \sum_{\left\{\hat{c}, \hat{c}^{\prime}, \hat{s}, \hat{s}^{\prime}\right\}_{m}} A\left(\{p, f\}_{m}\right)_{\left\{\hat{c}, \hat{c}^{\prime}, \hat{s}, \hat{s}^{\prime}\right\}_{m}}^{\left.\left.\{c,\}_{m}^{\prime},\right\}^{\prime}\right\}_{m}} \\
& \left.\times \mid\left\{p, f, \hat{c}, \hat{c}^{\prime}, \hat{s}, \hat{s}^{\prime}\right\}_{m}\right) .
\end{aligned}
$$

The requirement (43) is then a restriction on the spin and color matrix $A$, 


$$
\begin{aligned}
& \left(1|\mathcal{A}|\left\{p, f, c, c^{\prime}, s, s^{\prime}\right\}_{m}\right) \\
& =\sum_{\left\{\hat{c}, \hat{c}^{\prime}, \hat{s}, \hat{s}^{\prime}\right\}_{m}}\left\langle\left\{\hat{c}^{\prime}\right\}_{m} \mid\{\hat{c}\}_{m}\right\rangle\left\langle\left\{\hat{s}^{\prime}\right\}_{m} \mid\{\hat{s}\}_{m}\right\rangle \\
& \quad \times A\left(\{p, f\}_{m}\right)_{\left\{c \hat{c}, \hat{c}^{\prime}, \hat{s}, \hat{s}^{\prime}\right\}_{m}}^{\left\{c c^{\prime}, s,\right\}_{m}} .
\end{aligned}
$$

We can place another requirement on $[\cdots]_{\mathbb{P}}$ : if $\mathcal{A}$ has the property that it leaves $m$ and $\{p, f\}_{m}$ unchanged, then

$$
[\mathcal{A}]_{\mathbb{P}}=\mathcal{A} .
$$

One consequence of this is that $\left[[\mathcal{A}]_{\mathbb{P}}\right]_{\mathbb{P}}=[\mathcal{A}]_{\mathbb{P}}$.

These requirements do not fully specify the mapping $[\cdots]_{\mathbb{p}}$. For now we do not need to be more specific. However in Sec. XVIC we provide an example (without spin) that is useful for the analysis of a first order shower.

We will find that the combination $\mathcal{A}-[\mathcal{A}]_{\mathbb{P}}$ appears frequently in formulas. It useful to define an operation $[\cdots]_{1-\mathbb{P}}$ by

$$
[\mathcal{A}]_{1-\mathbb{P}}=\mathcal{A}-[\mathcal{A}]_{\mathbb{P}} .
$$

\section{GENERATOR OF SHOWER}

We now turn to a more detailed study of the operator $\mathcal{U}\left(\mu_{f}^{2}, \mu_{\mathrm{H}}^{2}\right)$ that creates a parton shower between a hard scale $\mu_{\mathrm{H}}^{2}$ and a small, cutoff scale $\mu_{f}^{2}$. The generator of this shower evolution is the operator

$$
\frac{1}{\mu^{2}} \mathcal{S}\left(\mu^{2}\right)=\left[\mathcal{X}_{1}^{-1}\left(\mu_{\mathrm{R}}^{2}, \mu^{2}\right) \frac{\partial}{\partial \mu^{2}} \mathcal{X}_{1}\left(\mu_{\mathrm{R}}^{2}, \mu^{2}\right)\right]_{\mu_{\mathrm{R}}^{2}=\kappa_{\mathrm{R}} \mu^{2}} .
$$

Here, we differentiate with respect to the shower scale. Because of Eq. (10) [with the use of Eqs. (17) and (18)], this is the same as

$$
\frac{1}{\mu^{2}} \mathcal{S}\left(\mu^{2}\right)=\mathcal{X}_{1}^{-1}\left(\mu^{2}\right) \frac{\partial}{\partial \mu^{2}} \mathcal{X}_{1}\left(\mu^{2}\right)
$$

Because of Eq. (19),

$$
\left(1 \mid \mathcal{S}\left(\mu^{2}\right)=0 .\right.
$$

Equation (51) gives us a differential equation for $\mathcal{U}$

$$
\mu^{2} \frac{\partial}{\partial \mu^{2}} \mathcal{U}\left(\mu_{2}^{2}, \mu^{2}\right)=\mathcal{U}\left(\mu_{2}^{2}, \mu^{2}\right) \mathcal{S}\left(\mu^{2}\right)
$$

We use the notation

$$
\mathcal{U}\left(\mu_{2}^{2}, \mu_{1}^{2}\right)=\mathbb{T} \exp \left(\int_{\mu_{2}^{2}}^{\mu_{1}^{2}} \frac{d \mu^{2}}{\mu^{2}} \mathcal{S}\left(\mu^{2}\right)\right)
$$

to represent the solution of this equation. Here $\mathbb{T}$ indicates the instruction to order the operators $\mathcal{S}\left(\mu^{2}\right)$ with the smallest $\mu^{2}$ to the left.

\section{THE THRESHOLD FACTOR}

In Sec. II D we have defined an operator $\mathcal{V}\left(\mu_{\mathrm{R}}^{2}, \mu_{\mathrm{S}}^{2}\right)$. With our notation in Eq. (21) for the scale dependence of $\mathcal{V}$, the crucial property given in Eq. (17) can be written

$$
\left(1 \mid \mathcal{V}(\mu)=\left(1 \mid \mathcal{F}_{0} \mathcal{D}\left(\mu^{2}\right) \mathcal{F}^{-1}(\mu) .\right.\right.
$$

In Eq. (29) or Eq. (38), the perturbative expansion of $\mathcal{V}\left(\mu_{\mathrm{H}}^{2}\right)$ contains large logarithms $[18,25,26]$. These are the much studied threshold logarithms [29]. We sum the threshold logarithms by writing $\mathcal{V}\left(\mu_{\mathrm{H}}^{2}\right)$ as an exponential. Define

$$
\mathcal{U}_{\mathcal{V}}\left(\mu_{2}^{2}, \mu_{1}^{2}\right)=\mathcal{V}^{-1}\left(\mu_{2}^{2}\right) \mathcal{V}\left(\mu_{1}^{2}\right)
$$

Then $\mathcal{V}\left(\mu_{\mathrm{H}}^{2}\right)$ can be written as

$$
\mathcal{V}\left(\mu_{\mathrm{H}}^{2}\right)=\mathcal{V}\left(\mu_{f}^{2}\right) \mathcal{U}_{\mathcal{V}}\left(\mu_{f}^{2}, \mu_{\mathrm{H}}^{2}\right) .
$$

Define a generator operator $\mathcal{S}_{\mathcal{V}}\left(\mu^{2}\right)$ by

$$
\frac{1}{\mu^{2}} \mathcal{S}_{\mathcal{V}}\left(\mu^{2}\right)=\mathcal{V}^{-1}\left(\mu^{2}\right) \frac{d \mathcal{V}\left(\mu^{2}\right)}{d \mu^{2}}
$$

Then $\mathcal{U}_{\mathcal{V}}\left(\mu_{2}^{2}, \mu_{1}^{2}\right)$ is the solution of the differential equation

$$
\mu^{2} \frac{\partial}{\partial \mu^{2}} \mathcal{U}_{\mathcal{V}}\left(\mu_{2}^{2}, \mu^{2}\right)=\mathcal{U}_{\mathcal{V}}\left(\mu_{2}^{2}, \mu^{2}\right) \mathcal{S}_{\mathcal{V}}\left(\mu^{2}\right)
$$

We write the solution of this equation as

$$
\mathcal{U}_{\mathcal{V}}\left(\mu_{f}^{2}, \mu_{\mathrm{H}}^{2}\right)=\mathbb{T} \exp \left(\int_{\mu_{f}^{2}}^{\mu_{\mathrm{H}}^{2}} \frac{d \mu^{2}}{\mu^{2}} \mathcal{S}_{\mathcal{V}}\left(\mu^{2}\right)\right) .
$$

As long as we expand the running coupling $\alpha_{s}$ in Eq. (60) to some finite order in $\alpha_{s}\left(\mu_{\mathrm{H}}^{2}\right)$, the integral in Eq. (60) is convergent in the limit $\mu_{f}^{2} \rightarrow 0$ [18]. Thus $\mathcal{V}\left(\mu^{2}\right)$ at small scales is almost the unit operator,

$$
\mathcal{V}\left(\mu_{f}^{2}\right) \approx 1
$$

That is

$$
\mathcal{V}\left(\mu_{\mathrm{H}}^{2}\right) \approx \mathcal{U}_{\mathcal{V}}\left(\mu_{f}^{2}, \mu_{\mathrm{H}}^{2}\right) .
$$

\section{PERTURBATIVE EXPANSIONS}

The operator $\mathcal{S}\left(\mu^{2}\right)$ can be expanded in powers of $\alpha_{s}\left(\mu_{\mathrm{R}}^{2}\right)=\alpha_{s}\left(\kappa_{\mathrm{R}} \mu^{2}\right)$ : 


$$
\mathcal{S}\left(\mu^{2}\right)=\sum_{n=1}^{\infty}\left[\frac{\alpha_{s}\left(\kappa_{\mathrm{R}} \mu^{2}\right)}{2 \pi}\right]^{n} \mathcal{S}^{(n)}\left(\mu^{2}\right)
$$

In the general theory from Ref. [18], $\mathcal{S}\left(\mu^{2}\right)$ is constructed from the singular operator $\mathcal{D}\left(\mu_{\mathrm{R}}^{2}, \mu_{\mathrm{S}}^{2}\right)$. If we use only the first order part $\mathcal{D}^{(1)}\left(\mu_{\mathrm{R}}^{2}, \mu_{\mathrm{S}}^{2}\right)$ of $\mathcal{D}$ because that is all we know, then all we get is $\mathcal{S}^{(1)}\left(\mu^{2}\right)$. However, in a practical parton shower program (such as the $\Lambda$-ordered DEDUCTOR), one often takes a guess at approximate higher order contributions $\mathcal{S}^{(n)}$. The approximate form is obtained by changing the argument of $\alpha_{s}$ in the splitting functions to $\kappa_{\mathrm{R}} k_{\mathrm{T}}^{2}$ and, additionally, making a special choice for $\kappa_{\mathrm{R}}$. Expanding $\alpha_{s}\left(\kappa_{\mathrm{R}} k_{\mathrm{T}}^{2}\right)$ in powers of $\alpha_{s}\left(\kappa_{\mathrm{R}} \mu^{2}\right)$ then produces contributions $\mathcal{S}^{(n)}\left(\mu^{2}\right)$ for $n>1$.

In DEDUCTOR, the first order contribution has three parts $[18,25]$ :

$$
\mathcal{S}^{(1)}\left(\mu^{2}\right)=\mathcal{S}^{(1,0)}\left(\mu^{2}\right)-\left[\mathcal{S}^{(1,0)}\left(\mu^{2}\right)\right]_{\mathbb{P}}+\mathrm{i} \pi \mathcal{S}_{\mathrm{i} \pi}^{(0,1)}\left(\mu^{2}\right) .
$$

The operator $\mathcal{S}^{(1,0)}\left(\mu^{2}\right)$ describes parton splitting, changing an $m$ parton state to an $m+1$ parton state. The operator $\left[\mathcal{S}^{(1,0)}\left(\mu^{2}\right)\right]_{\mathbb{P}}$ leaves $m$ and $\{p, f\}_{m}$ in an $m$ parton state unchanged, although it can modify the color state. ${ }^{2}$ In a leading color parton shower, the color is unchanged and the eigenvalue of this operator then gives the order $\alpha_{\mathrm{s}}$ contribution to the integrand in the exponent of the Sudakov factor that represents the probability not to split between two scales. The final operator, $\mathcal{S}_{\mathrm{i} \pi}^{(0,1)}\left(\mu^{2}\right)$, leaves $m$ and $\{p, f\}_{m}$ unchanged. It gives the imaginary part of virtual graphs [18,25] and obeys $\left(1 \mid \mathcal{S}_{\mathrm{i} \pi}^{(0,1)}\left(\mu^{2}\right)=0\right.$.

The operator $\mathcal{S}_{\mathcal{V}}\left(\mu^{2}\right)$ has a perturbative expansion

$$
\mathcal{S}_{\mathcal{V}}\left(\mu^{2}\right)=\sum_{n=1}^{\infty}\left[\frac{\alpha_{\mathrm{S}}\left(\kappa_{\mathrm{R}} \mu^{2}\right)}{2 \pi}\right]^{n} \mathcal{S}_{\mathcal{V}}^{(n)}\left(\mu^{2}\right)
$$

The first order operator $\mathcal{S}_{\mathcal{V}}^{(1)}\left(\mu^{2}\right)$ has the form [18,25,26]

$$
\begin{aligned}
\mathcal{S}_{\mathcal{V}}^{(1)}\left(\mu^{2}\right)= & {\left[\mathcal{S}^{(1,0)}\left(\mu^{2}\right)\right]_{\mathbb{P}}+\operatorname{Re} \mathcal{S}_{\text {pert }}^{(0,1)}\left(\mu^{2}\right) } \\
& -\left[\mathcal{F}\left(\mu^{2}\right) \circ \mathcal{P}^{(1)}\right] \mathcal{F}^{-1}\left(\mu^{2}\right) .
\end{aligned}
$$

Here $\left[\mathcal{S}^{(1,0)}\left(\mu^{2}\right)\right]_{\mathbb{P}}$ is proportional to the integral of the first order splitting function over the splitting variables and appears also in Eq. (64). In the third term, $\left[\mathcal{F}\left(\mu^{2}\right) \circ \mathcal{P}^{(1)}\right]$ denotes the convolution of $\mathcal{F}\left(\mu^{2}\right)$ with the first order PDF evolution kernel $\mathcal{P}^{(1)}$. In the second term,

$$
\frac{1}{\mu^{2}} \mathcal{S}_{\text {pert }}^{(0,1)}\left(\mu^{2}\right)=\left[\frac{\partial}{\partial \mu^{2}} \mathcal{D}^{(0,1)}\left(\mu_{\mathrm{R}}^{2}, \mu^{2}\right)\right]_{\mu_{\mathrm{R}}^{2}=\kappa_{\mathrm{R}} \mu^{2}}
$$

${ }^{2}\left[\mathcal{S}^{(1,0)}\left(\mu^{2}\right)\right]_{\mathbb{P}}$ was denoted by $\left[\mathcal{F}\left(\mu^{2}\right) \circ \overline{\mathcal{S}}^{(1,0)}\left(\mu^{2}\right)\right] \mathcal{F}^{-1}\left(\mu^{2}\right)$ in Ref. [18]. is the derivative with respect to the shower scale of the singular operator for a one loop virtual graph. It is sometimes assumed that the effect of virtual graphs and PDF evolution cancels the integral over the splitting variables of parton splitting [30]. However, this cancellation is not complete, so that the effect of $\mathcal{S}_{\mathcal{V}}^{(1)}\left(\mu^{2}\right)$ is quite important [26,30].

\section{GENERATOR OF $\mathcal{Y}$}

We now turn to a more detailed study of the operator $\mathcal{Y}\left(\mu^{2} ; \boldsymbol{r}\right)$. This operator sums logarithms, so we want to write it as an exponential. Define

$$
\frac{1}{\mu^{2}} \mathcal{S}_{\mathcal{Y}}\left(\mu^{2} ; \boldsymbol{r}\right)=\mathcal{Y}^{-1}\left(\mu^{2} ; \boldsymbol{r}\right) \frac{d}{d \mu^{2}} \mathcal{Y}\left(\mu^{2} ; \boldsymbol{r}\right)
$$

This gives us a differential equation for $\mathcal{Y}\left(\mu^{2} ; \boldsymbol{r}\right)$

$$
\mu^{2} \frac{d}{d \mu^{2}} \mathcal{Y}\left(\mu^{2} ; \boldsymbol{r}\right)=\mathcal{Y}\left(\mu^{2} ; \boldsymbol{r}\right) \mathcal{S}_{\mathcal{Y}}\left(\mu^{2} ; \boldsymbol{r}\right) \text {. }
$$

We solve this equation with a boundary condition at the shower cutoff scale $\mu_{\mathrm{f}}^{2}$ :

$\mathcal{Y}\left(\mu^{2} ; \boldsymbol{r}\right)=\mathcal{Y}\left(\mu_{f}^{2} ; \boldsymbol{r}\right)+\int_{\mu_{f}^{2}}^{\mu^{2}} \frac{d \bar{\mu}^{2}}{\bar{\mu}^{2}} \mathcal{Y}\left(\mu^{2} ; \boldsymbol{r}\right) \mathcal{S}_{\mathcal{Y}}\left(\mu^{2} ; \boldsymbol{r}\right)$.

Recall the defining condition Eq. (33) for $\mathcal{Y}\left(\mu^{2} ; \boldsymbol{r}\right)$. At $\mu^{2}=\mu_{f}^{2}$ this condition is

$$
\left(1 \mid \mathcal{Y}\left(\mu_{f}^{2} ; \boldsymbol{r}\right)=\left(1 \mid \mathcal{O}_{J}(\boldsymbol{r}) \mathcal{X}_{1}\left(\mu_{f}^{2}\right) \mathcal{O}_{J}^{-1}(\boldsymbol{r})\right.\right.
$$

The measurement operator $\mathcal{O}_{J}(\boldsymbol{r})$ is an infrared safe operator that is not sensitive to parton scales below a scale $Q_{J}^{2}(\boldsymbol{r})$. We suppose that $\mu_{f}^{2}<Q_{J}^{2}(\boldsymbol{r})$. Then $\mathcal{O}_{J}(\boldsymbol{r})$ commutes with $\mathcal{X}_{1}\left(\mu_{f}^{2}\right)$ and we can use Eq. (19), which gives us $\left(1 \mid \mathcal{Y}\left(\mu_{f}^{2} ; \boldsymbol{r}\right)=(1 \mid\right.$. Thus we can define

$$
\mathcal{Y}\left(\mu_{f}^{2} ; \boldsymbol{r}\right)=1 .
$$

This allows us to write the solution of Eq. (69) as

$$
\mathcal{Y}\left(\mu^{2} ; \boldsymbol{r}\right)-1=\int_{\mu_{f}^{2}}^{\mu^{2}} \frac{d \bar{\mu}^{2}}{\bar{\mu}^{2}} \mathcal{Y}\left(\bar{\mu}^{2} ; \boldsymbol{r}\right) \mathcal{S}_{\mathcal{Y}}\left(\bar{\mu}^{2} ; \boldsymbol{r}\right) .
$$

We can also write $\mathcal{Y}\left(\mu_{\mathrm{H}}^{2} ; \boldsymbol{r}\right)$ as a hardness-ordered exponential,

$$
\mathcal{Y}\left(\mu_{\mathrm{H}}^{2} ; \boldsymbol{r}\right)=\mathbb{T} \exp \left(\int_{\mu_{f}^{2}}^{\mu_{\mathrm{H}}^{2}} \frac{d \mu^{2}}{\mu^{2}} \mathcal{S}_{\mathcal{Y}}\left(\mu^{2} ; \boldsymbol{r}\right)\right) .
$$

To find the generator $\mathcal{S}_{\mathcal{Y}}\left(\mu^{2} ; \boldsymbol{r}\right)$ we start with Eq. (42), which we write as 


$$
\left(1 \mid \mathcal{Y}\left(\mu^{2} ; \boldsymbol{r}\right)=\left(1 \mid \mathcal{O}_{J}(\boldsymbol{r}) \mathcal{U}\left(\mu_{f}^{2}, \mu^{2}\right) \mathcal{O}_{J}^{-1}(\boldsymbol{r})\right.\right.
$$

This applies for $\mathcal{U}\left(\mu_{f}^{2}, \mu^{2}\right)$ and $\mathcal{Y}\left(\mu^{2} ; \boldsymbol{r}\right)$ evaluated at any order $K$ of perturbation theory, with corrections of order $\alpha_{s}^{K+1}$. We can also use Eq. (75) if $\mathcal{U}\left(\mu_{f}^{2}, \mu^{2}\right)$ is an approximate shower evolution operator as defined in a particular parton shower algorithm. In this case, the shower splitting operator $\mathcal{S}\left(\mu^{2}\right)$ may be based on lowest order perturbation theory. If $\mathcal{U}\left(\mu_{f}^{2}, \mu^{2}\right)$ is approximate, then Eq. (75) defines the corresponding approximate operator $\mathcal{Y}\left(\mu^{2} ; \boldsymbol{r}\right)$ and Eq. (68) defines the corresponding approximate generator $\mathcal{S}_{\mathcal{Y}}\left(\mu^{2} ; \boldsymbol{r}\right)$.

We can differentiate Eq. (75) with respect to $\mu^{2}$ and use Eq. (69) for the derivative of $\mathcal{Y}$ and Eq. (53) for the derivative of $\mathcal{U}\left(\mu_{f}^{2}, \mu^{2}\right)$,

$$
\left(1 \mid \mathcal{Y}\left(\mu^{2} ; \boldsymbol{r}\right) \mathcal{S}_{\mathcal{Y}}\left(\mu^{2} ; \boldsymbol{r}\right)=\left(1 \mid \mathcal{O}_{J}(\boldsymbol{r}) \mathcal{U}\left(\mu_{f}^{2}, \mu^{2}\right) \mathcal{S}\left(\mu^{2}\right) \mathcal{O}_{J}^{-1}(\boldsymbol{r})\right.\right.
$$

We insert $1=\mathcal{O}_{J}^{-1}(\boldsymbol{r}) \mathcal{O}_{J}(\boldsymbol{r})$ to give

$$
\begin{aligned}
\left(1 \mid \mathcal{Y}\left(\mu^{2} ; \boldsymbol{r}\right) \mathcal{S}_{\mathcal{Y}}\left(\mu^{2} ; \boldsymbol{r}\right)=\right. & \left(1 \mid \mathcal{O}_{J}(\boldsymbol{r}) \mathcal{U}\left(\mu_{f}^{2}, \mu^{2}\right) \mathcal{O}_{J}^{-1}(\boldsymbol{r})\right. \\
& \times \mathcal{O}_{J}(\boldsymbol{r}) \mathcal{S}\left(\mu^{2}\right) \mathcal{O}_{J}^{-1}(\boldsymbol{r}) .
\end{aligned}
$$

Using Eq. (75) then gives us

$$
\left(1 \mid \mathcal{Y}\left(\mu^{2} ; \boldsymbol{r}\right) \mathcal{S}_{\mathcal{Y}}\left(\mu^{2} ; \boldsymbol{r}\right)=\left(1 \mid \mathcal{Y}\left(\mu^{2} ; \boldsymbol{r}\right) \mathcal{O}_{J}(\boldsymbol{r}) \mathcal{S}\left(\mu^{2}\right) \mathcal{O}_{J}^{-1}(\boldsymbol{r})\right.\right.
$$

The operators $\mathcal{Y}$ and $\mathcal{S}_{\mathcal{Y}}$ are nonsingular operators that leave the number of partons and their momenta and flavors unchanged. Thus we can use the mapping $[\cdots]_{\mathbb{P}}$ defined in Sec. IV to write this as

$$
\mathcal{Y}\left(\mu^{2} ; \boldsymbol{r}\right) \mathcal{S}_{\mathcal{Y}}\left(\mu^{2} ; \boldsymbol{r}\right)=\left[\mathcal{Y}\left(\mu^{2} ; \boldsymbol{r}\right) \mathcal{O}_{J}(\boldsymbol{r}) \mathcal{S}\left(\mu^{2}\right) \mathcal{O}_{J}^{-1}(\boldsymbol{r})\right]_{\mathbb{P}}
$$

The expansion of $\mathcal{Y}$ in powers of $\alpha_{\mathrm{s}}$ starts at $\mathcal{Y}=1+\cdots$, so a useful way to write this is

$$
\begin{aligned}
\mathcal{S}_{\mathcal{Y}}\left(\mu^{2} ; \boldsymbol{r}\right)= & {\left[\mathcal{Y}\left(\mu^{2} ; \boldsymbol{r}\right) \mathcal{O}_{J}(\boldsymbol{r}) \mathcal{S}\left(\mu^{2}\right) \mathcal{O}_{J}^{-1}(\boldsymbol{r})\right]_{\mathbb{P}} } \\
& -\left\{\mathcal{Y}\left(\mu^{2} ; \boldsymbol{r}\right)-1\right\} \mathcal{S}_{\mathcal{Y}}\left(\mu^{2} ; \boldsymbol{r}\right) .
\end{aligned}
$$

Now we can use Eqs. (80) and (73) recursively to generate $\mathcal{S}_{\mathcal{Y}}$ and $\mathcal{Y}$ in powers of $\alpha_{s}$. We write

$$
\begin{aligned}
\mathcal{S}_{\mathcal{Y}}\left(\mu^{2} ; \boldsymbol{r}\right) & =\sum_{n=1}^{\infty}\left[\frac{\alpha_{\mathrm{s}}\left(\kappa_{\mathrm{R}} \mu^{2}\right)}{2 \pi}\right]^{n} \mathcal{S}_{\mathcal{Y}}^{(n)}\left(\mu^{2} ; \boldsymbol{r}\right), \\
\mathcal{Y}\left(\mu^{2} ; \boldsymbol{r}\right) & =\sum_{n=0}^{\infty}\left[\frac{\alpha_{\mathrm{S}}\left(\kappa_{\mathrm{R}} \mu^{2}\right)}{2 \pi}\right]^{n} \mathcal{Y}^{(n)}\left(\mu^{2} ; \boldsymbol{r}\right),
\end{aligned}
$$

with

$$
\mathcal{Y}^{(0)}\left(\mu^{2} ; \boldsymbol{r}\right)=1 .
$$

For $\mathcal{S}_{\mathcal{Y}}$, Eq. (80) gives

$$
\begin{aligned}
\mathcal{S}_{\mathcal{Y}}^{(n)}\left(\mu^{2} ; \boldsymbol{r}\right)= & {\left[\mathcal{O}_{J}(\boldsymbol{r}) \mathcal{S}^{(n)}\left(\mu^{2}\right) \mathcal{O}_{J}^{-1}(\boldsymbol{r})\right]_{\mathbb{P}} } \\
& +\sum_{j=1}^{n-1}\left[\mathcal{Y}^{(n-j)}\left(\mu^{2} ; \boldsymbol{r}\right) \mathcal{O}_{J}(\boldsymbol{r}) \mathcal{S}^{(j)}\left(\mu^{2}\right) \mathcal{O}_{J}^{-1}(\boldsymbol{r})\right]_{\mathbb{P}} \\
& -\sum_{j=1}^{n-1} \mathcal{Y}^{(n-j)}\left(\mu^{2} ; \boldsymbol{r}\right) \mathcal{S}_{\mathcal{Y}}^{(j)}\left(\mu^{2} ; \boldsymbol{r}\right)
\end{aligned}
$$

This gives us $\mathcal{S}_{\mathcal{Y}}^{(n)}$ if we know $\mathcal{S}_{\mathcal{Y}}^{(j)}$ for $j<n$ and $\mathcal{Y}^{(k)}$ for $k<n$.

For $\mathcal{Y}$ we use Eq. (73), in which an integration over an intermediate scale $\bar{\mu}^{2}$ appears. We can expand $\alpha_{s}\left(\kappa_{\mathrm{R}} \bar{\mu}^{2}\right)$ in powers of $\alpha_{s}\left(\kappa_{\mathrm{R}} \mu^{2}\right)$ in the form

$$
\left[\frac{\alpha_{s}\left(\kappa_{\mathrm{R}} \bar{\mu}^{2}\right)}{2 \pi}\right]^{k}=\sum_{n=k}^{\infty} \gamma\left(k, n ; \bar{\mu}^{2} / \mu^{2}\right)\left[\frac{\alpha_{s}\left(\kappa_{\mathrm{R}} \mu^{2}\right)}{2 \pi}\right]^{n},
$$

with coefficients $\gamma$ derived from the QCD $\beta$-function. Using this expansion in Eq. (73), we obtain

$$
\begin{aligned}
\mathcal{Y}^{(n)}\left(\mu^{2} ; \boldsymbol{r}\right)= & \int_{\mu_{f}^{2}}^{\mu^{2}} \frac{d \bar{\mu}^{2}}{\bar{\mu}^{2}} \sum_{j=1}^{n} \sum_{k=0}^{n-j} \gamma\left(k+j, n ; \bar{\mu}^{2} / \mu^{2}\right) \\
& \times \mathcal{Y}^{(k)}\left(\bar{\mu}^{2} ; \boldsymbol{r}\right) \mathcal{S}_{\mathcal{Y}}^{(j)}\left(\bar{\mu}^{2} ; \boldsymbol{r}\right) .
\end{aligned}
$$

This gives us $\mathcal{Y}^{(n)}$ if we know $\mathcal{Y}^{(k)}$ for $k<n$ and $\mathcal{S}_{\mathcal{Y}}^{(j)}$ for $j \leq n$.

These recursion relations successively generate $\mathcal{S}_{\mathcal{Y}}^{(1)}$, $\mathcal{Y}^{(1)}, \mathcal{S}_{\mathcal{Y}}^{(2)}, \mathcal{Y}^{(2)}, \ldots$. The first order terms are

$$
\mathcal{S}_{\mathcal{Y}}^{(1)}\left(\mu^{2} ; \boldsymbol{r}\right)=\left[\mathcal{O}_{J}(\boldsymbol{r}) \mathcal{S}^{(1)}\left(\mu^{2}\right) \mathcal{O}_{J}^{-1}(\boldsymbol{r})\right]_{\mathbb{P}}
$$

and

$$
\mathcal{Y}^{(1)}\left(\mu^{2} ; \boldsymbol{r}\right)=\int_{\mu_{f}^{2}}^{\mu^{2}} \frac{d \bar{\mu}^{2}}{\bar{\mu}^{2}}\left[\mathcal{O}_{J}(\boldsymbol{r}) \mathcal{S}^{(1)}\left(\bar{\mu}^{2}\right) \mathcal{O}_{J}^{-1}(\boldsymbol{r})\right]_{\mathbb{P}} .
$$

\section{USING $\mathcal{Y}$}

We now outline how the operator $\mathcal{Y}\left(\mu^{2} ; \boldsymbol{r}\right)$ can be used. This operator is the key to calculating an observable cross section $\sigma_{J}(\boldsymbol{r})$ according to a parton shower algorithm. The operator $\mathcal{O}_{J}(\boldsymbol{r})$ that defines this cross section must be infrared safe. That is, there is a scale $Q_{J}^{2}(\boldsymbol{r})$ such that $\sigma_{J}(\boldsymbol{r})$ does not resolve parton splittings at scales $\mu^{2}$ smaller than $Q_{J}^{2}(\boldsymbol{r})$. In order to define $\mathcal{Y}\left(\mu^{2} ; \boldsymbol{r}\right)$, the inverse operator $\mathcal{O}_{J}^{-1}(\boldsymbol{r})$ must exist. The anticipated use case is that there is a 
distribution of direct interest that involves large logarithms and the logarithms can be summed analytically by taking an integral transform of the distribution that depends on parameters $\boldsymbol{r}$. Then $\sigma_{J}(\boldsymbol{r})$ represents the value of this integral transform. Starting in Sec. XI, we examine an important example, the thrust distribution in electronpositron annihilation. Then one uses the Laplace transform of the thrust distribution and $\boldsymbol{r}$ is the Laplace parameter $\nu$.

In the applications that we have in mind, the perturbative expansion of $\sigma_{J}(\boldsymbol{r})$ contains powers of a large logarithm $L(\boldsymbol{r})$ when the parameter or parameters $\boldsymbol{r}$ approach some limit. Typically, we have

$$
\sigma_{J}(\boldsymbol{r})=c_{0}\left\{1+\sum_{n=1}^{\infty} \sum_{j=0}^{2 n} c(n, j) \alpha_{s}^{n}\left(\mu_{\mathrm{H}}^{2}\right) L^{j}(\boldsymbol{r})\right\} .
$$

In favorable cases, there is an analytical formula that sums these logarithms in the form

$$
\sigma_{J}(\boldsymbol{r})=c_{0} \exp \left(\sum_{n=1}^{\infty} \sum_{j=0}^{n+1} d(n, j) \alpha_{s}^{n}\left(\mu_{\mathrm{H}}^{2}\right) L^{j}(\boldsymbol{r})\right) .
$$

It is crucial here that the maximum power of $L$ at order $\alpha_{s}^{n}$ is $j=n+1$, not $2 n$. We can say that a $\sigma_{J}(\boldsymbol{r})$ with this property exponentiates. One never knows all of the coefficients $d(n, j)$, but when the coefficients for $j=n+1$ are known, we can say that the formula sums the logarithms at the leading-log (LL) level. When the coefficients for $j=n$ are also known, we can say that the formula sums the logarithms at the next-to-leading-log (NLL) level.

In some important cases, the color space for the partons involved in the hard scattering process is trivial. For instance, for shape observables in electron-positron annihilation, there is only one color basis vector for the $q \bar{q}$ state in $e^{+} e^{-} \rightarrow q \bar{q}$. Then the coefficients $d(n, j)$ are numbers. The initial partonic state in hadron-hadron scattering has a nontrivial color structure. Then the coefficients $d(n, j)$ may be integrals of matrices in the parton color space, with some specification for the ordering of noncommuting matrices in the exponent.

What does a parton shower algorithm say about $\sigma_{J}(\boldsymbol{r})$ ? Different parton showers can give different answers, so we should have a particular parton shower algorithm in mind.

We have seen that there are two ways to express $\sigma_{J}(\boldsymbol{r})$ as given by a parton shower. First, we can use Eq. (29),

$$
\sigma_{J}(\boldsymbol{r})=\left(1\left|\mathcal{O}_{J}(\boldsymbol{r}) \mathcal{U}\left(\mu_{f}^{2}, \mu_{\mathrm{H}}^{2}\right) \mathcal{V}\left(\mu_{\mathrm{H}}^{2}\right) \mathcal{F}\left(\mu_{\mathrm{H}}^{2}\right)\right| \rho_{\mathrm{H}}\left(\mu_{\mathrm{H}}^{2}\right)\right) .
$$

Typically the splitting operator $\mathcal{S}$ in $\mathcal{U}\left(\mu_{f}^{2}, \mu_{\mathrm{H}}^{2}\right)$ is based on lowest order perturbation theory, as discussed at the beginning of Sec. VII. Additionally, $\mathcal{V}\left(\mu_{\mathrm{H}}^{2}\right)$ is present in DEDUCTOR, but for many parton shower algorithms $\mathcal{V}=1$. Equation (90) says to run the parton shower to its cutoff scale and then measure the observable by applying $\left(1 \mid \mathcal{O}_{J}(\boldsymbol{r})\right.$. The perturbative expansion of this result has the form (88), but not directly the form (89). One can run the corresponding parton shower event generator to obtain a numerical result with statistical errors and other numerical errors. Even with errors, it is possible $[2,16]$ to use numerical results from Eq. (90) to check these results against a known QCD analytic result, as we will see later in this paper.

The second way to express $\sigma_{J}(\boldsymbol{r})$ as given by a parton shower is contained in Eq. (38),

$$
\sigma_{J}(\boldsymbol{r})=\left(1\left|\mathcal{Y}\left(\mu_{\mathrm{H}}^{2} ; \boldsymbol{r}\right) \mathcal{V}\left(\mu_{\mathrm{H}}^{2}\right) \mathcal{F}\left(\mu_{\mathrm{H}}^{2}\right) \mathcal{O}_{J}(\boldsymbol{r})\right| \rho_{\mathrm{H}}\left(\mu_{\mathrm{H}}^{2}\right)\right),
$$

with $\mathcal{Y}\left(\mu_{\mathrm{H}}^{2} ; \boldsymbol{r}\right)$ given by Eq. (74) as an exponential of a generator $\mathcal{S}_{\mathcal{Y}}$

$$
\mathcal{Y}\left(\mu_{\mathrm{H}}^{2} ; \boldsymbol{r}\right)=\mathbb{T} \exp \left(\int_{\mu_{f}^{2}}^{\mu_{\mathrm{H}}^{2}} \frac{d \mu^{2}}{\mu^{2}} \mathcal{S}_{\mathcal{Y}}\left(\mu^{2} ; \boldsymbol{r}\right)\right) .
$$

The operator $\mathcal{S}_{\mathcal{Y}}$ is obtained from the shower generator $\mathcal{S}$ using Eqs. (83) and (85). This second expression for $\sigma_{J}(\boldsymbol{r})$ gives exactly the same $\sigma_{J}(\boldsymbol{r})$ as given by Eq. (90). However, now the logarithms $L$ appear in the exponent in $\mathcal{S}_{\mathcal{Y}}$. Thus we have a representation that is very close to the representation in Eq. (89).

The exponent in $\mathcal{Y}$ is $^{3}$

$$
\mathcal{I}(\boldsymbol{r})=\int_{\mu_{f}^{2}}^{\mu_{\mathrm{H}}^{2}} \frac{d \mu^{2}}{\mu^{2}} \mathcal{S}_{\mathcal{Y}}\left(\mu^{2} ; \boldsymbol{r}\right) .
$$

If we use the perturbative expansion of $\mathcal{S}_{\mathcal{Y}}$, this is

$$
\mathcal{I}(\boldsymbol{r})=\sum_{n=1}^{\infty} \int_{\mu_{f}^{2}}^{\mu_{\mathrm{H}}^{2}} \frac{d \mu^{2}}{\mu^{2}}\left[\frac{\alpha_{\mathrm{s}}\left(\kappa_{\mathrm{R}} \mu^{2}\right)}{2 \pi}\right]^{n} \mathcal{S}_{\mathcal{Y}}^{(n)}\left(\mu^{2} ; \boldsymbol{r}\right) .
$$

For $\mathcal{S}_{\mathcal{Y}}^{(n)}$ we have

$\mathcal{S}_{\mathcal{Y}}^{(n)}\left(\mu^{2} ; \boldsymbol{r}\right)=\left[\mathcal{O}_{J}(\boldsymbol{r}) \mathcal{S}^{(n)}\left(\mu^{2}\right) \mathcal{O}_{J}^{-1}(\boldsymbol{r})\right]_{\mathbb{P}}+\Delta \mathcal{S}_{\mathcal{Y}}^{(n)}\left(\mu^{2} ; \boldsymbol{r}\right)$.

Here $\left[\mathcal{O}_{J}(\boldsymbol{r}) \mathcal{S}^{(n)}\left(\mu^{2}\right) \mathcal{O}_{J}^{-1}(\boldsymbol{r})\right]_{\mathbb{P}}$ is the first term in Eq. (83) and is the only term for $n=1$. For $n>1, \Delta \mathcal{S}_{\mathcal{Y}}^{(n)}\left(\mu^{2} ; \boldsymbol{r}\right)$ is everything else in Eq. (83).

We can now expand $\mathcal{I}(\boldsymbol{r})$ in powers of $\alpha_{s}\left(\mu_{\mathrm{H}}^{2}\right)$. The perturbative coefficients will contain powers of the large logarithm $L(\boldsymbol{r})$. Let us divide $\mathcal{I}(\boldsymbol{r})$ into two pieces

$$
\mathcal{I}(\boldsymbol{r})=\mathcal{I}_{0}(\boldsymbol{r})+\Delta \mathcal{I}(\boldsymbol{r}),
$$

where

\footnotetext{
${ }^{3}$ This includes, possibly, ordering of operators or matrices in the exponential. For simplicity, we ignore questions of ordering here.
} 


$$
\begin{aligned}
\mathcal{I}_{0}(\boldsymbol{r})= & \sum_{n=1}^{\infty} \int_{\mu_{f}^{2}}^{\mu_{\mathrm{H}}^{2}} \frac{d \mu^{2}}{\mu^{2}}\left[\frac{\alpha_{s}\left(\kappa_{\mathrm{R}} \mu^{2}\right)}{2 \pi}\right]^{n} \\
& \times\left[\mathcal{O}_{J}(\boldsymbol{r}) \mathcal{S}^{(n)}\left(\mu^{2}\right) \mathcal{O}_{J}^{-1}(\boldsymbol{r})\right]_{\mathbb{P}}, \\
\Delta \mathcal{I}(\boldsymbol{r})= & \sum_{n=2}^{\infty} \int_{\mu_{f}^{2}}^{\mu_{\mathrm{H}}^{2}} \frac{d \mu^{2}}{\mu^{2}}\left[\frac{\alpha_{s}\left(\kappa_{\mathrm{R}} \mu^{2}\right)}{2 \pi}\right]^{n} \Delta \mathcal{S}_{\mathcal{Y}}^{(n)}\left(\mu^{2} ; \boldsymbol{r}\right) .
\end{aligned}
$$

If we use just $\mathcal{I}_{0}(\boldsymbol{r})$, we put just one shower splitting $\mathcal{S}$ into the exponent. This is the candidate for the summation of logarithms $L(\boldsymbol{r})$ as given by the shower. Its lowest order contribution, proportional to $\alpha_{s}\left(\mu_{\mathrm{H}}^{2}\right)$, will normally contain two powers of $L$ after integrating over $\mu^{2}$. One power comes from integrating over a momentum fraction $z$ inside $\mathcal{S}_{\mathcal{Y}}^{(1)}\left(\mu^{2} ; \boldsymbol{r}\right)$ and the second power comes from integrating over $\mu^{2}$. Thus we have a LL contribution $\alpha_{s}^{1} L^{2}$. We also generate terms with higher powers of $\alpha_{s}\left(\mu_{\mathrm{H}}^{2}\right)$, both from expanding the factor $\alpha_{s}\left(\kappa_{\mathrm{R}} \mu^{2}\right)$ inside the integral and from using $\mathcal{S}^{(n)}\left(\mu^{2}\right)$ for $n>1$. With appropriate choices for the algorithm that constitutes $\mathcal{S}\left(\mu^{2}\right)$, one may be able to generate a whole series of terms $\alpha_{s}^{n}\left(\mu_{\mathrm{H}}^{2}\right) L^{n+1}$ and $\alpha_{s}^{n}\left(\mu_{\mathrm{H}}^{2}\right) L^{n}$ that match a known QCD result at the LL and NLL levels.

Suppose that $\mathcal{I}_{0}(\boldsymbol{r})$ gives the expected QCD result for the summation of logarithms at the NLL level. What, then, does the complete shower algorithm give? For this, we must examine $\Delta \mathcal{I}(\boldsymbol{r})$. We need to ask whether $\Delta \mathcal{S}_{\mathcal{Y}}^{(n)}\left(\mu^{2} ; \boldsymbol{r}\right)$ is sufficiently small that it does not ruin the result from $\mathcal{I}_{0}(\boldsymbol{r})$. If $\Delta \mathcal{I}(\boldsymbol{r})$ contains no nonzero contributions proportional to $\alpha_{s}^{N}\left(\mu_{\mathrm{H}}^{2}\right) L^{j}(\boldsymbol{r})$ with $j>N+1$, then the logarithms $L$ exponentiate. If there are no nonzero contributions with $j \geq N+1$, then the shower sums the logarithms at the LL level. If there are no nonzero contributions with $j \geq N$, then the shower sums the logarithms at the NLL level.

Equations (96) and (97) provide a way to check how accurately the parton shower algorithm sums the large logarithms $L(\boldsymbol{r})$. Suppose that we wish to check whether the shower sums the logarithms at NLL accuracy. The best method is to prove analytically that $\Delta \mathcal{I}(\boldsymbol{r})$ meets the requirement for log summation at NLL accuracy. A second approach is to calculate the perturbative terms in $\Delta \mathcal{I}(\boldsymbol{r})$ as numerical integrals and check how many powers of $L(\boldsymbol{r})$ they contain. Although one can never check every term in $\Delta \mathcal{I}(\boldsymbol{r})$, this method has the advantage that if the check for NLL summation fails for any one contribution, then we know that NLL summation fails.

\section{REMARKS ABOUT THE GENERAL ANALYSIS}

It is, we think, of some importance to understand how accurately a parton shower algorithm sums large logarithms in an observable $\hat{\sigma}_{J}(v)$.

In analytical approaches to summing such logarithms, one typically defines an integral transform of the original distribution so that one considers a cross section $\sigma_{J}(\boldsymbol{r})$ that depends on parameters $\boldsymbol{r}$. Then the perturbative expansion of $\sigma_{J}(\boldsymbol{r})$ contains large logarithms $L(\boldsymbol{r})$.

Sometimes, one can compare the results of the shower for $\sigma_{J}(\boldsymbol{r})$ to the results in full QCD by writing the same differential equations as for full QCD but applying the differential operators to the shower approximation rather than full QCD [10,12]. This method has the disadvantage that one needs a separate and quite elaborate analysis for each observable to be studied.

An alternative is to calculate the observable $\hat{\sigma}_{J}(\boldsymbol{v})$ numerically with the parton shower event generator of interest and to compare the result with a known QCD result $[2,16]$. This method can work, at least for electron positron annihilation, but presents significant numerical challenges.

We have presented a reformulation of the calculation of $\sigma_{J}(\boldsymbol{r})$ according to a parton shower so that the large logarithms appear directly as an exponential. The exponent can be expanded perturbatively. This gives us a path to an analytical understanding the summation of these logarithms in the parton shower. It also provides a simple way to test this summation numerically.

In the sections that follow, we find interesting results for the thrust distribution in electron-positron annihilation. The analysis for electron-positron annihilation, we represent $\mathcal{I}(\boldsymbol{r})$ in a form that is somewhat less general than the form presented above but is better adapted to practical applications. Then we analyze $\mathcal{I}(\boldsymbol{r})$ analytically and numerically for the trust distribution in electron-positron annihilation.

\section{ANALYSIS FOR ELECTRON-POSITRON ANNIHILATION}

As outlined in the previous sections, a parton shower event generator can provide a QCD based approximation for a cross section $\hat{\sigma}_{J}(\boldsymbol{v})$ for an observable $J$ to take a value $\boldsymbol{v}$ in hadron-hadron, lepton-hadron, or electron-positron collisions. In the following sections, we concentrate on electron-positron annihilation, which is simpler because parton distribution functions do not appear. We begin in this section by framing the issues in a little more detail than we presented in Sec. I.

We suppose that the observable $J$ is infrared safe with a scale $\hat{Q}_{J}^{2}(\boldsymbol{v})$ substantially greater than $1 \mathrm{GeV}^{2}$. Then we can, at least in principle, omit a model for hadronization in the event generator. The QCD perturbative expansion for $\hat{\sigma}_{J}(\boldsymbol{v})$ will contain $\log$ arithms, $L=\log \left(\mu_{\mathrm{H}}^{2} / \hat{Q}_{J}^{2}(\boldsymbol{v})\right)$, where $\mu_{\mathrm{H}}^{2}$ is the scale of the hardest interaction in the event. Typically one finds perturbative contributions to $\hat{\sigma}_{J}(\boldsymbol{v})$ proportional to $\alpha_{s}^{n}\left(\mu_{\mathrm{H}}^{2}\right) L^{2 n}$. If $1 \mathrm{GeV}^{2} \ll \hat{Q}_{J}^{2}(\boldsymbol{v}) \ll \mu_{\mathrm{H}}^{2}$, and $L^{2} \gtrsim 1 / \alpha_{s}\left(\mu_{\mathrm{H}}^{2}\right)$, one must try to sum the contributions at each order of perturbation theory that have the most powers of $L$.

For some observables $J$ one can derive an analytical approximation, $\hat{\sigma}_{J}(\boldsymbol{v}$; analytical $)$, to $\hat{\sigma}_{J}(\boldsymbol{v})$ that sums the large logarithms in an appropriate sense. It is then of 
interest to see whether the parton shower generator sums the large logarithms at a specified level of approximation.

Normally, the approximation $\hat{\sigma}_{J}(\boldsymbol{v}$; shower $)$ obtained with a parton shower is limited to a numerical result obtained by averaging over many generated events. In the limit of very large hard scattering scales $\mu_{\mathrm{H}}^{2}$, $\hat{\sigma}_{J}(\boldsymbol{v}$; shower $)$ should match $\hat{\sigma}_{J}(\boldsymbol{v}$; analytical $)$. However, for $\mu_{\mathrm{H}}^{2}$ in the kinematic range of experiments, $\hat{\sigma}_{J}(\boldsymbol{v}$; shower $)$ contains effects that are numerically important but are not included in $\hat{\sigma}_{J}(\boldsymbol{v}$; analytical $)$. Thus it is difficult to tell whether $\hat{\sigma}_{J}(\boldsymbol{v}$; shower $)$ agrees with $\hat{\sigma}_{J}(\boldsymbol{v}$; analytical $)$.

One approach to comparing $\hat{\sigma}_{J}(\boldsymbol{v}$; shower $)$ to $\hat{\sigma}_{J}(\boldsymbol{v}$; analytical $)$ is to directly calculate $\hat{\sigma}_{J}(\boldsymbol{v}$; shower $)$ for a sequence of very large hard scattering scales $\mu_{\mathrm{H}}^{2}$ that are far from the range of experiments. This approach can work [16], and in fact we use it to a limited extent in this paper. However, it is difficult to maintain the required numerical accuracy at very large values of $\mu_{\mathrm{H}}^{2}$ in a practical parton shower event generator.

In an analytical approach, one typically starts by taking an appropriate integral transform of $\hat{\sigma}_{J}(\boldsymbol{v})$. Then one calculates a cross section $\sigma_{J}(\boldsymbol{r})$ depending on a variable or variables $\boldsymbol{r}$. For instance, one may be interested in the distribution of the thrust parameter, $T$, so that one examines $\hat{\sigma}_{J}(\tau)$ where $\tau=1-T$. Then one takes the Laplace transform of $\hat{\sigma}_{J}(\tau)$ with Laplace parameter $\nu$. Then we need to sum logarithms $L=\log (\nu)$, which is large when $\nu \rightarrow \infty$.

The aim of the following sections is to follow the general method outlined in Secs. II through X so as to redesign the calculation of the parton shower cross section so that it produces the same result for the integral transform of the cross section as before but so that it produces a calculation of this quantity and not a cross section for other observables. The redesigned calculation gives the integral transform of interest as an exponential of a quantity that can be expanded in powers of the shower splitting operator. The leading order term in the exponent is simple and is the candidate for the summation of large logarithms produced by the shower. If the higher order contributions to the exponent are suitably small, then they to not interfere with the summation represented by the leading order terms. In some favorable cases, we can analyze all higher order contributions to the exponent analytically. In other cases, we calculate low order contributions to the exponent numerically.

Our example is the thrust distribution. There is much to be learned from this example. In particular, we learn that the shower result depends on some details of the parton shower algorithm that one might have thought are not important.

\section{THE PARTON SHOWER FRAMEWORK}

We begin with a brief review of the parton shower framework that we will use, expanding on the material at the start of Sec. II, but simplifying this material with respect to initial state partons, which do not appear in electronpositron annihilation, and with respect to spin.

A parton shower can be described using operators on a vector space, the "statistical space," that describes the momenta, flavors, colors, and spins for all of the partons created in a shower as the shower develops. We use this description in the parton shower event generator DEDUCTOR [18-25,31,32]. The general theory includes parton spins but DEDUCTOR simply averages over spins, so our explanation in the following sections will leave out parton spins. With $m$ final state partons in electron-positron annihilation, the partons carry labels $1,2, \ldots, m$. The partons have momenta $\{p\}_{m}=\left\{p_{1}, \ldots, p_{m}\right\}$ and flavors $\{f\}_{m}$. We take the partons to be massless: $p_{i}^{2}=0$. For color, there are ket color basis states $\left|\{c\}_{m}\right\rangle$ and bra color basis states $\left\langle\left\{c^{\prime}\right\}_{m}\right|$. We use the trace basis (or color-string basis), as described in Sec. 7 of Ref. [19]. Color appears in the statistical space as the density matrix, with basis elements $\left|\{c\}_{m}\right\rangle\left\langle\left\{c^{\prime}\right\}_{m}\right|$. Then the $m$-parton basis states for the statistical space are denoted by $\left.\mid\left\{p, f, c, c^{\prime}\right\}_{m}\right)$. The statistical space is described in Secs. 2 and 3 of Ref. [19]. These sections also show how shower evolution is expressed using evolution operators that act on the statistical space. (However, in this paper the names of the operators follow Ref. [18] rather than Ref. [19].)

Parton shower programs often use the leading color (LC) approximation, which provides the leading term in an expansion in powers of $1 / N_{c}^{2}=1 / 9[4,27,28]$. With this approach, the color states $\left\{c, c^{\prime}\right\}_{m}$ obey $\left\{c^{\prime}\right\}_{m}=\{c\}_{m}$. The splitting probabilities are simply proportional to a factor $C_{\mathrm{F}}$ or $C_{\mathrm{A}}$, with $C_{\mathrm{F}}$ being equivalent to $C_{\mathrm{A}} / 2$ within the $\mathrm{LC}$ approximation.

Our program, Deductor [23], uses what is called the LC+ approximation [22]. The LC+ approximation consists of simply dropping some color operator contributions in the splitting functions. The $\mathrm{LC}+$ approximation is more powerful than the LC approximation because it has corrections only for soft, finite angle emissions but is exact in the limit of collinear emissions [22]. For this reason, the LC+ approximation is more accurate than the LC approximation for summing large logarithms correctly in a parton shower. Reference [17] analyzes the effect of various forms of the LC approximation on the summation of large logarithms.

In this paper, we focus on the effect on the summation of large logarithms from characteristics of the parton shower formulation such as the ordering variable, the momentum mapping, and the splitting functions. We do not focus on the treatment of color. Thus we mostly use full QCD color without approximation. Where we simply run DEDUCTOR to produce the thrust distribution, we use DEDUCTOR's default color approximation, LC+. There, for the DEDUCTOR default ordering variable and momentum mapping, we verify numerically that the $\mathrm{LC}+$ approximation is essentially exact for the thrust distribution. (See Fig. 4.) At one point, in Fig. 5, we make contact with Ref. [17] by 
investigating what happens when one uses a less exact color approximation that is one version of the LC approximation.

DEDUCTOR uses specific choices with respect to shower kinematics, the shower ordering variable, and the parton splitting functions. In the remainder of this section, we outline some of these choices that play a role in the analysis of the following sections.

In DEDUCTOR, the default is to order splittings according to decreasing values of a hardness parameter $\Lambda^{2}$ [24]. This hardness parameter is based on virtuality. For massless final state partons in electron-positron collisions, the definition is ${ }^{4}$

$$
\Lambda^{2}=\frac{\left(\hat{p}_{l}+\hat{p}_{m+1}\right)^{2}}{2 p_{l} \cdot Q} Q^{2} .
$$

Here the mother parton in a final state splitting has momentum $p_{l}$ and the daughters have momenta $\hat{p}_{l}$ and $\hat{p}_{m+1}$. Here $Q$ is the total momentum $Q$ of all of the final state partons, which remains the same throughout the shower. It proves convenient to use a dimensionless virtuality variable $y=\Lambda^{2} / Q^{2}$ :

$$
y=\frac{\left(\hat{p}_{l}+\hat{p}_{m+1}\right)^{2}}{2 p_{l} \cdot Q} .
$$

Thus $y$ decreases from one shower splitting to the next.

One could use a hardness parameter other than $\Lambda$ to order the shower. We will consider also a shower ordered by the transverse momentum [25] in a splitting,

$$
k_{\mathrm{T}}^{2}=z(1-z)\left(\hat{p}_{l}+\hat{p}_{m+1}\right)^{2}=z(1-z) y Q^{2} / a_{l},
$$

where $z$ is the momentum fraction in the splitting and

$$
a_{l}=\frac{Q^{2}}{2 p_{l} \cdot Q} .
$$

We denote the hardness scale of a splitting by $\mu^{2}$. When we use the default ordering variable $\Lambda$ for the shower, then $\mu^{2}=\Lambda^{2}$. If we use $k_{\mathrm{T}}$ ordering, then $\mu^{2}=k_{\mathrm{T}}^{2}$.

To measure an infrared-safe observable $\mathcal{O}_{J}$ in electronpositron annihilation, we can use the notation

$$
\sigma_{J}=\left(1\left|\mathcal{O}_{J} \mathcal{U}\left(\mu_{f}^{2}, Q^{2}\right)\right| \rho_{\mathrm{H}}\right) .
$$

Here $\left(\rho_{\mathrm{H}}\right)$ is the starting parton state for the hard scattering process. If we were to evaluate $\left.\mid \rho_{\mathrm{H}}\right)$ beyond leading order, then it would contain appropriate subtractions to remove infrared singularities. In this paper, we evaluate $\left.\mid \rho_{\mathrm{H}}\right)$ at lowest order so that it is simply a $q \bar{q}$ state. We associate a scale $\mu_{\mathrm{H}}^{2}=Q^{2}$ with the hard scattering, where $Q$ is the $q \bar{q}$

\footnotetext{
${ }^{4}$ In hadron-hadron collisions, $Q$ in Eq. (98) is replaced by the momentum $Q_{0}$ of the final state partons at the start of the shower.
}

momentum. The operator $\mathcal{U}\left(\mu_{f}^{2}, Q^{2}\right)$ expresses the evolution of the system from the scale $Q^{2}$ to a scale $\mu_{f}^{2}$ of order $1 \mathrm{GeV}^{2}$, at which the shower is turned off. After this evolution, we have a statistical state that can be expanded in the basis states $\left.\mid\left\{p, f, c, c^{\prime}\right\}_{m}\right)$. This expansion is realized as an integral, which takes the form of a Monte Carlo integration that is obtained by generating many Monte Carlo events. We then apply an operator $\mathcal{O}_{J}$ that embodies the desired measurement. We still have a sum and integral of basis states. We take the product with the statistical bra state (1|, which is defined by

$$
\left(1 \mid\left\{p, f, c, c^{\prime}\right\}_{m}\right)=\left\langle\left\{c^{\prime}\right\}_{m} \mid\{c\}_{m}\right\rangle .
$$

This leaves us with the numerical result for $\sigma_{J}$. The use of the statistical bra vector (1) is discussed in Sec. 3.5 of Ref. [19].

The shower operator $\mathcal{U}$ takes the form

$$
\mathcal{U}\left(\mu_{2}^{2}, \mu_{1}^{2}\right)=\mathbb{T} \exp \left(\int_{\mu_{2}^{2}}^{\mu_{1}^{2}} \frac{d \mu^{2}}{\mu^{2}} \mathcal{S}\left(\mu^{2}\right)\right) .
$$

There is an instruction $\mathbb{t}$ that indicates that if we expand the exponential, the operators $\mathcal{S}\left(\mu^{2}\right)$ with the smallest values of $\mu^{2}$ belong on the left. This is simply a compact way of saying that $\mathcal{U}\left(\mu_{2}^{2}, \mu_{1}^{2}\right)$ obeys the differential equation

$$
\mu_{1}^{2} \frac{\partial}{\partial \mu_{1}^{2}} \mathcal{U}\left(\mu_{2}^{2}, \mu_{1}^{2}\right)=\mathcal{U}\left(\mu_{2}^{2}, \mu_{1}^{2}\right) \mathcal{S}\left(\mu_{1}^{2}\right)
$$

In general, the generator $\mathcal{S}\left(\mu^{2}\right)$ is a sum of terms with approximations to $n_{\mathrm{R}}$ real emissions and $n_{\mathrm{V}}$ virtual exchanges,

$$
\mathcal{S}\left(\mu^{2}\right)=\sum_{\substack{n_{\mathrm{R}}, n_{\mathrm{V}}=0 \\ n_{\mathrm{R}}+v_{\mathrm{V}} \geq 1}}^{\infty} \mathcal{S}^{\left[n_{\mathrm{R}}, n_{\mathrm{V}}\right]}\left(\mu^{2}\right) .
$$

In existing parton shower event generators like DEDUCTOR, only the terms with $n_{\mathrm{R}}+n_{\mathrm{V}}=1$ are implemented. This is also the case for other parton shower algorithms that we consider here. Thus we assume

$$
\mathcal{S}\left(\mu^{2}\right)=\mathcal{S}^{[1,0]}\left(\mu^{2}\right)+\mathcal{S}^{[0,1]}\left(\mu^{2}\right) .
$$

The operator $\mathcal{S}^{[1,0]}\left(\mu^{2}\right)$ creates a splitting, changing an $m$ parton state to an $m+1$ parton state. The operator $\mathcal{S}^{[0,1]}\left(\mu^{2}\right)$ leaves the number of partons and their momenta and flavors unchanged, although in a full color treatment it modifies the parton color state. The operator $\mathcal{S}^{[0,1]}\left(\mu^{2}\right)$ is related to the inclusive sum over splitting variables in $\mathcal{S}^{[1,0]}\left(\mu^{2}\right)$ by $\left(1 \mid \mathcal{S}^{[0,1]}\left(\mu^{2}\right)=-\left(1 \mid \mathcal{S}^{[1,0]}\left(\mu^{2}\right)\right.\right.$, so that

$$
\left(1 \mid \mathcal{S}\left(\mu^{2}\right)=0\right. \text {. }
$$


If we had contributions to the shower generator with $n_{\mathrm{R}}+n_{\mathrm{V}}>1$, we would still have $\left(1 \mid \mathcal{S}\left(\mu^{2}\right)=0\right.$ [18].

The operator $\mathcal{S}^{[1,0]}\left(y Q^{2}\right)$ in DedUCTOR [22,25] is not simple. However, in the cases for which we need an explicit expression in our analytical formulas here, we need only its form when $y \ll 1$ and $(1-z) \ll 1$. This is the limit in which $\mathcal{S}^{[1,0]}\left(y Q^{2}\right)$ expresses the soft $\times$ collinear double singularity of QCD. (However, our numerical results use the full $\mathcal{S}^{[1,0]}\left(y Q^{2}\right)$.) In this limit, we have

$$
\begin{aligned}
& \mathcal{S}^{[1,0]}(\left.\left(y Q^{2}\right) \mid\left\{p, f, c, c^{\prime}\right\}_{m}\right) \\
& \approx\left.-\sum_{l=1}^{m} \sum_{\substack{k=1 \\
k \neq l}}^{m}\left[T_{l} \otimes T_{k}^{\dagger}+T_{k} \otimes T_{l}^{\dagger}\right] \mid\left\{c, c^{\prime}\right\}_{m}\right) \\
& \quad \times \int \frac{d \phi}{2 \pi} \int \frac{d z}{1-z} \frac{\alpha_{s}\left(\lambda_{\mathrm{R}}(1-z) y Q^{2} / a_{l}\right)}{2 \pi} \\
&\left.\quad \times \Theta\left(\frac{a_{l} y}{\vartheta(l, k)}<1-z<1\right) \mid\{\hat{p}, \hat{f}\}_{m+1}\right) .
\end{aligned}
$$

There is a sum over parton indices $l$ and $k$. We split parton $l$ with dipole partner parton $k$, creating a new parton $m+1$, which we consider to be a gluon. The momenta $\{\hat{p}\}_{m+1}$ of the partons after the splitting are functions of the momenta $\{p\}_{m}$ before the splitting and the splitting variables $y, z, \phi$, as specified in Eqs. (189) and (194).

In Eq. (109), $\left[T_{l} \otimes T_{k}^{\dagger}\right]$ and $\left[T_{k} \otimes T_{l}^{\dagger}\right]$ are operators on the parton color space. The notation $\left(C_{\mathrm{ket}} \otimes C_{\mathrm{bra}}^{\dagger}\right)$ for color operators represents the following. A color basis vector $\left.\mid\left\{c, c^{\prime}\right\}_{m}\right)$ in the statistical space represents the color density operator $\left|\{c\}_{m}\right\rangle\left\langle\left\{c^{\prime}\right\}_{m}\right|$. Here $\left|\{c\}_{m}\right\rangle$ and $\left|\left\{c^{\prime}\right\}_{m}\right\rangle$ are basis vectors for color amplitudes. Let $C_{\text {ket }}$ and $C_{\text {bra }}$ be operators on color amplitudes for $m$ partons that yield color amplitudes for $\hat{m}$ partons with $\hat{m} \geq m$. In the case of $\mathcal{S}^{[1,0]}\left(y Q^{2}\right), \quad \hat{m}=m+1$. The statistical space vector $\left.\left(C_{\text {ket }} \otimes C_{\text {bra }}^{\dagger}\right) \mid\left\{c, c^{\prime}\right\}_{m}\right)$ then represents the color density operator $C_{\text {ket }}\left|\{c\}_{m}\right\rangle\left\langle\left\{c^{\prime}\right\}_{m}\right| C_{\text {bra }}^{\dagger}$. In the case of $\left[T_{l} \otimes T_{k}^{\dagger}\right]$, the operator creates a new gluon with color index $a$ by inserting a color generator matrix $T^{a}$ on the color line for parton $l$ in the ket state and inserting $T^{a}$ on the color line for parton $k$ in the bra state.

The argument of $\alpha_{\mathrm{s}}$ in Eq. (109) contains the standard factor [7]

$$
\lambda_{\mathrm{R}}=\exp \left(-\frac{C_{\mathrm{A}}\left(67-3 \pi^{2}\right)-10 n_{f}}{3\left(11 C_{\mathrm{A}}-2 n_{f}\right)}\right) .
$$

The rest of the argument of $\alpha_{\mathrm{S}}$ is $k_{\mathrm{T}}^{2}$, Eq. (100), except that we drop the factor $z$ because we are interested only in small $1-z$. Although the operators $\mathcal{S}^{[1,0]}\left(\mu^{2}\right)$ contain one power of $\alpha_{s}$, this $\alpha_{s}$ is evaluated at a scale that is not $\mu^{2}$. Thus if we expand $\mathcal{S}\left(\mu^{2}\right)$ in powers of $\alpha_{s}\left(\mu^{2}\right)$, all powers will appear.
The parameter $\vartheta(l, k)$ is

$$
\vartheta(l, k)=\frac{1}{2}[1-\cos (\theta(l, k))],
$$

where $\theta(l, k)$ is the angle between partons $l$ and $k$ in $\left.\mid\left\{p, f, c, c^{\prime}\right\}_{m}\right)$. With this definition, $\vartheta \approx \theta^{2} / 4$ for small $\theta$. The angle $\hat{\theta}(l, m+1)$ between partons $l$ and $m+1$ after a splitting is given by

$$
\begin{aligned}
1-\cos (\hat{\theta}(l, m+1)) & =\frac{Q^{2}}{\hat{p}_{l} \cdot Q \hat{p}_{m+1} \cdot Q} \hat{p}_{l} \cdot \hat{p}_{m+1} \\
& \sim \frac{2 a_{l} y}{z(1-z)}+\mathcal{O}\left(y^{2}\right) .
\end{aligned}
$$

For small $y$ and small $(1-z)$, this gives

$$
\hat{\vartheta}(l, m+1) \approx \frac{a_{l} y}{1-z} .
$$

Thus the lower limit on $(1-z)$ is equivalent to an upper limit on the splitting angle, $\hat{\vartheta}(l, m+1)<\vartheta(l, k)$. The splitting angle should be smaller than the angle between the two partons $l$ and $k$. The restriction $(1-z)<1$ gives a lower limit on the splitting angle. The net range for the new splitting angle is

$$
a_{l} y<\hat{\vartheta}(l, m+1)<\vartheta(l, k) .
$$

\section{PREVIEW}

In the following sections, we propose a way, for electronpositron annihilation, to gain more direct access to the summation of large logarithms in a parton shower than by simply running the shower and examining the result numerically. The analysis adapts the general formulation of the method in Secs. II through X to the practical analysis of first order parton shower algorithms. Our example is the thrust distribution in electron-positron annihilation. Here is a brief preview.

(i) We are interested in the thrust distribution $g(\tau)$ with $\tau=1-T$, where $T$ is the thrust.

(ii) As in analytical approaches, we work with the Laplace transform $\tilde{g}(\nu)$ of $g(\tau)$.

(iii) $\tilde{g}(\nu)$ contains large logarithms, $\alpha_{s}\left(\mu_{\mathrm{H}}^{2}\right)^{n} \log ^{j}(\nu)$ with $j \leq 2 n$.

(iv) We suppose that we know the proper summation of the $\log (\nu)$ factors in full QCD at a certain level of accuracy, but a leading order parton shower is not full QCD. We wish to know what result the parton shower gives.

(v) The result of simply running the shower and examining the result numerically can be expressed as in Eq. (102), 


$$
\tilde{g}(\nu)=\frac{1}{\sigma_{\mathrm{H}}}\left(1\left|\mathcal{O}(\nu) \mathcal{U}\left(\mu_{f}^{2}, Q^{2}\right)\right| \rho_{\mathrm{H}}\right)
$$

Here $\sigma_{\mathrm{H}}$ is the total hard scattering cross section and $\left(1|\cdots| \rho_{\mathrm{H}}\right)$ indicates an ensemble average in the statistical state $\left(\rho_{\mathrm{H}}\right)$ representing the perturbative hard scattering. Then $\mathcal{U}\left(\mu_{f}^{2}, Q^{2}\right)$ represents the operator on the statistical space that generates the shower. This gives us states consisting of tens of partons. We could measure any operator $\mathcal{O}_{J}$ that we like in this many-parton state. We apply a simple operator $\mathcal{O}(\nu)$ that measures the Laplace transformed thrust distribution on this state.

(vi) In this paper, we rewrite $\tilde{g}(\nu)$ in the form

$$
\tilde{g}(\nu)=\frac{1}{\sigma_{\mathrm{H}}}\left(1|\mathbb{T} \exp (\mathcal{I}(\nu)) \mathcal{O}(\nu)| \rho_{\mathrm{H}}\right) .
$$

The notation $\mathbb{T}$ indicates an ordering instruction for the exponential, as in Eq. (104) and later in Eq. (145). In the example used in this paper, the operator $\mathcal{O}(\nu)$ applied to the hard state $\left.\mid \rho_{\mathrm{H}}\right)$ simply gives an eigenvalue 1 .

(vii) With this form, we have expressed $\tilde{g}(\nu)$ in terms of the exponential of an operator $\mathcal{I}(\nu)$. This operator has an expansion

$$
\mathcal{I}(\nu)=\sum_{k=1}^{\infty} \mathcal{I}^{[k]}(\nu)
$$

where each term in $\mathcal{I}^{[k]}(\nu)$ contains $k$ factors of the splitting operator $\mathcal{S}$.

(viii) We can further expand in powers of $\alpha_{s}$ evaluated at a fixed scale $Q^{2} / \nu$ :

$$
\mathcal{I}^{[k]}(\nu)=\sum_{n=k}^{\infty}\left[\frac{\alpha_{s}\left(Q^{2} / \nu\right)}{2 \pi}\right]^{n} \mathcal{I}_{n}^{[k]}(\nu) .
$$

(ix) The most important feature of Eq. (116) is that the operators $\mathcal{I}^{[k]}(\nu)$ can be computed using two fairly simple recursion relations.

(x) The first order contribution, $\mathcal{I}^{[1]}(\nu)$, is obtained rather trivially from one power of the shower splitting operator $\mathcal{S}\left(\mu^{2}\right)$. This operator is then the obvious candidate for the exponentiation of $\tilde{g}(\nu)$ generated by the shower. If $\mathcal{S}\left(\mu^{2}\right)$ is suitably defined, $\mathcal{I}^{[1]}(\nu)$ matches the exponentiation in full QCD.

\footnotetext{
${ }^{5}$ In Secs. II through X, we expanded operators in powers of $\alpha_{s}\left(\mu^{2}\right)$ at a running scale $\mu^{2}$ appropriate to the operator. Here, we expand operators in powers of the splitting operator $\mathcal{S}$ of the parton shower. This technique simplifies the analysis of a shower algorithm that is based on lowest order perturbation theory.
}

(xi) If $\mathcal{I}^{[1]}(\nu)$ generates the desired exponentiation, then $\mathcal{I}^{[k]}(\nu)$ for $k \geq 2$ should be small, so as not to destroy the desired exponentiation.

(xii) For next-to-leading-log summation (NLL), this implies that $\mathcal{I}_{n}^{[k]}(\nu)$ should not contain more than $n-1$ powers of $\log (\nu)$.

(xiii) In one case examined in this paper, we can show analytically that $\mathcal{I}_{n}^{[k]}(\nu)$ does not contain more than $n-1$ powers of $\log (\nu)$.

(xiv) The operator $\mathcal{I}_{2}^{[2]}(\nu)$ is of special interest. It should not contain more than one power of $\log (\nu)$.

(xv) In some cases, we can show analytically that $\mathcal{I}_{2}^{[2]}(\nu)$ does not contain more than one power of $\log (\nu)$.

(xvi) We can write the integral for $\mathcal{I}_{n}^{[2]}(\nu)$ and evaluate it numerically to see if it contains more than $n-1$ powers of $\log (\nu)$.

(xvii) For some shower algorithms examined here, $\mathcal{I}_{n}^{[2]}(\nu)$ passes this test. For one algorithm examined, it fails.

\section{THE THRUST DISTRIBUTION AND ITS LAPLACE TRANSFORM}

We will examine the distribution of thrust, $T$, defined for parton momenta $\{p\}_{m}$ by $[33,34]$

$$
T=\max _{\vec{n}_{\mathrm{T}}} \frac{\sum_{i}\left|\vec{p}_{i} \cdot \vec{n}_{\mathrm{T}}\right|}{\sum_{i}\left|\vec{p}_{i}\right|}=\frac{1}{\sqrt{Q^{2}}} \max _{\vec{n}_{\mathrm{T}}} \sum_{i}\left|\vec{p}_{i} \cdot \vec{n}_{\mathrm{T}}\right| .
$$

The axis defined by the unit vector $\vec{n}_{\mathrm{T}}$ that maximizes the sum is the thrust axis. We will be interested in the behavior of the thrust distribution for small values of

$$
\tau=1-T \text {. }
$$

We can write $\tau$ in a useful form by defining sets $R$ and $L$ of partons by $\vec{p}_{i} \cdot \vec{n}_{\mathrm{T}}>0$ for $i \in R$ and $\vec{p}_{i} \cdot \vec{n}_{\mathrm{T}}<0$ for $i \in L$. Then,

$$
\tau=\frac{1}{\sqrt{Q^{2}}}\left[\sum_{i \in R}\left(E_{i}-\vec{p}_{i} \cdot \vec{n}_{\mathrm{T}}\right)+\sum_{i \in L}\left(E_{i}+\vec{p}_{i} \cdot \vec{n}_{\mathrm{T}}\right)\right] .
$$

Using the thrust axis, we define \pm components of vectors by

$$
p^{ \pm}=\left[p_{i}^{0} \pm \vec{p} \cdot \vec{n}_{\mathrm{T}}\right] / \sqrt{2}
$$

Then we can write

$$
\tau=\tau_{R}+\tau_{L}
$$

where, using $Q^{2}=2 Q^{+} Q^{-}$with $Q^{+}=Q^{-}$, 


$$
\tau_{R}=\sum_{i \in R} \frac{p_{i}^{-}}{Q^{-}}, \quad \tau_{L}=\sum_{i \in L} \frac{p_{i}^{+}}{Q^{+}}
$$

In order to use a parton shower to analyze the thrust distribution, we begin with the cross section

$$
g(\tau)=\frac{1}{\sigma_{\mathrm{H}}} \frac{d \sigma}{d \tau}
$$

where $\sigma_{\mathrm{H}}$ is the hard scattering cross section, equal to $d \sigma / d \tau$ integrated over $\tau$. We wish to analyze the small $\tau$ behavior of $g(\tau)$. For this purpose, it is standard to work with the Laplace transform of $g(\tau)$,

$$
\tilde{g}(\nu)=\int_{0}^{\infty} d \tau e^{-\nu \tau} g(\tau)
$$

The coefficient of $\alpha_{s}^{n}$ in the perturbative expansion of $g(\tau)$ is not a normal function but is a distribution with $\log ^{j-1}(\tau) / \tau$ singularities at $\tau=0$. In order to work with normal functions, we define the integral of $g(\tau)$,

$$
f(\tau)=\int_{0}^{\tau} d \bar{\tau} g(\bar{\tau})
$$

The coefficients in the perturbative expansion of $f(\tau)$ are functions with $\log ^{j}(\tau)$ integrable singularities. The cross section $g(\bar{\tau})$ vanishes for $\bar{\tau}>1 / 2$, so $f(\tau)=1$ for $\tau>1 / 2$.

Consider the Laplace transform of $f(\tau)$ :

$$
\tilde{f}(\nu)=\int_{0}^{\infty} d \tau e^{-\nu \tau} f(\tau) .
$$

We have

$$
\begin{aligned}
\tilde{f}(\nu) & =\int_{0}^{\infty} d \tau^{\prime} e^{-\nu \tau^{\prime}} \int_{0}^{\tau^{\prime}} d \tau g(\tau) \\
& =\int_{0}^{\infty} d \tau g(\tau) \int_{\tau}^{\infty} d \tau^{\prime} e^{-\nu \tau^{\prime}} \\
& =\frac{1}{\nu} \int_{0}^{\infty} d \tau g(\tau) e^{-\nu \tau} .
\end{aligned}
$$

Thus

$$
\tilde{f}(\nu)=\frac{\tilde{g}(\nu)}{\nu} .
$$

The function $f(\tau)$ is given by the inverse Laplace transform of $\tilde{f}(\nu)$ :

$$
f(\tau)=\frac{1}{2 \pi \mathrm{i}} \int_{C} d \nu e^{\nu \tau} \frac{\tilde{g}(\nu)}{\nu} .
$$

The contour $C$ runs from $\nu_{0}-\mathrm{i} \infty$ to $\nu_{0}+\mathrm{i} \infty$ parallel to the imaginary $\nu$ axis, where $\nu_{0}>0$ so that the contour is to the right of the singularity of $\tilde{g}(\nu) / \nu$ at $\nu=0$.

We expect the coefficient of $\alpha_{s}^{n}$ in the perturbative expansion of $f(\tau)$ to contain terms proportional to $\log ^{j}(\tau)$ for $\tau \rightarrow 0$. To see how this translates to $\tilde{g}(\nu)$, we can start by noting that

$$
f(\tau)=\tau^{A} \Rightarrow \tilde{g}(\nu)=\Gamma(1+A) \nu^{-A} .
$$

Thus

$$
\begin{aligned}
& f(\tau)=\sum_{j=0}^{\infty} \frac{A^{j}}{j !} \log ^{j}(\tau) \Rightarrow \\
& \tilde{g}(\nu)=\Gamma(1+A) \sum_{j=0}^{\infty} \frac{(-A)^{j}}{j !} \log ^{j}(\nu) .
\end{aligned}
$$

Matching powers of $A$, we learn that logarithms of $\tau$ for small $\tau$ translate into logarithms of $\nu$ for large $\nu$.

We wish to use the parton shower formalism to find an analytical formula that sums the logarithms of $\nu$ in $\tilde{g}(\nu)$. We can then compare what we find to the standard QCD formula that sums these logarithms. The final step needed to obtain something that can be compared to experiment would be to perform the inverse Laplace transform (131). This step is the same for the parton shower method or the normal analytical methods. We discuss this step only briefly in this paper.

\section{THE MEASUREMENT OPERATOR}

If we want to measure the thrust distribution, then we define, following Eq. (102),

$$
g(\tau)=\frac{1}{\sigma_{\mathrm{H}}}\left(1\left|\delta\left(\tau-\tau_{\mathrm{op}}\right) \mathcal{U}\left(\mu_{f}^{2}, \mu_{\mathrm{H}}^{2}\right)\right| \rho_{\mathrm{H}}\right),
$$

where $\tau$ is a real number times the unit operator on the statistical space and $\tau_{\mathrm{op}}$ is the operator defined by

$$
\left.\left.\tau_{\mathrm{op}} \mid\left\{p, f, c, c^{\prime}\right\}_{m}\right)=\tau\left(\{p\}_{m}\right) \mid\left\{p, f, c, c^{\prime}\right\}_{m}\right),
$$

where $\tau\left(\{p\}_{m}\right)$ is $1-T$ for partons with momenta $\{p\}_{m}$, as defined in Eqs. (123) and (124). Here $\sigma_{\mathrm{H}}=\left(1 \mid \rho_{\mathrm{H}}\right)$. This is the Born cross section for $e^{+} e^{-} \rightarrow q \bar{q}$ since, in this paper, we evaluate $\left.\mid \rho_{\mathrm{H}}\right)$ at lowest order.

Rather than measuring $g(\tau)$, we wish to measure the Laplace transform $\tilde{g}(\nu)$. For this we have, using Eq. (126) in Eq. (134),

$$
\tilde{g}(\nu)=\frac{1}{\sigma_{\mathrm{H}}}\left(1\left|\mathcal{O}(\nu) \mathcal{U}\left(\mu_{f}^{2}, Q^{2}\right)\right| \rho_{\mathrm{H}}\right),
$$

where 


$$
\mathcal{O}(\nu)=e^{-\nu \tau_{\mathrm{op}}} .
$$

We will analyze $\tilde{g}(\nu)$ in the subsequent sections. For this analysis, it is important that $\mathcal{O}(\nu)$ has an inverse

$$
\mathcal{O}(\nu)^{-1}=e^{\nu \tau_{\mathrm{op}}}
$$

\section{SETTING UP THE SHOWER ANALYSIS}

Equation (136) allows us to calculate $\tilde{g}(\nu)$ numerically using the shower evolution operator $\mathcal{U}\left(\mu_{f}^{2}, \mu_{\mathrm{H}}^{2}\right)$. We would now like to reformulate the shower result so that it takes the form of an exponential in which the exponent can be perturbatively calculated.

\section{A. The operators $\mathcal{Y}$ and $\mathcal{S}_{\mathcal{Y}}$}

We begin with an operator $\mathcal{Y}\left(\mu^{2} ; \nu\right)$, which is defined in Secs. III and VIII using the all-order formalism of Ref. [18] for describing parton shower algorithms. The operator $\mathcal{Y}\left(\mu^{2} ; \nu\right)$ is defined to have two properties. First, it does not change the number of partons or their momenta or flavors. Second,

$$
\left(1 \mid \mathcal{Y}\left(\mu^{2} ; \nu\right)=\left(1 \mid \mathcal{O}(\nu) \mathcal{U}\left(\mu_{f}^{2}, \mu^{2}\right) \mathcal{O}^{-1}(\nu) .\right.\right.
$$

These properties apply either for electron-positron, hadronhadron, or electron-hadron collisions. Now, we consider only electron-positron annihilation. Although $\mathcal{Y}\left(\mu^{2} ; \nu\right)$ does not change the number of partons or their momenta or flavors, it can change the parton colors. There is some freedom to define what $\mathcal{Y}\left(\mu^{2} ; \nu\right)$ does to the parton color state. We will define the action of $\mathcal{Y}\left(\mu^{2} ; \nu\right)$ on states in the statistical space in Eqs. (170) and (171) below.

The property Eq. (139) can be written as

$$
\left(1 \mid \mathcal{O}(\nu) \mathcal{U}\left(\mu_{\mathrm{f}}^{2}, \mu^{2}\right)=\left(1 \mid \mathcal{Y}\left(\mu^{2} ; \nu\right) \mathcal{O}(\nu) .\right.\right.
$$

This result allows us to rewrite $\tilde{g}(\nu)$ as given by Eq. (136) as

$$
\tilde{g}(\nu)=\frac{1}{\sigma_{\mathrm{H}}}\left(1\left|\mathcal{Y}\left(Q^{2} ; \nu\right) \mathcal{O}(\nu)\right| \rho_{\mathrm{H}}\right) .
$$

We see that instead of generating a complete parton shower as in Eq. (136) and then measuring $\mathcal{O}(\nu)$ for the resulting many parton state, we can measure $\mathcal{O}(\nu)$ just on the hard state and then apply the operator $\mathcal{Y}\left(\mu^{2} ; \nu\right)$ that depends on $\nu$ but leaves the number of partons unchanged.

How can one evaluate $\mathcal{Y}\left(\mu^{2} ; \nu\right)$ ? We note first from the form of Eq. (139), that $\mathcal{Y}\left(\mu^{2} ; \nu\right)$ has a perturbative expansion beginning with $\mathcal{Y}\left(\mu^{2} ; \nu\right)=1+\mathcal{O}\left(\alpha_{s}\right)$ and at $\mu^{2}=\mu_{f}^{2}$ it is exactly

$$
\mathcal{Y}\left(\mu_{f}^{2} ; \nu\right)=1
$$

We define an infinitesimal generator $\mathcal{S}_{\mathcal{Y}}\left(\mu^{2} ; \nu\right)$ for $\mathcal{Y}\left(\mu^{2} ; \nu\right)$ by

$$
\frac{1}{\mu^{2}} \mathcal{S}_{\mathcal{Y}}\left(\mu^{2} ; \nu\right)=\mathcal{Y}^{-1}\left(\mu^{2} ; \nu\right) \frac{d}{d \mu^{2}} \mathcal{Y}\left(\mu^{2} ; \nu\right) .
$$

Then $\mathcal{Y}\left(\mu^{2} ; \nu\right)$ obeys the differential equation

$$
\mu^{2} \frac{d}{d \mu^{2}} \mathcal{Y}\left(\mu^{2} ; \nu\right)=\mathcal{Y}\left(\mu^{2} ; \nu\right) \mathcal{S}_{\mathcal{Y}}\left(\mu^{2} ; \nu\right)
$$

with boundary condition $\mathcal{Y}\left(\mu_{f}^{2} ; \nu\right)=1$. We can use the notation

$$
\mathcal{Y}\left(\mu^{2} ; \nu\right)=\mathbb{T} \exp \left(\int_{\mu_{f}^{2}}^{\mu^{2}} \frac{d \bar{\mu}^{2}}{\bar{\mu}^{2}} \mathcal{S}_{\mathcal{Y}}\left(\bar{\mu}^{2} ; \nu\right)\right)
$$

to indicate the solution to Eq. (144). The instruction $\mathbb{T}$ indicates that the operators $\mathcal{S}_{\mathcal{Y}}\left(\bar{\mu}^{2} ; \nu\right)$ with the smallest values of $\bar{\mu}^{2}$ belong on the left.

We will sometimes adopt the notation

$$
\mathcal{I}(\nu)=\int_{\mu_{f}^{2}}^{Q^{2}} \frac{d \mu^{2}}{\mu^{2}} \mathcal{S}_{\mathcal{Y}}\left(\mu^{2} ; \nu\right)
$$

when the upper integration limit is $Q^{2}$ and we do not need to explicitly display $\mathcal{S}_{\mathcal{Y}}\left(\mu^{2} ; \nu\right){ }^{6}$

\section{B. Relation of $\mathcal{S}_{\mathcal{Y}}$ to the shower generator $\mathcal{S}$}

We can relate $\mathcal{S}_{\mathcal{Y}}\left(\mu^{2}, \nu\right)$ to $\mathcal{S}\left(\mu^{2}\right)$. From Eq. (105), we have

$$
\mu^{2} \frac{\partial}{\partial \mu^{2}} \mathcal{U}\left(\mu_{f}^{2}, \mu^{2}\right)=\mathcal{U}\left(\mu_{f}^{2}, \mu^{2}\right) \mathcal{S}\left(\mu^{2}\right) .
$$

Using Eqs. (147) and (144) to differentiate Eq. (139), we have

$$
\left(1 \mid \mathcal{Y}\left(\mu^{2} ; \nu\right) \mathcal{S}_{\mathcal{Y}}\left(\mu^{2} ; \nu\right)=\left(1 \mid \mathcal{O}(\nu) \mathcal{U}\left(\mu_{f}^{2}, \mu^{2}\right) \mathcal{S}\left(\mu^{2}\right) \mathcal{O}^{-1}(\nu)\right.\right.
$$

Using Eq. (140), this becomes

$$
\left(1 \mid \mathcal{Y}\left(\mu^{2} ; \nu\right) \mathcal{S}_{\mathcal{Y}}\left(\mu^{2} ; \nu\right)=\left(1 \mid \mathcal{Y}\left(\mu^{2} ; \nu\right) \mathcal{O}(\nu) \mathcal{S}\left(\mu^{2}\right) \mathcal{O}^{-1}(\nu)\right.\right.
$$

We can also use Eq. (144), together with the boundary condition (142), to write an equation for $\mathcal{Y}\left(\mu^{2} ; \nu\right)$,

$$
\mathcal{Y}\left(\mu^{2} ; \nu\right)=1+\int_{\mu_{f}^{2}}^{\mu^{2}} \frac{d \bar{\mu}^{2}}{\bar{\mu}^{2}} \mathcal{Y}\left(\bar{\mu}^{2} ; \nu\right) \mathcal{S}_{\mathcal{Y}}\left(\bar{\mu}^{2} ; \nu\right) \text {. }
$$

\footnotetext{
${ }^{6}$ This is a useful definition even though $\mathcal{Y}\left(\mu^{2} ; \nu\right)$ is not the exponential of $\mathcal{I}(\nu)$ because of the $\mathbb{T}$ instruction in Eq. (145).
} 


\section{Operator mapping $\mathbb{P}$}

To use Eq. (149), we introduce some useful notation, expanding on Sec. IV. Let $\mathcal{A}$ be an operator that increases the number of partons or leaves the number of partons unchanged and changes momenta, flavors, and colors. Let $\mathcal{B}$ be an operator on the statistical space that leaves the number $m$ of partons and their momenta and flavors $\{p, f\}_{m}$ unchanged, although it can change the parton color state. ${ }^{7}$ Let $\mathcal{B}$ be defined such that

$$
(1 \mid \mathcal{B}=(1 \mid \mathcal{A}
$$

We will define a linear relation $\mathcal{A} \rightarrow \mathcal{B}$ that realizes this relation. To represent this linear relation, we adopt the notation

$$
\mathcal{B}=[\mathcal{A}]_{\mathbb{P}}
$$

The needed construction is straightforward. Suppose that $\mathcal{A}$ maps states with $m$ partons into states with $\hat{m}$ partons, with $\hat{m} \geq m$. Let $\mathcal{A}$ have the form

$$
\mathcal{A}=\left(C_{\text {ket }} \otimes C_{\text {bra }}^{\dagger}\right) \mathcal{R},
$$

where $\mathcal{R}$ acts on the momentum and flavor factor of the statistical space and $\left(C_{\mathrm{ket}} \otimes C_{\mathrm{bra}}^{\dagger}\right)$ acts on the color factor. Recall from Sec. XII the meaning of the color operators $\left(C_{\text {ket }} \otimes C_{\text {bra }}^{\dagger}\right)$. Letting $\left|\{c\}_{m}\right\rangle$ and $\left|\left\{c^{\prime}\right\}_{m}\right\rangle$ be basis vectors for color amplitudes, a color basis vector $\left.\mid\left\{c, c^{\prime}\right\}_{m}\right)$ in the statistical space represents the color density operator $\left|\{c\}_{m}\right\rangle\left\langle\left\{c^{\prime}\right\}_{m}\right|$. Then $\left.\left(C_{\text {ket }} \otimes C_{\text {bra }}^{\dagger}\right) \mid\left\{c, c^{\prime}\right\}_{m}\right)$ represents the color density operator $C_{\text {ket }}\left|\{c\}_{m}\right\rangle\left\langle\left\{c^{\prime}\right\}_{m}\right| C_{\text {bra }}^{\dagger}$.

Let us evaluate $\left(1|\mathcal{A}|\left\{p, f, c, c^{\prime}\right\}_{m}\right)$ for an arbitrary $m$ parton basis state $\left.\mid\left\{p, f, c, c^{\prime}\right\}_{m}\right)$. The inner product of (1| with a statistical basis state is given in Eq. (103). We insert a sum over the basis states [19] with $\hat{m}$ partons,

$$
\begin{aligned}
(1|\mathcal{A}| & \left.\left\{p, f, c, c^{\prime}\right\}_{m}\right) \\
= & \frac{1}{\hat{m} !} \int\left[d\{p, f\}_{\hat{m}}\right] \sum_{\left\{c, c^{\prime}\right\}_{\hat{m}}}\left(1 \mid\left\{\hat{p}, \hat{f}, \hat{c}, \hat{c}^{\prime}\right\}_{\hat{m}}\right) \\
& \times\left(\left\{\hat{c}, \hat{c}^{\prime}\right\}_{\hat{m}}\left|C_{\mathrm{ket}} \otimes C_{\mathrm{bra}}^{\dagger}\right|\left\{c, c^{\prime}\right\}_{m}\right) \\
& \times\left(\{\hat{p}, \hat{f}\}_{\hat{m}}|\mathcal{R}|\{p, f\}_{m}\right) .
\end{aligned}
$$

For the color, this gives us the trace of the color density operator obtained by applying $C_{\mathrm{ket}} \otimes C_{\mathrm{bra}}^{\dagger}$ to $\left.\mid\left\{c, c^{\prime}\right\}_{m}\right)$, namely the trace of $C_{\text {ket }}\left|\{c\}_{m}\right\rangle\left\langle\left\{c^{\prime}\right\}_{m}\right| C_{\text {bra }}^{\dagger}$. The result is

\footnotetext{
${ }^{7}$ In Sec. IV, $\mathcal{A}$ is sometimes an operator that is defined in $d=4-2 \epsilon$ dimensions that contains poles $1 / \epsilon$ and singularities when the momenta of partons created by $\mathcal{A}$ become soft or collinear. However, $(1 \mid \mathcal{A}$ is well defined in $d=4$ dimensions. Then $\mathcal{B}$ is well defined in 4 dimensions.
}

$$
\begin{aligned}
(1|\mathcal{A}| & \left.\left\{p, f, c, c^{\prime}\right\}_{m}\right) \\
= & \left\langle\left\{c^{\prime}\right\}_{m}\left|C_{\mathrm{bra}}^{\dagger} C_{\mathrm{ket}}\right|\{c\}_{m}\right\rangle \\
& \times \frac{1}{\hat{m} !} \int\left[d\{p, f\}_{\hat{m}}\right]\left(\{\hat{p}, \hat{f}\}_{\hat{m}}|\mathcal{R}|\{p, f\}_{m}\right) .
\end{aligned}
$$

We now need to define $\mathcal{B}=[A]_{\mathbb{P}}$ so that

$$
\left(1 \mid[\mathcal{A}]_{\mathbb{P}}=(1 \mid \mathcal{A} \text {. }\right.
$$

We distinguish two cases. First, if $\hat{m}=m$ we leave the color operator in $\mathcal{A}$ unchanged,

$$
\begin{aligned}
{\left.\left[\left(C_{\mathrm{ket}} \otimes C_{\mathrm{bra}}^{\dagger}\right) \mathcal{R}\right]_{\mathbb{P}} \mid\left\{p, f, c, c^{\prime}\right\}_{m}\right) } \\
\left.=\left(C_{\mathrm{ket}} \otimes C_{\mathrm{bra}}^{\dagger}\right) \mid\left\{p, f, c, c^{\prime}\right\}_{m}\right) \\
\quad \times \frac{1}{m !} \int\left[d\{\hat{p}, \hat{f}\}_{m}\right]\left(\{\hat{p}, \hat{f}\}_{m}|\mathcal{R}|\{p, f\}_{m}\right) .
\end{aligned}
$$

Evidently, this satisfies $\left(1 \mid[\mathcal{A}]_{\mathbb{P}}=(1 \mid \mathcal{A}\right.$.

Second, if $\hat{m}>m$ we define, with one exception,

$$
\begin{aligned}
{\left.\left[\left(C_{\mathrm{ket}} \otimes C_{\mathrm{bra}}^{\dagger}\right) \mathcal{R}\right]_{\mathbb{P}} \mid\left\{p, f, c, c^{\prime}\right\}_{m}\right) } \\
\left.=\frac{1}{2}\left(C_{\mathrm{bra}}^{\dagger} C_{\mathrm{ket}} \otimes 1+1 \otimes C_{\mathrm{bra}}^{\dagger} C_{\mathrm{ket}}\right) \mid\left\{p, f, c, c^{\prime}\right\}_{m}\right) \\
\quad \times \frac{1}{\hat{m} !} \int\left[d\{\hat{p}, \hat{f}\}_{\hat{m}}\right]\left(\{\hat{p}, \hat{f}\}_{\hat{m}}|\mathcal{R}|\{p, f\}_{m}\right) .
\end{aligned}
$$

This also satisfies $\left(1 \mid[\mathcal{A}]_{\mathbb{P}}=(1 \mid \mathcal{A}\right.$.

The one exception concerns the first order splitting operator describing real emissions, $\mathcal{S}^{[1,0]}$. This operator contains a number of terms. There are some terms with color content that can be written in a shorthand notation as $t_{l}^{\dagger} \otimes t_{k}$. This describes the splitting of parton $l$ in the ket state interfering with the splitting of parton $k$ in the bra state. Parton $l$ is treated as the splitting parton in the momentum dependent part of the splitting function, while parton $k$ is the dipole partner parton. We can have $k=l$. When the newly created parton is a gluon, we can also have $k \neq l$. There are also terms in the splitting operator of the form $t_{k}^{\dagger} \otimes t_{l}$ in which the roles of the bra and ket color states are reversed. For $\mathcal{S}^{[1,0]}$, we define

$$
\begin{aligned}
& {\left[t_{l}^{\dagger} \otimes t_{k}\right]_{\mathbb{P}}=1 \otimes t_{k} t_{l}^{\dagger},} \\
& {\left[t_{k}^{\dagger} \otimes t_{l}\right]_{\mathbb{P}}=t_{l} t_{k}^{\dagger} \otimes 1 .}
\end{aligned}
$$

In the case $k=l$, the color operator is $\left(t_{l}^{\dagger} \otimes t_{k}+t_{k}^{\dagger} \otimes t_{l}\right) / 2$, so one simply averages over the two cases in Eq. (159). ${ }^{8}$ This asymmetric definition that depends on whether the dipole partner parton is in the bra state or the ket state

\footnotetext{
${ }^{8}$ Additionally, for $k=l$, the operators $t_{k} t_{l}^{\dagger}$ and $t_{l} t_{k}^{\dagger}$ are color Casimir operators, $C_{\mathrm{F}}, C_{\mathrm{A}}$, or $T_{\mathrm{R}}$, so the two cases in Eq. (159) are really the same.
} 
makes the definition of $\left[\mathcal{S}^{[1,0]}\right]_{\mathbb{P}}$ match the definition of the virtual splitting operator $\mathcal{S}^{[0,1]}$ in the LC+ approximation [22].

There is a special case of some importance. Suppose that $\hat{m}=m$ and, in addition, $\mathcal{A}$ leaves the momenta and flavors of all partons unchanged. That is, $\left.\mid\{p, f\}_{m}\right)$ is an eigenvector of $\mathcal{R}$ :

$$
\left.\left.\mathcal{R} \mid\{p, f\}_{m}\right)=r\left(\{p, f\}_{m}\right) \mid\{p, f\}_{m}\right) .
$$

Then $\mathcal{A}$ applied to $\left.\mid\left\{p, f, c, c^{\prime}\right\}_{m}\right)$ takes the form

$$
\begin{aligned}
\left.\mathcal{A} \mid\left\{p, f, c, c^{\prime}\right\}_{m}\right)= & \left(C_{\mathrm{ket}} \otimes C_{\mathrm{bra}}^{\dagger}\right) r\left(\{p, f\}_{m}\right) \\
& \left.\times \mid\left\{p, f, c, c^{\prime}\right\}_{m}\right) .
\end{aligned}
$$

In this case, the definition (157) gives us

$$
[\mathcal{A}]_{\mathbb{P}}=\mathcal{A} .
$$

There is some freedom available in fixing the color part of $\left[\left(C_{\mathrm{ket}} \otimes C_{\mathrm{bra}}^{\dagger}\right) \mathcal{R}\right]_{\mathbb{P}}$, as discussed in Sec. VID of Ref. [18]. We could add any operator $\mathcal{A}^{\prime}$ to $[\mathcal{A}]_{\mathbb{P}}$ if $\mathcal{A}^{\prime}$ has the property that $\left(1 \mid \mathcal{A}^{\prime}=0\right.$. The form in Eqs. (157), (158), and (159) is recommended by its simplicity, so we will use it in this paper.

This defines the operator $[\mathcal{A}]_{\mathbb{P}}$ in general. However, when $[\mathcal{A}]_{\mathbb{P}}$ acts on the $q \bar{q}$ initial hard scattering state in $e^{+} e^{-}$annihilation, the action of $[\mathcal{A}]_{\mathbb{P}}$ is simpler. The color space for $q \bar{q}$ contains only one basis vector, $\left.\mid\{c, c\}_{2}\right)$, with $\left\{c^{\prime}\right\}_{2}=\{c\}_{2}$ and $\left(1_{\text {color }} \mid\{c, c\}_{2}\right)=\left\langle\{c\}_{2} \mid\{c\}_{2}\right\rangle=1$. Therefore $C_{\mathrm{bra}}^{\dagger} C_{\mathrm{ket}} \otimes 1$ or $1 \otimes C_{\mathrm{bra}}^{\dagger} C_{\mathrm{ket}}$ acting on $\left.\mid\{c, c\}_{2}\right)$ can only return an eigenvalue:

$$
\begin{aligned}
{\left.\left[C_{\mathrm{bra}}^{\dagger} C_{\mathrm{ket}} \otimes 1\right] \mid\{c, c\}_{2}\right) } & \left.=\left[1 \otimes C_{\mathrm{bra}}^{\dagger} C_{\mathrm{ket}}\right] \mid\{c, c\}_{2}\right) \\
& \left.=\lambda_{\text {color }} \mid\{c, c\}_{2}\right),
\end{aligned}
$$

where

$$
\lambda_{\text {color }}=\left\langle\{c\}_{2}\left|C_{\mathrm{bra}}^{\dagger} C_{\mathrm{ket}}\right|\{c\}_{2}\right\rangle .
$$

This tells us that $\left.\mid\{p, f, c, c\}_{2}\right)$ is an eigenvector of $[\mathcal{A}]_{\mathbb{P}}$ :

$$
\left.\left.[\mathcal{A}]_{\mathbb{P}} \mid\{p, f, c, c\}_{2}\right)=\lambda_{\mathcal{A}} \mid\{p, f, c, c\}_{2}\right) .
$$

Using $\left(1 \mid\{p, f, c, c\}_{2}\right)=\left\langle\{c\}_{2} \mid\{c\}_{2}\right\rangle=1$, we have a very simple result for the eigenvalue,

$$
\lambda_{\mathcal{A}}=\left(1|\mathcal{A}|\{p, f, c, c\}_{2}\right)
$$

\section{Recursive definition of $\mathcal{S}_{\mathcal{Y}}$}

We can now define $\mathcal{S}_{\mathcal{Y}}\left(\mu^{2} ; \nu\right)$ so that it satisfies Eq. (149). Recall that $\mathcal{Y}\left(\mu^{2} ; \nu\right)=1+\mathcal{O}\left(\alpha_{s}\right)$. Because of this, it is possible to isolate $\mathcal{S}_{\mathcal{Y}}\left(\mu^{2} ; \nu\right)$ on the left-hand side of Eq. (149):

$$
\begin{aligned}
\left(1 \mid \mathcal{S}_{\mathcal{Y}}\left(\mu^{2} ; \nu\right)=\right. & \left(1 \mid\left\{\mathcal{Y}\left(\mu^{2} ; \nu\right) \mathcal{O}(\nu) \mathcal{S}\left(\mu^{2}\right) \mathcal{O}^{-1}(\nu)\right.\right. \\
& \left.+\left[1-\mathcal{Y}\left(\mu^{2} ; \nu\right)\right] \mathcal{S}_{\mathcal{Y}}\left(\mu^{2} ; \nu\right)\right\}
\end{aligned}
$$

Using the operator mapping $[\cdots]_{\mathbb{P}}$, this is

$$
\begin{aligned}
\mathcal{S}_{\mathcal{Y}}\left(\mu^{2} ; \nu\right)= & {\left[\mathcal{Y}\left(\mu^{2} ; \nu\right) \mathcal{O}(\nu) \mathcal{S}\left(\mu^{2}\right) \mathcal{O}^{-1}(\nu)\right]_{\mathbb{P}} } \\
& +\left[\left[1-\mathcal{Y}\left(\mu^{2} ; \nu\right)\right] \mathcal{S}_{\mathcal{Y}}\left(\mu^{2} ; \nu\right)\right]_{\mathbb{P}}
\end{aligned}
$$

Note that the operators $\mathcal{Y}\left(\mu^{2} ; \nu\right)$ and $\mathcal{S}_{\mathcal{Y}}\left(\mu^{2} ; \nu\right)$ in the second line of Eq. (168) leave the number of partons, their momenta, and their flavors unchanged. Thus Eq. (162) applies and the $[\cdots]_{\mathbb{P}}$ operation has no effect.

Equation (168) can be used to define $\mathcal{S}_{\mathcal{Y}}\left(\mu^{2} ; \nu\right)$ and $\mathcal{Y}\left(\mu^{2} ; \nu\right)$ recursively. We can write $\mathcal{S}_{\mathcal{Y}}\left(\mu^{2} ; \nu\right), \mathcal{Y}\left(\mu^{2} ; \nu\right)$, and $\mathcal{I}(\nu)$ as expansions in powers of the shower evolution operator $\mathcal{S}$ :

$$
\begin{aligned}
\mathcal{S}_{\mathcal{Y}}\left(\mu^{2} ; \nu\right) & =\sum_{k=1}^{\infty} \mathcal{S}_{\mathcal{Y}}^{[k]}\left(\mu^{2} ; \nu\right), \\
\mathcal{Y}\left(\mu^{2} ; \nu\right) & =1+\sum_{k=1}^{\infty} \mathcal{Y}^{[k]}\left(\mu^{2} ; \nu\right), \\
\mathcal{I}(\nu) & =\sum_{k=1}^{\infty} \mathcal{I}^{[k]}(\nu),
\end{aligned}
$$

where each of $\mathcal{S}_{\mathcal{Y}}^{[k]}\left(\mu^{2} ; \nu\right), \mathcal{Y}^{[k]}\left(\mu^{2} ; \nu\right)$, and $\mathcal{I}^{[k]}(\nu)$ contain $k$ factors of $\mathcal{S}$. Then we can write Eq. (168) as

$$
\begin{aligned}
\mathcal{S}_{\mathcal{Y}}^{[k]}\left(\mu^{2} ; \nu\right)= & {\left[\mathcal{Y}^{[k-1]}\left(\mu^{2} ; \nu\right) \mathcal{O}(\nu) \mathcal{S}\left(\mu^{2}\right) \mathcal{O}^{-1}(\nu)\right]_{\mathbb{P}} } \\
& -\sum_{j=1}^{k-1} \mathcal{Y}^{[k-j]}\left(\mu^{2} ; \nu\right) \mathcal{S}_{\mathcal{Y}}^{[j]}\left(\mu^{2} ; \nu\right)
\end{aligned}
$$

Similarly, we can write Eq. (150) as

$$
\mathcal{Y}^{[k]}\left(\mu^{2} ; \nu\right)=\sum_{j=1}^{k} \int_{\mu_{\mathrm{f}}^{2}}^{\mu^{2}} \frac{d \bar{\mu}^{2}}{\bar{\mu}^{2}} \mathcal{Y}^{[k-j]}\left(\bar{\mu}^{2} ; \nu\right) \mathcal{S}_{\mathcal{Y}}^{[j]}\left(\bar{\mu}^{2} ; \nu\right)
$$

These equations apply for $k=1,2, \ldots$ with $\mathcal{Y}^{[0]}\left(\mu^{2} ; \nu\right)=1$.

We now illustrate this for the first two orders. At order 1 , Eq. (170) gives us

$$
\mathcal{S}_{\mathcal{Y}}^{[1]}\left(\mu^{2} ; \nu\right)=\left[\mathcal{O}(\nu) \mathcal{S}\left(\mu^{2}\right) \mathcal{O}^{-1}(\nu)\right]_{\mathbb{P}} .
$$

At order $\alpha_{s}^{2}$, we have 


$$
\begin{aligned}
\mathcal{S}_{\mathcal{Y}}^{[2]}\left(\mu^{2} ; \nu\right)= & {\left[\mathcal{Y}^{[1]}\left(\mu^{2} ; \nu\right) \mathcal{O}(\nu) \mathcal{S}\left(\mu^{2}\right) \mathcal{O}^{-1}(\nu)\right]_{\mathbb{P}} } \\
& -\mathcal{Y}^{[1]}\left(\mu^{2} ; \nu\right) \mathcal{S}_{\mathcal{Y}}^{[1]}\left(\mu^{2} ; \nu\right) .
\end{aligned}
$$

From Eq. (150) at first order, we have

$$
\mathcal{Y}^{[1]}\left(\mu^{2} ; \nu\right)=\int_{\mu_{\mathrm{f}}^{2}}^{\mu^{2}} \frac{d \bar{\mu}^{2}}{\bar{\mu}^{2}} \mathcal{S}_{\mathcal{Y}}^{[1]}\left(\bar{\mu}^{2} ; \nu\right) .
$$

For $\mathcal{S}_{\mathcal{Y}}^{[1]}\left(\mu^{2} ; \nu\right)$ we can use Eq. (172). This gives us

$$
\begin{aligned}
\mathcal{S}_{\mathcal{Y}}^{[2]}\left(\mu^{2} ; \nu\right)= & \int_{\mu_{\mathrm{f}}^{2}}^{\mu^{2}} \frac{d \bar{\mu}^{2}}{\bar{\mu}^{2}}\left[\left[\mathcal{O}(\nu) \mathcal{S}\left(\bar{\mu}^{2}\right) \mathcal{O}^{-1}(\nu)\right]_{\mathbb{P}}\right. \\
& \left.\times\left[\mathcal{O}(\nu) \mathcal{S}\left(\mu^{2}\right) \mathcal{O}^{-1}(\nu)\right]_{1-\mathbb{P}}\right]_{\mathbb{P}} .
\end{aligned}
$$

Here we use the abbreviation

$$
[\mathcal{A}]_{1-\mathbb{P}}=\mathcal{A}-[\mathcal{A}]_{\mathbb{P}} .
$$

The operator $\mathcal{S}_{\mathcal{Y}}\left(\mu^{2} ; \nu\right)$ is a complicated operator in general. However, it is significant that, because of Eqs. (165) and (166), the initial $q \bar{q}$ state is an eigenvector of $\mathcal{S}_{\mathcal{Y}}\left(\mu^{2} ; \nu\right)$ :

$$
\left.\left.\mathcal{S}_{\mathcal{Y}}\left(\mu^{2} ; \nu\right) \mid\{p, f, c, c\}_{2}\right)=\lambda_{\mathcal{S}_{\mathcal{Y}}} \mid\{p, f, c, c\}_{2}\right),
$$

where

$$
\lambda_{\mathcal{S}_{\mathcal{Y}}}=\left(1\left|\mathcal{S}_{\mathcal{Y}}\left(\mu^{2} ; \nu\right)\right|\{p, f, c, c\}_{2}\right) .
$$

\section{EVALUATION OF $\mathcal{S}_{\mathcal{Y}}^{[1]}\left(\mu^{2} ; \nu\right)$}

Let us see what we can say about $\mathcal{S}_{\mathcal{Y}}^{[1]}\left(\mu^{2} ; \nu\right)$ as given in Eq. (172). In a first order shower, like Deductor, we divide $S\left(\mu^{2}\right)$ into its real emission and virtual parts as in Eq. (107). Then Eq. (172) gives us

$$
\begin{aligned}
\mathcal{S}_{\mathcal{Y}}^{[1]}\left(\mu^{2} ; \nu\right)= & {\left[\mathcal{O}(\nu) \mathcal{S}^{[1,0]}\left(\mu^{2}\right) \mathcal{O}^{-1}(\nu)\right.} \\
& \left.+\mathcal{O}(\nu) \mathcal{S}^{[0,1]}\left(\mu^{2}\right) \mathcal{O}^{-1}(\nu)\right]_{\mathbb{P}}
\end{aligned}
$$

The virtual operator $\mathcal{S}^{[0,1]}\left(\mu^{2} ; 0\right)$ leaves the momentum and flavor state unchanged, so this is

$\mathcal{S}_{\mathcal{Y}}^{[1]}\left(\mu^{2} ; \nu\right)=\left[\mathcal{O}(\nu) \mathcal{S}^{[1,0]}\left(\mu^{2}\right) \mathcal{O}^{-1}(\nu)+\mathcal{S}^{[0,1]}\left(\mu^{2}\right)\right]_{\mathbb{P}}$.

Recall from Eq. (108) that $\left(1 \mid \mathcal{S}^{[0,1]}\left(\mu^{2}\right)=-\left(1 \mid \mathcal{S}^{[1,0]}\left(\mu^{2}\right)\right.\right.$. This tells us that

$$
\left[\mathcal{S}^{[0,1]}\left(\mu^{2}\right)\right]_{\mathbb{P}}=-\left[\mathcal{S}^{[1,0]}\left(\mu^{2}\right)\right]_{\mathbb{P}} .
$$

Using Eq. (181), Eq. (180) becomes
$\mathcal{S}_{\mathcal{Y}}^{[1]}\left(\mu^{2} ; \nu\right)=\left[\mathcal{O}(\nu) \mathcal{S}^{[1,0]}\left(\mu^{2}\right) \mathcal{O}^{-1}(\nu)-\mathcal{S}^{[1,0]}\left(\mu^{2}\right)\right]_{\mathbb{P}}$.

This is a convenient form for calculations.

\section{CHANGE IN $\tau$ INDUCED BY A SPLITTING}

The operator $\mathcal{O}(\nu) \mathcal{S}^{[1,0]}\left(\mu^{2}\right) \mathcal{O}^{-1}(\nu)$ appears in Eq. (182) for $\mathcal{S}_{\mathcal{Y}}^{[1]}\left(\mu^{2} ; \nu\right)$. This operator is

$$
\mathcal{O}(\nu) \mathcal{S}^{[1,0]}\left(\mu^{2}\right) \mathcal{O}^{-1}(\nu)=e^{-\nu \tau_{\mathrm{op}}} \mathcal{S}^{[1,0]}\left(\mu^{2}\right) e^{+\nu \tau_{\mathrm{op}}}
$$

The operator $\mathcal{S}^{[1,0]}\left(\mu^{2}\right)$ is a sum of operators,

$$
\mathcal{S}^{[1,0]}\left(\mu^{2}\right)=\sum_{l=1}^{\infty} \mathcal{S}_{l}^{[1,0]}\left(\mu^{2}\right),
$$

where $l$ is the label of the parton that splits. When we apply $\mathcal{S}_{l}^{[1,0]}\left(\mu^{2}\right)$ to a state $\left.\mid\left\{p, f, c, c^{\prime}\right\}_{m}\right)$, the splitting operator creates a new state $\left.\mid\left\{\hat{p}, \hat{f}, \hat{c}, \hat{c}^{\prime}\right\}_{m+1}\right)$ as long as $l \leq m$. For $l>m, \mathcal{S}_{l}$ just gives zero. The operators $\tau_{\text {op }}$ measure the values of $\tau$ before and after the splitting. Thus

$$
\begin{aligned}
& \left(\left\{\hat{p}, \hat{f}, \hat{c}, \hat{c}^{\prime}\right\}_{m+1}\left|\mathcal{O}(\nu) \mathcal{S}_{l}^{[1,0]}\left(\mu^{2}\right) \mathcal{O}^{-1}(\nu)\right|\left\{p, f, c, c^{\prime}\right\}_{m}\right) \\
& \quad=e^{-\nu(\hat{\tau}-\tau)}\left(\left\{\hat{p}, \hat{f}, \hat{c}, \hat{c}^{\prime}\right\}_{m+1}\left|\mathcal{S}_{l}^{[1,0]}\left(\mu^{2}\right)\right|\left\{p, f, c, c^{\prime}\right\}_{m}\right),
\end{aligned}
$$

where $\tau=\tau\left(\{p\}_{m}\right)$ and $\hat{\tau}=\tau\left(\{\hat{p}\}_{m+1}\right)$. Thus we need to know how $\tau$ changes in a splitting. We are looking for the leading contributions to logarithms of $\nu$, so we can use the approximations that $\tau$ is small and that the splitting is nearly soft or collinear.

We start with momenta $\{p\}_{m}$ and suppose that the parton that splits is in the right thrust hemisphere, $l \in R$. The splitting produces a new parton $l$ and a parton $m+1$. After the splitting, we have partons with momenta $\{\hat{p}\}_{m+1}$.

The emission of a parton changes the thrust axis. However, in the case of a nearly soft or collinear splitting of a parton in a state with small $\tau$, the sum of the momenta of the daughter partons is very close to the momentum of the mother parton, so that the thrust axis changes by very little [35]. For this reason, we calculate $\tau\left(\{\hat{p}\}_{m+1}\right)$ for the new parton state using the thrust axis of the old parton state $\{p\}_{m}$. We also assume that after the splitting partons $l$ and $m+1$ are still in the right thrust hemisphere.

Now we turn to the calculation of $\hat{\tau}-\tau$. We use the definition, Eqs. (123) and (124), to write 
$\hat{\tau}-\tau=\frac{\hat{p}_{l}^{-}+\hat{p}_{m+1}^{-}-p_{l}^{-}}{Q^{-}}+\sum_{\substack{i \in R \\ i \neq\{l, m+1\}}} \frac{\hat{p}_{i}^{-}-p_{i}^{-}}{Q^{-}}+\sum_{i \in L} \frac{\hat{p}_{i}^{+}-p_{i}^{+}}{Q^{+}}$.

Now we need to evaluate $\left(\hat{p}_{l}^{-}+\hat{p}_{m+1}^{-}-p_{i}^{-}\right) / Q^{-}$and $\left(\hat{p}_{i}^{ \pm}-p_{i}^{ \pm}\right) / Q^{ \pm}$. Following the notation of Appendix B of Ref. [25], we define

$$
\begin{aligned}
h_{ \pm} & =(1+y \pm \lambda) / 2, \\
\lambda & =\sqrt{(1+y)^{2}-4 a_{l} y}, \\
a_{l} & =\frac{Q^{2}}{2 p_{l} \cdot Q},
\end{aligned}
$$

where $y$ was defined in Eq. (99). We suppose that $y \ll 1$. We define a lightlike vector $n_{l}$ by

$$
n_{l}=\frac{2 p_{l} \cdot Q}{Q^{2}} Q-p_{l} \text {. }
$$

Note that $n_{l}$ is independent of the normalization of $Q$.

We write the momentum vectors for partons $l$ and $m+1$ after the splitting as

$$
\begin{aligned}
\hat{p}_{l} & =h_{+} z p_{l}+h_{-}(1-z) n_{l}+k_{\perp}, \\
\hat{p}_{m+1} & =h_{+}(1-z) p_{l}+h_{-} z n_{l}-k_{\perp},
\end{aligned}
$$

where $k_{\perp} \cdot p_{l}=k_{\perp} \cdot n_{l}=0$. The splitting is specified by $y$, the momentum fraction $z$ in Eq. (189), and the azimuthal angle $\phi$ of $k_{\perp}$. The magnitude of $k_{\perp}$ is determined by the condition $\hat{p}_{l}^{2}=0$ or $\hat{p}_{m+1}^{2}=0$ :

$$
-k_{\perp}^{2}=z(1-z) y 2 p_{l} \cdot Q
$$

Define

$$
P_{l}=\hat{p}_{l}+\hat{p}_{m+1}=h_{+} p_{l}+h_{-} n_{l} .
$$

This gives us $P_{l}^{2}=2 p_{l} \cdot Q y$. Using these results we obtain

$$
\left(Q-p_{l}\right)^{2}=\left(Q-P_{l}\right)^{2} .
$$

We require that momentum be conserved in the splitting, so that

$$
Q-p_{l}=\sum_{\substack{i=1 \\ i \neq l}}^{m} p_{i}, \quad Q-P_{l}=\sum_{\substack{i=1 \\ i \neq l}}^{m} \hat{p}_{i}
$$

The relation (192) allows the $\hat{p}_{i}$ for $i \notin\{l, m+1\}$ to be obtained from the $p_{i}$ by a Lorentz transformation,

$$
\hat{p}_{i}^{\mu}=\Lambda_{\nu}^{\mu} p_{i}^{\nu}, \quad i \notin\{l, m+1\} .
$$

The needed Lorentz transformation can be a small boost in the $p_{l}-Q$ plane. Let

$$
p_{i}=\alpha_{i} p_{l}+\beta_{i} n_{l}+p_{i, \perp}
$$

where $p_{i, \perp} \cdot p_{l}=p_{i, \perp} \cdot n_{l}=0$. Then define $\hat{p}_{i}$ for $i \notin$ $\{l, m+1\}$ by

$$
\hat{p}_{i}=e^{\omega} \alpha_{i} p_{l}+e^{-\omega} \beta_{i} n_{l}+p_{i, \perp} .
$$

The needed boost angle is small:

$$
\omega=y+\mathcal{O}\left(y^{2}\right)
$$

Using Eq. (194) in Eq. (186), we have

$$
\begin{aligned}
\hat{\tau}-\tau= & \frac{\hat{p}_{l}^{-}+\hat{p}_{m+1}^{-}-p_{l}^{-}}{Q^{-}}+\sum_{\substack{i \in R \\
i \neq l}}\left(\Lambda_{\nu}^{-}-\delta_{\nu}^{-}\right) \frac{p_{i}^{\nu}}{Q^{-}} \\
& +\sum_{i \in L}\left(\Lambda_{\nu}^{+}-\delta_{\nu}^{+}\right) \frac{p_{i}^{\nu}}{Q^{+}} .
\end{aligned}
$$

We will see momentarily that $\left(\hat{p}_{l}^{-}+\hat{p}_{m+1}^{-}-p_{l}^{-}\right) / Q^{-}$is small, of order $y$. This allows $\hat{\tau}-\tau$ to be of order $y$.

In the third term, for $i \in \mathrm{R},\left(\Lambda_{\nu}^{\mu}-\delta_{\nu}^{\mu}\right)$ is of order $y$. The thrust axis defines the \pm components of vectors in Eq. (198). If $p_{l}$ were exactly aligned with the thrust axis, then the only nonvanishing index choice for $\Lambda_{\nu}^{-}$would be $\nu=-$. But $p_{i}^{-} / Q^{-} \ll 1$ for $i \in \mathrm{R}$, since this quantity is of order $\tau$ and we suppose that $\tau \ll 1$. This restriction on the index choices is not exact. However, for $i \in \mathrm{R}$, the components $p_{i}^{\nu} / Q^{-}$for $\nu \in\{1,2\}$ are of order $p_{i}^{\nu} / Q^{-} \sim\left[p_{i}^{+} p_{i}^{-}\right]^{1 / 2} / Q^{-}$, which is at most of order $\sqrt{\tau}$. The component $p_{i}^{\nu} / Q^{-}$for $\nu=+$ can be of order 1 . However, $\Lambda_{+}^{-}=\Lambda^{--}$is at most of order $y^{2}$ since $\Lambda=$ $\exp (\omega w)$ where $\omega$ is given by Eq. (197) and the first order contribution to $\Lambda^{--}$vanishes because the generator matrix $w^{\mu \nu}$ is antisymmetric. Thus the second term in Eq. (198) is of order $y$ times a small factor, either $\tau, \sqrt{\tau}$, or $y$. The same reasoning applies to the third term.

We conclude that the only surviving term in Eq. (198) is the first:

$$
\hat{\tau}-\tau \approx \frac{\hat{p}_{l}^{-}+\hat{p}_{m+1}^{-}-p_{l}^{-}}{Q^{-}} .
$$

We have

$$
\frac{\hat{p}_{l}^{-}+\hat{p}_{m+1}^{-}-p_{l}^{-}}{Q^{-}} \approx \frac{\left(1-a_{l}\right) y p_{l}^{-}+a_{l} y n_{l}^{-}}{Q^{-}} .
$$

With our kinematic conventions, 


$$
\begin{aligned}
& \frac{p_{l}^{-}}{Q^{-}}=\frac{1-\cos \theta\left(l, \vec{n}_{\mathrm{T}}\right)}{2 a_{l}}, \\
& \frac{n_{l}^{-}}{Q^{-}}=\frac{1+\cos \theta\left(l, \vec{n}_{\mathrm{T}}\right)}{2 a_{l}},
\end{aligned}
$$

where

$$
\cos \theta\left(l, \vec{n}_{\mathrm{T}}\right)=\frac{\left|\vec{p}_{l} \cdot \vec{n}_{\mathrm{T}}\right|}{\left|\vec{p}_{l}\right|}
$$

This gives us

$$
\frac{\hat{p}_{l}+\hat{p}_{m+1}^{-}-p_{l}^{-}}{Q^{-}} \approx \xi_{l} y
$$

where

$$
\xi_{l}=1-\left(1-\frac{1}{2 a_{l}}\right)\left[1-\cos \left(\theta\left(l, \vec{n}_{\mathrm{T}}\right)\right)\right]
$$

That is

$$
\hat{\tau}-\tau \approx \xi_{l} y .
$$

The same result holds for $l \in L$ if we change $1-\cos \left(\theta\left(l, \vec{n}_{\mathrm{T}}\right)\right)$ to $1+\cos \left(\theta\left(l, \vec{n}_{\mathrm{T}}\right)\right)$.

If we are splitting the quark or the antiquark in the two parton state created initially in $e^{+} e^{-}$annihilation, then $a_{l}=1$ and $\theta\left(l, \vec{n}_{\mathrm{T}}\right)=0$. Then $\xi_{l}=1$.

In the general case, $0<1-\cos \left(\theta\left(l, \vec{n}_{\mathrm{T}}\right)\right)<1$ for $l \in R$ and $1 / 2<\left(2 a_{l}-1\right) /\left(2 a_{l}\right)<1$, so

$$
0<\xi_{l}<1
$$

We get $\xi_{l} \rightarrow 0$ only when $\theta_{l} \rightarrow \pi / 2$ and parton $l$ is very soft, $1 / a_{l} \rightarrow 0$. Notice that there is no singularity for $\theta_{l} \rightarrow \pi / 2$, so there is no singularity for $\xi_{l} \rightarrow 0$. There is a singularity for $\theta\left(l, \vec{n}_{\mathrm{T}}\right) \rightarrow 0$ for all partons $l$. This corresponds to $\xi_{l} \rightarrow 1$. Thus in the general case we can treat $\xi_{l}$ as being close to 1 . We will argue in Appendix A that for the purpose of finding next-to-leading logarithms of $\nu$ we can simply set $\xi_{l}$ to 1 .

We conclude that the effect of the operators $\mathcal{O}(\nu)$ in a splitting of parton $l$ can be approximated by

$$
\begin{array}{r}
\left.\mathcal{O}(\nu) \mathcal{S}_{l}^{[1,0]}\left(\mu^{2}\right) \mathcal{O}^{-1}(\nu) \mid\left\{p, f, c, c^{\prime}\right\}_{m}\right) \\
\left.\quad \approx \mathcal{S}_{l}^{[1,0]}\left(\mu^{2}\right) e^{-\nu \xi_{l}^{\mathrm{op} y}} \mid\left\{p, f, c, c^{\prime}\right\}_{m}\right),
\end{array}
$$

where $\xi_{l}^{\mathrm{op}}$ is an operator that, acting on a state $\left.\mid\left\{p, f, c, c^{\prime}\right\}_{m}\right)$, has eigenvalue $\xi_{l}$ as defined in Eq. (204) as long as $l \leq m$. For $l>m$, we can simply define $\xi_{l}$ to have eigenvalue 1 . We recall that $\xi_{l}$ is generally of order 1 and equals 1 exactly in the case of a splitting of one of the partons in a two parton state. Using this in Eq. (182) gives us

$$
\mathcal{S}_{\mathcal{Y}}^{[1]}\left(\mu^{2} ; \nu\right) \approx-\sum_{l}\left[\mathcal{S}_{l}^{[1,0]}\left(\mu^{2}\right)\right]_{\mathbb{P}}\left(1-e^{-\nu \xi_{l}^{\mathrm{op} y}}\right) .
$$

\section{XIX. $\mathcal{S}_{\mathcal{Y}}^{[1]}$ FOR A QUARK-ANTIQUARK STATE}

For the $q \bar{q}$ state created initially in electron-positron annihilation, Eq. (208) simplifies considerably. First, the index $l$ denoting the parton that splits can take only the values $l=1$ (for the quark) and $l=2$ (for the antiquark). Each choice gives the same result, so we can take $l=1$ and multiply by two. Also, the color factors are trivial. In $\left[\mathcal{S}_{l}^{[1,0]}\left(\mu^{2}\right)\right]_{\mathbb{P}}$ we encounter color operators $\boldsymbol{T}_{1} \cdot \boldsymbol{T}_{1}, \boldsymbol{T}_{2} \cdot \boldsymbol{T}_{2}$, and $\boldsymbol{T}_{1} \cdot \boldsymbol{T}_{2}$, where $\boldsymbol{T}_{i} \cdot \boldsymbol{T}_{j}=\sum_{a} T_{i}^{a} T_{j}^{a}$ and $T_{i}^{a}$ inserts a color matrix $T^{a}$ on parton line $i$. The operators $\boldsymbol{T}_{1} \cdot \boldsymbol{T}_{1}$ and $\boldsymbol{T}_{2} \cdot \boldsymbol{T}_{2}$ simply give an eigenvalue $C_{\mathrm{F}}$ times the unit color operator, while $\boldsymbol{T}_{1} \cdot \boldsymbol{T}_{2}$ gives $-C_{\mathrm{F}}$. This gives us a result of the form

$$
\begin{aligned}
& \left.\mathcal{S}_{\mathcal{Y}}^{[1]}\left(\mu^{2} ; \nu\right) \mid\left\{p, f, c, c^{\prime}\right\}_{2}\right) \\
& \left.\quad \approx-\left(1-e^{-\nu y}\right) \lambda(y) \mid\left\{p, f, c, c^{\prime}\right\}_{2}\right) .
\end{aligned}
$$

The eigenvalue $\lambda(y)$ is obtained in a straightforward calculation from the $q \rightarrow q+g$ splitting functions used in DeDuctor [22]. There is an integral over the splitting variables $z$ and $\phi$. The $\phi$ integral is trivial and gives simply a factor $2 \pi$. The integration over the momentum fraction $z$ remains,

$$
\begin{aligned}
\lambda(y)= & 2 C_{\mathrm{F}} \int_{0}^{1} d z\left\{\frac{\alpha_{s}\left(\lambda_{\mathrm{R}}(1-z) y Q^{2}\right)}{2 \pi} f_{\text {sing }}(z, y)\right. \\
& \left.+\frac{\alpha_{s}\left(\lambda_{\mathrm{R}} y Q^{2}\right)}{2 \pi} f_{\text {reg }}(z, y)\right\} .
\end{aligned}
$$

The argument of $\alpha_{s}$ contains the standard factor $\lambda_{R}$, Eq. (110), and, in the first term, a factor $(1-z)$, as in Eq. (109) with $a_{l}=1$. The functions $f_{\text {sing }}(z, y)$ and $f_{\text {reg }}(z, y)$ are taken directly from DEDUCTOR and are quite complicated. However, they are simple in the relevant limits, $y \rightarrow 0$ with fixed $z$ and $y \rightarrow 0$ with $1-z \propto y$. In these limits, they are

$$
\begin{aligned}
f_{\text {sing }}(z, y) & \approx \frac{2}{1-z+y}-2, \\
f_{\text {reg }}(z, y) & \approx 1-z .
\end{aligned}
$$

Note that $f_{\text {sing }}(z, 0)+f_{\text {reg }}(z)=\left(1+z^{2}\right) /(1-z)$ is just the DGLAP splitting kernel for $q \rightarrow q+\mathrm{g}$. However in $f_{\text {sing }}(z, y)$ the singularity at $(1-z) \rightarrow 0$ is regulated by adding $y$ in the denominator. 
We have written these results in the form used in DEDUCTOR. In $f_{\text {sing }}(z, y)$, we could recognize that the second term could have been transferred to $f_{\text {reg }}(z, y)$.

We would now like to compare this to the standard results for the summation of $\operatorname{logs}$ of $\tau$ in Ref. [35]. We begin by inserting Eq. (211) into Eq. (210):

$$
\begin{aligned}
\lambda(y) \approx & 2 C_{\mathrm{F}} \int_{0}^{1} d z\left\{\frac{\alpha_{s}\left(\lambda_{\mathrm{R}}(1-z) y Q^{2}\right)}{2 \pi} \frac{2}{1-z+y}\right. \\
& \left.-\frac{\alpha_{s}\left(\lambda_{\mathrm{R}}(1-z) y Q^{2}\right)}{2 \pi} 2+\frac{\alpha_{s}\left(\lambda_{\mathrm{R}} y Q^{2}\right)}{2 \pi}(1-z)\right\} .
\end{aligned}
$$

We will want to evaluate this approximately for small $y$ in such a way that if we expand the result in powers of $\alpha_{s}\left(y Q^{2}\right)$ we retain all terms proportional to $\alpha_{s}^{n}\left(y Q^{2}\right) \log ^{n}(y)$ and $\alpha_{s}^{n}\left(y Q^{2}\right) \log ^{n-1}(y)$. After integrating over $\bar{\mu}^{2}=y Q^{2}$ as in Eq. (145), this will give contributions $\alpha_{s}^{n}\left(Q^{2}\right) \log ^{n+1}(\nu)$ and $\alpha_{s}^{n}\left(Q^{2}\right) \log ^{n}(\nu)$. These are the leading $\log (\mathrm{LL})$ and next-to-leading $\log$ (NLL) terms. In $\lambda(y)$, we neglect contributions proportional to fewer powers of $\log (y)$ or to powers of $y$.

In order to carry out this approximate evaluation, we note first that we can use

$$
\alpha_{s}\left(A \mu^{2}\right)=\alpha_{s}\left(\mu^{2}\right)-\beta_{0} \log (A) \alpha_{s}^{2}\left(\mu^{2}\right)+\mathcal{O}\left(\alpha_{s}^{3}\right),
$$

where $\beta_{0}=\left(11 C_{\mathrm{A}}-2 n_{f}\right) /(12 \pi)$. Then we can omit the $\lambda_{\mathrm{R}}(1-z)$ factor in the argument of $\alpha_{s}$ in the second term in Eq. (212) and the $\lambda_{\mathrm{R}}$ in the third term, since these terms do not have $1 /(1-z+y)$ singularities that could produce $\log (y)$ factors after integration. In the first term, there is a $1 /(1-z+y)$ singularity. For this term, we need to keep the $\alpha_{s}^{2}$ contribution in Eq. (213). After performing the $z$ integration in the last two terms, this gives us

$$
\begin{aligned}
\lambda(y) \approx & 4 C_{\mathrm{F}} \int_{0}^{1} d(1-z) \frac{1}{1-z+y} \\
& \times \frac{\alpha_{s}\left((1-z) y Q^{2}\right)-\beta_{0} \log \left(\lambda_{\mathrm{R}}\right) \alpha_{s}^{2}\left((1-z) y Q^{2}\right)}{2 \pi} \\
& -3 C_{\mathrm{F}} \frac{\alpha_{s}\left(y Q^{2}\right)}{2 \pi} .
\end{aligned}
$$

Now we note that the $y$ in the denominator in the first term of Eq. (214) places an effective lower cutoff on $(1-z)$ at about $(1-z)=y$. This observation suggests that the integration over $(1-z)$ can be written in a simpler form:

$$
\begin{aligned}
\lambda(y) \approx & 4 C_{\mathrm{F}} \int_{y}^{1} \frac{d(1-z)}{1-z} \\
& \times \frac{\alpha_{s}\left((1-z) y Q^{2}\right)-\beta_{0} \log \left(\lambda_{\mathrm{R}}\right) \alpha_{s}^{2}\left((1-z) y Q^{2}\right)}{2 \pi} \\
& -3 C_{\mathrm{F}} \frac{\alpha_{s}\left(y Q^{2}\right)}{2 \pi}
\end{aligned}
$$

In fact, this correctly reproduces the $\alpha_{s}^{n} \log ^{n}(y)$ terms and the $\alpha_{s}^{n} \log ^{n-1}(y)$ terms in the expansion of the integral. To see this, one can approximately solve the renormalization group equation for $\alpha_{s}$ in the form [36]

$$
\begin{aligned}
\frac{1}{\alpha_{s}\left(A \mu^{2}\right)}= & \frac{1+\beta_{0} \log (A) \alpha_{s}\left(\mu^{2}\right)}{\alpha_{s}\left(\mu^{2}\right)} \\
& +\frac{\beta_{1}}{\beta_{0}} \log \left(1+\beta_{0} \log (A) \alpha_{s}\left(\mu^{2}\right)\right)+\cdots,
\end{aligned}
$$

with $\mu^{2}=y Q^{2}$ and $A=1-z$. Here $\beta_{1}=\left(17 C_{\mathrm{A}}^{2}-5 C_{\mathrm{A}} n_{f}-\right.$ $\left.3 C_{\mathrm{F}} n_{f}\right) /\left(24 \pi^{2}\right)$. This yields $\alpha_{s}\left(A \mu^{2}\right)$ as a series

$$
\begin{aligned}
\alpha_{s}\left(A \mu^{2}\right)= & \alpha_{s}\left(\mu^{2}\right)\left\{1+\sum_{n=2}^{\infty} \alpha_{s}^{n}\left(\mu^{2}\right)\left[c_{n} \log ^{n}(A)\right.\right. \\
& \left.\left.+d_{n} \log ^{n-1}(A)+\cdots\right]\right\} .
\end{aligned}
$$

Then one can check that the integral (214) agrees with the integral (215) at the NLL level.

The current code in DEDUCTOR does not include the $\beta_{1}$ contributions in evaluating the $z$ dependence of $\alpha_{s}\left((1-z) y Q^{2}\right)$. This appears to be not particularly significant numerically, but it is significant in principle because it means that some of the NLL contributions to $\mathcal{S}_{\mathcal{Y}}^{[1]}\left(\mu^{2} ; \nu\right)$ are absent.

We can now compare to Ref. [35] by changing the integration variable to $q^{2}=(1-z) y Q^{2}$ :

$$
\begin{aligned}
\lambda(y) \approx & 4 C_{\mathrm{F}} \int_{y^{2} Q^{2}}^{y Q^{2}} \frac{d q^{2}}{q^{2}} \frac{\alpha_{s}\left(q^{2}\right)-\beta_{0} \log \left(\lambda_{\mathrm{R}}\right) \alpha_{s}^{2}\left(q^{2}\right)}{2 \pi} \\
& -3 C_{\mathrm{F}} \frac{\alpha_{s}\left(y Q^{2}\right)}{2 \pi} .
\end{aligned}
$$

This agrees with the result in Eq. (64) of Ref. [35] for the LL and NLL contributions to $\lambda(y)$.

We have been seeking a formula for the summation of logarithms of $\nu$ in the Laplace transform $\tilde{g}(\nu)$ of the thrust distribution. We use Eq. (141) for $\tilde{g}(\nu)$, choosing for $\left.\mid \rho_{\mathrm{H}}\right)$ the state with a quark and an antiquark with opposite momenta. The operator $\mathcal{O}(\nu)$ acting on this state is just 1 . Then

$$
\tilde{g}(\nu)=\frac{1}{\sigma_{\mathrm{H}}}\left(1\left|\mathcal{Y}\left(Q^{2} ; \nu\right)\right| \rho_{\mathrm{H}}\right) .
$$


We approximate $\mathcal{Y}\left(Q^{2} ; \nu\right)$, using Eq. (145), as the exponential of the integral of the first order generator $\mathcal{S}_{\mathcal{Y}}^{[1]}$, which we take from Eq. (209). This gives

$$
\tilde{g}(\nu) \approx \exp \left(-\int_{\mu_{f}^{2} / Q^{2}}^{1} \frac{d y}{y}\left(1-e^{-\nu y}\right) \lambda(y)\right) .
$$

Here $\lambda(y)$ can be either the exact function from DEDUCTOR, as in Eq. (210), or else the approximate function given in Eq. (215). The factor $\left(1-e^{-\nu y}\right)$ puts an effective lower cutoff on the $y$ integration at $y=1 / \nu$. Then a factor $\log ^{n}(y)$ in $\lambda(y)$ produces a factor $\log ^{n+1}(\nu)$ in the exponent of Eq. (220).

We have seen that one can start with Eq. (136) for $\tilde{g}(\nu)$ as given by a parton shower and rearrange the operators to express $\tilde{g}(\nu)$ in the form Eq. (219). Then approximating $\mathcal{S}_{\mathcal{Y}}\left(\mu^{2} ; \nu\right)$ by $\mathcal{S}_{\mathcal{Y}}^{[1]}\left(\mu^{2} ; \nu\right)$ in $\mathcal{Y}$ gives us a candidate result (220) for the summation of logarithms of $\nu$ in $\tilde{g}(\nu)$. We do note that the shower splitting functions contain ingredients related to the argument of $\alpha_{s}$ in the parton splitting function. These ingredients are somewhat ad hoc from the perspective of just representing the soft and collinear singularities of a single splitting. Their purpose was to build into the first order splitting functions some approximation to splitting functions beyond leading order so as to improve the effectiveness of a parton shower in summing large logarithms. We have seen the effect of these ingredients in giving us the standard summation of thrust logarithms at the NLL level.

Our analysis uses primarily the Laplace transform $\tilde{g}(\nu)$ of the thrust distribution. One can take the inverse Laplace transform of $\tilde{g}(\nu)$ to obtain the thrust distribution $g(\tau)$, Eq. (125), itself. The function $g(\tau)$ is the derivative of $f(\tau)$, Eq. (127):

$$
g(\tau)=\frac{d f(\tau)}{d \tau}
$$

We can follow Ref. [35] to evaluate $f(\tau)$ at NLL accuracy:

$f(\tau)=\exp \left(-\frac{C_{\mathrm{F}}}{\pi \beta_{0}^{2}}\left\{\frac{f_{1}(\lambda)}{\alpha_{s}\left(Q^{2}\right)}+f_{2}(\lambda)\right\}\right) \frac{1}{\Gamma(1-\gamma(\lambda))}$.

Here

$$
\lambda=\beta_{0} \alpha_{s}\left(Q^{2}\right) \log (1 / \tau),
$$

the LL function $f_{1}(\lambda)$ is

$$
f_{1}(\lambda)=(1-2 \lambda) \log (1-2 \lambda)-2(1-\lambda) \log (1-\lambda),
$$

the NLL function $f_{2}(\lambda)$ is

$$
\begin{aligned}
f_{2}(\lambda)= & -\frac{\beta_{1}}{2 \beta_{0}} 2 \log ^{2}(1-\lambda)+\frac{\beta_{1}}{2 \beta_{0}} \log ^{2}(1-2 \lambda) \\
& +2 \beta_{0} \gamma_{\mathrm{E}} \log \left(\frac{1-\lambda}{1-2 \lambda}\right) \\
& -\left(\frac{\beta_{1}}{\beta_{0}}+\beta_{0} \log \left(\lambda_{\mathrm{R}}\right)\right) \log \left(\frac{(1-\lambda)^{2}}{1-2 \lambda}\right) \\
& +\frac{3 \beta_{0}}{2} \log (1-\lambda),
\end{aligned}
$$

where $\gamma_{\mathrm{E}}$ is Euler's constant, and the function $\gamma(\lambda)$ is

$$
\gamma(\lambda)=-\frac{2 C_{\mathrm{F}}}{\pi \beta_{0}} \log \left(\frac{1-\lambda}{1-2 \lambda}\right) .
$$

The logarithm of $f(\tau)$ contains LL contributions proportional to $\alpha_{s}\left(Q^{2}\right)^{n} \log ^{n+1}(1 / \tau)$ and NLL contributions proportional to $\alpha_{s}\left(Q^{2}\right)^{n} \log ^{n}(1 / \tau)$, but contributions proportional to $\alpha_{s}\left(Q^{2}\right)^{n} \log ^{j}(1 / \tau)$ with $j<n$ are dropped. Of course, a parton shower does not drop terms beyond NLL.

\section{RESULT FROM THE PARTON SHOWER}

We have manipulated the operators used in a parton shower to produce a candidate formula (220) for the summation of logarithms for the thrust distribution. We have seen that this formula reproduces the known result [35] for $\tilde{g}(\nu)$ in QCD at the NLL level. We now ask what the result for $\tilde{g}(\nu)$ is in a first order parton shower that uses the DEDUCTOR algorithm or another algorithm of interest. That is, what do we get from Eqs. (136) and (104),

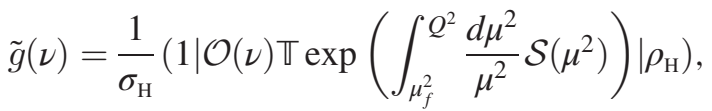

when the shower generator $\mathcal{S}\left(\mu^{2}\right)$ represents a first order shower? This must be the same as the result of using Eq. (145) in Eq. (141),

$\tilde{g}(\nu)=\frac{1}{\sigma_{\mathrm{H}}}\left(1\left|\mathbb{T} \exp \left(\int_{\mu_{f}^{2}}^{Q^{2}} \frac{d \mu^{2}}{\mu^{2}} \mathcal{S}_{\mathcal{Y}}\left(\mu^{2} ; \nu\right)\right) \mathcal{O}(\nu)\right| \rho_{\mathrm{H}}\right)$.

Here we take $\left.\mid \rho_{\mathrm{H}}\right)$ to be the initial $q \bar{q}$ state in $e^{+} e^{-}$ annihilation (with massless quarks). Then there is some simplification because $\left.\left.\mathcal{O}(\nu) \mid \rho_{\mathrm{H}}\right)=\mid \rho_{\mathrm{H}}\right)$. There is a more significant simplification because $\left.\mid \rho_{\mathrm{H}}\right)$ is an eigenvector of $\mathcal{S}_{\mathcal{Y}}\left(\mu^{2} ; \nu\right)$. We use Eq. (177), Eq. (178), and $\left(1 \mid \rho_{\mathrm{H}}\right)=\sigma_{\mathrm{H}}$ to give

$\tilde{g}(\nu)=\exp \left(\int_{\mu_{f}^{2}}^{Q^{2}} \frac{d \mu^{2}}{\mu^{2}}\left(1\left|\mathcal{S}_{\mathcal{Y}}\left(\mu^{2} ; \nu\right)\right|\{p, f, c, c\}_{2}\right)\right)$. 
Here $\left.\mid\{p, f, c, c\}_{2}\right)$ is a color singlet $q \bar{q}$ basis state with $p_{1}+p_{2}=Q$. The results are independent of the direction of $\vec{p}_{1}=-\vec{p}_{2}$ and independent of the quark flavor $f_{1}=-f_{2}$. There is only one possible color state. The basis state is normalized to $\left(1 \mid\{p, f, c, c\}_{2}\right)=1$ [19].

We use the operator $\mathcal{I}(\nu)$ defined in Eq. (146),

$$
\mathcal{I}(\nu)=\int_{\mu_{f}^{2}}^{Q^{2}} \frac{d \mu^{2}}{\mu^{2}} \mathcal{S}_{\mathcal{Y}}\left(\mu^{2} ; \nu\right),
$$

to write Eq. (229) as

$$
\tilde{g}(\nu)=\exp \left[\left(1|\mathcal{I}(\nu)|\{p, f, c, c\}_{2}\right)\right] .
$$

In Eq. (231), $\mathcal{I}(\nu)$ is obtained from just $\mathcal{S}\left(\mu^{2}\right)$, not from any higher order splitting functions that might be present in a higher order shower algorithm. The result for $\tilde{g}(\nu)$ in Eq. (231) could be very different from $\tilde{g}(\nu)$ as given by Eq. (220) because a first order parton shower is not the same as full QCD.

Using Eq. (169), we expand $\mathcal{I}(\nu)$ as a series of terms $\mathcal{I}^{[k]}(\nu)$, where $\mathcal{I}^{[k]}(\nu)$ contains $k$ powers of the shower splitting operator $\mathcal{S}\left(\mu^{2}\right)$. Thus $\mathcal{I}^{[k]}(\nu)$ contains $k$ powers of $\alpha_{\mathrm{s}}$ evaluated at a running scale inside the integrations that give $\mathcal{I}^{[k]}(\nu)$. We can expand $\mathcal{I}^{[k]}(\nu)$ in powers of $\alpha_{s}$ evaluated at a fixed scale. A convenient choice $^{9}$ is $\mu_{\text {fixed }}^{2}=Q^{2} / \nu$. Thus we write

$$
\mathcal{I}^{[k]}(\nu)=\sum_{n=k}^{\infty}\left[\frac{\alpha_{s}\left(Q^{2} / \nu\right)}{2 \pi}\right]^{n} \mathcal{I}_{n}^{[k]}(\nu)
$$

In $\mathcal{I}_{k}^{[k]}(\nu)$ there are $k$ integrations over scale variables $y$ and $k$ integrations over momentum fractions $z$, so $\mathcal{I}_{k}^{[k]}(\nu)$ could contain $2 k$ factors of $\log (\nu)$. Changing the scale in $\alpha_{s}$ can produce one more factor $\log (\nu)$ for each factor $\alpha_{s}$, so that $\mathcal{I}_{n}^{[k]}(\nu)$ could contain $n+k$ factors of $\log (\nu)$. However the exponent in $\tilde{g}(\nu)$ in Eq. (220) contains only contributions proportional to $\alpha_{s}^{n}\left(Q^{2} / \nu\right) \log ^{j}(\nu)$ with $j \leq n+1$. Thus a minimal expectation for the parton shower is that $\mathcal{I}_{n}^{[k]}(\nu)$ contains only $j$ factors of $\log (\nu)$ with $j \leq n+1$. If this is the case, we can say that the $\log (\nu)$ factors exponentiate.

If we expand the QCD result for the exponent in $\tilde{g}(\nu)$ as given by Eq. (220) in powers of $\alpha_{s}\left(Q^{2} / \nu\right)$, the coefficients of $\alpha_{s}^{n}\left(Q^{2} / \nu\right) \log ^{n+1}(\nu)$ and $\alpha_{s}^{n}\left(Q^{2} / \nu\right) \log ^{n}(\nu)$ take particular

\footnotetext{
${ }^{9}$ In Sec. XIX, we used $\mu_{\text {fixed }}^{2}=Q^{2}$. Using Eq. (216), one can transform between expansions $\sum c(n, j) \alpha_{s}^{n}\left(Q^{2} / \nu\right) \log ^{j}(\nu)$ and $\sum c^{\prime}(n, j) \alpha_{s}^{n}\left(Q^{2}\right) \log ^{j}(\nu)$ with $j \leq n$ in each case, so both choices of $\mu_{\text {fixed }}^{2}$ work equally well in an analytical treatment. In a numerical evaluation, $\mu_{\text {fixed }}^{2}=Q^{2} / \nu$ has the advantage that this scale is closer to the running scale at which $\alpha_{s}$ is evaluated inside the integrals for $\mathcal{I}^{[k]}(\nu)$.
}

values. These values are generated by $\mathcal{I}^{[1]}(\nu)$ using $\alpha_{s}$ with its argument suitably specified by the shower algorithm. Thus for $k \geq 2, \mathcal{I}_{n}^{[k]}(\nu)$ must not contain a factor $\log ^{n+1}(\nu)$ if we are to maintain the logarithmic summation at LL level and additionally must not contain a factor $\log ^{n}(\nu)$ if we are to maintain the logarithmic summation at NLL level.

We investigate how many powers of $\log (\nu)$ are contained in $\mathcal{I}_{n}^{[k]}(\nu)$ in the following two sections.

\section{PARTON SHOWER AT LEADING LOG}

In this section we examine the operators $\mathcal{S}_{\mathcal{Y}}^{[k]}\left(\mu^{2} ; \nu\right)$ with the aim of discovering the behavior of $\tilde{g}(\nu)$ as given by a leading order parton shower using the $\Lambda$-ordered DEDUCTOR algorithm with exact QCD color. The Laplace transform of $\tilde{g}(\nu)$ can be represented according to Eq. (231) in terms of the integral $\mathcal{I}(\nu)$ of $\mathcal{S}_{\mathcal{Y}}\left(\mu^{2} ; \nu\right)$ defined in Eq. (146). We write the definition in the form

$$
\mathcal{I}(\nu)=\int_{0}^{\nu} \frac{d x}{x} \mathcal{S}_{\mathcal{Y}}\left(x Q^{2} / \nu ; \nu\right) .
$$

Here we have defined a standard scale $Q^{2} / \nu$ and a scale variable $x$ that gives the ratio of $\mu^{2}$ to this standard scale: $\mu^{2}=x Q^{2} / \nu$. If we expand the exponential (not just the exponent) in Eq. (231) in powers of $\alpha_{\mathrm{s}}\left(Q^{2} / \nu\right)$, we will find terms proportional to $\alpha_{s}^{n}\left(Q^{2} / \nu\right) \log ^{j}(\nu)$ with $j \leq 2 n$.

The simplest expectation would be that $\mathcal{I}(\nu)$ also has an expansion with terms $\alpha_{s}^{n}\left(Q^{2} / \nu\right) \log ^{j}(\nu)$ with $j \leq 2 n$. Such a representation would not be very useful, even if we knew all of the coefficients for $j=2 n$. It is much more useful if there are nonzero contributions $\alpha_{s}^{n}\left(Q^{2} / \nu\right) \log ^{j}(\nu)$ only for $j \leq n+1$ and we knew the coefficients for terms with $j=n+1$. We then call the $j=n+1$ terms the leading $\log$, LL, terms.

In the notation of this paper, the operator $\mathcal{I}^{[1]}(\nu)$ is proportional to one power of the shower splitting operator and thus to one power of a running $\alpha_{s}$ rather than the fixed $\alpha_{s}\left(Q^{2} / \nu\right)$. As we have seen, this operator generates a whole LL series $\alpha_{s}^{n}\left(Q^{2} / \nu\right) \log ^{j}(\nu)$ with $j=n+1$. We may hope that this is all that survives at the LL level. That is, we may hope that $\mathcal{I}^{[k]}(\nu)$ for $k \geq 2$ generates only terms $\alpha_{s}^{n}\left(Q^{2} / \nu\right) \log ^{j}(\nu)$ with $j \leq n$. If so, we will say that $\tilde{g}(\nu)$ as given by the leading order parton shower exponentiates at the LL level.

In this section, we demonstrate that $\tilde{g}(\nu)$ does exponentiate at the LL level in this sense. In the following section, we will turn our attention to the NLL level.

We will need a small preliminary analysis. We see from Eq. (175) that for $\mathcal{S}_{\mathcal{Y}}^{[2]}\left(x Q^{2} / \nu ; \nu\right)$ we will need $\left[\mathcal{O}(\nu) \mathcal{S}\left(\bar{\mu}^{2}\right) \mathcal{O}^{-1}(\nu)\right]_{\mathbb{P}}$ and $\left[\mathcal{O}(\nu) \mathcal{S}\left(\bar{\mu}^{2}\right) \mathcal{O}^{-1}(\nu)\right]_{1-\mathbb{P}}$.

For $\left[\mathcal{O}(\nu) \mathcal{S}\left(\bar{\mu}^{2}\right) \mathcal{O}^{-1}(\nu)\right]_{\mathbb{P}}$, we briefly repeat the derivation that gave us Eq. (208). We use Eq. (107), then Eq. (181), then Eqs. (184) and (207): 


$$
\begin{aligned}
{[\mathcal{O}(\nu)} & \left.\mathcal{S}\left(x Q^{2} / \nu\right) \mathcal{O}^{-1}(\nu)\right]_{\mathbb{P}} \\
= & {\left[\mathcal{O}(\nu) \mathcal{S}^{[1,0]}\left(x Q^{2} / \nu\right) \mathcal{O}^{-1}(\nu)+\mathcal{S}^{[0,1]}\left(x Q^{2} / \nu\right)\right]_{\mathbb{P}} } \\
= & {\left[\mathcal{O}(\nu) \mathcal{S}^{[1,0]}\left(x Q^{2} / \nu\right) \mathcal{O}^{-1}(\nu)-\mathcal{S}^{[1,0]}\left(x Q^{2} / \nu\right)\right]_{\mathbb{P}} } \\
= & \sum_{l}\left[\mathcal{S}_{l}^{[1,0]}\left(x Q^{2} / \nu\right) e^{-\xi_{l}^{\mathrm{op}} x}-\mathcal{S}_{l}^{[1,0]}\left(x Q^{2} / \nu\right)\right]_{\mathbb{P}} \\
= & -\sum_{l}\left[\mathcal{S}_{l}^{[1,0]}\left(x Q^{2} / \nu\right)\right]_{\mathbb{P}}\left(1-e^{-\xi_{l}^{\text {op }} x}\right) .
\end{aligned}
$$

For $\left[\mathcal{O}(\nu) \mathcal{S}\left(\bar{\mu}^{2}\right) \mathcal{O}^{-1}(\nu)\right]_{1-\mathbb{P}}$, we need a somewhat different argument. We use Eqs. (176) and (107). Then we note that $\left[\mathcal{S}^{[0,1]}\left(x Q^{2} / \nu\right)\right]_{\mathbb{P}}=\mathcal{S}^{[0,1]}\left(x Q^{2} / \nu\right)$ according to Eq. (162) because $\mathcal{S}^{[0,1]}\left(x Q^{2} / \nu\right)$ leaves the parton momenta and flavors unchanged. Then we use Eqs. (184) and (207). Finally, we use the definition (176) again. This gives

$$
\begin{aligned}
{\left[\mathcal{O}(\nu) \mathcal{S}\left(x Q^{2} / \nu\right) \mathcal{O}^{-1}(\nu)\right]_{1-\mathbb{P}} } & =\left\{\mathcal{O}(\nu) \mathcal{S}^{[1,0]}\left(x Q^{2} / \nu\right) \mathcal{O}^{-1}(\nu)+\mathcal{S}^{[0,1]}\left(x Q^{2} / \nu\right)-\left[\mathcal{O}(\nu) \mathcal{S}^{[1,0]}\left(x Q^{2} / \nu\right) \mathcal{O}^{-1}(\nu)+\mathcal{S}^{[0,1]}\left(x Q^{2} / \nu\right)\right]_{\mathbb{P}}\right\} \\
& =\left\{\mathcal{O}(\nu) \mathcal{S}^{[1,0]}\left(x Q^{2} / \nu\right) \mathcal{O}^{-1}(\nu)-\left[\mathcal{O}(\nu) \mathcal{S}^{[1,0]}\left(x Q^{2} / \nu\right) \mathcal{O}^{-1}(\nu)\right]_{\mathbb{P}}\right\} \\
& =\left\{\sum_{l} \mathcal{S}_{l}^{[1,0]}\left(x Q^{2} / \nu\right) e^{-\xi_{l}^{\mathrm{op}} x}-\sum_{l}\left[\mathcal{S}_{l}^{[1,0]}\left(x Q^{2} / \nu\right)\right]_{\mathbb{P}} e^{-\xi_{l}^{\mathrm{op}} x}\right\} \\
& =\sum_{l}\left[\mathcal{S}_{l}^{[1,0]}\left(x Q^{2} / \nu\right)\right]_{1-\mathbb{P}} e^{-\xi_{l}^{\mathrm{op}} x} .
\end{aligned}
$$

Now we can start with $\mathcal{S}_{\mathcal{Y}}\left(x Q^{2} / \nu ; \nu\right)$ at first order. Equation (208) gives us the result on the right-hand side of Eq. (234):

$\mathcal{S}_{\mathcal{Y}}^{[1]}\left(x Q^{2} / \nu ; \nu\right) \approx-\sum_{l}\left[\mathcal{S}_{l}^{[1,0]}\left(x Q^{2} / \nu\right)\right]_{\mathbb{P}}\left(1-e^{-\xi_{l}^{\mathrm{op}} x}\right)$.

Recall that the eigenvalue $\xi_{l}$ of $\xi_{l}^{\text {op }}$, given by Eq. (204), is of order 1 . We will also need $\mathcal{Y}^{[1]}\left(x Q^{2} / \nu ; \nu\right)$. When we substitute Eq. (236) into Eq. (174), we obtain

$\mathcal{Y}^{[1]}\left(x Q^{2} / \nu ; \nu\right)=-\int_{0}^{x} \frac{d \bar{x}}{\bar{x}} \sum_{l}\left[\mathcal{S}_{l}^{[1,0]}\left(\bar{x} Q^{2} / \nu\right)\right]_{\mathbb{P}}\left(1-e^{-\xi_{l}^{\mathrm{op}} \bar{x}}\right)$.

Here, and in the remainder of this section, we set the infrared cutoff $\mu_{f}^{2}$ to zero. We notice that the factor $\left(1-e^{-\xi_{l} \bar{x}}\right)$ is small for $\bar{x} \ll 1$ and approaches zero like $\bar{x}$ when $\bar{x} \rightarrow 0$. This provides an infrared cutoff for the $\bar{x}$ integration.

Now look at $\mathcal{S}_{\mathcal{Y}}\left(x Q^{2} / \nu ; \nu\right)$ at second order. We use Eq. (175):

$$
\begin{aligned}
\mathcal{S}_{\mathcal{Y}}^{[2]}\left(x Q^{2} / \nu ; \nu\right)= & \int_{0}^{x} \frac{d \bar{x}}{\bar{x}}\left[\left[\mathcal{O}(\nu) \mathcal{S}\left(\bar{x} Q^{2} / \nu\right) \mathcal{O}^{-1}(\nu)\right]_{\mathbb{P}}\right. \\
& \left.\times\left[\mathcal{O}(\nu) \mathcal{S}\left(x Q^{2} / \nu\right) \mathcal{O}^{-1}(\nu)\right]_{1-\mathbb{P}}\right]_{\mathbb{P}} .
\end{aligned}
$$

With the results (234) and (235), we obtain

$$
\begin{aligned}
\mathcal{S}_{\mathcal{Y}}^{[2]}\left(x Q^{2} / \nu ; \nu\right)= & -\sum_{\bar{l}, l} \int_{0}^{x} \frac{d \bar{x}}{\bar{x}}\left[\left[\mathcal{S}_{\bar{l}}^{[1,0]}\left(\bar{x} Q^{2} / \nu\right)\right]_{\mathbb{P}}\left(1-e^{-\xi_{\bar{l}}^{\mathrm{op} \bar{x}}}\right)\right. \\
& \left.\times\left[\mathcal{S}_{l}^{[1,0]}\left(x Q^{2} / \nu\right)\right]_{1-\mathbb{P}} e^{-\xi_{l}^{\mathrm{op}} x}\right]_{\mathbb{P}}
\end{aligned}
$$

We integrate this to form the contribution to $\mathcal{I}$, Eq. (146), with two powers of $\mathcal{S}$ :

$$
\mathcal{I}^{[2]}(\nu)=\int_{0}^{\nu} \frac{d x}{x} \mathcal{S}_{\mathcal{Y}}^{[2]}\left(x Q^{2} / \nu ; \nu\right) .
$$

There are potentially two $\log (\nu)$ factors from the $z$ integrations inside the two factors of $\mathcal{S}_{l}^{[1,0]}$. After expanding the running couplings in $\mathcal{S}_{l}^{[1,0]}$, at order $\alpha_{s}^{n}\left(Q^{2} / \nu\right)$ there could be a total of $n$ factors of $\log (\nu)$. Then we integrate over $x$ and $\bar{x}$. This could produce two more factors of $\log (\nu)$, giving $\log ^{n+2}(\nu)$ at order $\alpha_{s}^{n}\left(Q^{2} / \nu\right)$. But what happens in the $x$ and $\bar{x}$ integrations that we find based on Eq. (239)? If $\bar{x} \ll 1$, the factor $\left(1-e^{-\xi_{\bar{F}} \bar{x}}\right)$ is small, so that the $\bar{x}$ integration is effectively limited to the range $1 \lesssim \bar{x}$. If $1 \ll x$, the factor $e^{-\xi_{i} x}$ is small, so that the $x$ integration is effectively limited to the range $x \lesssim 1$. We also have $\bar{x}<x$. Thus the net effective integration range is $1 \lesssim \bar{x}<x \lesssim 1$. This leaves only $\bar{x} \sim x \sim 1$. There are no $\log (\nu)$ factors from the $\bar{x}$ and $x$ integrations.

A contribution to $\mathcal{I}^{[2]}$ proportional to $\alpha_{s}^{n}\left(Q^{2} / \nu\right) \log ^{n+1}(\nu)$ can be designated leading log. The result (239) shows that there is no LL contribution to $\mathcal{I}^{[2]}$. Rather, the LL contributions to the integral $\mathcal{I}$ of $\mathcal{S}_{\mathcal{Y}}$ come from $\mathcal{S}_{\mathcal{Y}}^{[1]}$ after we account for the argument of the strong coupling $\alpha_{s}$ in $\mathcal{S}_{\mathcal{Y}}^{[1]}$, Eq. (218). This leaves the possibility of a NLL, $\alpha_{s}^{n}\left(Q^{2} / \nu\right) \log ^{n}(\nu)$, contribution to $\mathcal{I}^{[2]}$. We will investigate 
the NLL contribution in the following section by looking at the $z$ integrations in $\mathcal{S}_{\mathcal{Y}}^{[2]}$.

We will also need some qualitative information about the behavior of $\mathcal{Y}^{[2]}$. From Eq. (171) we have

$$
\begin{aligned}
\mathcal{Y}^{[2]}\left(x Q^{2} / \nu ; \nu\right)= & \int_{0}^{x} \frac{d \bar{x}}{\bar{x}}\left\{\mathcal{S}_{\mathcal{Y}}^{[2]}\left(\bar{x} Q^{2} / \nu ; \nu\right)\right. \\
& \left.+\mathcal{Y}^{[1]}\left(\bar{x} Q^{2} / \nu ; \nu\right) \mathcal{S}_{\mathcal{Y}}^{[1]}\left(\bar{x} Q^{2} / \nu ; \nu\right)\right\} .
\end{aligned}
$$

Using Eqs. (239), (236), and (237),

$$
\begin{aligned}
\mathcal{Y}^{[2]}\left(x Q^{2} / \nu ; \nu\right)= & \sum_{l_{1}, l_{2}} \int_{0}^{x} \frac{d x_{1}}{x_{1}} \int_{0}^{x_{1}} \frac{d x_{2}}{x_{2}} \\
& \times\left\{\left[\left[\mathcal{S}_{l_{2}}^{[1,0]}\left(x_{2} Q^{2} / \nu\right)\right]_{\mathbb{P}}\left(1-e^{-\xi_{l_{2}}^{\mathrm{op}} x_{2}}\right)\right.\right. \\
& \left.\times\left[\mathcal{S}_{l_{1}}^{[1,0]}\left(x_{1} Q^{2} / \nu\right)\right]_{1-\mathbb{P}} e^{-\xi_{l_{1}}^{\mathrm{op}} x_{1}}\right]_{\mathbb{P}} \\
& +\left[\mathcal{S}_{l_{2}}^{[1,0]}\left(x_{2} Q^{2} / \nu\right)\right]_{\mathbb{P}}\left(1-e^{-\xi_{l_{2}}^{\mathrm{op}} x_{2}}\right) \\
& \left.\times\left[\mathcal{S}_{l_{1}}^{[1,0]}\left(x_{1} Q^{2} / \nu\right)\right]_{\mathbb{P}}\left(1-e^{-\xi_{l_{1}}^{\mathrm{op}} x_{1}}\right)\right\} .
\end{aligned}
$$

In both terms we have a factor $\left(1-e^{-\xi_{1} x_{2}}\right)$ so there is an effective integration range $1 \lesssim x_{2}<x_{1}<x$. This implies that $\mathcal{Y}^{[2]}\left(x Q^{2} / \nu ; \nu\right) \rightarrow 0$ for $x \ll 1$. In the first term, there is a factor $e^{-\xi_{1} x_{1}}$, so that the integrand is small for $1 \ll x_{1}$. However the second term contains no such factor. The operators $\mathcal{S}_{l_{1}}^{[1,0]}\left(x_{1} Q^{2} / \nu\right)$ and $\mathcal{S}_{l_{2}}^{[1,0]}\left(x_{2} Q^{2} / \nu\right)$ can give us logarithms of their arguments. For this reason, $\mathcal{Y}^{[2]}\left(x Q^{2} / \nu ; \nu\right)$ can grow slowly, like a power of $\log (x)$, for $1 \ll x$.

If we take $x=1$ in Eq. (242), the effective integration range for $x_{1}$ and $x_{2}$ is $1 \lesssim x_{2}<x_{1}<1$. Thus $x_{2} \sim x_{1} \sim 1$. Then there are no factors of $\log (\nu)$ produced by the integrations over $x_{1}$ and $x_{2}$. Each factor of $\mathcal{S}_{l}^{[1,0]}\left(Q^{2} / \nu\right)$ contains one factor of $\log (\nu)$. Thus $\mathcal{Y}^{[2]}\left(Q^{2} / \nu ; \nu\right)$ contains at most 2 factors of $\log (\nu)$.

We can generalize these observations to suggest induction hypotheses for $\mathcal{S}_{\mathcal{Y}}^{[k]}$ and $\mathcal{Y}^{[k]}$ for $k \geq 2$ :

(1) The operator $\mathcal{S}_{\mathcal{Y}}^{[k]}\left(x Q^{2} / \nu ; \nu\right)$ is suppressed by a factor $x$ times logarithms for $x \rightarrow 0$ and by an exponential $e^{-c x}$ times logarithms for $x \rightarrow \infty$. Its only unsuppressed region is for $x \sim 1$.

(2) The operator $\mathcal{Y}^{[k]}\left(x Q^{2} / \nu ; \nu\right)$ is suppressed by a factor $x$ times logarithms for $x \rightarrow 0$ and grows at most logarithmically for $x \rightarrow \infty$.

(3) The operators $\mathcal{S}_{\mathcal{Y}}^{[k]}\left(Q^{2} / \nu ; \nu\right)$ and $\mathcal{Y}^{[k]}\left(Q^{2} / \nu ; \nu\right)$ each contain at most $k$ factors of $\log (\nu)$ at order $\alpha_{s}^{k}\left(Q^{2} / \nu\right)$.

In property 3 , we note that the operators $\mathcal{S}_{\mathcal{Y}}^{[k]}\left(Q^{2} / \nu ; \nu\right)$ and $\mathcal{Y}^{[k]}\left(Q^{2} / \nu ; \nu\right)$ contain higher powers of $\alpha_{s}\left(Q^{2} / \nu\right)$ that arise from expanding the running couplings in their definitions in powers of $\alpha_{s}\left(Q^{2} / \nu\right)$. This expansion can yield one more power of $\log (\nu)$ per power of $\alpha_{s}\left(Q^{2} / \nu\right)$. Thus there are at most $n$ powers of $\log (\nu)$ at order $\alpha_{s}^{n}\left(Q^{2} / \nu\right)$.

We have found that these properties hold at order $k=2$. We now establish that they hold for any larger order by assuming that they hold at order $k$ and showing that they hold at order $k+1$.

Begin with $\mathcal{S}_{\mathcal{Y}}^{[k+1]}$. From Eq. (170) we have

$$
\begin{aligned}
\mathcal{S}_{\mathcal{Y}}^{[k+1]} & \left(x Q^{2} / \nu ; \nu\right) \\
= & {\left[\mathcal{Y}^{[k]}\left(x Q^{2} / \nu ; \nu\right)\right.} \\
& \left.\times\left\{\mathcal{O}(\nu) \mathcal{S}\left(x Q^{2} / \nu\right) \mathcal{O}(\nu)-\mathcal{S}_{\mathcal{Y}}^{[1]}\left(x Q^{2} / \nu ; \nu\right)\right\}\right]_{\mathbb{P}} \\
& -\sum_{j=2}^{k-1}\left[\mathcal{Y}[k+1-j]\left(x Q^{2} / \nu ; \nu\right) \mathcal{S}_{\mathcal{Y}}^{[j]}\left(x Q^{2} / \nu ; \nu\right)\right]_{\mathbb{P}} \\
& -\left[\mathcal{Y}^{[1]}\left(x Q^{2} / \nu ; \nu\right) \mathcal{S}_{\mathcal{Y}}^{[k]}\left(x Q^{2} / \nu ; \nu\right)\right]_{\mathbb{P}} .
\end{aligned}
$$

We use Eq. (172) to simplify the first term and Eqs. (174) and (172) to simplify the last term:

$$
\begin{aligned}
\mathcal{S}_{\mathcal{Y}}^{[k+1]} & \left(x Q^{2} / \nu ; \nu\right) \\
= & {\left[\mathcal{Y}^{[k]}\left(x Q^{2} / \nu ; \nu\right)\left[\mathcal{O}(\nu) \mathcal{S}\left(x Q^{2} / \nu\right) \mathcal{O}(\nu)\right]_{1-\mathbb{P}}\right]_{\mathbb{P}} } \\
& -\sum_{j=2}^{k-1}\left[\mathcal{Y}[k+1-j]\left(x Q^{2} / \nu ; \nu\right) \mathcal{S}_{\mathcal{Y}}^{[j]}\left(x Q^{2} / \nu ; \nu\right)\right]_{\mathbb{P}} \\
& -\left[\int_{0}^{x} \frac{d \bar{x}}{\bar{x}}\left[\mathcal{O}(\nu) \mathcal{S}\left(\bar{x} Q^{2} / \nu\right) \mathcal{O}(\nu)\right]_{\mathbb{P}}\right. \\
& \left.\times \mathcal{S}_{\mathcal{Y}}^{[k]}\left(x Q^{2} / \nu ; \nu\right)\right]_{\mathbb{P}}
\end{aligned}
$$

Now we can use Eq. (235) in the first term and Eq. (234) in the last term, giving us

$$
\begin{aligned}
\mathcal{S}_{\mathcal{Y}}^{[k+1]} & \left(x Q^{2} / \nu ; \nu\right) \\
= & \sum_{l}\left[\mathcal{Y}^{[k]}\left(x Q^{2} / \nu ; \nu\right)\left[\mathcal{S}_{l}^{[1,0]}\left(x Q^{2} / \nu\right)\right]_{1-\mathbb{P}}\right]_{\mathbb{P}} e^{-\xi_{l}^{\mathrm{op}} x} \\
& -\sum_{j=2}^{k-1}\left[\mathcal{Y}^{[k+1-j]}\left(x Q^{2} / \nu ; \nu\right) \mathcal{S}_{\mathcal{Y}}^{[j]}\left(x Q^{2} / \nu ; \nu\right)\right]_{\mathbb{P}} \\
& +\sum_{l}\left[\int_{0}^{x} \frac{d \bar{x}}{\bar{x}}\left[\mathcal{S}_{l}^{[1,0]}\left(\bar{x} Q^{2} / \nu\right)\right]_{\mathbb{P}}\left(1-e^{-\xi_{l}^{\mathrm{op}} \bar{x}}\right)\right. \\
& \left.\times \mathcal{S}_{\mathcal{Y}}^{[k]}\left(x Q^{2} / \nu ; \nu\right)\right]_{\mathbb{P}} .
\end{aligned}
$$

In the first term, property 2 for $\mathcal{Y}^{[k]}\left(x Q^{2} / \nu ; \nu\right)$ implies that this term is unsuppressed only for $1 \lesssim x$, while the factor 
$\exp \left(-\xi_{l}^{\mathrm{op}} x\right)$ implies that this term is unsuppressed only for $x \lesssim 1$. Thus this term is unsuppressed only for $x \sim 1$. In the second term, property 1 for $\mathcal{S}_{\mathcal{Y}}^{[j]}\left(x Q^{2} / \nu ; \nu\right)$ implies that this term is unsuppressed only for $x \sim 1$. In the third term, property 1 for $\mathcal{S}_{\mathcal{Y}}^{[k]}\left(x Q^{2} / \nu ; \nu\right)$ implies that this term is unsuppressed only for $x \sim 1$. This gives us property 1 for $\mathcal{S}_{\mathcal{Y}}^{[k+1]}\left(x Q^{2} / \nu ; \nu\right)$.

Now set $x=1$ in Eq. (245). There is an integration over $\bar{x}$ in the third term, but, accounting for the factor $\left[1-\exp \left(-\xi_{l}^{\mathrm{op}} \bar{x}\right)\right]$, the integration region is $1 \lesssim \bar{x}<1$. That is, $\bar{x} \sim 1$. We can then use property 3 for the operators that appear in order to count the maximum possible number of factors of $\log (\nu)$ in each term. At order $\alpha_{s}^{k+1}$, this gives the maximum number of factors of $\log (\nu)$ as $k+1$, thus verifying property 3 for $\mathcal{S}_{\mathcal{Y}}^{[k+1]}\left(Q^{2} / \nu ; \nu\right)$.

Now we examine $\mathcal{Y}\left(x Q^{2} / \nu ; \nu\right)$. We use Eq. (171) to write for $k \geq 1$,

$$
\begin{aligned}
\mathcal{Y}^{[k+1]} & \left(x Q^{2} / \nu ; \nu\right) \\
= & \int_{0}^{x} \frac{d \bar{x}}{\bar{x}} \mathcal{Y}^{[k]}\left(\bar{x} Q^{2} / \nu ; \nu\right) \mathcal{S}_{\mathcal{Y}}^{[1]}\left(\bar{x} Q^{2} / \nu ; \nu\right) \\
& +\sum_{j=2}^{k} \int_{0}^{x} \frac{d \bar{x}}{\bar{x}} \mathcal{Y}^{[k+1-j]}\left(\bar{x} Q^{2} / \nu ; \nu\right) \mathcal{S}_{\mathcal{Y}}^{[j]}\left(\bar{x} Q^{2} / \nu ; \nu\right) \\
& +\int_{0}^{x} \frac{d \bar{x}}{\bar{x}} \mathcal{S}_{\mathcal{Y}}^{[k+1]}\left(\bar{x} Q^{2} / \nu ; \nu\right) .
\end{aligned}
$$

We use Eq. (172) and (234) to simplify the first term:

$$
\begin{aligned}
\mathcal{Y}^{[k+1]} & \left(x Q^{2} / \nu ; \nu\right) \\
= & -\sum_{l} \int_{0}^{x} \frac{d x_{1}}{x_{1}} \mathcal{Y}^{[k]}\left(x_{1} Q^{2} / \nu ; \nu\right)\left[\mathcal{S}_{l}^{[1,0]}\left(x_{1} Q^{2} / \nu\right)\right]_{\mathbb{P}} \\
& \times\left(1-e^{-\xi_{l}^{\text {op }} x_{1}}\right) \\
& +\sum_{j=2}^{k} \int_{0}^{x} \frac{d x_{1}}{x_{1}} \mathcal{Y}^{[k+1-j]}\left(x_{1} Q^{2} / \nu ; \nu\right) \mathcal{S}_{\mathcal{Y}}^{[j]}\left(x_{1} Q^{2} / \nu ; \nu\right) \\
& +\int_{0}^{x} \frac{d x_{1}}{x_{1}} \mathcal{S}_{\mathcal{Y}}^{[k+1]}\left(x_{1} Q^{2} / \nu ; \nu\right) .
\end{aligned}
$$

In each term, condition 2 for $\mathcal{Y}^{[k]}\left(x_{1} Q^{2} / \nu ; \nu\right)$ or $\mathcal{Y}^{[k+1-j]}\left(x_{1} Q^{2} / \nu ; \nu\right)$ or condition 1 for $\mathcal{S}_{\mathcal{Y}}^{[k+1]}\left(x_{1} Q^{2} / \nu ; \nu\right)$ implies that the integrand of the $x_{1}$ integration is unsuppressed only for $1 \lesssim x_{1}$. Since $x_{1}<x, \mathcal{Y}^{[k+1]}\left(x Q^{2} / \nu ; \nu\right)$ is unsuppressed only for $1 \lesssim x$. This establishes property 2 for $\mathcal{Y}^{[k+1]}\left(x Q^{2} / \nu ; \nu\right)$.

Now set $x=1$ in Eq. (247). There is an integration over $x_{1}$ in each term, but the integration region is $1 \lesssim x_{1}<1$. We can then use property 3 for the operators that appear in order to count the maximum possible number of factors of $\log (\nu)$ in each term. At order $\alpha_{s}^{k+1}\left(Q^{2} / \nu\right)$, this gives the maximum number of factors of $\log (\nu)$ as $k+1$, thus verifying property 3 for $\mathcal{Y}^{[k+1]}\left(Q^{2} / \nu ; \nu\right)$.

We call the properties 1,2 , and 3 above the $L L$ exponentiation property of $\mathcal{S}_{\mathcal{Y}}\left(\mu^{2} ; \nu\right)$, as discussed at the start of this section. In the following section we analyze the NLL contributions to $\mathcal{S}_{\mathcal{Y}}\left(\mu^{2} ; \nu\right)$.

\section{PARTON SHOWER AT NEXT-TO-LEADING LOG}

We have seen that $\mathcal{S}_{\mathcal{Y}}\left(\mu^{2} ; \nu\right)$ has the proper perturbative structure to allow $\tilde{g}(\nu)$ as given by a leading order parton shower using the $\Lambda$-ordered DEDUCTOR algorithm to exponentiate correctly at the leading log level.

First, the operator $\mathcal{S}_{\mathcal{Y}}^{[1]}\left(\mu^{2} ; \nu\right)$, constructed from one power of the shower splitting operator $\mathcal{S}\left(\mu^{2}\right)$ has the right structure to reproduce the known QCD result [35] at LL accuracy and even at NLL accuracy, provided that the argument the running coupling $\alpha_{s}$ in $\mathcal{S}\left(\mu^{2}\right)$ is properly defined. For $\mathcal{I}^{[1]}(\nu)$, we can state this in terms of an expansion in powers of $\alpha_{s}\left(Q^{2} / \nu\right)$. We consider the integral $\mathcal{I}^{[1]}(\nu)$ of $\mathcal{S}_{\mathcal{Y}}^{[1]}\left(\mu^{2} ; \nu\right)$ defined in Eq. (233). When the running $\alpha_{s}$ in $\mathcal{I}^{[1]}(\nu)$ is expanded in powers of $\alpha_{s}\left(Q^{2} / \nu\right)$, the coefficients of $\alpha_{s}^{n}\left(Q^{2} / \nu\right) \log ^{n+1}(\nu)$, that is the LL coefficients, are correct and the coefficients of $\alpha_{s}^{n}\left(Q^{2} / \nu\right) \log ^{n}(\nu)$, the NLL coefficients, are also correct.

Second, each of the operators $\mathcal{S}_{\mathcal{Y}}^{[k]}\left(\mu^{2} ; \nu\right)$ for $k \geq 2$ has the right structure so that in the integral $\mathcal{I}^{[k]}(\nu)$, the coefficient of $\alpha_{s}^{n}\left(Q^{2} / \nu\right) \log ^{n+1}(\nu)$, which contributes to the exponent in $\tilde{g}(\nu)$ at LL accuracy, vanish. That is, the coefficient $\mathcal{I}_{n}^{[k]}(\nu)$ of $\alpha_{s}^{n}\left(Q^{2} / \nu\right)$ in $\mathcal{I}^{[k]}(\nu)$ contains at most $n$ powers of $\log (\nu)$.

This LL exponentiation property arises from two features of $\mathcal{S}_{\mathcal{Y}}^{[k]}\left(\mu^{2} ; \nu\right)$. First, $\mathcal{S}_{\mathcal{Y}}^{[k]}\left(\mu^{2} ; \nu\right)$ is suppressed for $\mu^{2} \gg Q^{2} / \nu$ and for $\mu^{2} \ll Q^{2} / \nu$, so that only the integration region $\mu^{2} \sim Q^{2} / \nu$ contributes to $\mathcal{I}^{[k]}(\nu)$ and no factor of $\log (\nu)$ arises from integrating over $\mu^{2}$ from $Q^{2} / \nu$ to $Q^{2}$. Second, $\mathcal{S}_{\mathcal{Y}}^{[k]}\left(Q^{2} / \nu ; \nu\right)$ at order $\alpha_{s}^{n}\left(Q^{2} / \nu\right)$ contains at most $n$ factors of $\log (\nu)$.

Now, if the coefficients of $\alpha_{s}^{n}\left(Q^{2} / \nu\right) \log ^{n}(\nu)$ in $\mathcal{I}^{[k]}(\nu)$ were to vanish for $k \geq 2$, then $\mathcal{I}^{[k]}(\nu)$ would not contribute to $\tilde{g}(\nu)$ at NLL level. Then the only NLL contributions to $\tilde{g}(\nu)$ would come from the expansion of the running coupling in $\mathcal{I}^{[1]}(\nu)$. Since these contributions match the known QCD result [35], we would conclude that the first order parton shower according to the DEDUCTOR algorithm generates the known QCD result at NLL accuracy.

Remarkably, this is the case: in $\mathcal{I}^{[k]}(\nu)$ for $k \geq 2$ the coefficients $\mathcal{I}_{n}^{[k]}(\nu)$ of $\alpha_{s}^{n}\left(Q^{2} / \nu\right)$ contain at most $n-1$ powers of $\log (\nu)$ for large $\nu$. The proof of this result, with exact color, is somewhat involved, so we present it in Appendix A. 


\section{NUMERICAL BEHAVIOR OF $\mathcal{I}^{[2]}(\nu)$}

We have considered analytically the coefficient $\mathcal{I}_{n}^{[k]}(\nu)$ of $\left[\alpha_{s}\left(Q^{2} / \nu\right) /(2 \pi)\right]^{n}$ in $\mathcal{I}^{[k]}(\nu)$, Eq. (230). We have seen analytically in Secs. XXI and XXII and in Appendix A that $\mathcal{I}_{n}^{[k]}(\nu)$ for $k \geq 2$ contains no more than $n-1$ powers of $\log (\nu)$ for large $\nu$.

The first nontrivial example of this is that $\mathcal{I}_{2}^{[2]}(\nu)$, when calculated at large $\log (\nu)$, is proportional to $\log (\nu)$ plus a constant but has no $\log ^{2}(\nu)$ contribution. Similarly, $\mathcal{I}_{3}^{[2]}(\nu)$ has at most a $\log ^{2}(\nu)$ contribution at large $\nu$. We can check these results numerically.

We define the second order term in the exponent in $\tilde{g}(\nu)$, Eq. (231):

$$
\left\langle\mathcal{I}^{[2]}(\nu)\right\rangle=\int_{0}^{Q^{2}} \frac{d \mu^{2}}{\mu^{2}}\left(1\left|\mathcal{S}_{\mathcal{Y}}^{[2]}\left(\mu^{2} ; \nu\right)\right|\{p, f, c, c\}_{2}\right) .
$$

We expand $\left\langle\mathcal{I}^{[2]}(\nu)\right\rangle$ in powers of $\alpha_{s}\left(Q^{2} / \nu\right) /(2 \pi)$ and calculate numerically the first two coefficients, $\left\langle\mathcal{I}_{2}^{[2]}(\nu)\right\rangle$ and $\left\langle\mathcal{I}_{3}^{[2]}(\nu)\right\rangle$,

$$
\begin{aligned}
\left\langle\mathcal{I}^{[2]}(\nu)\right\rangle= & \left\langle\mathcal{I}_{2}^{[2]}(\nu)\right\rangle\left(\frac{\alpha_{s}\left(Q^{2} / \nu\right)}{2 \pi}\right)^{2} \\
& +\left\langle\mathcal{I}_{3}^{[2]}(\nu)\right\rangle\left(\frac{\alpha_{s}\left(Q^{2} / \nu\right)}{2 \pi}\right)^{3}+\cdots
\end{aligned}
$$

The state $\left.\mid\{p, f, c, c\}_{2}\right)$ in Eq. (248) is a color singlet, flavor singlet, $q \bar{q}$ state with $p_{1}+p_{2}=Q$. The results are the same with any quark flavor choice and there is only one possible color state. The state is normalized to $\left(1 \mid\{p, f, c, c\}_{2}\right)=1$. The operator $\mathcal{S}_{\mathcal{Y}}^{[2]}\left(\mu^{2} ; \nu\right)$ is calculated using the exact DEDUCTOR splitting functions according to Eq. (175). We use the exact definition of thrust to calculate $\tau$ in $\mathcal{O}(\nu)$, Eq. (137). The calculation is performed with full color, not just leading color or the LC+ approximation. The integrals over scale in $\left\langle\mathcal{I}_{n}^{[2]}(\nu)\right\rangle$ are infrared convergent so there is no need to impose a lower cutoff on the shower scale $\mu^{2}$. Then the coefficients $\left\langle\mathcal{I}_{n}^{[2]}(\nu)\right\rangle$ are independent of $Q^{2}$.

We plot $\left\langle\mathcal{I}_{2}^{[2]}(\nu)\right\rangle$ versus $\log (\nu)$ as the solid red curve in Fig. 1. We first note that $\left\langle\mathcal{I}_{2}^{[2]}(\nu)\right\rangle$ is small. For instance, $\log (\nu)=8$ corresponds roughly to $\tau=e^{-8} \approx 3 \times 10^{-4}$ in the thrust distribution. For $\log (\nu)<8$, we find $\left|\left\langle\mathcal{I}_{2}^{[2]}(\nu)\right\rangle\right| \lesssim 1$. Then if we take $\alpha_{s} \approx 0.1$, we have $\left[\alpha_{s} /(2 \pi)\right]^{2}\left|\left\langle\mathcal{I}_{2}^{[2]}(\nu)\right\rangle\right| \lesssim 0.0003$. The function $\mathcal{I}^{[2]}(\nu)$ appears in the exponent of the Laplace transform of the thrust distribution, but for such a small value of $\mathcal{I}^{[2]}(\nu)$, one would not have needed to exponentiate it.
$\Lambda$ ordering, DEDUCtor

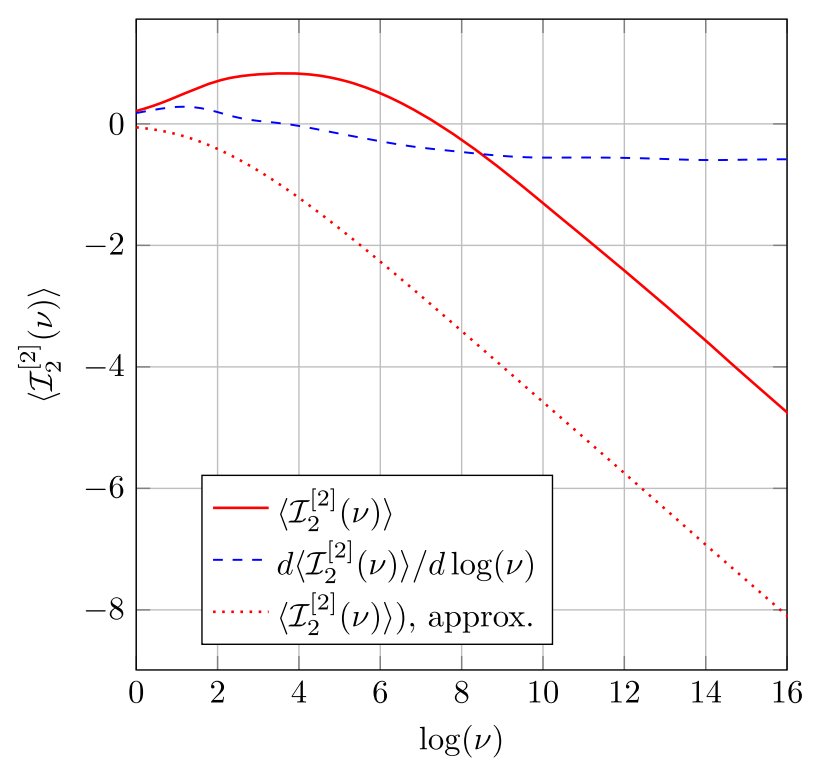

FIG. 1. Plot of $\left\langle\mathcal{I}_{2}^{[2]}(\nu)\right\rangle$, Eqs. (248) and (249), versus $\log (\nu)$ (solid red curve). For large $\log (\nu)$ the graph is approximately a straight line, corresponding to only one factor of $\log (\nu)$, indicating that the shower generates $\left\langle\mathcal{I}_{2}^{[2]}(\nu)\right\rangle$ at NLL accuracy. The dashed blue curve is $d\left\langle\mathcal{I}_{2}^{[2]}(\nu)\right\rangle / d \log (\nu)$. The dotted red curve shows an approximate version of $\left\langle\mathcal{I}_{2}^{[2]}(\nu)\right\rangle$ described in the text. These calculations and calculations of $\left\langle\mathcal{I}_{k}^{[2]}(\nu)\right\rangle$ in later figures use full QCD color.

Our primary concern is the behavior of $\left\langle\mathcal{I}_{2}^{[2]}(\nu)\right\rangle$ for very large $\log (\nu) .{ }^{10}$ Our analytical results indicate that $\left\langle\mathcal{I}_{2}^{[2]}(\nu)\right\rangle$ should be a straight line for $\operatorname{large} \log (\nu)$. The numerical result supports this conclusion. We also evaluate the integrand for $d\left\langle\mathcal{I}_{2}^{[2]}(\nu)\right\rangle / d \log (\nu)$ analytically and then integrate this expression numerically and display the result as the dashed blue curve in Fig. 1. The analytical result implies that $d\left\langle\mathcal{I}_{2}^{[2]}(\nu)\right\rangle / d \log (\nu)$ should approach a constant for large $\log (\nu)$ and the numerical result supports this conclusion.

In our analysis, we argued that $\hat{\tau}-\tau=y$ should be a good approximation in the second splitting for the purpose of determining how many powers of $\log (\nu)$ can appear in $\left\langle\mathcal{I}_{2}^{[2]}(\nu)\right\rangle$. We tried calculating $\left\langle\mathcal{I}_{2}^{[2]}(\nu)\right\rangle$ with this approximation. The result is shown as the dotted red line in Fig. 1. This curve is, as expected, a straight line for large $\log (\nu)$ and has the same slope as the curve for the exact $\left\langle\mathcal{I}_{2}^{[2]}(\nu)\right\rangle$.

\footnotetext{
${ }^{10}$ The function $\tau\left(\{p\}_{m}\right)$ is a complicated function of the parton momenta. Evaluation of this function becomes numerically unstable for parton states $\{p\}_{m}$ that give very small $\tau$. For this reason, in this and later figures, we $\operatorname{limit} \log (\nu)$ to $\log (\nu)<16$, although in some cases the numerical results appear to be reliable for larger values of $\log (\nu)$.
} 
$\Lambda$ ordering, DeDUCTOR

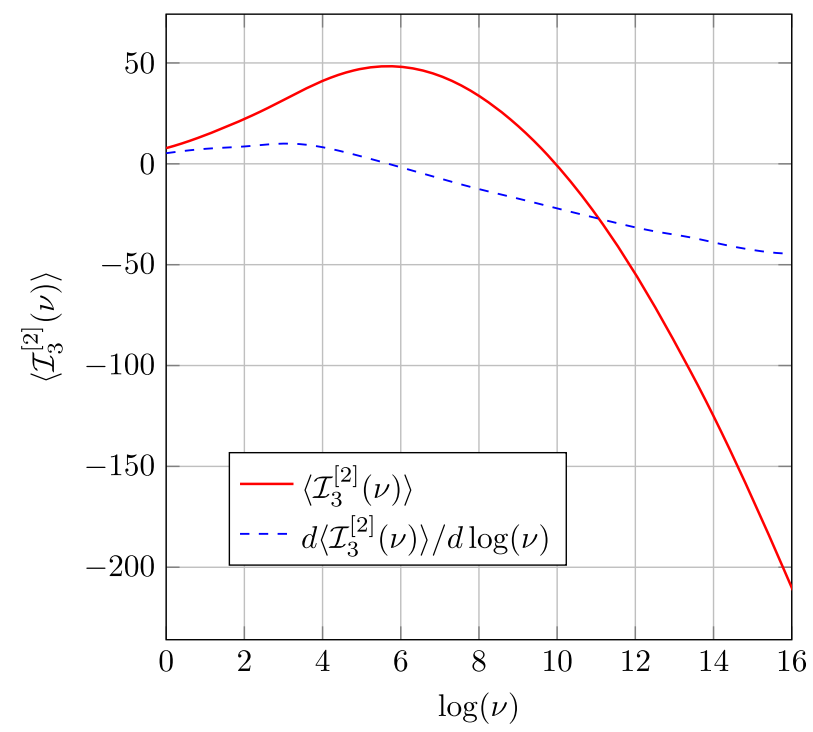

FIG. 2. Plot of $\left\langle\mathcal{I}_{3}^{[2]}(\nu)\right\rangle$, Eqs. (248) and (249), versus $\log (\nu)$ (solid red curve). The dashed blue curve is $d\left\langle\mathcal{I}_{3}^{[2]}(\nu)\right\rangle / d \log (\nu)$. For large $\log (\nu)$ the graph of $d\left\langle\mathcal{I}_{3}^{[2]}(\nu)\right\rangle / d \log (\nu)$ is approximately a straight line, indicating that the shower generates $\left\langle\mathcal{I}_{3}^{[2]}(\nu)\right\rangle$ at NLL accuracy.

We were a bit surprised to find that $\left\langle\mathcal{I}_{2}^{[2]}(\nu)\right\rangle$ with the exact $\hat{\tau}-\tau$ differs by a noticeable amount from the result with the approximate thrust value. The difference is in the direction of making $\left|\left\langle\mathcal{I}_{2}^{[2]}(\nu)\right\rangle\right|$ smaller. We do not have an analytical explanation for this behavior.

We also calculated $\left\langle\mathcal{I}_{3}^{[2]}(\nu)\right\rangle$ as a numerical integral. We plot $\left\langle\mathcal{I}_{3}^{[2]}(\nu)\right\rangle$ versus $\log (\nu)$ as the solid red curve in Fig. 2. We note first that $\left[\alpha_{s} /(2 \pi)\right]^{3}\left|\left\langle\mathcal{I}_{3}^{[2]}(\nu)\right\rangle\right|$ is small for $\log (\nu)<8$ if we take $\alpha_{s} \approx 0.1$. Our analytical results indicate that for large $\nu$ the highest power of $\log (\nu)$ in $\left\langle\mathcal{I}_{3}^{[2]}(\nu)\right\rangle$ should be $\log ^{2}(\nu)$. This implies that for large $\nu$ the highest power of $\log (\nu)$ in $d\left\langle\mathcal{I}_{3}^{[2]}(\nu)\right\rangle / d \log (\nu)$ should be $\log ^{1}(\nu)$. The numerical result, graphed as the dashed blue line in Fig. 2, supports this conclusion.

\section{NUMERICAL BEHAVIOR OF THE THRUST DISTRIBUTION}

We have seen that the operator $\mathcal{S}_{\mathcal{Y}}\left(\mu^{2} ; \nu\right)$ directly generates the Laplace transform $\tilde{g}(\nu)$ of the thrust distribution according to Eq. (231). The first order term $\mathcal{S}_{\mathcal{Y}}^{[1]}\left(\mu^{2} ; \nu\right)$ in this operator is obtained from the shower splitting function for a first order $\Lambda$-ordered parton shower. We have further seen that this term generates the known [35] summation of logarithms of $\tau$ at the NLL level as long as the shower splitting function is suitably defined. Furthermore, the higher order terms $\mathcal{S}_{\mathcal{Y}}^{[k]}\left(\mu^{2} ; \nu\right)$ obtained from this first order shower splitting function generate only contributions beyond the NLL level.

According to Eq. (227), the same result for $\tilde{g}(\nu)$ as in Eq. (231) is obtained by running the $\Lambda$-ordered shower and measuring the Laplace transform of the thrust distribution. However, we do not need to take the Laplace transform. We can simply run the $\Lambda$-ordered shower and measure the thrust distribution $g(\tau)$, as in Eq. (134). Will this give the same result as the NLL analytical result listed in Eqs. (221) and (222)?

In this section, we try this experiment. It is not useful to set $Q^{2}=M_{\mathrm{Z}}^{2}$, which would be relevant for LEP (large electron positron) experiments because a parton shower needs an infrared cutoff. We can take the cutoffs on allowed shower splittings to be $\Lambda>1 \mathrm{GeV}$ and $k_{\mathrm{T}}>1 \mathrm{GeV}$, but then there is not much range between $(1 \mathrm{GeV})^{2}$ and the starting scale $Q^{2}$ of the shower. The result is that there is not a wide range in $\tau$ in which we can examine the dependence of $g(\tau)$ on $\log (1 / \tau)$ free of the effects of the infrared cutoffs. Instead, we retain $(1 \mathrm{GeV})^{2}$ cutoffs but set $Q^{2}=(10 \mathrm{TeV})^{2}$. We then run the $\Lambda$-ordered Deductor shower with the LC+ approximation for color [22]. We turn off the top quark, so that the shower is based on 5-flavor QCD.

We compare $\tau g(\tau)$ according to DedUCTOR with $\tau g(\tau)$ according to the NLL formula, Eqs. (221) and (222), in Fig. 3. We see that the DEDUCTOR curve is a bit higher than the NLL curve around $\tau=0.01$ and a bit lower at the

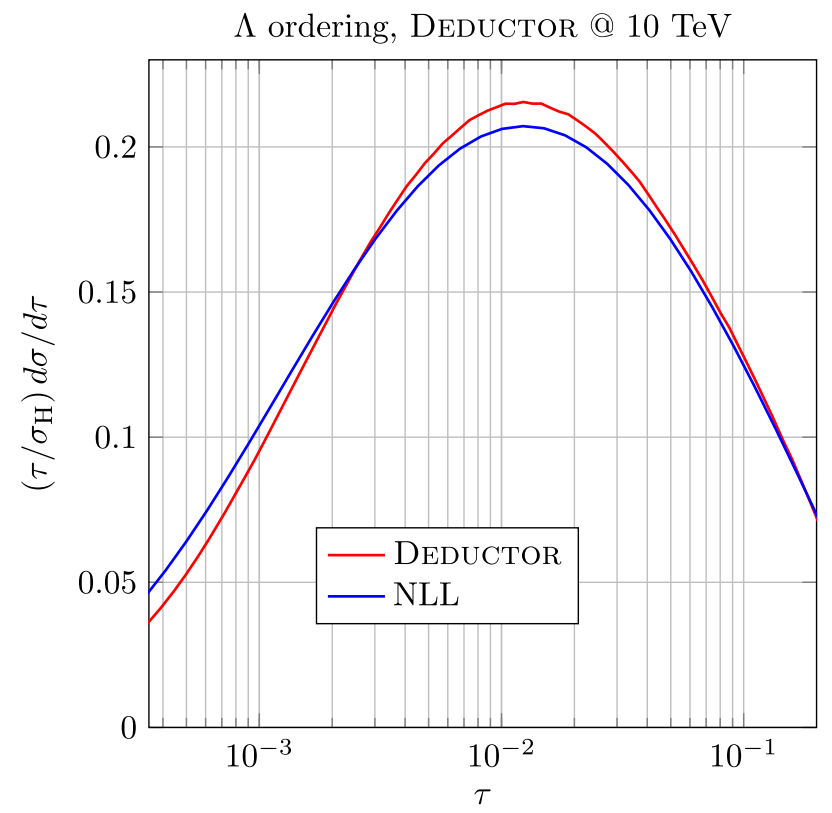

FIG. 3. Plot of $\left(\tau / \sigma_{\mathrm{H}}\right) d \sigma / d \tau$ according to DeDUCTOR with $\Lambda$ ordering at $Q^{2}=(10 \mathrm{TeV})^{2}$ compared to the NLL expectation, Eqs. (221) and (222). In DEDUCTOR, we use a cutoff for splittings: $k_{\mathrm{T}}>1 \mathrm{GeV}$ and $\Lambda>1 \mathrm{GeV}$. The Deductor curve is higher than the NLL curve at $\tau \approx 0.01$ and lower for small $\tau$. The DEDUCTOR calculation uses the LC+ approximation for color. 
smallest values of $\tau$. Generally, the results agree to within about 0.01 .

Do these results agree within the expected errors?

(i) The DEDUCTOR shower produces contributions beyond the NLL level. If we look at $\tau=0.01$ so that $\log (1 / \tau)=4.6$, NNLL terms lack a factor 4.6 compared to NLL terms. A simple calculation shows that the NLL terms contribute approximately -0.03 to $\tau g(\tau)$ at $\tau=0.01$. Thus we might expect that the NNLL terms in DEDUCTOR would contribute $\pm 0.03 / 4.6 \approx \pm 0.007$ to $\tau g(\tau)$. This gives us an error estimate from terms in DEDUCTOR beyond NLL of \pm 0.007 .

(ii) There are typically about 20 parton splittings between the $10 \mathrm{TeV}$ scale at which the shower starts and the $1 \mathrm{GeV}$ scale at which it ends. We cannot be confident that there are not $0.1 \%$ errors for each splitting resulting from approximations within the DEDUCTOR code, so we cannot rule out a $2 \%$ systematic error in $g(\tau)$ resulting from these approximations. A $2 \%$ error on the value $\tau g(\tau) \approx 0.2$ at $\tau=0.01$ amounts to an error of \pm 0.004 in $\tau g(\tau)$.

(iii) The infrared cutoffs have some effect. The most important effect comes from the limit on the transverse momentum in a splitting, which we set to $k_{\mathrm{T}}>1 \mathrm{GeV}$. To test for sensitivity to this cutoff, we change the cut to $k_{\mathrm{T}}>3 \mathrm{GeV}$. In the range $0.0005<\tau<0.2$, we find that this change in cutoff produces a change in $\tau g(\tau)$ that is generally smaller than 0.003 . Thus we estimate an error of \pm 0.003 in $\tau g(\tau)$ due to the influence of the infrared cutoff.

(iv) The DeDuctor splitting kernel omits the $\beta_{1}$ term in Eq. (216) for evaluating the dependence of $\alpha_{s}((1-$ $\left.z) y Q^{2}\right)$ on $(1-z)$. This changes the DeDUCTOR result at the NLL level. We examine this effect below.

(v) The LC+ approximation used by default in DeDuCTOR is not the same as exact color. This can introduce spurious terms of order $1 / N_{c}^{2}$ times logarithms of $1 / \tau$ into the LC+ DEDUCTOR result, where $N_{c}=3$ is the number of colors. We examine this effect below.

We examine the effects of missing NLL terms and of color in Fig. 4. Here the NLL curve is copied from Fig. 3 and the DEDUCtor curve from Fig. 3 is displayed as a dashed (black) line. The remaining two curves are modified versions of the curves in Fig. 3.

We first address the fact that Deductor omits the $\beta_{1}$ term for evaluating the dependence of $\alpha_{s}\left((1-z) y Q^{2}\right)$ on $(1-z)$. This means that in the summation of logarithms of $\log (1 / \tau)$, DEDUCTOR is missing the term $-\left(\beta_{1} / \beta_{0}\right) \log \left(\left(1-\lambda^{2}\right) /(1-2 \lambda)\right)$ in $f_{2}(\lambda)$ in Eq. (225). In order to see the effect of this term, we calculate the ratio

$$
r(\tau)=\frac{g_{\mathrm{NLL}}(\tau)}{g_{\mathrm{NLL}}^{\bmod }(\tau)}
$$

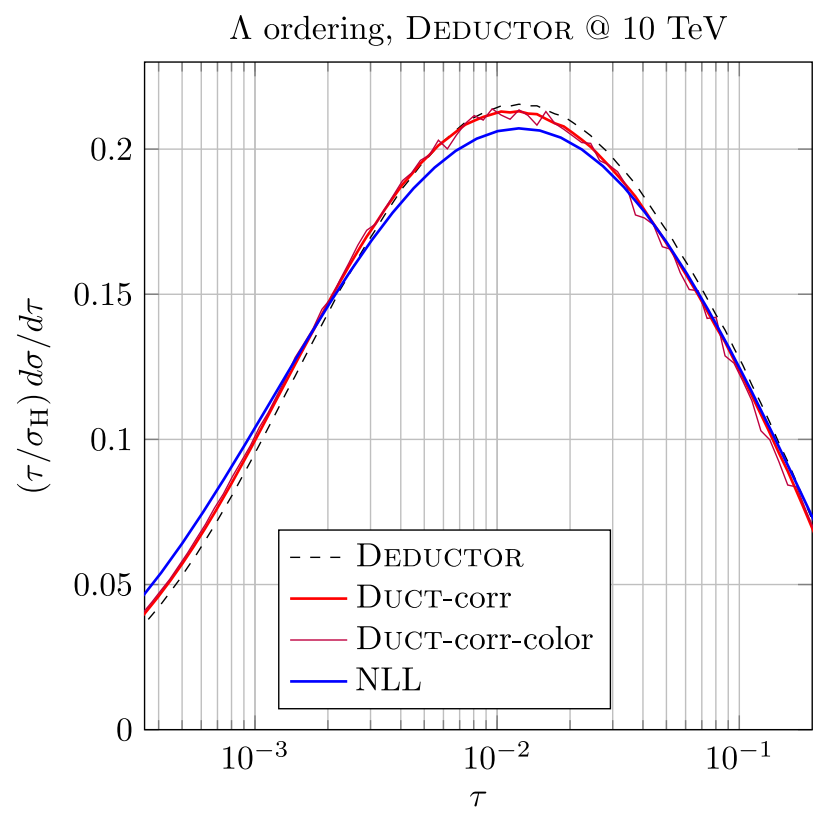

FIG. 4. Plots of $\left(\tau / \sigma_{\mathrm{H}}\right) d \sigma / d \tau$ using DEDUCTOR with $\Lambda$ ordering at $Q^{2}=(10 \mathrm{TeV})^{2}$. The black dashed curve is the DeDuCtor curve from Fig. 3. The blue solid curve is the NLL formula from Fig. 3. The red solid curve is the DEDUCTOR result corrected with the factor $r(\tau)$ to include more exact $\alpha_{\mathrm{s}}$ evolution. The purple solid curve, with noticeable statistical fluctuations, is the corrected DEDUCTOR result with two units of extra color beyond the $\mathrm{LC}+$ approximation.

where $g_{\mathrm{NLL}}^{\bmod }(\tau)$ is obtained by omitting the term $-\left(\beta_{1} / \beta_{0}\right) \log \left(\left(1-\lambda^{2}\right) /(1-2 \lambda)\right)$ in the calculation of $g(\tau)$. Then we correct the DEDUCTOR result for $g(\tau)$ by multiplying it by $r(\tau)$. We plot the corrected DEDUCTOR curve in Fig. 4. We see that the corrected DEDUCTOR curve is quite close to the uncorrected curve. However the difference is visible in Fig. 4 and acts in the direction of reducing the discrepancy between the analytical summation of logarithms and the numerical DEDUCTOR result. ${ }^{11}$

We next address the fact that in Fig. 3 we used the default color approximation in DEDUCTOR, the LC+ approximation [22]. This approximation is an improvement over the leading color approximation, but it is far from being exact. In the LC+ approximation, we replace the exact first order splitting function $\mathcal{S}\left(\mu^{2}\right)=\mathcal{S}^{[1,0]}\left(\mu^{2}\right)+\mathcal{S}^{[0,1]}\left(\mu^{2}\right)$ by an approximate version $\mathcal{S}_{\mathrm{LC}+}\left(\mu^{2}\right)=\mathcal{S}_{\mathrm{LC}+}^{[1,0]}\left(\mu^{2}\right)+\mathcal{S}_{\mathrm{LC}+}^{[0,1]}\left(\mu^{2}\right)$. DEDUCTOR has the option of expanding in powers of $\mathcal{S}\left(\mu^{2}\right)-\mathcal{S}_{\mathrm{LC}+}\left(\mu^{2}\right)$ and keeping terms up to and including $\left[\mathcal{S}\left(\mu^{2}\right)-\mathcal{S}_{\mathrm{LC}+}\left(\mu^{2}\right)\right]^{n}$, where $n$ can be chosen by the user [31]. In order to assess what difference a more exact treatment of color could make, we plot in Fig. 4 the result of calculating the thrust distribution at $10 \mathrm{TeV}$ with $n=2$ for

\footnotetext{
${ }^{11}$ In a future version of DEDUCTOR, we may add this contribution to the splitting kernel, although its practical effect is quite small.
} 
those splittings that have $\Lambda>10 \mathrm{GeV}$. We have corrected this result using the factor $r(\tau)$ from Eq. (250). Of course, using $n=2$ slows the calculation down, increasing the statistical errors. Within the statistical errors, we find that improving the color treatment makes no difference.

In summary, we have made a numerical comparison of the expected NLL result for the thrust distribution and a direct calculation using a $\Lambda$-ordered parton shower with a global momentum mapping, setting $Q^{2}$ to $(10 \mathrm{TeV})^{2}$ so as to allow $\log (1 / \tau)$ to be adequately large to provide a real test. We have found good agreement within the estimated errors.

\section{COLOR AND LOGARITHMS}

Our analysis in this paper mostly does not examine the effect of approximating color on the summation of logarithms in the thrust distribution. For instance, the analytical analysis in Secs. XXI and XXII and the numerical results in Figs. 1 and 2 use exact QCD color. The DEDUCTOR result in Fig. 3 is calculated with the LC+ approximation, which is a numerically very good color approximation for the thrust distribution, as seen in Fig. 4. It would certainly be of interest to examine analytically or numerically as in Figs. 1 and 2 whether the use of the LC+ approximation preserves the NLL accuracy of a parton shower that has NLL accuracy with exact color. Such a study is beyond the scope of this paper. However, we provide some brief comments on color approximations in this section.

Typically, parton shower event generators use the LC approximation $[4,27,28]$. In the simplest formulation, the LC approximation is obtained by using $\mathrm{U}(3)$ as the color group and then dropping all terms suppressed by a factor $1 / N_{c}^{2}$. Then at every step of the shower the color state $\left\{c, c^{\prime}\right\}_{m}$ has $\left\{c^{\prime}\right\}_{m}=\{c\}_{m}$ and there is a factor $C_{\mathrm{A}} / 2$ for every gluon emission vertex. At some places, one can include a $1 / N_{c}^{2}$ correction by changing $C_{\mathrm{A}} / 2$ to $C_{\mathrm{F}}=\left(C_{\mathrm{A}} / 2\right)\left(1-1 / N_{c}^{2}\right)$.

DEDUCTOR uses the LC+ approximation [22]. The generator $\mathcal{S}_{\mathrm{LC}+}\left(\mu^{2}\right)$ of splittings with the $\mathrm{LC}+$ approximation differs from the generator $\mathcal{S}\left(\mu^{2}\right)$ with full color. ${ }^{12}$ Define

$$
\Delta \mathcal{S}\left(\mu^{2}\right)=\mathcal{S}\left(\mu^{2}\right)-\mathcal{S}_{\mathrm{LC}+}\left(\mu^{2}\right) .
$$

For the first splitting from the initial $q \bar{q}$ state in $e^{+} e^{-}$ annihilation, the LC+ approximation is exact in color

$$
\left.\Delta \mathcal{S}\left(\mu^{2}\right) \mid\{p, f, c, c\}_{2}\right)=0 .
$$

Furthermore, as noted in Sec. XI, for later splittings the difference $\Delta \mathcal{S}\left(\mu^{2}\right)$ is singular only for fixed angle soft

\footnotetext{
${ }^{12}$ The LC+ approximation, including the calculation of the overlap $\left\langle\left\{c^{\prime}\right\}_{m} \mid\{c\}_{m}\right\rangle$ at the end of each event, is computationally efficient. The code for $\left\langle\left\{c^{\prime}\right\}_{m} \mid\{c\}_{m}\right\rangle$ is available in the DEDUCTOR code at [37] and [38].
}

splittings but not for collinear splittings or soft $\times$ collinear splittings [22].

We can illustrate this with an example adapted from a 1993 paper [39] by Gustafson that sorted out the $C_{\mathrm{A}} / 2$ versus $C_{\mathrm{F}}$ choice for $q \mathrm{gg} \bar{q}$ production in electron-positron annihilation. Let us start with a state containing a quark with momentum $p_{1}$, an antiquark with momentum $p_{2}$ and a gluon with momentum $p_{3}$. Now we can emit the fourth, soft, gluon with momentum $\hat{p}_{4}$. The other partons have momenta $\hat{p}_{1}, \hat{p}_{2}, \hat{p}_{3}$ after the splitting. We take $\hat{p}_{4}$ to be very small, so that we can neglect recoil and take $\hat{p}_{i}=p_{i}$ for $i \in\{1,2,3\}$. We denote the energy of $\hat{p}_{i}$ in the rest frame of the total momentum $Q$ by $E_{i}$ and we denote the angles between $\hat{p}_{i}$ and $\hat{p}_{j}$ in the rest frame of $Q$ by $\theta_{i j}$. We assume that $\theta_{12}$ is not small since $\theta_{12} \approx \pi$ most probably and $\theta_{12} \ll 1$ is not probable. Without loss of generality, we can assume that $\theta_{13}<\theta_{23}$. We consider both the possibility that $\theta_{13}$ is of order 1 and the possibility that $\theta_{13} \ll 1$.

For the emission of gluon 4 use just the dipole approximation for soft gluon emissions. With this approximation, the emission probability is

$$
\Phi_{l k}=A_{l k}^{\prime} \bar{w}_{l k}^{\text {dipole }} .
$$

Here $\bar{w}_{l k}^{\text {dipole }}$ is the familiar probability density for emitting a soft gluon with index 4 from parton $l$ with interference from emitting the same soft gluon from a different parton $k$,

$$
\bar{w}_{l k}^{\text {dipole }}=4 \pi \alpha_{s} \frac{2 \hat{p}_{k} \cdot \hat{p}_{l}}{\hat{p}_{4} \cdot \hat{p}_{k} \hat{p}_{4} \cdot \hat{p}_{l}} .
$$

This probability density is symmetric under interchange of $l$ with $k$. In a partitioned dipole shower like DEDUCTOR, as distinct from an antenna dipole shower line VINCIA [40], we distinguish the emitting parton $l$ from the dipole partner parton $k$ by multiplying by a partitioning function $A_{l k}^{\prime}$ with $A_{l k}^{\prime}+A_{k l}^{\prime}=1$. Deductor uses

$$
A_{l k}^{\prime}\left(\{\hat{p}\}_{m+1}\right)=\frac{\hat{p}_{4} \cdot \hat{p}_{k} \hat{p}_{l} \cdot Q}{\hat{p}_{4} \cdot \hat{p}_{k} \hat{p}_{l} \cdot Q+\hat{p}_{4} \cdot \hat{p}_{l} \hat{p}_{k} \cdot Q},
$$

where $Q$ is the total momentum of the final state.

The emission probability is accompanied by an operator on the partonic color state. Let us call the color state after the emission

$$
C_{l k}=\sum_{\left\{c, c^{\prime}\right\}_{4}}\left|\{c\}_{4}\right\rangle g_{l k}\left(\left\{c, c^{\prime}\right\}_{4}\right)\left\langle\left\{c^{\prime}\right\}_{4}\right| .
$$

Here $\left|\{c\}_{4}\right\rangle$ are color basis states for $q \mathrm{gg} \bar{q}$ states in the "trace" or "string" basis used in DeDUCTOR [19]. In the notation of Ref. [19], we will need basis states $\left[1, i_{3}, i_{4}, 2\right]$ for a state with a quark with index 1 and an antiquark with index 2 joined by a color string with gluons $i_{3}, i_{4}$ along the string. The probability associated with this color state is then 


$$
\operatorname{Tr} C_{l k}=\sum_{\left\{c, c^{\prime}\right\}_{4}}\left\langle\left\{c^{\prime}\right\}_{4} \mid\{c\}_{4}\right\rangle g_{l k}\left(\left\{c, c^{\prime}\right\}_{4}\right)
$$

The color state of the starting $q \mathrm{~g} \bar{q}$ state, in the notation used for $q \operatorname{gg} \bar{q}$ states, is

$$
\begin{aligned}
& \left|\{c\}_{3}\right\rangle=|[1,3,2]\rangle, \\
& \left\langle\left\{c^{\prime}\right\}_{3}\right|=\langle[1,3,2]| .
\end{aligned}
$$

After the emission of the soft gluon, the statistical state is proportional to

$$
\begin{aligned}
\frac{2}{C_{\mathrm{F}}} C_{31}= & (|[1,4,3,2]\rangle-|[1,3,4,2]\rangle)\langle[1,4,3,2]| \\
& +|[1,4,3,2]\rangle(\langle[1,4,3,2]|-\langle[1,3,4,2]|), \\
\frac{2}{C_{\mathrm{F}}} C_{32}= & (|[1,3,4,2]\rangle-|[1,4,3,2]\rangle)\langle[1,3,4,2]| \\
& +|[1,3,4,2]\rangle(\langle[1,3,4,2]|-\langle[1,4,3,2]|), \\
\frac{2}{C_{\mathrm{F}}} C_{13}= & (|[1,4,3,2]\rangle-|[1,3,4,2]\rangle)\langle[1,4,3,2]| \\
& +|[1,4,3,2]\rangle(\langle[1,4,3,2]|-\langle[1,3,4,2]|), \\
\frac{2}{C_{\mathrm{F}}} C_{23}= & (|[1,3,4,2]\rangle-|[1,4,3,2]\rangle)\langle[1,3,4,2]| \\
\times & |[1,3,4,2]\rangle(\langle[1,3,4,2]|-\langle[1,4,3,2]|), \\
\frac{2}{C_{\mathrm{F}}} C_{12}= & |[1,4,3,2]\rangle\langle[1,3,4,2]| \\
& +|[1,3,4,2]\rangle\langle[1,4,3,2]|, \\
\frac{2}{C_{\mathrm{F}}} C_{21}= & |[1,3,4,2]\rangle\langle[1,4,3,2]| \\
& +|[1,4,3,2]\rangle\langle[1,3,4,2]| .
\end{aligned}
$$

The trace of these states (using the normalization conventions of Ref. [19]) is

$\operatorname{Tr} C_{31}=\operatorname{Tr} C_{32}=\operatorname{Tr} C_{13}=\operatorname{Tr} C_{23}=T_{\|}-T_{\times}$,

$\operatorname{Tr} C_{12}=\operatorname{Tr} C_{21}=T_{\times}$,

where

$$
\begin{aligned}
T_{\|} & =C_{\mathrm{F}}, \\
T_{\times} & =-\frac{1}{2 N_{c}} .
\end{aligned}
$$

This is with full color. We can use the LC+ approximation, which is a very simple approximation on the color operators [22]. Let us define the difference between the color states obtained with full color and the color states obtained with the LC+ approximation:

$$
\Delta C_{l k}=C_{l k}-C_{l k}^{\mathrm{LC}+} .
$$

Then the LC+ approximation [22] gives

$$
\begin{aligned}
\Delta C_{31}= & 0, \\
\Delta C_{32}= & 0, \\
\Delta C_{13}= & -\left(C_{\mathrm{F}} / 2\right)|[1,4,3,2]\rangle\langle[1,3,4,2]| \\
& -\left(C_{\mathrm{F}} / 2\right)|[1,3,4,2]\rangle\langle[1,4,3,2]|, \\
\Delta C_{23}= & -\left(C_{\mathrm{F}} / 2\right)|[1,3,4,2]\rangle\langle[1,4,3,2]| \\
& -\left(C_{\mathrm{F}} / 2\right)|[1,4,3,2]\rangle\langle[1,3,4,2]|, \\
\Delta C_{12}= & \left(C_{\mathrm{F}} / 2\right)|[1,4,3,2]\rangle\langle[1,3,4,2]| \\
& +\left(C_{\mathrm{F}} / 2\right)|[1,3,4,2]\rangle\langle[1,4,3,2]|, \\
\Delta C_{21}= & \left(C_{\mathrm{F}} / 2\right)|[1,3,4,2]\rangle\langle[1,4,3,2]| \\
& +\left(C_{\mathrm{F}} / 2\right)|[1,4,3,2]\rangle\langle[1,3,4,2]| .
\end{aligned}
$$

The traces of these states are

$$
\begin{aligned}
& \operatorname{Tr} \Delta C_{31}=\operatorname{Tr} \Delta C_{32}=0, \\
& \operatorname{Tr} \Delta C_{13}=\operatorname{Tr} \Delta C_{23}=-T_{\times}, \\
& \operatorname{Tr} \Delta C_{12}=\operatorname{Tr} \Delta C_{21}=T_{\times} .
\end{aligned}
$$

This shows us what the LC+ approximation leaves out in a shower that has just two soft gluon emissions:

$$
\sum_{l} \sum_{k \neq l} \Phi_{l k} \operatorname{Tr} \Delta C_{l k}=\left(\Phi_{12}-\Phi_{13}\right) T_{\times}+\left(\Phi_{21}-\Phi_{23}\right) T_{\times} .
$$

We note first that this is color suppressed compared to the result using full color, $C_{l k}$, since a factor $1 /\left(2 N_{c}\right)$ replaces a factor $C_{\mathrm{F}}$. Second, we see immediately from Eqs. (253), (254), and (255) that $\left(\Phi_{12}-\Phi_{13}\right)$ is not singular when $\hat{p}_{4}$ becomes collinear with $\hat{p}_{1}, \hat{p}_{3}$, or $\hat{p}_{2}$. Similarly $\left(\Phi_{21}-\right.$ $\left.\Phi_{23}\right)$ has no collinear singularities. These functions still have a soft singularity: they have a $1 / E_{4}^{2}$ singularity when $E_{4} \rightarrow 0$ at a fixed $\theta_{14}, \theta_{24}$, and $\theta_{34}$.

Although $E_{4}^{2} \sum \Phi_{l k} \operatorname{Tr} \Delta C_{l k}$ is never singular, it can be large in certain angular regions. Consider the case that $\theta_{13} \ll 1$. Then $E_{4}^{2}\left(\Phi_{21}-\Phi_{23}\right)$ is never large. Additionally, $E_{4}^{2}\left(\Phi_{12}-\Phi_{13}\right)$ is not large when $\theta_{14} \ll \theta_{13}$ because $\Phi_{13}$ cancels $\Phi_{12}$ in this limit. However, when $\theta_{13} \ll \theta_{14}, E_{4}^{2} \Phi_{13}$ is small and $E_{4}^{2} \Phi_{12} \propto 1 / \theta_{14}^{2}$ is large when $\theta_{13} \ll \theta_{14} \ll 1$. Thus we can approximate

$$
\sum_{l} \sum_{k \neq l} \Phi_{l k} \operatorname{Tr} \Delta C_{l k} \approx \theta\left(\theta_{13}<\theta_{14}\right) \Phi_{12} T_{\times}
$$


This is a good approximation when $\theta_{13} \ll 1$ and works also when $\theta_{13}$ is not small since then $\theta\left(\theta_{13}<\theta_{14}\right) E_{4}^{2} \Phi_{12}$ is never large.

We have seen that the LC+ approximation is sufficient to capture most of the enhanced contributions to $E_{4}^{2} \sum \Phi_{l k} \operatorname{Tr} C_{l k}$, leaving just the contribution in Eq. (266) (assuming $\theta_{13}<\theta_{23}$ ). Using the LC+ approximation, the probability density associated with the emission of gluon 4 is

$$
\sum_{l} \sum_{k \neq l} \Phi_{l k} \operatorname{Tr} C_{l k}^{\mathrm{LC}+}=\left(\Phi_{31}+\Phi_{32}\right) \frac{C_{\mathrm{A}}}{2}+\left(\Phi_{13}+\Phi_{23}\right) C_{\mathrm{F}} .
$$

Compare the terms proportional to $\Phi_{31}$ and $\Phi_{13}$. The emission probabilities $\Phi_{31}$ and $\Phi_{13}$ contain the same dipole factor $\bar{w}_{31}^{\text {dipole }}=\bar{w}_{13}^{\text {dipole }}$. They differ in their partitioning factors $A_{31}^{\prime}$ and $A_{13}^{\prime}$, which are positive and satisfy $A_{31}^{\prime}+A_{13}^{\prime}=1$. The factor $A_{31}^{\prime}$ is dominant when the direction of the momentum $\hat{p}_{4}$ of the new gluon is closer to the direction of the first gluon than it is to the direction of the quark. In this case, the color factor in the emission probability is $C_{\mathrm{A}} / 2$. The factor $A_{13}^{\prime}$ is dominant when the direction of $\hat{p}_{4}$ is closer to the direction of the quark than it is to the direction of the first gluon. In this case, the color factor in the emission probability is $C_{\mathrm{F}}$. The analogous conclusion applies to the dipole formed by the first gluon and the antiquark.

Adding the contribution from Eq. (266), we have (assuming $\theta_{13}<\theta_{23}$ )

$$
\begin{aligned}
\sum_{l} \sum_{k \neq l} \Phi_{l k} \operatorname{Tr} C_{l k} \approx & \left(\Phi_{31}+\Phi_{32}\right) \frac{C_{\mathrm{A}}}{2}+\left(\Phi_{13}+\Phi_{23}\right) C_{\mathrm{F}} \\
& -\theta\left(\theta_{13}<\theta_{14}\right) \Phi_{12} \frac{1}{2 N_{c}} .
\end{aligned}
$$

The added term is important when $\theta_{13} \ll \theta_{14} \ll 1$. In this region, $\Phi_{12} \approx \Phi_{32}$ and the added term changes the coefficient of $\Phi_{32}$ from $C_{\mathrm{A}} / 2$ to $C_{\mathrm{F}}$.

We have discussed the case of four partons in electronpositron annihilation. For cases with an arbitrary number of partons, the LC+ approximation remains accurate up to corrections that may be large in some angular regions but that lack collinear singularities. The calculation of probabilities by taking the trace of the color density matrix is simple and is built into DeDUCTOR.

We have emphasized the real emission operators in the preceding discussion. There are also virtual exchange operators that create the Sudakov factor in a probability preserving shower. With full color, the virtual exchange operators can change the color vectors $\left.\mid\left\{c, c^{\prime}\right\}_{m}\right)$ to which they are applied. However, with the LC+ approximation, applying the color operators to a vector $\left.\mid\left\{c, c^{\prime}\right\}_{m}\right)$ returns just an eigenvalue times the vector. For gluon emission, the eigenvalue is either $C_{\mathrm{A}} / 2$ (for emission from a gluon) or $C_{\mathrm{F}}$ (for emission from a quark). Thus the Sudakov operators are simple in the $\mathrm{LC}+$ approximation. They are part of DEDUCTOR.

Unfortunately, we do not currently know of a way to enhance the LC+ approximation so as to incorporate contributions like those in Eq. (266) while still avoiding the possibility that the revised virtual exchange operators change the color vectors $\left.\mid\left\{c, c^{\prime}\right\}_{m}\right)$ to which they are applied, making it difficult to build the Sudakov operators in a computationally manageable way. Although we do not know of a practical way to put the virtual exchange parts of $\Delta \mathcal{S}$ into a Sudakov exponential, it is possible to calculate the contributions from $\Delta \mathcal{S}$ perturbatively [31]. Typically, we have found that these contributions are numerically small, as in Fig. 4.

The result on the right hand side of Eq. (268) was found, with a different notation, by Gustafson in 1993 [39] as being a good approximation to the full $q \mathrm{gg} \bar{q}$ cross section. The analysis used what are now called Lund diagrams. In order to account for the $1 / N_{c}$ terms that distinguish $C_{\mathrm{F}}$ from $C_{\mathrm{A}} / 2$, Gustafson called on the idea of color coherence for wide angle soft gluon emission from partons with nearly collinear momenta. According to color coherence, we are to add amplitudes, not probabilities. In the formalism of the present paper, color coherence does not need to be invoked separately. It is built in because we add color amplitudes in Eq. (259).

After the present paper was submitted [41,42], Hamilton, Medves, Salam, Scyboz and Soyez [17] extended the analysis of Gustafson to more parton emissions in electron-positron annihilation, providing prescriptions for making the choice between color factors $C_{\mathrm{A}} / 2$ or $C_{\mathrm{F}}$ for real gluon emissions. This paper omits direct analysis of color amplitudes or the effect on color amplitudes of the virtual exchanges needed to build a Sudakov operator. ${ }^{13}$ For more than four partons, a direct, term-by-term analysis like that given above is cumbersome because there are many terms and because the color state before the soft gluon emission now has $\left\{c^{\prime}\right\}_{m} \neq\{c\}_{m}$. For this reason, we do not undertake a comparison to the results of Ref. [17] here.

We have, however, undertaken a simple calculation to check the effects on the thrust distribution of degrading the LC+ approximation to just an LC approximation. DEDUCTOR has the capability to turn off the LC+ approximation at some point in the shower by changing the color group from $\mathrm{SU}(3)$ to $\mathrm{U}(3)$. With this treatment, a gluon is the same, in color, as a quark-antiquark pair. Then splittings with color connections that produce $1 / N_{c}^{2}$ factors are

\footnotetext{
${ }^{13}$ The paper states that for four or more partons "one should worry about amplitude-level evolution [43], which is beyond the accuracy and scope of this article."
} 


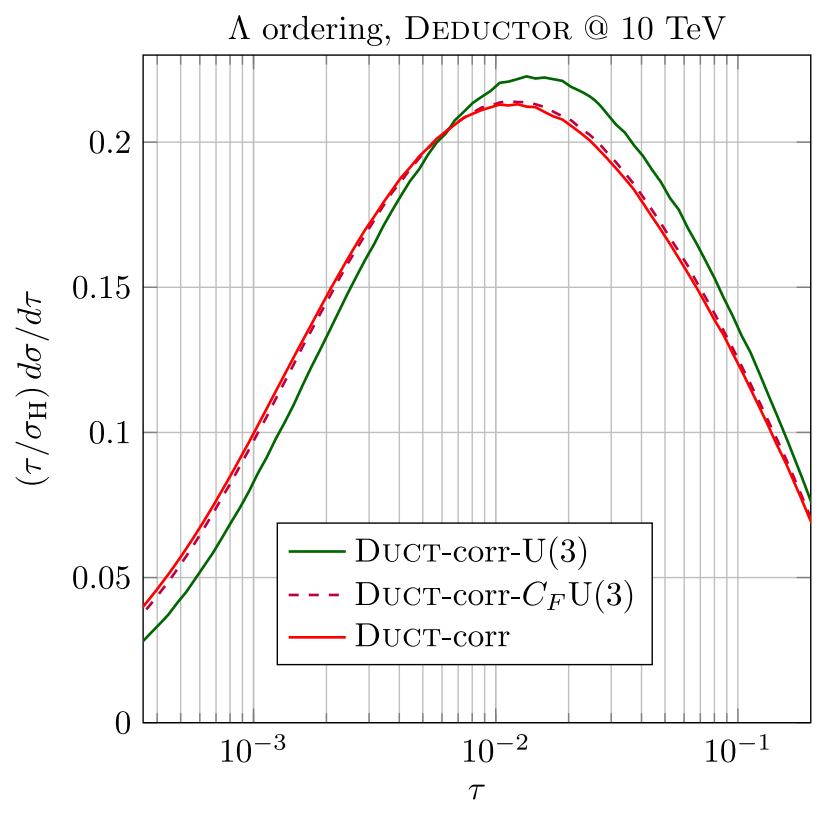

FIG. 5. Plots of $\left(\tau / \sigma_{\mathrm{H}}\right) d \sigma / d \tau$ using DEDUCTOR with $\Lambda$ ordering at $Q^{2}=(10 \mathrm{TeV})^{2}$. All three curves are DEDUCTOR results corrected with the factor $r(\tau)$ to include more exact $\alpha_{\mathrm{s}}$ evolution. The red solid curve uses the LC+ approximation and is taken from Fig. 4. In the green solid curve the color approximation is reduced from $\mathrm{LC}+$ to color using $\mathrm{U}(3)$ as the color group. This puts a factor $C_{A} / 2$ at $q q$ g vertices. The purple dashed curve also uses $\mathrm{U}(3)$ as the color group but inserts a factor $C_{\mathrm{F}}$ at the first emission.

omitted. This gives a variety of LC approximation with a factor $C_{\mathrm{A}} / 2$ factor at each splitting.

In Fig. 5, we compare the thrust distribution calculated with this $\mathrm{U}(3)$ leading color approximation to the thrust distribution calculated with the LC+ approximation, taken from Fig. 4. In both cases, we apply the correction factor $r(\tau)$ that was used in Fig. 4. We see that replacing the LC+ approximation with this LC approximation makes a substantial difference. The first splitting must be $q \rightarrow q+\mathrm{g}$, for which a factor $C_{\mathrm{F}}$ would be more sensible than a factor $C_{\mathrm{A}} / 2$, even though these are equivalent within the $\mathrm{LC}$ approximation. We tried the same calculation with $C_{\mathrm{A}} / 2$ replaced by $C_{\mathrm{F}}$ in the first splitting. This gives the dashed curve in Fig. 5. This results in substantially improving the agreement with the LC+ curve. The discrepancy is reduced by a factor of roughly 7 .

\section{XXVI. $\boldsymbol{k}_{\mathrm{T}}$ ORDERING}

The default ordering variable in DEDUCTOR is $\Lambda$, Eq. (98). However, there is an option to use $k_{\mathrm{T}}$ ordering, ${ }^{14}$ still with exact color. We can define $\mathcal{I}^{[2]}(\nu)$ with $k_{\mathrm{T}}$ ordering using Eqs. (175) and (248). We simply set the scale

\footnotetext{
${ }^{14}$ For $k_{\mathrm{T}}$ ordering, $k_{\mathrm{T}}^{2}=-k_{\perp}^{2}$ where the vector $k_{\perp}$ is orthogonal to the momentum $p_{l}$ of the emitting parton and to $Q$, rather than being orthogonal to $p_{l}$ and the momentum $p_{k}$ of the dipole partner parton.
}

parameters to $\mu^{2}=k_{T}^{2}$ for the first splitting and $\bar{\mu}^{2}=\bar{k}_{T}^{2}$ for the second splitting. Then $k_{\mathrm{T}}$ ordering means that $\bar{k}_{\mathrm{T}}^{2}<$ $k_{\mathrm{T}}^{2}$ in Eq. (175).

With $k_{\mathrm{T}}$ ordering, the reasoning supporting NLL accuracy of the $\Lambda$-ordered shower from Sec. XXI and Appendix A is lost. However, it appears that we can still get cancellation of $\log (\nu)$ factors in $\mathcal{I}_{2}^{[2]}(\nu)$ at the NLL level. That is, the integral has contributions proportional to $\log ^{4}(\nu)$ at large $\log (\nu)$, but after these contributions are summed, only terms proportional to $\log ^{1}(\nu)$ and $\log ^{0}(\nu)$ remain. The mechanism is that the contributions from the two terms specified by the $[\cdots]_{1-\mathbb{P}}$ operation in the last line of Eq. (175), representing real emissions and virtual emissions, cancel each other. A complete proof is beyond the scope of this paper, but we present an argument that makes this conclusion plausible in Appendix B.

We can check the effect of the choice of ordering variable on the summation of $\log (\nu)$ factors in the thrust distribution by calculating $\left\langle\mathcal{I}_{2}^{[2]}(\nu)\right\rangle$ numerically using the DEDUCTOR shower algorithm with $k_{\mathrm{T}}$ ordering and exact color. The result is shown as the solid red curve in Fig. 6. We see that $\left\langle\mathcal{I}_{2}^{[2]}(\nu)\right\rangle$ is quite small, $\left|\left\langle\mathcal{I}_{2}^{[2]}(\nu)\right\rangle\right|<2$ for $\log (\nu)<8$. For NLL accuracy, this curve should be linear for large $\log (\nu)$. To quite good, but not perfect, accuracy, it is.

We have also checked the behavior of $\left\langle\mathcal{I}_{3}^{[2]}(\nu)\right\rangle$ as a function of $\log (\nu)$. The results are shown in Fig. 7. For large $\nu$ the highest power of $\log (\nu)$ in $\left\langle\mathcal{I}_{3}^{[2]}(\nu)\right\rangle$ should be $\log ^{2}(\nu)$. This implies that for large $\nu$ the highest power of $\log (\nu)$ in $d\left\langle\mathcal{I}_{3}^{[2]}(\nu)\right\rangle / d \log (\nu)$ should be $\log ^{1}(\nu)$. The

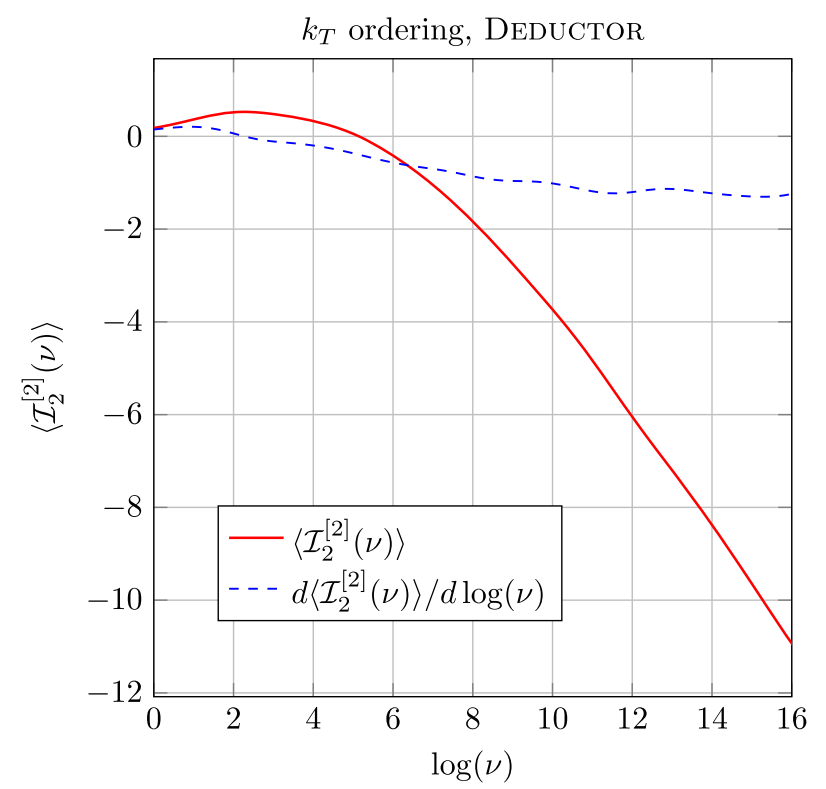

FIG. 6. Plot of $\left\langle\mathcal{I}_{2}^{[2]}(\nu)\right\rangle$ versus $\log (\nu)$, as in Fig. 1, for the DEDUCTOR shower algorithm with $k_{\mathrm{T}}$ ordering. The blue dashed curve is $d\left\langle\mathcal{I}_{2}^{[2]}(\nu)\right\rangle / d \log (\nu)$. 
$k_{T}$ ordering, DEDUCTOR

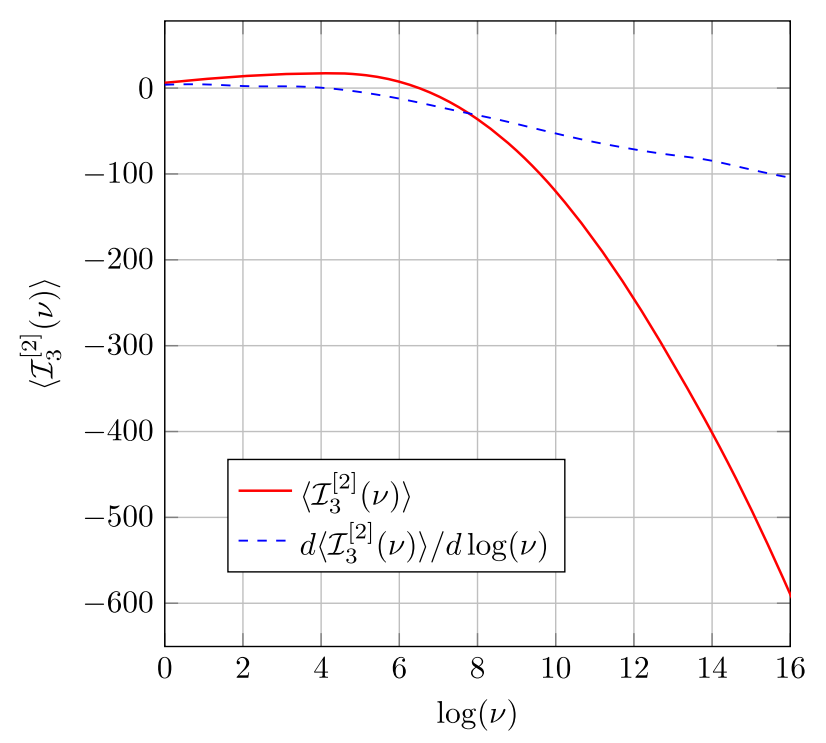

FIG. 7. Plot of $\left\langle\mathcal{I}_{3}^{[2]}(\nu)\right\rangle$ versus $\log (\nu)$, as in Fig. 2, for the DEDUCTOR shower algorithm with $k_{\mathrm{T}}$ ordering. The blue dashed curve is $d\left\langle\mathcal{I}_{3}^{[2]}(\nu)\right\rangle / d \log (\nu)$.

numerical result, graphed as the dashed blue line in Fig. 7, supports this conclusion.

We have investigated only $\left\langle\mathcal{I}_{2}^{[2]}(\nu)\right\rangle$ and $\left\langle\mathcal{I}_{3}^{[2]}(\nu)\right\rangle$. We have found results consistent with NLL accuracy for the DEDUCTOR shower with $k_{\mathrm{T}}$ ordering, but there could still be inconsistencies with NLL accuracy for $\left\langle\mathcal{I}_{n}^{[k]}(\nu)\right\rangle$ for other values of $k$ and $n$. A promising approach to investigating this issue would be to automate the calculation of $\left\langle\mathcal{I}_{n}^{[k]}(\nu)\right\rangle$ so that these functions could be calculated numerically for any nottoo-large values of $k$ and $n$. We leave this approach to future work.

We can also look directly at $\left(\tau / \sigma_{\mathrm{H}}\right) d \sigma / d \tau$ with $Q^{2}=(10 \mathrm{TeV})^{2}$. We use either DEDUCTOR with its default $\Lambda$ ordering or DEDUCTOR with $k_{\mathrm{T}}$ ordering, both with LC+ color. The result with $\Lambda$ ordering, from Fig. 4, includes the correction factor $r(\tau)$ from Eq. (250). The result with $k_{\mathrm{T}}$ ordering needs no correction factor because $k_{\mathrm{T}}^{2}$ in $\alpha_{s}\left(\lambda_{\mathrm{R}} k_{\mathrm{T}}^{2}\right)$ in the DEDUCTOR splitting function is the same as the ordering variable. We do not include hadronization. Thus we examine only perturbative effects and the effects of the shower cutoff. With $\Lambda$ ordering, the shower stops at $\Lambda=1 \mathrm{GeV}$ and there is also a cut that prevents the $k_{\mathrm{T}}$ in any splitting from being smaller than $1 \mathrm{GeV}$. With $k_{\mathrm{T}}$ ordering, the shower stops at $k_{\mathrm{T}}=1 \mathrm{GeV}$. The result is shown in Fig. 8. We see that the shower ordering does make a difference. Although $\left(\tau / \sigma_{\mathrm{H}}\right) d \sigma / d \tau$ calculated with $k_{\mathrm{T}}$ ordering is similar to the NLL expectation $\tau g(\tau)$ from Eqs. (221) and (222), the difference between these two results is greater than the expected uncertainties discussed for $\Lambda$ ordering in Sec. XXIV.
$\Lambda$ vs. $k_{\mathrm{T}}$ ordering, Deductor @ $10 \mathrm{TeV}$

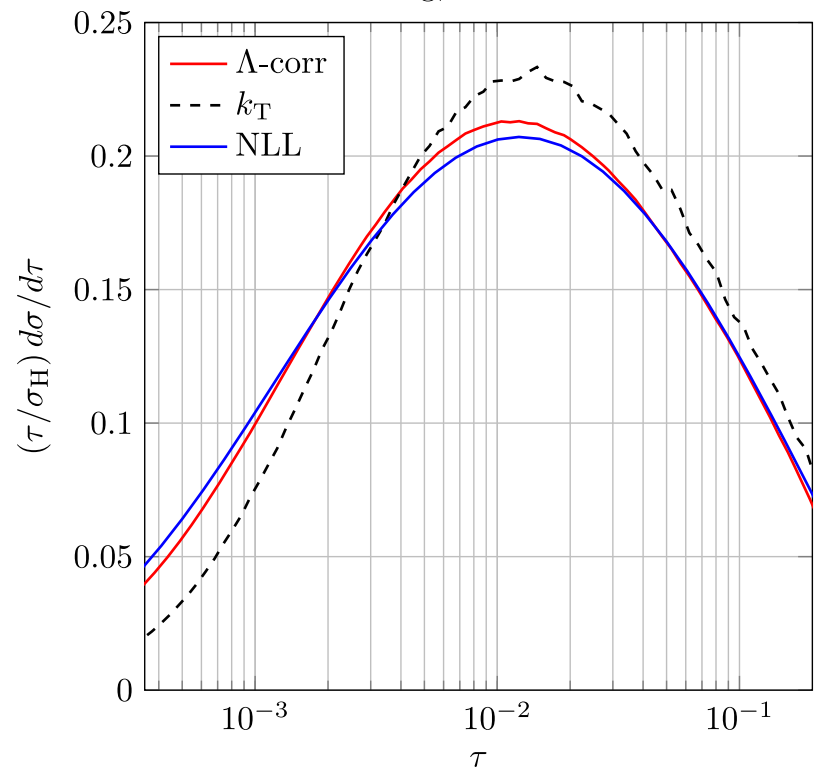

FIG. 8. Plot of $\left(\tau / \sigma_{\mathrm{H}}\right) d \sigma / d \tau$ with $\Lambda$ ordering and $k_{\mathrm{T}}$ ordering at $Q^{2}=(10 \mathrm{TeV})^{2}$. Both are compared to the NLL expectation, Eqs. (221) and (222). We use a cutoff on the transverse momentum in splittings: $k_{\mathrm{T}}>1 \mathrm{GeV}$.

As an alternative, we can follow the method of Ref. [16] and calculate $\left(\tau / \sigma_{\mathrm{H}}\right) d \sigma / d \tau$ for various values of $Q^{2}$, and thus for various values of $\alpha_{s}\left(Q^{2}\right)$. We choose $Q^{2}=$ $(1 \mathrm{TeV})^{2},(10 \mathrm{TeV})^{2}$, and $(100 \mathrm{TeV})^{2}$, corresponding to $\alpha_{s}\left(Q^{2}\right)=0.087,0.069$, and $0.058 .{ }^{15}$ For each value of $Q^{2}$, we calculate the expected NLL function $\tau g(\tau)$, Eqs. (221) and (222). Then we plot the ratio

$$
R\left(\tau, Q^{2}\right)=\frac{\left(\tau / \sigma_{\mathrm{H}}\right) d \sigma / d \tau}{\tau g(\tau)}
$$

The results are displayed in Fig. 9. In the case $Q^{2}=(100 \mathrm{TeV})^{2}$, there are typically around 100 partons produced in each event. This causes DEDUCTOR to operate very slowly, which leads to substantial statistical fluctuations that are visible in the plot.

If the log summation is working at the NLL level, the ratio plotted should be close to 1 and should get closer to 1 as $Q^{2}$ increases. We note two features of the results. First, for any fixed value of $Q^{2}, \tau g(\tau)$ fails to match the parton shower result for sufficiently small $\tau$. The value of $\tau$ at which this failure sets in decreases as $Q^{2}$ grows. For larger values of $\tau$, but still with $\tau<0.1, R\left(\tau, Q^{2}\right)$ is approximately constant:

$$
R\left(\tau, Q^{2}\right) \approx R_{0}\left(Q^{2}\right)
$$

\footnotetext{
${ }^{15}$ Reference [16] considers $\alpha_{s}\left(Q^{2}\right)$ as small as 0.005 , corresponding to $Q^{2} \approx\left(10^{70} \mathrm{GeV}\right)^{2}$ but DEDUCTOR is not capable of working with values of $Q^{2}$ as large as this.
} 


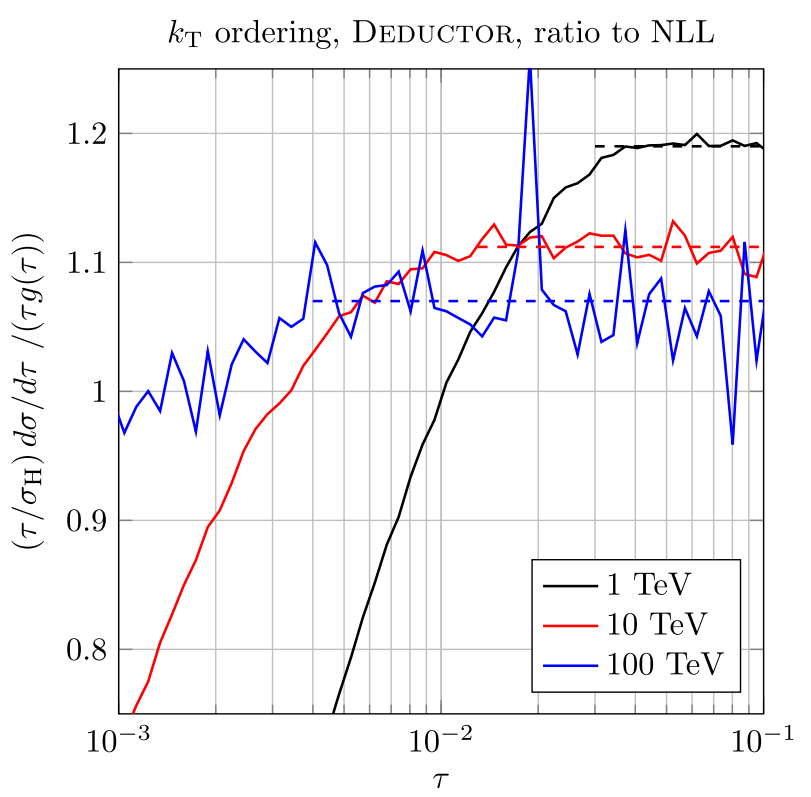

FIG. 9. Ratio of $\left(\tau / \sigma_{\mathrm{H}}\right) d \sigma / d \tau$ with $k_{\mathrm{T}}$ ordering to the NLL expectation, $\tau g(\tau)$, Eqs. (221) and (222). The ratio is calculated at $Q^{2}=(1 \mathrm{TeV})^{2}, Q^{2}=(10 \mathrm{TeV})^{2}$, and $Q^{2}=(100 \mathrm{TeV})^{2}$. We use a cutoff on the transverse momentum in splittings: $k_{\mathrm{T}}>$ $1 \mathrm{GeV}$ in each case.

These values $\left(R_{0}=1.190,1.112,1.070\right)$ are shown as dashed lines in Fig. 9. Second, we note that $R\left(\tau, Q^{2}\right)$ is fairly close to 1 and gets closer to 1 as $Q^{2}$ increases. In fact, to within about $10 \%$,

$$
R_{0}\left(Q^{2}\right)-1 \approx 23 \alpha_{s}^{2}\left(Q^{2}\right)
$$

This is consistent with the expectation that $R_{0}\left(Q^{2}\right) \rightarrow 0$ as $\alpha_{\mathrm{s}}\left(Q^{2}\right) \rightarrow 0$. We tentatively conclude from these results that the $k_{\mathrm{T}}$-ordered DEDUCTOR shower is correctly summing thrust logarithms at the NLL level, even though the difference between the shower result and the NLL analytical result is larger for $k_{\mathrm{T}}$ ordering than for $\Lambda$ ordering.

\section{EFFECT OF THE MOMENTUM MAPPING FOR $\Lambda$ ORDERING}

Recall from Sec. XVIII that in a splitting $p_{l} \rightarrow \hat{p}_{l}+\hat{p}_{m+1}$, we always have $p_{l} \neq \hat{p}_{l}+\hat{p}_{m+1}$. In order to conserve momentum, we need to map the momenta $p_{i}$ into new momenta $\hat{p}_{i}$ such that

$$
\sum_{i=1}^{m+1} \hat{p}_{i}=\sum_{i=1}^{m} p_{i}
$$

In the DEDUCTOR algorithm, this is accomplished by using a Lorentz transformation [19]

$$
\hat{p}_{i}^{\mu}=\Lambda_{\nu}^{\mu} p_{i}^{\nu}, \quad i \notin\{l, m+1\} .
$$

The Lorentz transformation is defined to be a boost in the plane of $p_{l}$ and $Q$. We have found in Sec. XVIII that the boost angle $\omega$ is small, of order $y$, and that the effect of this small Lorentz transformation on the thrust is small compared to the order $y$ effect produced by the splitting itself.

For any parton shower, one will need a momentum mapping that preserves the total momentum. The global mapping produced by a Lorentz transformation is not the only possibility. A more widely used local choice is provided by the Catani-Seymour dipole splitting formalism [44] or the local mapping in PYTHIA [45]. For the CataniSeymour choice, we start with the parton $l$ that splits and its dipole partner $k$, with momenta $p_{l}$ and $p_{k}$. After the splitting, we have a new parton $m+1$ and new momenta $\hat{p}_{i}, \hat{p}_{m+1}$ and $\hat{p}_{k}$. The definition is

$$
\begin{aligned}
\hat{p}_{m+1} & =(1-z) p_{l}+z y p_{k}+k_{\perp}, \\
\hat{p}_{l} & =z p_{l}+(1-z) y p_{k}-k_{\perp}, \\
\hat{p}_{k} & =(1-y) p_{k},
\end{aligned}
$$

with $k_{\perp} \cdot p_{j}=k_{\perp} \cdot p_{k}=0$. Here $z, y$, and $k_{\perp}$ are different from $z, y$ and $k_{\perp}$ defined for DEDUCTOR kinematics. The momenta of the other partons are unchanged:

$$
\hat{p}_{i}=p_{i} \quad i \notin\{l, k, m+1\} .
$$

With this definition,

$$
p_{l}+p_{k}=\hat{p}_{l}+\hat{p}_{m+1}+\hat{p}_{k}
$$

Thus the total momentum is conserved. We have $\hat{p}_{m+1}+$ $\hat{p}_{l}=p_{l}+y p_{k}$ so

$$
y=\frac{\hat{p}_{l} \cdot \hat{p}_{m+1}}{p_{l} \cdot p_{k}} .
$$

From $\hat{p}_{m+1}^{2}=0$ we derive

$$
-k_{\perp}^{2}=z(1-z) y 2 p_{l} \cdot p_{k} .
$$

Note that if we start with a two parton state, $m=2$, and let one of the two partons, $l$, split to produce parton $m+1$, then there is precisely one parton $i$ with $i \notin\{l, m+1\}$ in Eq. (273) and this is the same as parton $k$ in Eq. (274). That is, the global and local mappings are the same for $\mathcal{S}_{\mathcal{Y}}^{[1]}\left(\mu^{2} ; \nu\right)$ for $m=2$. The operators $\mathcal{S}_{\mathcal{Y}}^{[k]}\left(\mu^{2} ; \nu\right)$, with $k$ real or virtual splittings, do depend on the choice of momentum mapping for $k \geq 2$.

The local momentum mapping has a feature for thrust that one might regard as peculiar. Suppose that parton $l$ is in the right thrust hemisphere, $l \in \mathrm{R}$. Then for a small angle splitting, the daughter partons $l$ and $m+1$ will also be in the right hemisphere. In the case that $k \in \mathrm{R}$, we split a dipole that is entirely in $R$. Then Eqs. (275) and (276) imply 
that both $\tau_{\mathrm{R}}$ and $\tau_{\mathrm{L}}$ in Eq. (124) are unchanged by the splitting, so that $\tau=\tau_{\mathrm{R}}+\tau_{\mathrm{L}}$ is unchanged. Since, in this class of choices for the dipole that splits, the thrust is not changed, the real-virtual cancelation between $\mathcal{S}^{[1,0]}\left(\mu^{2}\right)$ and $\mathcal{S}^{[0,1]}\left(\mu^{2}\right)$ simply removes contributions of these dipoles from the calculation of the thrust distribution.

With $\Lambda$ ordering and a local momentum mapping, the argument in Sec. XXI that the shower sums logarithms of thrust at the LL level still works, but the argument in Appendix A for cancellations at the NLL level fails. Thus we cannot expect a $\Lambda$-ordered parton shower that uses a local momentum mapping following Eqs. (275) and (276) to properly sum the logarithms of $\nu$ at NLL accuracy.

We can check what happens numerically by calculating $\left\langle\mathcal{I}_{2}^{[2]}(\nu)\right\rangle$, Eq. (248), using the $\Lambda$-ordered Deductor parton shower algorithm with exact color but with the CataniSeymour momentum mapping substituted for the global momentum mapping. The result is shown as the solid red curve in Fig. 10. We note immediately that this result is completely different from the result in Fig. 1: in the range $\log (\nu)<8,\left|\left\langle\mathcal{I}_{2}^{[2]}(\nu)\right\rangle\right|$ with the global momentum mapping is less than 1 while with the local mapping it reaches values greater than 30. Leaving aside the magnitude of $\left\langle\mathcal{I}_{2}^{[2]}(\nu)\right\rangle$, if the parton shower algorithm with a local momentum mapping produced NLL accuracy for summing $\log (\nu)$ factors, the graph of $\left\langle\mathcal{I}_{2}^{[2]}(\nu)\right\rangle$ would be a straight line, but it is not. The dashed blue curve is $d\left\langle\mathcal{I}_{2}^{[2]}(\nu)\right\rangle / d \log (\nu)$. This curve is not a constant but rather a straight line.

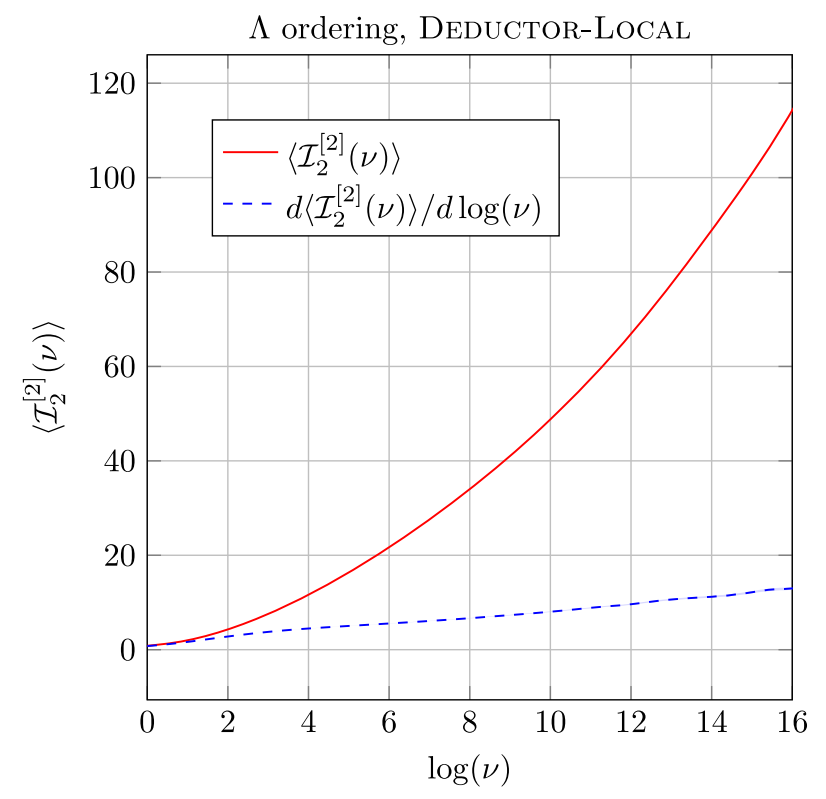

FIG. 10. Plot of $\left\langle\mathcal{I}_{2}^{[2]}(\nu)\right\rangle$, as in Fig. 1, for the DeduCtor splitting functions with the Catani-Seymour local momentum mapping [44]. $\left\langle\mathcal{I}_{2}^{[2]}(\nu)\right\rangle$ is approximately quadratic in $\log (\nu)$, indicating that $\mathcal{I}_{2}^{[2]}(\nu)$ changes the NLL result.
This implies that at $\operatorname{large} \log (\nu),\left\langle\mathcal{I}_{2}^{[2]}(\nu)\right\rangle$ is has contributions up to $\log ^{2}(\nu)$.

We conclude from the combination of the analytical argument and the numerical results that using a local momentum mapping destroys the NLL accuracy of the result from a $\Lambda$-ordered parton shower, although LL accuracy is maintained.

\section{LOCAL MOMENTUM MAPPING WITH OTHER ORDERINGS}

As we have seen in Sec. XXVII, a parton shower algorithm needs to conserve momentum while accommodating the approximation that a parton that splits to two partons was on shell before the splitting. DEDUCTOR uses a global recoil strategy that spreads the needed momentum over all of the other partons in the event. With a local momentum mapping in the style of Catani-Seymour, Eq. (274), the recoil momentum is taken up by a single parton, possibly a very soft parton. For this reason the global recoil strategy seems less likely to lead to problems than the local recoil strategy.

Nevertheless, a local momentum mapping can certainly work. Indeed, we present an argument in Appendix B that $\mathcal{I}_{2}^{[2]}(\nu)$ in DEDUCTOR with $k_{\mathrm{T}}$ ordering is well behaved. In this construction, the local and global momentum mappings were equivalent in the limits considered. Thus $\mathcal{I}_{2}^{[2]}(\nu)$ with $k_{\mathrm{T}}$ ordering and a local momentum mapping should be well behaved.

We can investigate this issue by calculating $\left\langle\mathcal{I}_{2}^{[2]}(\nu)\right\rangle$ using two shower algorithms with a local momentum mapping following Eq. (274). The algorithms we use follow closely the PANLOCAL shower of Ref. [16], but with color treated exactly. In the first algorithm that we use, the parameter $\beta$ that defines the ordering variable in the PanLocal algorithm is set to $\beta=0$. That corresponds to $k_{\mathrm{T}}$ ordering. In the second algorithm, we choose $\beta=0.5$. Roughly, that is half way between $k_{\mathrm{T}}$ ordering and $\Lambda$ ordering. Reference [16] claims that these PANLOCAL showers sum the trust distribution at NLL accuracy at leading color.

The results are shown in Figs. 11 and 12. In each case, in the range $\log (\nu)<8,\left|\left\langle\mathcal{I}_{2}^{[2]}(\nu)\right\rangle\right|$ reaches values greater than 10, while for DEDUCTOR with $\Lambda$ ordering this same quantity is less than 1 . Nevertheless, in each case, we see that $\left\langle\mathcal{I}_{2}^{[2]}(\nu)\right\rangle$ is, to a good approximation, a linear function of $\log (\nu)$ for $\operatorname{large} \log (\nu)$. This is consistent with NLL accuracy for summing logarithms of $\nu$.

In Figs. 13 and 14, we plot $\left\langle\mathcal{I}_{3}^{[2]}(\nu)\right\rangle$ for the two PANLOCAL shower algorithms. To be consistent with NLL accuracy, $\left\langle\mathcal{I}_{3}^{[2]}(\nu)\right\rangle$ at large $\log (\nu)$ should not contain terms $\log ^{j}(\nu)$ for $j=3$ or higher. The numerical results are consistent with this NLL expectation. In fact, in each case 


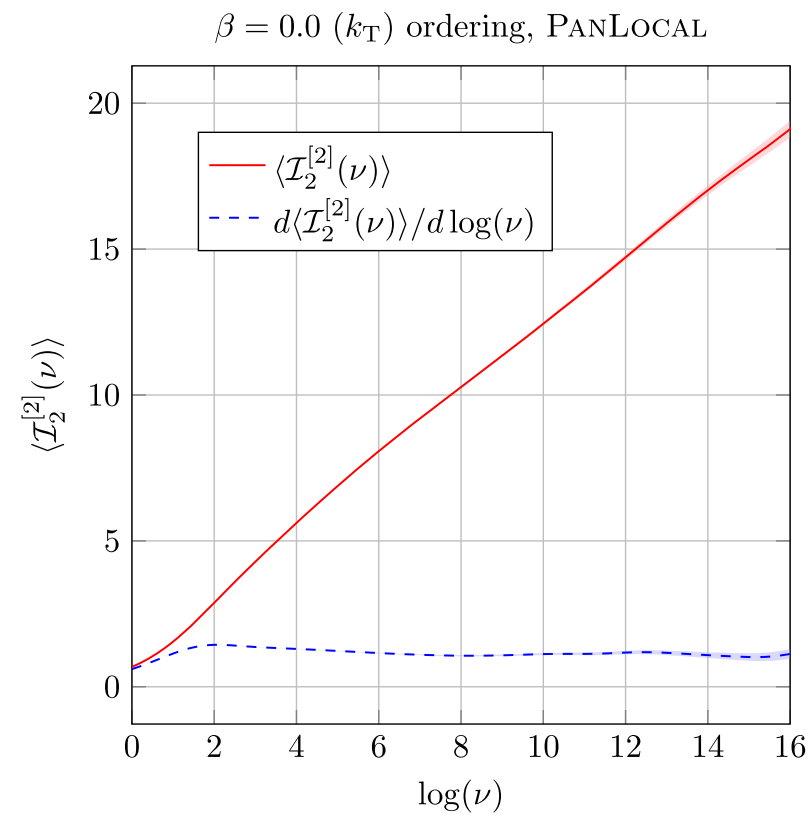

FIG. 11. Plot of $\left\langle\mathcal{I}_{2}^{[2]}(\nu)\right\rangle$, as in Fig. 1, for a shower with $k_{\mathrm{T}}$ $(\beta=0.0)$ ordering and the Catani-Seymour local momentum mapping [44] according to an algorithm based on the PANLOCAL dipole shower of Ref. [16] with exact color. For large $\log (\nu)$, $\left\langle\mathcal{I}_{2}^{[2]}(\nu)\right\rangle$ is approximately linear in $\log (\nu)$, indicating that $\mathcal{I}_{2}^{[2]}(\nu)$ leaves the NLL result intact.

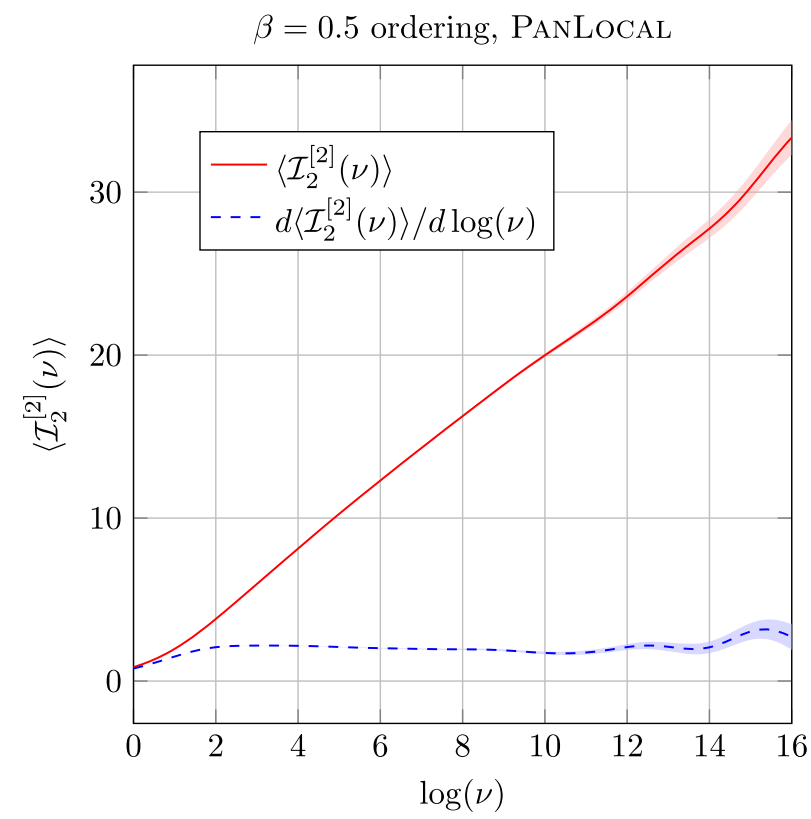

FIG. 12. Plot of $\left\langle\mathcal{I}_{2}^{[2]}(\nu)\right\rangle$, as in Fig. 1, versus $\log (\nu)$, for a shower with $\beta=0.5$ ordering and the Catani-Seymour local momentum mapping [44] according to an algorithm based on the PANLOCAL dipole shower of Ref. [16] with exact color. For large $\log (\nu),\left\langle\mathcal{I}_{2}^{[2]}(\nu)\right\rangle$ is approximately linear in $\log (\nu)$, indicating that $\mathcal{I}_{2}^{[2]}(\nu)$ leaves the NLL result intact.

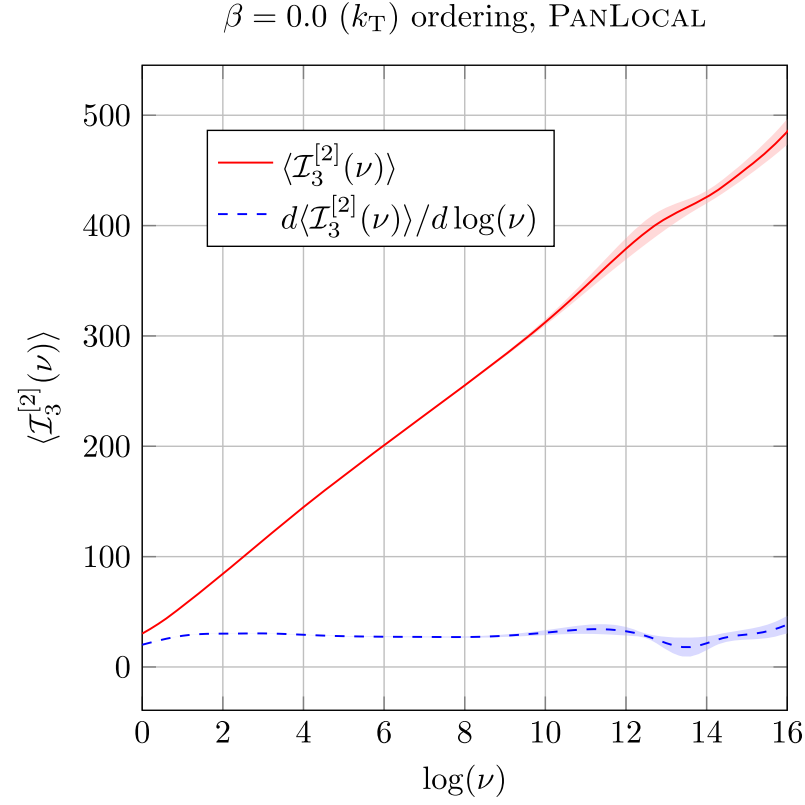

FIG. 13. Plot of $\left\langle\mathcal{I}_{3}^{[2]}(\nu)\right\rangle$, as in Fig. 2, for a shower with $k_{\mathrm{T}}$ $(\beta=0.0)$ ordering and the Catani-Seymour local momentum mapping [44] according to an algorithm based on the PANLOCAL dipole shower of Ref. [16] with exact color.

the highest power of $\log (\nu)$ numerically is $\log ^{1}(\nu)$. The coefficient of $\log ^{2}(\nu)$ vanishes to a good approximation. This tells us that the average value of the scale of the coupling inside the integrations is about $Q^{2} / \nu$.

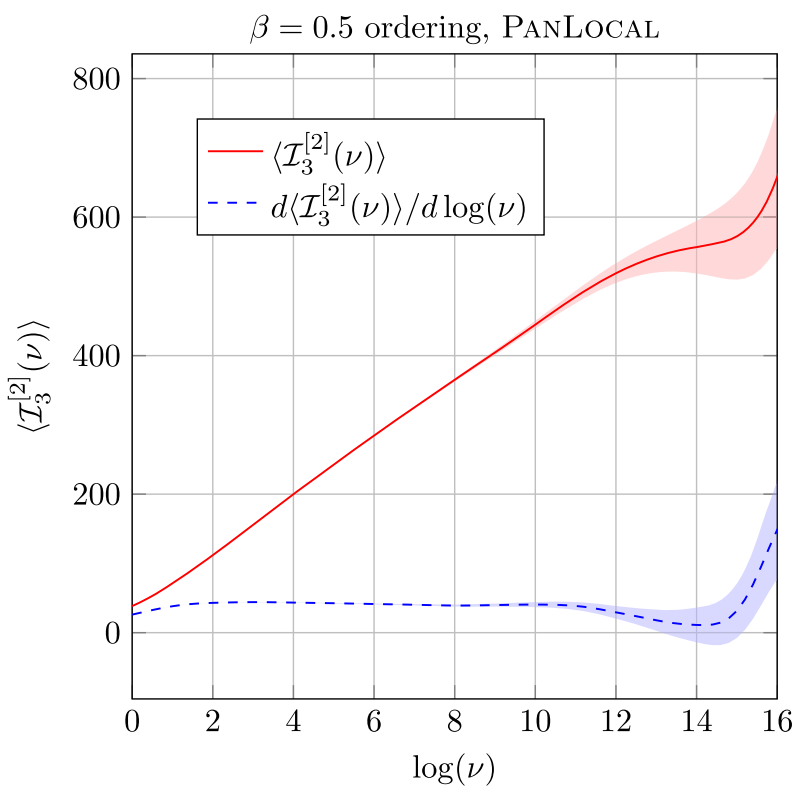

FIG. 14. Plot of $\left\langle\mathcal{I}_{3}^{[2]}(\nu)\right\rangle$, as in Fig. 2, versus $\log (\nu)$ for a shower with $\beta=0.5$ ordering and the Catani-Seymour local momentum mapping [44] according to an algorithm based on the PANLOCAL dipole shower of Ref. [16] with exact color. 


\section{CONCLUSIONS}

In Secs. II through X, we presented a general program for gaining direct access to how a parton shower sums large logarithms in either hadron-hadron, electron-hadron, or electron-positron collisions. We provided some summarizing remarks on the general program in Sec. X. In Secs. XI through XXVIII, we applied this program to electronpositron annihilation. We have limited ourselves to just one example, the thrust distribution. We have, however, looked at results for more than one shower algorithm.

The method that we propose works with the appropriate integral transform of the distribution of interest. In this case, we need the Laplace transform $\tilde{g}(\nu)$, Eq. (126), of the thrust distribution. We seek to find how $\tilde{g}(\nu)$ behaves for large $\nu$.

We rearrange the cross section calculation so as to write $\tilde{g}(\nu)$ in the form from Eq. (231),

$$
\tilde{g}(\nu)=\exp \left(\left(1|\mathcal{I}(\nu)|\{p, f, c, c\}_{2}\right)\right) .
$$

Here $\left.\mid\{p, f, c, c\}_{2}\right)$ is a color and flavor singlet $q \bar{q}$ basis state with $p_{1}+p_{2}=Q$ and the operator $\mathcal{I}(\nu)$ is an integral,

$$
\mathcal{I}(\nu)=\int_{\mu_{f}^{2}}^{Q^{2}} \frac{d \bar{\mu}^{2}}{\bar{\mu}^{2}} \mathcal{S}_{\mathcal{Y}}\left(\bar{\mu}^{2} ; \nu\right) .
$$

We expand $\mathcal{I}(\nu)$ in powers of the shower evolution operator $\mathcal{S}\left(\mu^{2}\right)$. Then the coefficients $\mathcal{I}^{[k]}(\nu)$, proportional to $k$ powers of $\mathcal{S}\left(\mu^{2}\right)$, can be further expanded as

$$
\mathcal{I}^{[k]}(\nu)=\sum_{n=k}^{\infty}\left[\frac{\alpha_{s}\left(Q^{2} / \nu\right)}{2 \pi}\right]^{n} \mathcal{I}_{n}^{[k]}(\nu)
$$

in which the strong coupling is evaluated at a fixed scale $Q^{2} / \nu$. Thus the shower result is quite directly expressed in exponentiated form in terms of an operator $\mathcal{I}(\nu)$ with a known perturbative expansion.

For the DEDUCTOR shower algorithm with either $\Lambda$ or $k_{\mathrm{T}}$ ordering, $\mathcal{I}^{[1]}(\nu)$ provides the standard NLL summation of $\log (\nu)$ factors. ${ }^{16}$ In order for the contributions $\mathcal{I}_{n}^{[k]}(\nu)$ for $k \geq 2$ to not spoil the NLL summation, $\mathcal{I}_{n}^{[k]}(\nu)$ should not contain more than $n-1$ powers of $\log (\nu)$.

For the DEDUCTOR shower algorithm with its default $\Lambda$ ordering, we find analytically that $\mathcal{I}_{n}^{[k]}(\nu)$ does not contain more than $n-1$ powers of $\log (\nu)$.

We have no such result for DEDUCTOR with $k_{\mathrm{T}}$ ordering, but we outline an argument in Appendix B that real-virtual cancellations in $\mathcal{I}_{2}^{[2]}(\nu)$ reduce its large $\nu$ behavior from $\log ^{4}(\nu)$ to $\log ^{1}(\nu)$.

\footnotetext{
${ }^{16}$ The current DEDUCTOR code with $\Lambda$ ordering, as distinct from the algorithm that it is based on, lacks the term with coefficient $\beta_{1}$ needed to evaluate the dependence of $\alpha_{\mathrm{s}}((1-$ $\left.z) y Q^{2}\right)$ on $(1-z)$. This changes the DEDUCTOR result at the NLL level.
}

We evaluate $\mathcal{I}_{2}^{[2]}(\nu)$ numerically. In order not to spoil NLL summation, its large $\nu$ behavior should be no more than $\log ^{1}(\nu)$. For the DEDUCTOR algorithm with $\Lambda$ ordering but with a local momentum mapping instead of the global momentum mapping used in DEDUCTOR, we find $\log ^{2}(\nu)$ behavior, implying a failure of NLL accuracy (Fig. 10). In other cases, we find $\log ^{1}(\nu)$ behavior, consistently with NLL accuracy. These cases include DEDUCTOR- $\Lambda$ (Fig. 1), Deductor- $k_{\mathrm{T}}$ (Fig. 6), PANLocal- $(\beta=0)$ (Fig. 11), and PANLOCAL- $(\beta=0.5)$ (Fig. 12).

We also evaluate $\mathcal{I}_{3}^{[2]}(\nu)$ numerically for the shower algorithms Deductor- $\Lambda$ (Fig. 2), Deductor- $k_{\mathrm{T}}$ (Fig. 7), PANLOCAL- $(\beta=0)$ (Fig. 13), and PANLOCAL- $(\beta=0.5)$ (Fig. 14). In each case, we find $\operatorname{large} \log (\nu)$ behavior with no more than 2 powers of $\log (\nu)$, consistently with NLL accuracy.

We emphasize in this paper writing the appropriate integral transform of the distribution of interest, such as the thrust distribution, as an exponential and examining the exponent $\mathcal{I}(\nu)$. However, it is also possible to simply look directly at the distribution of interest as it is generated by a given parton shower. For this, one needs to simulate collisions at large values of $Q^{2}$. We have not pushed this method to nearly as large a value of $Q^{2}$ as in Ref. [16]. However, we find that, at least for electron-positron annihilation, this direct method can be useful.

Specifically, we examine directly the thrust distribution $\tau g(\tau)$ for DEDUCTOR with $\Lambda$ and $k_{\mathrm{T}}$ ordering, using $Q^{2}=(10 \mathrm{TeV})^{2}$. With $\Lambda$ ordering, this works well (Figs. 3 and 4). With $k_{\mathrm{T}}$ ordering (Fig. 8), the agreement with the analytic NLL expectation is not as good. However, when we compare $\tau g(\tau)$ to the NLL expectation at a sequence of values of $Q^{2}$, we find what appears to be convergence to the NLL result as $Q^{2}$ increases (Fig. 9).

For both analytical and numerical analyses of $\mathcal{I}_{n}^{[k]}(\nu)$, we have used exact QCD color. For direct calculations of the thrust distribution using DEDUCTOR we have used the LC+ approximation for color. We have seen in Fig. 4 that the LC+ approximation is numerically very accurate for the thrust distribution, although we have noted in Sec. XXV that the LC+ approximation may change the coefficients of some $\log (\nu)$ factors in $\mathcal{I}_{n}^{[k]}(\nu)$ from what they are with full color. We have also seen in Sec. XXV that the use of just the leading color approximation can lead to loss of accuracy if one does not carefully adjust the choice between $C_{\mathrm{F}}$ and $C_{\mathrm{A}} / 2$, as studied in Ref. [17].

There are several avenues available for future research that extends the results of this paper.

First, the method of this paper applies to several observables in electron-positron annihilation. We have tried variations on the shower algorithm examined, but have looked at only one observable, the thrust distribution. 
It is certainly worthwhile to see what patterns emerge if we look at other observables.

Second, the method developed in Secs. II through X applies to observables in hadron-hadron collisions as well as in electron-positron collisions. It is of interest to see how this method works in practice for some hadron-hadron observables, starting with the $k_{\mathrm{T}}$ distribution in the DrellYan process.

Third, we construct numerical implementations of $\mathcal{I}_{2}^{[2]}(\nu)$ and $\mathcal{I}_{3}^{[2]}(\nu)$ for the particular observable examined and for several shower algorithms. This allows one to test numerically if the large $\nu$ behaviors of $\mathcal{I}_{2}^{[2]}(\nu)$ and $\mathcal{I}_{3}^{[2]}(\nu)$ are consistent with NLL summation. When we find for a certain shower algorithm that NLL summation fails at the level of $\mathcal{I}_{2}^{[2]}(\nu)$ or $\mathcal{I}_{3}^{[2]}(\nu)$, then NLL summation fails for that shower algorithm and observable. However, if NLL summation is not spoiled by $\mathcal{I}_{2}^{[2]}(\nu)$ or $\mathcal{I}_{3}^{[2]}(\nu)$, it could still fail in $\mathcal{I}_{n}^{[k]}(\nu)$ for some larger values of $k$ and $n$. Thus it would be valuable to have numerical implementations of $\mathcal{I}_{n}^{[k]}(\nu)$ for some larger values of $k$ and $n$. Then one would have more stringent numerical tests of NLL summation for a given shower algorithm and a given observable.

Fourth, it would be helpful to have analytical insight into the behavior of the operators $\mathcal{I}^{[k]}(\nu)$ for $k \geq 3$ in cases that are similar to the thrust distribution using a $k_{\mathrm{T}}$-ordered shower.

Fifth, it would be worthwhile to examine in detail the effect of using the LC+ approximation for color instead of exact color for maintaining LL or NLL summation of large logarithms.

Sixth, although the LC+ approximation for color is numerically quite accurate in cases like that exhibited in Fig. 4, we have seen in Eq. (266) that it leaves out some contributions that are potentially important. Thus it would be worthwhile to find an improved approximation for color in a parton shower.

We close with the observation that it is expecting a lot to expect that a first order shower algorithm will sum logarithms at the LL or NLL level. If we had a parton shower based on splitting functions at order $\alpha_{s}^{N}$ [18], then we could expect to correctly produce contributions to $\mathcal{I}(\nu)$ of order $\alpha_{s}^{n} \log ^{j}(\nu)$ with $n \leq N, j \leq n+1$. We might not correctly produce contributions of order $\alpha_{s}^{n} \log ^{j}(\nu)$ with $n>N, j \leq n+1$ because we lack the order $\alpha_{s}^{n}$ contributions to the shower splitting functions. However, contributions of order $\alpha_{s}^{n} \log ^{j}(\nu)$ with $j>n+1$ should vanish because these contributions can never be provided by $\alpha_{s}^{n}$ contributions to the shower splitting functions. Currently, all that we have (in several variations) is a first order shower, $N=1$. Thus we can expect to correctly produce contributions of order $\alpha_{s}^{1} \log ^{2}(\nu)$ and $\alpha_{s}^{1} \log ^{1}(\nu)$. We can also expect to obtain exponentiation of logarithms of $\nu$ : contributions of order $\alpha_{s}^{n} \log ^{j}(\nu)$ with $j>n+1$ should vanish. With care, we can hope to have LL or NLL summation of $\log (\nu)$ factors, but this relies on incorporating the most important parts of higher order splitting operators into the first order operator $\mathcal{S}$.

\section{ACKNOWLEDGMENTS}

We thank the participants in the conference PSR21Parton Showers and Resummation for useful discussions. This work was supported in part by the United States Department of Energy under Grant No. DE-SC0011640. The work benefited from access to the University of Oregon high performance computer cluster, Talapas.

\section{APPENDIX A: STRUCTURE OF $\mathcal{S}_{\mathcal{Y}}$ AT NLL ACCURACY}

We examine $\mathcal{S}_{\mathcal{Y}}^{[k]}\left(x Q^{2} / \mu\right)$ for $x$ of order 1 and $k \geq 2$. We prove that this operator has at most $n-1$ factors of $\log (\nu)$ at order $\alpha_{s}^{n}\left(Q^{2} / \nu\right)$.

Recall from Sec. XXI that $\mathcal{Y}^{[k]}\left(x Q^{2} / \mu\right)$ for $x$ of order 1 and $k \geq 2$ has at most $n$ factors of $\log (\nu)$ at order $\alpha_{s}^{n}\left(Q^{2} / \nu\right)$.

We also note that $\mathcal{S}_{l}^{[1,0]}\left(x Q^{2} / \nu\right)$ for $x$ of order 1 has one power of $\log (\nu)$ at order $\alpha_{s}\left(Q^{2} / \nu\right)$, where the $\log (\nu)$ factor arises from an integration $d(1-z) /(1-z)$ down to a lower limit proportional to $1 / \nu$, as in Eq. (215). Thus $\mathcal{S}_{l}^{[1,0]}\left(x Q^{2} / \nu\right)$ for $x$ of order 1 has at most $n$ powers of $\log (\nu)$ at order $\alpha_{s}^{n}\left(Q^{2} / \nu\right)$.

To proceed, we prove that $\mathcal{S}_{\mathcal{Y}}^{[k]}\left(x Q^{2} / \mu\right)$ with $k=2$ contains at most $n-1$ factors of $\log (\nu)$ at order $\alpha_{s}^{n}\left(Q^{2} / \nu\right)$ and we prove that if this property holds for $k=2,3, \ldots, N$, then it holds for $k=N+1$.

Consider Eq. (245) for $\mathcal{S}_{\mathcal{Y}}^{[k+1]}\left(x Q^{2} / \nu ; \nu\right)$ for $k \geq 2$. In the first term, at order $\alpha_{s}^{k+1}\left(Q^{2} / \nu\right)$, there are $k$ powers of $\log (\nu)$ from $\mathcal{Y}^{[k]}$ and one power from $\mathcal{S}_{l}^{[1,0]}$. In the second term (if $k \geq 3)$ at order $\alpha_{s}^{k+1}\left(Q^{2} / \nu\right)$ there are there are $k+1-j$ powers of $\log (\nu)$ from $\mathcal{Y}^{[k+1-j]}$ and $j-1$ powers from $\mathcal{S}_{\mathcal{Y}}^{[j]}$, for a total of just $k$ powers of $\log (\nu)$. That is, this contribution is NNLL. In the third term, at order $\alpha_{s}^{k+1}\left(Q^{2} / \nu\right)$ there is one power of $\log (\nu)$ from $\mathcal{S}_{l}^{[1,0]}$ and $k-1$ powers of $\log (\nu)$ from $\mathcal{S}_{\mathcal{Y}}^{[k]}$, for a total of $k$ powers of $\log (\nu)$. That is, this contribution is NNLL. If we expand the NNLL contributions to higher order in $\alpha_{s}\left(Q^{2} / \nu\right)$, we add just one power of $\log (\nu)$ per $\alpha_{s}$, so the contributions remain NNLL. This gives us

$$
\begin{aligned}
& \mathcal{S}_{\mathcal{Y}}^{[k+1]}\left(x Q^{2} / \nu ; \nu\right) \\
& =\sum_{l}\left[\mathcal{Y}^{[k]}\left(x Q^{2} / \nu ; \nu\right)\left[\mathcal{S}_{l}^{[1,0]}\left(x Q^{2} / \nu\right)\right]_{1-\mathbb{P}}\right]_{\mathbb{P}} e^{-\xi_{l}^{\mathrm{op}} x} \\
& \quad+\mathrm{NNLL} .
\end{aligned}
$$

This leaves us with an NLL contribution if the NLL contribution does not cancel. This result does not include 
$\mathcal{S}_{\mathcal{Y}}^{[2]}$. For $\mathcal{S}_{\mathcal{Y}}^{[2]}$, Eq. (239) gives us 2 powers of $\log (\nu)$ at order $\alpha_{s}^{2}\left(Q^{2}\right)$. This is an NLL contribution if the NLL contribution does not cancel.

If we use Eq. (A1), then we need information on $\mathcal{Y}^{[k]}$. We can use Eq. (247) for $\mathcal{Y}^{[k+1]}\left(x Q^{2} / \nu ; \nu\right)$ for $k \geq 1$. In the first term at order $\alpha_{s}^{k+1}\left(Q^{2} / \nu\right)$ there are $k$ powers of $\log (\nu)$ from $\mathcal{Y}^{[k]}$ and one power of $\log (\nu)$ from $\mathcal{S}_{l}^{[1,0]}$, giving us a total of $k+1$ powers of $\log (\nu)$. This is an NLL contribution. In the second term (for $k \geq 2$ ) at order $\alpha_{s}^{k+1}\left(Q^{2} / \nu\right)$ there are $k+1-j$ powers of $\log (\nu)$ from $\mathcal{Y}^{[k+1-j]}$ and $j-1$ powers of $\log (\nu)$ from $\mathcal{S}_{\mathcal{Y}}^{[j]}$, giving us a total of $k$ powers of $\log (\nu)$. This is an NNLL contribution. In the third term at order $\alpha_{s}^{k+1}\left(Q^{2} / \nu\right)$ there are $k$ powers of $\log (\nu)$ from $\mathcal{S}_{\mathcal{Y}}^{k+1]}$. This is an NNLL contribution. Again, if we expand the NNLL contributions to higher order in $\alpha_{s}\left(Q^{2} / \nu\right)$, we add just one power of $\log (\nu)$ per $\alpha_{s}$, so the contributions remain NNLL. This leaves us with

$$
\mathcal{Y}^{[k+1]}\left(x Q^{2} / \nu ; \nu\right)=-\sum_{l} \int_{0}^{x} \frac{d x_{1}}{x_{1}} \mathcal{Y}^{[k]}\left(x_{1} Q^{2} / \nu ; \nu\right)\left[\mathcal{S}_{l}^{[1,0]}\left(x_{1} Q^{2} / \nu\right)\right]_{\mathbb{P}}\left(1-e^{-\xi_{l}^{\mathrm{op}} x_{1}}\right)+\text { NNLL. }
$$

This derivation does not include $\mathcal{Y}^{[1]}$. For $\mathcal{Y}^{[1]}$ we can use Eq. (237), which gives us just Eq. (A2) with $\mathcal{Y}^{[0]}$ replaced by 1 and no NNLL additional contribution.

Equation (A2) gives us a recursion relation that we can solve to NLL accuracy in the form

$$
\begin{aligned}
\mathcal{Y}^{[k]}\left(x Q^{2} / \nu ; \nu\right)= & (-1)^{k} \sum_{l_{1} \ldots l_{k}} \int_{0}^{x} \frac{d x_{1}}{x_{1}} \int_{0}^{x_{1}} \frac{d x_{2}}{x_{2}} \cdots \int_{0}^{x_{k-1}} \frac{d x_{k}}{x_{k}}\left[\mathcal{S}_{l_{k}}^{[1,0]}\left(x_{k} Q^{2} / \nu\right)\right]_{\mathbb{P}}\left(1-e^{-\xi_{l_{k}}^{\mathrm{op}} x_{k}}\right) \cdots \\
& \times\left[\mathcal{S}_{l_{2}}^{[1,0]}\left(x_{2} Q^{2} / \nu\right)\right]_{\mathbb{P}}\left(1-e^{-\xi_{l_{2}}^{\mathrm{op}} x_{2}}\right)\left[\mathcal{S}_{l_{1}}^{[1,0]}\left(x_{1} Q^{2} / \nu\right)\right]_{\mathbb{P}}\left(1-e^{-\xi_{l_{1}}^{\mathrm{op}} x_{1}}\right)+\text { NNLL. }
\end{aligned}
$$

We can substitute this solution for $\mathcal{Y}^{[k]}$ into Eq. (A1) to give us

$$
\begin{aligned}
\mathcal{S}_{\mathcal{Y}}^{[k+1]}\left(x_{0} Q^{2} / \nu ; \nu\right)= & (-1)^{k} \sum_{l_{0} \ldots l_{k}} \int_{0}^{x_{0}} \frac{d x_{1}}{x_{1}} \int_{0}^{x_{1}} \frac{d x_{2}}{x_{2}} \cdots \int_{0}^{x_{k-1}} \frac{d x_{k}}{x_{k}}\left[\left[\mathcal{S}_{l_{k}}^{[1,0]}\left(x_{k} Q^{2} / \nu\right)\right]_{\mathbb{P}}\left(1-e^{-\xi_{l_{n}}^{\mathrm{op}} x_{k}}\right) \ldots\right. \\
& \left.\times\left[\mathcal{S}_{l_{2}}^{[1,0]}\left(x_{2} Q^{2} / \nu\right)\right]_{\mathbb{P}}\left(1-e^{-\xi_{l_{2}}^{\mathrm{op}} x_{2}}\right)\left[\mathcal{S}_{l_{1}}^{[1,0]}\left(x_{1} Q^{2} / \nu\right)\right]_{\mathbb{P}}\left(1-e^{-\xi_{l_{1}}^{\mathrm{op}} x_{1}}\right)\left[\mathcal{S}_{l_{0}}^{[1,0]}\left(x_{0} Q^{2} / \nu\right)\right]_{1-\mathbb{P}}\right]_{\mathbb{P}} e^{-\xi_{l_{0}}^{\mathrm{op} x_{0}}} \\
& +\mathrm{NNLL} .
\end{aligned}
$$

The explicit exponential factors restrict the $x_{i}$ integrations to $x_{i}$ of order 1 (as we have already seen). We now want to find how many factors of $\log (\nu)$ are contained in the operators $\mathcal{S}_{l}^{[1,0]}\left(x Q^{2} / \nu\right)$. Since $\log (x / \nu)$ is equivalent for this purpose to $\log (1 / \nu)$ when $x$ is of order 1 , we can replace all of the $x_{i}$ factors in the arguments of $\mathcal{S}_{l}^{[1,0]}\left(x Q^{2} / \nu\right)$ by 1 .

In Eq. (A4), we have factors $\exp \left(-\hat{\xi}_{l}^{\mathrm{op}} x_{i}\right)$. The parameters $\xi_{l}$, are defined in Eq. (204). They are close to $1: \xi_{l}-1$ is proportional to $\left[1-\cos \left(\theta\left(l, \vec{n}_{\mathrm{T}}\right)\right)\right]$. It is a good approximation to take the thrust axis $\vec{n}_{\mathrm{T}}$ to be the direction of either the quark or the antiquark in the $q-\bar{q}$ state at the start of the shower. Then the angle between $\vec{p}_{l}$ at a later stage of the shower and $\vec{n}$ is determined by the emission angles at the intervening stages. But in order to accumulate the maximal number of $\log (\nu)$ factors in these splittings, all of these emission angles must be small. That is, if we expand $\exp \left(-\xi_{l} x_{i}\right)$ in powers of $[1-\cos (\theta)]$, where $\theta$ is one of the splitting angles, then a factor $[1-\cos (\theta)]$ will eliminate a $\log (\nu)$ factor in an integration $d \cos (\theta) /[1-\cos (\theta)]$ with limits analogous to the limits in Eq. (114). We conclude that for the purpose of our present NLL calculation we can set all of the $\xi_{l}^{\text {op }}$ factors in Eqs. (A4) to 1 .

These changes gives us

$$
\begin{aligned}
\left.\mathcal{S}_{\mathcal{Y}}^{[k+1]}\left(x_{0} Q^{2} / \nu ; \nu\right) \mid\left\{p, f, c, c^{\prime}\right\}_{m}\right)= & (-1)^{k} \sum_{l_{0} \ldots l_{k}} \int_{0}^{x_{0}} \frac{d x_{1}}{x_{1}} \int_{0}^{x_{1}} \frac{d x_{2}}{x_{2}} \cdots \int_{0}^{x_{k-1}} \frac{d x_{k}}{x_{k}}\left[\left[\mathcal{S}_{l_{k}}^{[1,0]}\left(Q^{2} / \nu\right)\right]_{\mathbb{P}}\left(1-e^{-x_{k}}\right) \cdots\right. \\
& \left.\times\left[\mathcal{S}_{l_{2}}^{[1,0]}\left(Q^{2} / \nu\right)\right]_{\mathbb{P}}\left(1-e^{-x_{2}}\right)\left[\mathcal{S}_{l_{1}}^{[1,0]}\left(Q^{2} / \nu\right)\right]_{\mathbb{P}}\left(1-e^{-x_{1}}\right)\left[\mathcal{S}_{l_{0}}^{[1,0]}\left(Q^{2} / \nu\right)\right]_{1-\mathbb{P}}\right]_{\mathbb{P}} e^{-x_{0}} \\
& \left.\times \mid\left\{p, f, c, c^{\prime}\right\}_{m}\right)+\mathrm{NNLL} .
\end{aligned}
$$


The first $\mathcal{S}_{l}^{[1,0]}\left(Q^{2} / \nu\right)$ factor in Eq. (A5) is

$$
\left[\mathcal{S}_{l_{0}}^{[1,0]}\left(Q^{2} / \nu\right)\right]_{1-\mathbb{P}}=\mathcal{S}_{l_{0}}^{[1,0]}\left(Q^{2} / \nu\right)-\left[\mathcal{S}_{l_{0}}^{[1,0]}\left(Q^{2} / \nu\right)\right]_{\mathbb{P}} .
$$

The contribution from $\left[\mathcal{S}_{l_{0}}^{[1,0]}\left(Q^{2} / \nu\right)\right]_{\mathbb{P}}$ is rather simple and we will consider it later.

We begin by considering the contribution from $\mathcal{S}_{l_{0}}^{[1,0]}\left(Q^{2} / \nu\right)$. This operator, acting on the state $\left.\mid\left\{p, f, c, c^{\prime}\right\}_{m}\right)$, produces a linear combination of states with $m+1$ partons, $\left.\mid\left\{\hat{p}, \hat{f}, \hat{c}, \hat{c}^{\prime}\right\}_{m+1}\right)$,

$$
\begin{aligned}
\sum_{l_{0}=1}^{m} & \left.\mathcal{S}_{l_{0}}^{[1,0]}\left(Q^{2} / \nu\right) e^{-x_{0}} \mid\left\{p, f, c, c^{\prime}\right\}_{m}\right) \\
\approx & \left.-\sum_{\substack { l_{0}=1 \\
\begin{subarray}{c}{k_{0}=1 \\
k_{0} \neq l_{0}{ l _ { 0 } = 1 \\
\begin{subarray} { c } { k _ { 0 } = 1 \\
k _ { 0 } \neq l _ { 0 } } }\end{subarray}}^{m} \mathcal{C}_{0}\left(l_{0}, k_{0}\right) \mid\left\{c, c^{\prime}\right\}_{m}\right) \\
& \times \int \frac{d \phi_{0}}{2 \pi} \int \frac{d z_{0}}{1-z_{0}} \frac{\alpha_{s}\left(\lambda_{\mathrm{R}}\left(1-z_{0}\right) Q^{2} /\left(\nu a_{l_{0}}\right)\right)}{2 \pi} \\
& \times \Theta\left(\frac{a_{l_{0}}}{\nu \vartheta\left(l_{0}, k_{0}\right)}<1-z_{0}<1\right) \\
& \left.\times e^{-x_{0}} \mid\{\hat{p}, \hat{f}\}_{m+1}\right) .
\end{aligned}
$$

Here we use the approximate form of $\mathcal{S}^{[1,0]}\left(Q^{2} / \nu\right)$ given in Eq. (109). We split parton $l_{0}$ with dipole partner parton $k_{0}$, creating a new parton $m+1$, which we consider to be a gluon. The color operator is

$$
\mathcal{C}_{0}\left(l_{0}, k_{0}\right)=T_{l_{0}} \otimes T_{k_{0}}^{\dagger}+T_{k_{0}} \otimes T_{l_{0}}^{\dagger},
$$

as defined below Eq. (109). We have specified a scale argument based on the transverse momentum for the splitting for $\alpha_{\mathrm{s}}$. The new momentum $\hat{p}_{m+1}$ and the new momentum $\hat{p}_{l}$ are given by the splitting variables $y=1 / \nu$, $z_{0}$ and $\phi_{0}$. The new momenta $\hat{p}_{i}$ for $i \neq l_{0}, m+1$ are slightly different from the starting momenta, as specified by the momentum mapping.

Let us consider what the one of the operators, $\left[\mathcal{S}_{l_{i}}^{[1,0]}\left(Q^{2} / \nu\right)\right]_{\mathbb{P}}$, in Eq. (A5) does to this state. We consider the quantity

$$
\left.\left.\mid A_{i}\right)=\sum_{l_{i}=1}^{m+1}\left[\mathcal{S}_{l_{i}}^{[1,0]}\left(Q^{2} / \nu\right)\right]_{\mathbb{P}}\left(1-e^{-x_{i}}\right) \mid\left\{\hat{p}, \hat{f}, \hat{c}, \hat{c}^{\prime}\right\}_{m+1}\right) .
$$

Again, we use the approximate form of $\mathcal{S}^{[1,0]}\left(Q^{2} / \nu\right)$ given in Eq. (109), so that

$$
\begin{aligned}
\left.\mid A_{i}\right) \approx & -\sum_{l=1}^{m+1} \sum_{\substack{k=1 \\
k \neq l}}^{m+1} \mathcal{C}(l, k) \\
& \times \int \frac{d \phi}{2 \pi} \int \frac{d z}{1-z} \frac{\alpha_{s}\left(\lambda_{\mathrm{R}}(1-z) Q^{2} /\left(\nu \hat{a}_{l}\right)\right)}{2 \pi} \\
& \times \Theta\left(\frac{1}{\nu \hat{\vartheta}(l, k)}<\frac{1-z}{\hat{a}_{l}}<\frac{1}{\hat{a}_{l}}\right) \\
& \left.\times\left(1-e^{-x_{i}}\right) \mid\left\{\hat{p}, \hat{f}, \hat{c}, \hat{c}^{\prime}\right\}_{m+1}\right) .
\end{aligned}
$$

Here the hats in $\hat{\vartheta}(l, k)$ and $\hat{a}_{l}$ indicate that these quantities are based on the momenta in $\left.\mid\{\hat{p}, \hat{f}\}_{m+1}\right)$. In Eq. (A9), we split parton $l$ with dipole partner parton $k$, creating a new parton $m+2$, which we consider to be a gluon. ${ }^{17}$ However, the $[\cdots]_{\mathbb{P}}$ operation, Eqs. (157) and (158), returns us to the starting momentum and flavor state $\left.\mid\{\hat{p}, \hat{f}\}_{m+1}\right)$. With the $[\cdots]_{\mathbb{P}}$ operation, Eq. (159), the color operator is

$$
\begin{aligned}
\mathcal{C}(l, k) & =\left[T_{l} \otimes T_{k}^{\dagger}+T_{k} \otimes T_{l}^{\dagger}\right]_{\mathbb{P}} \\
& =1 \otimes \boldsymbol{T}_{l} \cdot \boldsymbol{T}_{k}+\boldsymbol{T}_{l} \cdot \boldsymbol{T}_{k} \otimes 1 .
\end{aligned}
$$

In the first term in the second line, the operator $\boldsymbol{T}_{l} \cdot \boldsymbol{T}_{k}$ operates on the bra color state and leaves the number of partons in the color state unchanged. The operator inserts a color matrix $T^{a}$ with gluon color index $a$ on line $l$ and another $T^{a}$ on line $k$. The dot in $\boldsymbol{T}_{k} \cdot \boldsymbol{T}_{l}$ indicates a sum over $a$. In the second term, the same operator is applied to the bra state.

There is an integration over the splitting variables $\phi$ and $z$. It will prove helpful to define a function $L(w, u)$ given by performing this integration,

$$
L(w, u)=\int_{0}^{2 \pi} \frac{d \phi}{2 \pi} \int_{1 / w}^{1 / u} \frac{d x}{x} \frac{\alpha_{s}\left(\lambda_{\mathrm{R}} x Q^{2} / \nu\right)}{2 \pi} .
$$

This function is to be expanded in powers of $\alpha_{s}\left(Q^{2} / \nu\right)$. At lowest order, this integration gives simply $\left[\alpha_{s} /(2 \pi)\right] \log (w / u)$. At higher orders in an expansion in powers of $\alpha_{s}\left(Q^{2} / \nu\right)$ the result is more complicated. With this notation,

$$
\begin{aligned}
\left.\mid A_{i}\right) \approx & -\sum_{l=1}^{m+1} \sum_{\substack{k=1 \\
k \neq l}}^{m+1} \mathcal{C}(l, k) L\left(\nu \hat{\vartheta}(l, k), \hat{a}_{l}\right) \\
& \left.\times\left(1-e^{-x_{i}}\right) \mid\left\{\hat{p}, \hat{f}, \hat{c}, \hat{c}^{\prime}\right\}_{m+1}\right) .
\end{aligned}
$$

We break up the sums in the form

\footnotetext{
${ }^{17}$ We omit splittings $\mathrm{g} \rightarrow q \bar{q}$ since these splittings lack a soft singularity. For a $q \rightarrow q \mathrm{~g}$ or $\bar{q} \rightarrow \bar{q} \mathrm{~g}$ splitting from an $m+1$ parton state, the daughter gluon is labeled $m+2$.
} 


$$
\begin{aligned}
\left.\mid A_{i}\right) \approx & -\left\{\sum_{\substack{l=1 \\
l \neq l_{0}}}^{m} \sum_{\substack{k=1 \\
k \neq l}}^{m+1} \mathcal{C}(l, k) L\left(\nu \hat{\vartheta}(l, k), \hat{a}_{l}\right)+\sum_{\substack{k=1 \\
k \neq l_{0}}}^{m} \mathcal{C}\left(l_{0}, k\right) L\left(\nu \hat{\vartheta}^{2}\left(l_{0}, k\right), \hat{a}_{l_{0}}\right)+\sum_{\substack{k=1 \\
k \neq l_{0}}}^{m} \mathcal{C}(m+1, k) L\left(\nu \hat{\vartheta}^{2}(m+1, k), \hat{a}_{m+1}\right)\right. \\
& \left.\left.+\mathcal{C}\left(l_{0}, m+1\right) L\left(\nu \hat{\vartheta}^{2}\left(l_{0}, m+1\right), \hat{a}_{l_{0}}\right)+\mathcal{C}\left(m+1, l_{0}\right) L\left(\nu \hat{\vartheta}\left(m+1, l_{0}\right), \hat{a}_{m+1}\right)\right\}\left(1-e^{-x_{i}}\right) \mid\left\{\hat{p}, \hat{f}, \hat{c}, \hat{c}^{\prime}\right\}_{m+1}\right) .
\end{aligned}
$$

Now, as long as neither $l$ nor $k$ equals $m+1$, the angle variable $\hat{\vartheta}(l, k)$ is very close to the corresponding angle variable $\vartheta(l, k)$ in the state $\left.\mid\left\{p, f, c, c^{\prime}\right\}_{m}\right)$ before the first splitting. The angle variable $\hat{\vartheta}(m+1, k)$ for $k \neq l_{0}$ is very close to $\vartheta\left(l_{0}, k\right)$ in the state before the first splitting, since partons $l_{0}$ and $m+1$ are nearly collinear in the integration region that can lead to a $\log (\nu)$ factor in the first splitting. Thus we regard these angles as fixed when calculating $\left.\mathcal{S}_{\mathcal{Y}}^{k+1]}\left(x_{0} Q^{2} / \nu ; \nu\right) \mid\left\{p, f, c, c^{\prime}\right\}_{m}\right)$. On the other hand, $\hat{\vartheta}\left(l_{0}, m+1\right)$ is the angle variable for the first splitting and is thus an integration variable in this calculation. Integrating over this variable can produce a $\log (\nu)$ factor. Thus we treat $\hat{\vartheta}\left(l_{0}, m+1\right)$ as potentially small in Eq. (A13), but we treat the other angle variables as being finite. For the purpose of finding $\log (\nu)$ factors, we simply replace these finite angle variables by 1 . These substitutions give us

$$
\begin{aligned}
\left.\mid A_{i}\right) \approx & -\left\{\sum_{\substack{l=1 \\
l \neq l_{0}}}^{m} \sum_{\substack{k=1 \\
k \neq l}}^{m+1} \mathcal{C}(l, k) L\left(\nu, \hat{a}_{l}\right)+\sum_{\substack{k=1 \\
k \neq l_{0}}}^{m} \mathcal{C}\left(l_{0}, k\right) L\left(\nu, \hat{a}_{l_{0}}\right)\right. \\
& +\sum_{\substack{k=1 \\
k \neq l_{0}}}^{m} \mathcal{C}(m+1, k) L\left(\nu, \hat{a}_{m+1}\right) \\
& +\mathcal{C}\left(l_{0}, m+1\right) L\left(\nu \hat{\vartheta}\left(l_{0}, m+1\right), \hat{a}_{l_{0}}\right) \\
& \left.+\mathcal{C}\left(l_{0}, m+1\right) L\left(\nu \hat{\vartheta}\left(l_{0}, m+1\right), \hat{a}_{m+1}\right)\right\} \\
& \left.\times\left(1-e^{-x_{i}}\right) \mid\left\{\hat{p}, \hat{f}, \hat{c}, \hat{c}^{\prime}\right\}_{m+1}\right) .
\end{aligned}
$$

In two of the terms in Eq. (A14), the parameter $\hat{a}_{m+1}$ appears. This parameter is large when the momentum fraction $1-z_{0}$ of parton $m+1$ in the first splitting is small:

$$
\hat{a}_{m+1} \approx \frac{a_{l_{0}}}{1-z_{0}} .
$$

We also note that the angle variable $\hat{\vartheta}\left(l_{0}, m+1\right)$ is proportional to $1 /\left(1-z_{0}\right)$ according to Eq. (113). We have

$$
\hat{\vartheta}\left(l_{0}, m+1\right) \approx \frac{a_{l_{0}}}{\nu\left(1-z_{0}\right)} .
$$

Combining these equations gives us

$$
\hat{a}_{m+1} \approx \nu \hat{\vartheta}\left(l_{0}, m+1\right) .
$$

With this replacement, the function $L$, Eq. (A11), in the last term in Eq. (A14) is approximately

$$
L\left(\nu \hat{\vartheta}\left(l_{0}, m+1\right), \hat{a}_{m+1}\right) \approx L\left(\hat{a}_{m+1}, \hat{a}_{m+1}\right)=0 .
$$

In the fourth term in Eq. (A14), we use this replacement to eliminate $\hat{\vartheta}\left(l_{0}, m+1\right)$ in favor of $\hat{a}_{m+1}$. With these substitutions, we have

$$
\begin{aligned}
\left.\mid A_{i}\right) \approx & -\left\{\sum_{\substack{l=1 \\
l \neq l_{0}}}^{m} \sum_{\substack{k=1 \\
k \neq l}}^{m+1} \mathcal{C}(l, k) L\left(\nu, \hat{a}_{l}\right)+\sum_{\substack{k=1 \\
k \neq l_{0}}}^{m} \mathcal{C}\left(l_{0}, k\right) L\left(\nu, \hat{a}_{l_{0}}\right)\right. \\
& +\sum_{\substack{k=1 \\
k \neq l_{0}}}^{m} \mathcal{C}(m+1, k) L\left(\nu, \hat{a}_{m+1}\right) \\
& \left.+\mathcal{C}\left(l_{0}, m+1\right) L\left(\hat{a}_{m+1}, \hat{a}_{l_{0}}\right)\right\} \\
& \left.\times\left(1-e^{-x_{i}}\right) \mid\left\{\hat{p}, \hat{f}, \hat{c}, \hat{c}^{\prime}\right\}_{m+1}\right) .
\end{aligned}
$$

Using the definition (A11) of $L(w, u)$, this function in the last term can be written as

$$
L\left(\hat{a}_{m+1}, \hat{a}_{l_{0}}\right)=-L\left(\nu, \hat{a}_{m+1}\right)+L\left(\nu, \hat{a}_{l_{0}}\right) .
$$

In the sum in the second term in Eq. (A19) we can add and subtract a contribution from $k=m+1$. After adding this contribution, the sum includes $k=m+1$, so that this sum can be combined with the sums in the first term. Then in the first term we can include $l=l_{0}$ in the sum over $l$. In the third term in Eq. (A19) we can add and subtract a contribution from $k=l_{0}$, so that after adding this contribution the sum includes $k=l_{0}$. With these changes, we have

$$
\begin{aligned}
\left.\mid A_{i}\right) \approx & -\left\{\sum_{l=1}^{m} \sum_{\substack{k=1 \\
k \neq l}}^{m+1} \mathcal{C}(l, k) L\left(\nu, \hat{a}_{l}\right)\right. \\
& +\sum_{k=1}^{m} \mathcal{C}(m+1, k) L\left(\nu, \hat{a}_{m+1}\right) \\
& \left.-2 \mathcal{C}\left(l_{0}, m+1\right) L\left(\nu, \hat{a}_{m+1}\right)\right\} \\
& \left.\times\left(1-e^{-x_{i}}\right) \mid\left\{\hat{p}, \hat{f}, \hat{c}, \hat{c}^{\prime}\right\}_{m+1}\right) .
\end{aligned}
$$

In the first term in Eq. (A21), we can use color conservation to write 


$$
\begin{aligned}
\sum_{\substack{k=1 \\
k \neq l}}^{m+1} \mathcal{C}(l, k) & =\sum_{\substack{k=1 \\
k \neq l}}^{m+1}\left[\boldsymbol{T}_{l} \cdot \boldsymbol{T}_{k} \otimes 1+1 \otimes \boldsymbol{T}_{l} \cdot \boldsymbol{T}_{k}\right] \\
& =-\left[\boldsymbol{T}_{l} \cdot \boldsymbol{T}_{l} \otimes 1+1 \otimes \boldsymbol{T}_{l} \cdot \boldsymbol{T}_{l}\right] \\
& =-2 C_{l}[1 \otimes 1],
\end{aligned}
$$

where $C_{l}=C_{\mathrm{A}}$ if parton $l$ is a gluon and $C_{l}=C_{\mathrm{F}}$ if parton $l$ is a quark or antiquark. The same applies to the second term:

$$
\sum_{k=1}^{m} \mathcal{C}(m+1, k)=-2 C_{\mathrm{A}}[1 \otimes 1]
$$

where we have used $C_{m+1}=C_{\mathrm{A}}$ since parton $m+1$ must be a gluon in order to give a leading $\log (\nu)$ contribution. These substitutions give us

$$
\begin{aligned}
\left.\mid A_{i}\right) \approx & \left\{\sum_{l=1}^{m} 2 C_{l}[1 \otimes 1] L\left(\nu, \hat{a}_{l}\right)\right. \\
& \left.+2\left[C_{\mathrm{A}}[1 \otimes 1]+\mathcal{C}\left(l_{0}, m+1\right)\right] L\left(\nu, \hat{a}_{m+1}\right)\right\} \\
& \left.\times\left(1-e^{-x_{i}}\right) \mid\left\{\hat{p}, \hat{f}, \hat{c}, \hat{c}^{\prime}\right\}_{m+1}\right) .
\end{aligned}
$$

Consider now the term in Eq. (A24) that contains a color operator $\mathcal{C}\left(l_{0}, m+1\right)$, defined in Eq. (A10). We apply this operator after the color operator for the initial splitting, $\mathcal{C}_{0}\left(l_{0}, k_{0}\right)$, defined in Eq. (A7). This gives us an operator with four terms,

$$
\begin{aligned}
\mathcal{C}_{i}= & \left(\boldsymbol{T}_{l_{0}} \cdot \boldsymbol{T}_{m+1}\right) T_{l_{0}} \otimes T_{k_{0}}^{\dagger}+T_{l_{0}} \otimes T_{k_{0}}^{\dagger}\left(\boldsymbol{T}_{l_{0}} \cdot \boldsymbol{T}_{m+1}\right) \\
& +\left(\boldsymbol{T}_{l_{0}} \cdot \boldsymbol{T}_{m+1}\right) T_{k_{0}} \otimes T_{l_{0}}^{\dagger}+T_{k_{0}} \otimes T_{l_{0}}^{\dagger}\left(\boldsymbol{T}_{l_{0}} \cdot \boldsymbol{T}_{m+1}\right) .
\end{aligned}
$$

There can be several factors of $\left[\mathcal{S}_{l}^{[1,0]}\left(x Q^{2} / \nu\right)\right]_{\mathbb{P}}$ in Eq. (A5) and in some of those factors we can select the $\mathcal{C}\left(l_{0}, m+1\right)$ term in Eq. (A24). Finally, there is a $[\cdots]_{\mathbb{P}}$ operation. This gives us a sum of color operators of the form

$$
\begin{aligned}
{[\mathcal{C}]_{\mathbb{P}}=} & {\left[\left(\boldsymbol{T}_{l_{0}} \cdot \boldsymbol{T}_{m+1}\right)^{A} T_{l_{0}} \otimes T_{k_{0}}^{\dagger}\left(\boldsymbol{T}_{l_{0}} \cdot \boldsymbol{T}_{m+1}\right)^{B}\right.} \\
& \left.+\left(\boldsymbol{T}_{l_{0}} \cdot \boldsymbol{T}_{m+1}\right)^{A} T_{k_{0}} \otimes T_{l_{0}}^{\dagger}\left(\boldsymbol{T}_{l_{0}} \cdot \boldsymbol{T}_{m+1}\right)^{B}\right]_{\mathbb{P}} .
\end{aligned}
$$

Using Eq. (158), this becomes

$$
\begin{aligned}
{[\mathcal{C}]_{\mathbb{P}}=} & {\left[\left(\boldsymbol{T}_{l_{0}} \cdot \boldsymbol{T}_{m+1}\right)^{A+B} T_{l_{0}} \otimes T_{k_{0}}^{\dagger}\right.} \\
& \left.+T_{k_{0}} \otimes T_{l_{0}}^{\dagger}\left(\boldsymbol{T}_{l_{0}} \cdot \boldsymbol{T}_{m+1}\right)^{A+B}\right]_{\mathbb{P}} .
\end{aligned}
$$

Now consider the color operator $\boldsymbol{T}_{l_{0}} \cdot \boldsymbol{T}_{m+1} T_{l_{0}}^{a}$. In diagrams, parton $l_{0}$ emits a gluon with label $m+1$, leaving parton $l_{0}$ in a new color state. Then a gluon is exchanged between partons $l_{0}$ and $m+1$. This gives us a color triangle diagram,

$$
\boldsymbol{T}_{l_{0}} \cdot \boldsymbol{T}_{m+1} T_{l_{0}}^{a}=\mathrm{i} f_{a b c} T_{l_{0}}^{b} T_{l_{0}}^{c}
$$

Then we can use

$\mathrm{i} f_{a b c} T_{l_{0}}^{b} T_{l_{0}}^{c}=\frac{1}{2} \mathrm{i} f_{a b c}\left[T_{l_{0}}^{b}, T_{l_{0}}^{c}\right]=\frac{1}{2} \mathrm{i} f_{a b c} \mathrm{i} f_{b c d} T_{l_{0}}^{d}=-\frac{C_{\mathrm{A}}}{2} T_{l_{0}}^{a}$.

Thus

$$
\boldsymbol{T}_{l_{0}} \cdot \boldsymbol{T}_{m+1} T_{l_{0}}^{a}=-\frac{C_{\mathrm{A}}}{2} T_{l_{0}}^{a} .
$$

This gives us

$$
\left(\boldsymbol{T}_{l_{0}} \cdot \boldsymbol{T}_{m+1}\right)^{A+B} T_{l_{0}} \otimes T_{k_{0}}^{\dagger}=\left[-\frac{C_{\mathrm{A}}}{2}\right]^{A+B} T_{l_{0}} \otimes T_{k_{0}}^{\dagger} .
$$

The second term in Eq. (A27) gives the same result, so that the net color operator defined in Eq. (A26) is

$$
[\mathcal{C}]_{\mathbb{P}}=\left[-\frac{C_{\mathrm{A}}}{2}\right]^{A+B}\left[T_{l_{0}} \otimes T_{k_{0}}^{\dagger}+T_{k_{0}} \otimes T_{l_{0}}^{\dagger}\right]_{\mathbb{P}} .
$$

We conclude that when $\mathcal{C}\left(l_{0}, m+1\right)$ in Eq. (A24) is part of $\mathcal{S}_{\mathcal{Y}}^{[k+1]}\left(x_{0} Q^{2} / \nu ; \nu\right)$ in Eq. (A5), we get the same result for $\mathcal{S}_{\mathcal{Y}}^{[k+1]}\left(x_{0} Q^{2} / \nu ; \nu\right)$ by making the replacement

$$
\mathcal{C}\left(l_{0}, m+1\right) \rightarrow-C_{\mathrm{A}}[1 \otimes 1]
$$

There is a factor 2 for each $C_{\mathrm{A}}$ here because there are two $T_{l_{0}} \otimes T_{k_{0}}^{\dagger}$ terms and two $T_{k_{0}} \otimes T_{l_{0}}^{\dagger}$ terms in Eq. (A25).

With this replacement, the terms in Eq. (A24) proportional to $L\left(\nu, \hat{a}_{m+1}\right)$ cancel. Thus we get the same result for $\mathcal{S}_{\mathcal{Y}}^{[k+1]}\left(x_{0} Q^{2} / \nu ; \nu\right)$ by making the replacement

$$
\left.\left.\mid A_{i}\right) \rightarrow \mid A_{i}^{\mathrm{eff}}\right),
$$

where

$$
\left.\left.\mid A_{i}^{\mathrm{eff}}\right) \approx \sum_{l=1}^{m} 2 C_{l}[1 \otimes 1] L\left(\nu, \hat{a}_{l}\right)\left(1-e^{-x_{i}}\right) \mid\left\{\hat{p}, \hat{f}, \hat{c}, \hat{c}^{\prime}\right\}_{m+1}\right) .
$$

Note that $\left.\mid A_{i}^{\text {eff }}\right)$ is a number, which we may call $\lambda_{i}$, times the starting state vector,

$$
\left.\left.\mid A_{i}^{\mathrm{eff}}\right)=\lambda_{i} \mid\left\{\hat{p}, \hat{f}, \hat{c}, \hat{c}^{\prime}\right\}_{m+1}\right)
$$


Return now to Eq. (A5) for $\mathcal{S}_{\mathcal{Y}}^{[k+1]}\left(x_{0} Q^{2} / \nu ; \nu\right)$ applied to the starting state $\left.\mid\left\{p, f, c, c^{\prime}\right\}_{m}\right)$. In the last factor, we have dealt with the operator $\mathcal{S}_{l_{0}}^{[1,0]}\left(Q^{2} / \nu\right)$, which creates a new parton with label $m+1$. Now we turn to the remaining operator, $-\left[\mathcal{S}_{l_{0}}^{[1,0]}\left(Q^{2} / \nu\right)\right]_{\mathbb{P}}$. This operator, acting on the state $\left.\mid\left\{p, f, c, c^{\prime}\right\}_{m}\right)$, produces a linear combination of states with $m$ partons, $\left.\mid\left\{p, f, \hat{c}, \hat{c}^{\prime}\right\}_{m}\right)$. Here the momentum and flavors are the same as in the initial state, but the colors change. More precisely,

$$
\begin{aligned}
\sum_{l_{0}=1}^{m}[ & \left.\left.\mathcal{S}_{l_{0}}^{[1,0]}\left(Q^{2} / \nu\right)\right]_{\mathbb{P}} e^{-x_{0}} \mid\left\{p, f, c, c^{\prime}\right\}_{m}\right) \\
\approx & \left.-\sum_{l_{0}=1}^{m} \sum_{\substack{k_{0}=1 \\
k_{0} \neq l_{0}}}^{m}\left[\mathcal{C}\left(l_{0}, k_{0}\right)\right]_{\mathbb{P}} \mid\left\{p, f, c, c^{\prime}\right\}_{m}\right) \\
& \times \int \frac{d \phi_{0}}{2 \pi} \int \frac{d z_{0}}{1-z_{0}} \frac{\alpha_{s}\left(\lambda_{\mathrm{R}}\left(1-z_{0}\right) Q^{2} /\left(\nu a_{l_{0}}\right)\right)}{2 \pi} \\
& \times \Theta\left(\frac{a_{l_{0}}}{\nu \vartheta\left(l_{0}, k_{0}\right)}<1-z_{0}<1\right) e^{-x_{0}} .
\end{aligned}
$$

Let us consider what the one of the operators, $\left[\mathcal{S}_{l_{i}}^{[1,0]}\left(Q^{2} / \nu\right)\right]_{\mathbb{P}}$, in Eq. (A5) does to this state. We consider the quantity

$\left.\left.\mid B_{i}\right)=\sum_{l_{i}=1}^{m+1}\left[\mathcal{S}_{l_{i}}^{[1,0]}\left(Q^{2} / \nu\right)\right]_{\mathbb{P}}\left(1-e^{-x_{i}}\right) \mid\left\{p, f, \hat{c}, \hat{c}^{\prime}\right\}_{m}\right)$.

With an analysis similar to but simpler than our previous analysis, we obtain

$$
\left.\left.\mid B_{i}\right) \approx \sum_{l=1}^{m} 2 C_{l}[1 \otimes 1] L\left(\nu, \hat{a}_{l}\right)\left(1-e^{-x_{i}}\right) \mid\left\{p, f, \hat{c}, \hat{c}^{\prime}\right\}_{m}\right) .
$$

This gives us

$$
\left.\left.\mid B_{i}\right)=\lambda_{i} \mid\left\{p, f, \hat{c}, \hat{c}^{\prime}\right\}_{m}\right),
$$

where the eigenvalue $\lambda_{i}$ is exactly the $\lambda_{i}$ in Eq. (A36).

We can substitute Eqs. (A40) and (A36) into Eq. (A5) to obtain

$$
\begin{aligned}
\mathcal{S}_{\mathcal{Y}}^{[k+1]} & \left.\left(x_{0} Q^{2} / \nu ; \nu\right) \mid\left\{p, f, c, c^{\prime}\right\}_{m}\right) \\
= & (-1)^{n} \int_{0}^{x_{0}} \frac{d x_{1}}{x_{1}} \int_{0}^{x_{1}} \frac{d x_{2}}{x_{2}} \cdots \int_{0}^{x_{k-1}} \frac{d x_{k}}{x_{k}} \lambda_{k} \cdots \lambda_{2} \lambda_{1} \\
& \left.\times \sum_{l_{0}}\left[\left[\mathcal{S}_{l_{0}}^{[1,0]}\left(Q^{2} / \nu\right)\right]_{1-\mathbb{P}}\right]_{\mathbb{P}} e^{-x_{0}} \mid\left\{p, f, c, c^{\prime}\right\}_{m}\right) \\
& +\mathrm{NNLL} .
\end{aligned}
$$

However

$$
\begin{aligned}
{\left[\left[\mathcal{S}_{l_{0}}^{[1,0]}\left(Q^{2} / \nu\right)\right]_{1-\mathbb{P}}\right]_{\mathbb{P}} } & =\left[\mathcal{S}_{l_{0}}^{[1,0]}\left(Q^{2} / \nu\right)-\left[\mathcal{S}_{l_{0}}^{[1,0]}\left(Q^{2} / \nu\right)\right]_{\mathbb{P}}\right]_{\mathbb{P}} \\
& =\left[\mathcal{S}_{l_{0}}^{[1,0]}\left(Q^{2} / \nu\right)\right]_{\mathbb{P}}-\left[\mathcal{S}_{l_{0}}^{[1,0]}\left(Q^{2} / \nu\right)\right]_{\mathbb{P}} \\
& =0 .
\end{aligned}
$$

Thus the NLL contributions to $\mathcal{S}_{\mathcal{Y}}^{k+1]}\left(x_{0} Q^{2} / \nu ; \nu\right)$ vanish:

$$
\left.\mathcal{S}_{\mathcal{Y}}^{[k+1]}\left(x_{0} Q^{2} / \nu ; \nu\right) \mid\left\{p, f, c, c^{\prime}\right\}_{m}\right)=\mathrm{NNLL}
$$

\section{APPENDIX B: CANCELLATION WITH $k_{\mathrm{T}}$ ORDERING}

In this appendix, we explore the cancellation of large $\log (\nu)$ factors in $\mathcal{I}_{2}^{[2]}(\nu)$ with $k_{\mathrm{T}}$ ordering. We can write $\left(1\left|\mathcal{I}_{2}^{[2]}(\nu)\right|\left\{\tilde{p}, \tilde{f}, \tilde{c}, \tilde{c}^{\prime}\right\}_{2}\right)$ in the form

$$
\begin{aligned}
\left(1\left|\mathcal{I}^{[2]}(\nu)\right|\left\{\tilde{p}, \tilde{f}, \tilde{c}, \tilde{c}^{\prime}\right\}_{2}\right) \\
=\int_{0}^{Q^{2}} \frac{d \tilde{k}_{T}}{\tilde{k}_{T}} \int d \tilde{\eta} \int \frac{d \tilde{\phi}}{2 \pi} \int_{0}^{Q^{2}} \frac{d k_{T}}{k_{T}} \int d \eta \int \frac{d \phi}{2 \pi} \\
\quad \times \Theta\left(k_{\mathrm{T}}<\tilde{k}_{\mathrm{T}}\right)\left(1-e^{\nu(\hat{\tau}-\tau)}\right) e^{-\nu \tau}\left(1 \mid \mathcal{S}^{[1,0]}\left(k_{\mathrm{T}}, \eta, \phi\right)\right. \\
\quad \times\left\{\mathcal{S}^{[1,0]}\left(\tilde{k}_{\mathrm{T}}, \tilde{\eta}, \tilde{\phi}\right)-\left[\mathcal{S}^{[1,0]}\left(\tilde{k}_{\mathrm{T}}, \tilde{\eta}, \tilde{\phi}\right)\right]_{\mathbb{P}}\right\} \\
\left.\quad \times \mid\left\{\tilde{p}, \tilde{f}, \tilde{c}, \tilde{c}^{\prime}\right\}_{2}\right) .
\end{aligned}
$$

We begin with a $q \bar{q}$ state with parton momenta $\tilde{p}_{1}$ and $\tilde{p}_{2}$ aligned along the + and $-z$ axis, respectively. Then one of these two partons splits, producing parton 3 . We suppose that it is parton 1 that splits. After the splitting, we have partons with momenta $p_{1}, p_{2}$, and $p_{3}$. The value of $1-T$ in this state is $\tau$ and we suppose that $\tau \ll 1$. Then there is a second splitting, producing partons with momenta $\hat{p}_{1}, \hat{p}_{2}$, $\hat{p}_{3}$, and $\hat{p}_{4}$ with a thrust variable $\hat{\tau} \ll 1$. We consider either the splitting of parton 3 with parton 2 as the dipole partner or the splitting of parton 2 with parton 3 as dipole partner. Other splitting possibilities are not as important and we omit consideration of them here. We limit our consideration to the leading color approximation.

We begin with the first splitting, which we describe with splitting variables $\tilde{k}_{\mathrm{T}}, \tilde{\eta}, \tilde{\phi}$ that relate $p_{3}$ to $\tilde{p}_{1}$ and $\tilde{p}_{2}$ :

$$
p_{3}=e^{\tilde{\eta}} \frac{\tilde{k}_{\mathrm{T}}}{|Q|} \tilde{p}_{1}+e^{-\tilde{\eta}} \frac{\tilde{k}_{\mathrm{T}}}{|Q|} \tilde{p}_{2}+\tilde{k}_{\perp}
$$

Here $|Q|=\left[Q^{2}\right]^{1 / 2}=\left[2 \tilde{p}_{1} \cdot \tilde{p}_{2}\right]^{1 / 2}$ and $\tilde{k}_{\perp}$ is a vector that is orthogonal to $\tilde{p}_{1}$ and $\tilde{p}_{2}$ :

$$
\tilde{k}_{\perp} \cdot \tilde{p}_{1}=\tilde{k}_{\perp} \cdot \tilde{p}_{2}=0 .
$$

We have defined the scalar $\tilde{k}_{\mathrm{T}}$ by 


$$
\tilde{k}_{\mathrm{T}}=\left[-\tilde{k}_{\perp}^{2}\right]^{1 / 2}
$$

This definition gives $p_{3}^{2}=0$. The variable $\tilde{\eta}$ is the rapidity of $p_{3}$. We need one more splitting variable, the azimuthal angle $\tilde{\phi}$ of $\tilde{k}_{\perp}$.

For emission from parton 1 , the splitting function is small for $\tilde{\eta}<0$. There is a maximum value of $\tilde{\eta}$ for fixed $\tilde{k}_{\mathrm{T}}$, set by the condition for a maximally collinear emission

$$
e^{\tilde{\eta}} \frac{\tilde{k}_{\mathrm{T}}}{|Q|}=1
$$

When $\tilde{\eta}$ is close to this upper bound, the splitting function tends to zero. Thus we integrate over the splitting variables with measure $d \tilde{\eta} d \log \left(\tilde{k}_{\mathrm{T}} /|Q|\right)$ over the range $0 \lesssim \tilde{\eta} \lesssim-\log \left(\tilde{k}_{\mathrm{T}} /|Q|\right)$. In this range, as long as $\tilde{\eta}$ is not near either endpoint, the splitting function is approximately constant. For small $\tilde{k}_{\mathrm{T}}$, this is a large range. The integration gives us a large logarithm, which comes from integrating over the interior of the range, omitting the regions near the endpoints:

$$
0 \ll \tilde{\eta} \ll-\log \left(\tilde{k}_{\mathrm{T}} /|Q|\right) .
$$

We will assume that $\tilde{\eta}$ lies in this range in the analysis that follows.

For an emission from parton 1, we define the momentum of parton 1 after the emission to be

$$
p_{1}=\left[1-e^{\tilde{\eta}} \frac{\tilde{k}_{\mathrm{T}}}{|Q|}\right] \tilde{p}_{1}+\frac{\tilde{k}_{\mathrm{T}}^{2} /|Q|^{2}}{1-e^{\tilde{\eta}} \tilde{k}_{\mathrm{T}} /|Q|} \tilde{p}_{2}-\tilde{k}_{\perp} .
$$

With this definition, $p_{1}^{2}=0$ and $p_{1}-\tilde{p}_{1}+p_{3}$ lies entirely in the direction of $\tilde{p}_{2}$ :

$$
p_{1}-\tilde{p}_{1}+p_{3}=\frac{e^{-\tilde{\eta}} \tilde{k}_{\mathrm{T}} /|Q|}{1-e^{\tilde{\eta}} \tilde{k}_{\mathrm{T}} /|Q|} \tilde{p}_{2} .
$$

Finally, we need to define the momentum $p_{2}$ of parton 2 after the splitting so that momentum is conserved: $p_{1}+p_{2}+p_{3}=\tilde{p}_{1}+\tilde{p}_{2}$. Using Eq. (B8) we obtain $p_{2}$ by applying a small boost in the $z$ direction to $\tilde{p}_{2}$ :

$$
p_{2}=\left[1-\frac{e^{-\tilde{\eta}} \tilde{k}_{\mathrm{T}} /|Q|}{1-e^{\tilde{\eta}} \tilde{k}_{\mathrm{T}} /|Q|}\right] \tilde{p}_{2} .
$$

This is the exact relation. In the integration range (B6), this relation becomes

$$
\tilde{p}_{2}-p_{2} \approx e^{-\tilde{\eta}} \frac{\tilde{k}_{\mathrm{T}}}{|Q|} \tilde{p}_{2}
$$

We use Eqs. (123) and (124) to calculate the thrust for the state after the first splitting:

$$
\begin{aligned}
\tau & =\frac{1}{Q^{-}}\left(p_{1}^{-}+p_{3}^{-}+p_{2}^{+}\right) \\
& =\frac{1}{Q^{-}}\left(\tilde{p}_{2}^{-}-p_{2}^{-}+\tilde{p}_{1}^{-}+p_{2}^{+}\right) .
\end{aligned}
$$

We can use $\tilde{p}_{1}^{-}=p_{2}^{+}=0$. Then we can use $\tilde{p}_{2}^{-}=Q^{-}$and Eq. (B10) for $\tilde{p}_{2}^{-}-p_{2}^{-}$. This gives $\tau \approx e^{-\tilde{\eta}} \tilde{k}_{\mathrm{T}} /|Q|$ or

$$
\nu \tau \approx \nu e^{-\tilde{\eta}} \frac{\tilde{k}_{\mathrm{T}}}{|Q|} .
$$

This relation is significant because this emission is accompanied by a measurement function $\exp (-\nu \tau)$. The measurement function is approximately 1 for $\nu \tau \ll 1$ but approximately zero for $1 \ll \nu \tau$. Thus we effectively integrate over the range

$$
\nu \tau<1
$$

In the analysis that follows, we will need a relation between $2 p_{3} \cdot Q$ and the values of $\tilde{\eta}$ and $\tilde{k}_{\mathrm{T}}$ for the splitting. We can use Eq. (B2) with $\eta \gg 0$ together with $2 \tilde{p}_{1} \cdot Q=$ $Q^{2}$ to give

$$
\frac{2 p_{3} \cdot Q}{Q^{2}}=e^{\tilde{\eta}} \frac{\tilde{k}_{\mathrm{T}}}{|Q|}
$$

We now turn to the second splitting. We will describe the splitting using variables and a momentum mapping that are slightly different from what is used in DEDUCTOR with $k_{\mathrm{T}}$ ordering. In fact, we will use a local momentum mapping. However, in the kinematic limit of interest, the description used here reduces to the description used in DEDUCTOR. The splitting kinematics are illustrated in Fig. 15. We describe the second splitting with splitting variables $k_{\mathrm{T}}, \eta$, $\phi$ that relate $\hat{p}_{4}$ to $p_{2}$ and $p_{3}$ :

$$
\hat{p}_{4}=A_{32} e^{\eta} \frac{k_{\mathrm{T}}}{|Q|} p_{3}+A_{23} e^{-\eta} \frac{k_{\mathrm{T}}}{|Q|} p_{2}+k_{\perp},
$$

where

$$
\begin{aligned}
& A_{32}=\left[\frac{Q^{2}}{2 p_{2} \cdot p_{3}} \frac{p_{2} \cdot Q}{p_{3} \cdot Q}\right]^{1 / 2}, \\
& A_{23}=\left[\frac{Q^{2}}{2 p_{2} \cdot p_{3}} \frac{p_{3} \cdot Q}{p_{2} \cdot Q}\right]^{1 / 2} .
\end{aligned}
$$

Here $k_{\perp}$ is a vector that is orthogonal to $p_{2}$ and $p_{3}$ :

$$
k_{\perp} \cdot p_{3}=k_{\perp} \cdot p_{2}=0 .
$$

As for the first splitting, we have defined the scalar $k_{\mathrm{T}}=\left[-k_{\perp}^{2}\right]^{1 / 2}$. This definition gives $\hat{p}_{4}^{2}=0$. The variable $\eta$ describes the rapidity of $\hat{p}_{4}$ with respect to the emitting 
dipole, with a constant $\log \left(p_{2} \cdot Q / p_{3} \cdot Q\right) / 2$ added [16]. We need one more splitting variable, the azimuthal angle $\phi$ of $k_{\perp}$ in the dipole c.m. frame.

There is a limit to how large $\eta$ can be: $\eta_{\min }<\eta<\eta_{\max }$. The limits are fixed by the requirements that the components of $\hat{p}_{4}$ along $p_{3}$ and $p_{2}$ cannot be larger than 1:

$$
\begin{aligned}
& \eta_{\max }=-\log \left(\frac{k_{\mathrm{T}}}{|Q|}\right)-\log \left(A_{32}\right), \\
& \eta_{\text {min }}=\log \left(\frac{k_{\mathrm{T}}}{|Q|}\right)+\log \left(A_{23}\right) .
\end{aligned}
$$

The lines $\eta=\eta_{\max }$ and $\eta=\eta_{\min }$ are indicated in Fig. 15 as the lines labeled collinear. This is a large integration range. We will assume in what follows that $\eta$ is not near to the endpoints of the integration range:

$$
\eta_{\min } \ll \eta \ll \eta_{\max }
$$

For emission from parton 3, we let the momentum of parton 3 after the emission be

$$
\begin{aligned}
\hat{p}_{3} \approx & {\left[1-A_{32} e^{\eta} \frac{k_{\mathrm{T}}}{|Q|}\right] p_{3} } \\
& +\frac{k_{\mathrm{T}}^{2}}{Q^{2}} \frac{Q^{2}}{2 p_{3} \cdot p_{2}}\left[1-A_{32} e^{\eta} \frac{k_{\mathrm{T}}}{|Q|}\right]^{-1} p_{2}-k_{\perp} .
\end{aligned}
$$

With this definition, $\hat{p}_{3}^{2}=0$ and $\hat{p}_{3}-p_{3}+\hat{p}_{4}$ lies entirely in the direction of $p_{2}$. Then we can maintain momentum conservation, $\hat{p}_{1}+\hat{p}_{2}+\hat{p}_{3}+\hat{p}_{4}=p_{1}+p_{2}+p_{3}$ by setting $\hat{p}_{1}=p_{1}$ and obtaining $\hat{p}_{2}$ by performing a small boost on $p_{2}$ :

$$
\hat{p}_{2}=e^{-\omega} p_{2}
$$

With a few algebraic steps, we find

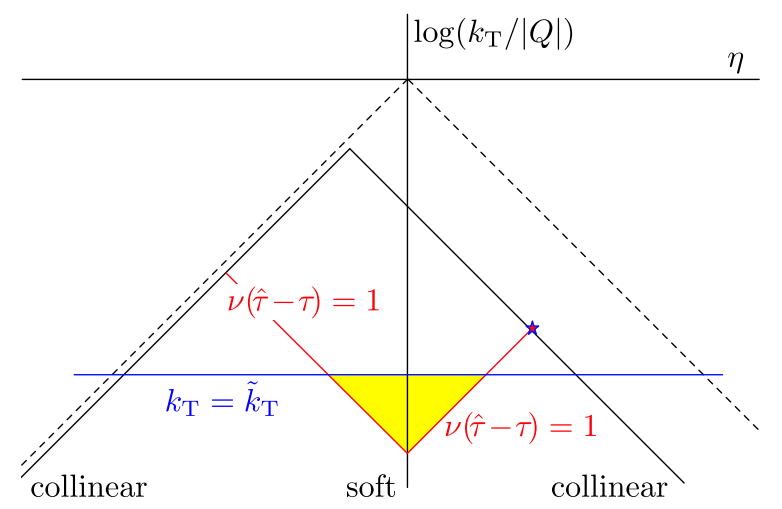

FIG. 15. Integration regions for second splitting.

$$
e^{-\omega}=1-A_{23} e^{-\eta} \frac{k_{\mathrm{T}}}{|Q|}\left[1-A_{32} e^{\eta} \frac{k_{\mathrm{T}}}{|Q|}\right]^{-1} .
$$

These definitions have been exact for the kinematic variables and momentum mapping chosen. We can now make some approximations. Given our kinematic conditions (B6) for the first emission, the momentum $p_{3}$ has large rapidity. That is, it makes a small angle with the $z$ axis. The transverse momentum vector defined in Eq. (B15) is orthogonal to $p_{3}$ and $p_{2}$ whereas the transverse momentum vector in DEDUCTOR is orthogonal to $p_{3}$ and $Q$. However, since $p_{3}$ makes a small angle with the $z$ axis, this is almost the same thing. In DEDUCTOR, momentum is conserved by applying a boost in the plane of $p_{3}$ and $Q$. Since $p_{3}$ makes a small angle with the $z$ axis, this boost is almost exactly along the $z$ axis. The boost is applied to both $p_{2}$ and $p_{1}$, but this difference has only a tiny effect on the resulting thrust. Thus in the limit considered, the DEDUCTOR kinematics and the kinematics used here are equivalent.

We now examine the change in thrust produced by the emission of parton 4 from parton 3 . We assume that $p_{4}$ is in the right thrust hemisphere. This is always the case when $\eta \gg 0$. There is a region near $\eta \approx 0$ in which this assumption fails. With the kinematics that we are using, the thrust axis is along $-\vec{p}_{2}$. That is, it is the $z$ axis. Then we have

$$
\begin{aligned}
\hat{\tau}-\tau & =\frac{1}{Q^{-}}\left[\hat{p}_{4}^{-}+\hat{p}_{3}^{-}-p_{3}^{-}+\hat{p}_{2}^{+}-p_{2}^{+}\right] \\
& =\frac{1}{Q^{-}}\left[p_{2}^{-}-\hat{p}_{2}^{-}+\hat{p}_{2}^{+}-p_{2}^{+}\right] .
\end{aligned}
$$

We have $p_{2}^{+}=0, \hat{p}_{2}=e^{-\omega} p_{2}$ from Eq. (B21), and $p_{2}^{-} / Q^{-}=2 p_{2} \cdot Q / Q^{2}$. This gives us

$$
\hat{\tau}-\tau=\frac{2 p_{2} \cdot Q}{Q^{2}}\left[1-e^{-\omega}\right] .
$$

Now the condition $\eta \ll \eta_{\max }$ that we assume implies that $A_{32} e^{\eta} k_{\mathrm{T}} /|Q| \ll 1$. Thus in Eq. (B22), we can replace the factor $1-A_{32} e^{\eta} k_{\mathrm{T}} /|Q|$ in $e^{-\omega}$ by just 1 . Then

$$
\hat{\tau}-\tau=\frac{2 p_{2} \cdot Q}{Q^{2}} A_{23} e^{-\eta} \frac{k_{\mathrm{T}}}{|Q|} .
$$

Since $p_{3}$ makes a small angle with the $z$ axis, we obtain the approximations

$$
\begin{aligned}
2 p_{2} \cdot p_{3} & \approx \frac{2 p_{2} \cdot Q 2 p_{3} \cdot Q}{Q^{2}}, \\
A_{32} & \approx \frac{Q^{2}}{2 p_{3} \cdot Q}, \\
A_{23} & \approx \frac{Q^{2}}{2 p_{2} \cdot Q} .
\end{aligned}
$$


With these approximations, we have

$$
\nu(\hat{\tau}-\tau) \approx \nu e^{-\eta} \frac{k_{\mathrm{T}}}{|Q|} .
$$

With the same approximations, we obtain for the change in thrust produced by an emission from parton 2 with the dipole partner being parton 3,

$$
\nu(\hat{\tau}-\tau) \approx \nu e^{\eta} \frac{k_{\mathrm{T}}}{|Q|} .
$$

Again, this is for $|\eta| \gg 0$. For the soft emission region near $\eta=0$, there is the possibility that $p_{4}$ is in the opposite thrust hemisphere from the parton that emitted it, so that the thrust calculation changes.

These relations are significant because the second emission is accompanied by a measurement function $1-\exp (-\nu(\hat{\tau}-\tau))$. The measurement function is approximately 1 for $1 \ll \nu(\hat{\tau}-\tau)$ but approximately zero for $\nu(\hat{\tau}-\tau) \ll 1$. Thus we effectively integrate over the range

$$
\nu(\hat{\tau}-\tau)>1 .
$$

The boundary of this integration region is indicated in Fig. 15 as straight lines with the labels $\nu(\hat{\tau}-\tau)=1$.

There is one more restriction on the integration range for the second splitting. We are analyzing a $k_{\mathrm{T}}$ ordered shower, so

$$
k_{\mathrm{T}}<\tilde{k}_{\mathrm{T}}
$$

The line $k_{\mathrm{T}}=\tilde{k}_{\mathrm{T}}$ is indicated in Fig. 15 .

To analyze Eq. (B30), we will need to know the value $k_{\mathrm{T}, \star}$ of $k_{\mathrm{T}}$ at the point labeled with a star in Fig. 15. We first note that the line for $\eta>0$ labeled collinear in Fig. 15 is given by $\eta=\eta_{\max }$ in Eq. (B18), $e^{\eta} k_{\mathrm{T}} /|Q|=1 / A_{32}$. We can use Eqs. (B26) and (B14) for $A_{32}$, giving

$$
e^{\eta} \frac{k_{\mathrm{T}}}{|Q|} \approx e^{\tilde{\eta}} \frac{\tilde{k}_{\mathrm{T}}}{|Q|}, \quad \text { collinear. }
$$

Then using Eq. (B12) to eliminate $\tilde{\eta}$ and Eq. (B27) to eliminate $\eta$ we have

$$
\frac{k_{\mathrm{T}}^{2}}{Q^{2}} \approx \frac{\nu(\hat{\tau}-\tau)}{\nu \tau} \frac{\tilde{k}_{\mathrm{T}}^{2}}{Q^{2}}, \quad \text { collinear. }
$$

The point labeled with a star in Fig. 15 is the intersection of the collinear line and the line $\nu(\hat{\tau}-\tau)=1$. Thus,

$$
\frac{k_{\mathrm{T}, \star}^{2}}{Q^{2}} \approx \frac{1}{\nu \tau} \frac{\tilde{k}_{\mathrm{T}}^{2}}{Q^{2}}
$$

Since in the dominant integration region $\nu \tau<1$, we conclude that $k_{\mathrm{T}, \star}>\tilde{k}_{\mathrm{T}}$. Thus the line $k_{\mathrm{T}}=\tilde{k}_{\mathrm{T}}$ lies below the point $\left(\eta_{\star}, k_{\mathrm{T}, \star}\right)$ in Fig. 15. This implies that the effective integration region for the second splitting is the region shaded in yellow in Fig. 15. Inside this region, the integrand is approximately 1 .

Now consider the case in which the first splitting is virtual. The corresponding contribution comes from the term $\left[\mathcal{S}^{[1,0]}\left(\tilde{k}_{\mathrm{T}}, \tilde{\eta}, \tilde{\phi}\right) e^{-\nu \tau}\right]_{\mathbb{P}}$ in the last line of Eq. (B1). We integrate over the splitting variables for the first splitting, including the measurement function $e^{-\nu \tau}$, but we start the second splitting from the $q \bar{q}$ state with just partons with momenta $\tilde{p}_{1}$ and $\tilde{p}_{2}$, but with the $k_{\mathrm{T}}$ ordering requirement $k_{\mathrm{T}}<\tilde{k}_{\mathrm{T}}$. Now the limits on $\eta$ in Fig. 15 , indicated by the lines labeled collinear, are expanded to the dotted lines in the figure. However, the effective integration region for the second splitting is the region shaded in yellow in Fig. 15. When we subtract the virtual contribution from the real contribution, we get zero within the approximations that we have used.

In Eq. (B33), we have equality, $\tilde{k}_{\mathrm{T}}=k_{\mathrm{T}, \star}$, when the value of $\tau$ for the first splitting is given by $\nu \tau=1$. The value of $\tilde{k}_{\mathrm{T}}$ in the first splitting can be less than $k_{\mathrm{T}, \star}$, but if $\tilde{k}_{\mathrm{T}}$ is too small then the integration region in Fig. 15 disappears. From Eq. (B27) at $\eta=0, \nu(\hat{\tau}-\tau)=1$ and $\tilde{k}_{\mathrm{T}}=k_{\mathrm{T}}$, we see that this limits $\tilde{k}_{\mathrm{T}}$ to

$$
\frac{\tilde{k}_{\mathrm{T}}}{|Q|}>\frac{1}{\nu} .
$$

Our analysis above has assumed that the first emission is at large rapidity, $\tilde{\eta} \gg 0$. What happens when $\tilde{\eta} \approx 0$ ? The approximations that we have used are not adequate in this situation, so it might seem that there is nothing that we can say. However, we can examine what happens when $\tilde{\eta}$ is large enough that the approximations are still valid, but $\tilde{\eta}$ becomes smaller and smaller. Start with Eq. (B31) for the collinear line in Fig. 15 and use Eq. (B12) to eliminate $\tilde{k}_{\mathrm{T}}$ and Eq. (B27) to eliminate $k_{\mathrm{T}}$, giving

$$
e^{2 \eta} \approx \frac{\nu \tau}{\nu(\hat{\tau}-\tau)} e^{2 \tilde{\eta}}, \quad \text { collinear. }
$$

The point labeled with a star in Fig. 15 is the intersection of the collinear line and the line $\nu(\hat{\tau}-\tau)=1$. Thus,

$$
e^{2 \eta_{\star}} \approx \nu \tau e^{2 \tilde{\eta}}
$$

In the effective integration range for the first splitting, we have $\nu \tau<1$. Thus

$$
\eta_{\star}<\tilde{\eta}
$$

This tells us that when the rapidity of the first splitting becomes small, $\tilde{\eta} \rightarrow 0$, we have $\eta_{\star} \rightarrow 0$. In this limit, the real-virtual cancellation in this region deteriorates, but this deterioration does not matter because the allowed 
integration region for the second splitting in Fig. 15 shrinks to zero.

The cancellation will fail on a certain surface in the integration region. On this surface, the splitting variables for the second emission are given by

$$
\left(k_{\mathrm{T}}, \eta\right) \approx\left(k_{\mathrm{T}, \star}, \eta_{\star}\right)
$$

In this region, the second emission is collinear rather than both soft and collinear, so that the emission probability does not match the constant that appears in the region in which the second emission is both soft and collinear. However in the virtual subtraction the second emission is both soft and collinear so that the emission probability is this constant. Thus the emission probabilities do not match between the real emission and the subtraction.

The surface of non-matching probabilities is specified as follows. If $k_{\mathrm{T}}=k_{\mathrm{T}, \star}$, then the line $k_{\mathrm{T}}=\tilde{k}_{\mathrm{T}}$ in Fig. 15 must pass through $\left(k_{\mathrm{T}, \star}, \eta_{\star}\right)$, so that $\tilde{k}_{\mathrm{T}}=k_{\mathrm{T}, \star}$. Then Eq. (B33) implies that the value of $\tau$ for the first emission is given by $\nu \tau=1$. Then Eq. (B12) gives

$$
\tilde{\eta} \approx \log (\nu)+\log \left(\frac{\tilde{k}_{\mathrm{T}}}{Q}\right)
$$

The transverse momentum for the first emission varies in the range

$$
-\log (\nu) \ll \log \left(\frac{\tilde{k}_{\mathrm{T}}}{Q}\right) \ll-\frac{1}{2} \log (\nu) .
$$

Here the lower limit is from Eq. (B34) and the upper limit is from Eqs. (B6), (B12), and (B13). For the second emission, $\left(k_{\mathrm{T}}, \eta\right) \approx\left(k_{\mathrm{T}, \star}, \eta_{\star}\right)$ :

$$
\begin{aligned}
\eta & \approx \tilde{\eta}, \\
\log \left(\frac{k_{\mathrm{T}}}{Q}\right) & \approx \log \left(\frac{\tilde{k}_{\mathrm{T}}}{Q}\right) .
\end{aligned}
$$

Thus the integration region inside which cancellation fails is one dimensional, so we are left with a contribution to $\mathcal{I}_{2}^{[2]}$ proportional to $\log ^{1}(\nu)$.
[1] G. Marchesini and B. R. Webber, Simulation of QCD jets including soft gluon interference, Nucl. Phys. B238, 1 (1984).

[2] G. Ingelman and D. E. Soper, Comparison of Monte Carlo and analytical calculations in QCD, Phys. Lett. 148B, 171 (1984).

[3] G. Marchesini, B. R. Webber, G. Abbiendi, I. G. Knowles, M. H. Seymour, and L. Stanco, HERWIG 5.1 - a Monte Carlo event generator for simulating hadron emission reactions with interfering gluons, Comput. Phys. Commun. 67, 465 (1992).

[4] M. Bahr et al., Herwig++ physics and manual, Eur. Phys. J. C 58, 639 (2008).

[5] S. C. Chao, D. E. Soper, and J. C. Collins, The order $\alpha_{s}^{2}$ energy-energy correlation function at small angles, Nucl. Phys. B214, 513 (1983).

[6] T. D. Gottschalk, A realistic model for e+ e- annihilation including parton Bremsstrahlung effects, Nucl. Phys. B214, 201 (1983).

[7] S. Catani, B. R. Webber, and G. Marchesini, QCD coherent branching and semiinclusive processes at large $\mathrm{x}$, Nucl. Phys. B349, 635 (1991).

[8] A. Banfi, G. Corcella, and M. Dasgupta, Angular ordering and parton showers for non-global QCD observables, J. High Energy Phys. 03 (2007) 050.

[9] Y.L. Dokshitzer and G. Marchesini, Monte Carlo and large angle gluon radiation, J. High Energy Phys. 03 (2009) 117.

[10] Z. Nagy and D. E. Soper, Final state dipole showers and the DGLAP equation, J. High Energy Phys. 05 (2009) 088.
[11] P. Z. Skands and S. Weinzierl, Some remarks on dipole showers and the DGLAP equation, Phys. Rev. D 79, 074021 (2009).

[12] Z. Nagy and D. E. Soper, On the transverse momentum in Z-boson production in a virtuality ordered parton shower, J. High Energy Phys. 03 (2010) 097.

[13] S. Höche, D. Reichelt, and F. Siegert, Momentum conservation and unitarity in parton showers and NLL resummation, J. High Energy Phys. 01 (2018) 118.

[14] M. Dasgupta, F. A. Dreyer, K. Hamilton, P. F. Monni, and G. P. Salam, Logarithmic accuracy of parton showers: A fixed-order study, J. High Energy Phys. 09 (2018) 033; Erratum, J. High Energy Phys. 03 (2020) 083].

[15] G. Bewick, S. Ferrario Ravasio, P. Richardson, and M. H. Seymour, Logarithmic accuracy of angular-ordered parton showers, J. High Energy Phys. 04 (2020) 019.

[16] M. Dasgupta, F. A. Dreyer, K. Hamilton, P. F. Monni, G. P. Salam, and G. Soyez, Parton Showers beyond Leading Logarithmic Accuracy, Phys. Rev. Lett. 125, 052002 (2020).

[17] K. Hamilton, R. Medves, G. P. Salam, L. Scyboz, and G. Soyez, Colour and logarithmic accuracy in final-state parton showers, arXiv:2011.10054.

[18] Z. Nagy and D. E. Soper, What is a parton shower? Phys. Rev. D 98, 014034 (2018).

[19] Z. Nagy and D. E. Soper, Parton showers with quantum interference, J. High Energy Phys. 09 (2007) 114.

[20] Z. Nagy and D. E. Soper, Parton showers with quantum interference: leading color, spin averaged, J. High Energy Phys. 03 (2008) 030. 
[21] Z. Nagy and D. E. Soper, Parton showers with quantum interference: leading color, with spin, J. High Energy Phys. 07 (2008) 025.

[22] Z. Nagy and D. E. Soper, Parton shower evolution with subleading color, J. High Energy Phys. 06 (2012) 044.

[23] Z. Nagy and D. E. Soper, A parton shower based on factorization of the quantum density matrix, J. High Energy Phys. 06 (2014) 097.

[24] Z. Nagy and D. E. Soper, Ordering variable for parton showers, J. High Energy Phys. 06 (2014) 178.

[25] Z. Nagy and D. E. Soper, Summing threshold logs in a parton shower, J. High Energy Phys. 10 (2016) 019.

[26] Z. Nagy and D. E. Soper, Jets and threshold summation in DEDUCTOR, Phys. Rev. D 98, 014035 (2018).

[27] T. Sjöstrand et al., An introduction to PYTHIA 8.2, Comput. Phys. Commun. 191, 159 (2015).

[28] T. Gleisberg, S. Hoeche, F. Krauss, M. Schonherr, S. Schumann, F. Siegert, and J. Winter, Event generation with SHERPA 1.1, J. High Energy Phys. 02 (2009) 007.

[29] G. F. Sterman, Summation of large corrections to short distance hadronic cross-sections, Nucl. Phys. B281, 310 (1987).

[30] Z. Nagy and D. E. Soper, Evolution of parton showers and parton distribution functions, Phys. Rev. D 102, 014025 (2020).

[31] Z. Nagy and D. E. Soper, Parton showers with more exact color evolution, Phys. Rev. D 99, 054009 (2019).

[32] Z. Nagy and D. E. Soper, Exponentiating virtual imaginary contributions in a parton shower, Phys. Rev. D 100, 074005 (2019).
[33] S. Brandt, C. Peyrou, R. Sosnowski, and A. Wroblewski, The principal axis of jets-an attempt to analyse high-energy collisions as two-body processes, Phys. Lett. 12, 57 (1964).

[34] E. Farhi, Quantum Chromodynamics Test for Jets, Phys. Rev. Lett. 39, 1587 (1977).

[35] S. Catani, L. Trentadue, G. Turnock, and B. Webber, Resummation of large logarithms in $e^{+} e^{-}$event shape distributions, Nucl. Phys. B407, 3 (1993).

[36] D. E. Soper and L. R. Surguladze, On the QCD perturbative expansion for $e^{+} e^{-} \rightarrow$ hadrons, Phys. Rev. D 54, 4566 (1996).

[37] http://www.desy.de/ znagy/deductor/.

[38] http://pages.uoregon.edu/soper/deductor/.

[39] G. Gustafson, Multiplicity distributions in QCD cascades, Nucl. Phys. B392, 251 (1993).

[40] N. Fischer, S. Prestel, M. Ritzmann, and P. Skands, VINCIA for hadron colliders, Eur. Phys. J. C 76, 589 (2016).

[41] Z. Nagy and D. E. Soper, Summations of large logarithms by parton showers, arXiv:2011.04773v2.

[42] Z. Nagy and D. E. Soper, Summations by parton showers of large logarithms in electron-positron annihilation, arXiv: $2011.04777 \mathrm{v} 2$.

[43] J. Botts and G. F. Sterman, Hard elastic scattering in QCD: Leading behavior, Nucl. Phys. B325, 62 (1989).

[44] S. Catani and M. H. Seymour, A general algorithm for calculating jet cross-sections in NLO QCD, Nucl. Phys. B485, 291 (1997); Erratum, Nucl. Phys. 510, 503 (1998).

[45] T. Sjostrand and P.Z. Skands, Transverse-momentum-ordered showers and interleaved multiple interactions, Eur. Phys. J. C 39, 129 (2005). 